Plasticity of neurons in mouse major pelvic ganglia in response to loss of physiological input in cases of nerve injury and spinal cord injury

A Dissertation
Presented to
The Faculty of the Graduate School
At the University of Missouri
In Partial Fulfillment
Of the Requirements for the Degree
Doctor of Philosophy

By

Cindy Kyi

Dr. David Schulz, Dissertation Advisor

May 2018 
The undersigned, appointed by the dean of the Graduate School,

have examined the dissertation entitled

\title{
PLASTICITY OF NEURONS IN MOUSE MAJOR PELVIC GANGLIA IN RESPONSE TO LOSS OF PHYSIOLOGICAL INPUT IN CASES OF NERVE INJURY AND SPINAL CORD INJURY
}

\author{
Presented by Kyi, Cindy (MU-Student)
}

A candidate for the degree of

Doctor of Philosophy

And hereby certify that, in their opinion, it is worthy of acceptance.

Dr. David J. Schulz

Dr. Michael L. Garcia

Dr. Andrew D. McClellan

Dr. Kevin J. Cummings 


\section{Dedicated}

to

Mom and Dad 


\section{ACKNOWLEDGEMENTS}

First of all, I would like to thank God for guiding me and being with me and my family since the day I left Myanmar (Burma). He has been my rock and shelter through all the challenges along the way.

I am very grateful to my advisor Dr. David Schulz for granting me with the opportunity to work in his laboratory and believing in me to take on a new project. Thank you very much for all your guidance, patience, training, and for giving me room to grow under your mentorship.

I would also like to thank my committee members Dr. Andrew McClellan, Dr. Michael Garcia, and Dr. Kevin Cummings for all of their support in providing me with feedback not only on the details of experimental protocols but also helping me relate my experimental findings to a big picture context.

I would like to thank Dr. McClellan for always being there whenever I needed help with technical issues at any time of a day, and always providing with advices to better my experiments. I would like to thank Dr. Garcia for contributing his expertise in mouse models and on surgical protocols. I would like to thank Dr. Cummings for contributing his knowledge on autonomic nervous system.

I would also like to thank my labmates Kawasi Lett, Michael Gray, Brian Lane, Daniel Kick, Adam Northcutt, Joe Santin, Sherryl Henderson, and Jasmine Hall for providing me with a great intellectual environment and constant feedback on my work throughout my time in Schulz lab. Thank you, Virginia (Ginny) Garcia, for always being there and providing support in both professional and personal matters. 
I would like to thank the labs of Drs. Milescu, Dr.McClellan, Dr.Zars, and everyone from the neurobiology seminar group for all of their constructive feedback on my work.

I would like to thank the faculty and staff members from Division of Biological Sciences, University of Missouri, for providing support throughout my career as a graduate student.

Last, but not least, I would like to thank my family; dad, mom, and Leo, and my friends from near and far for their unwavering love and support throughout this journey. 


\section{TABLE OF CONTENTS}

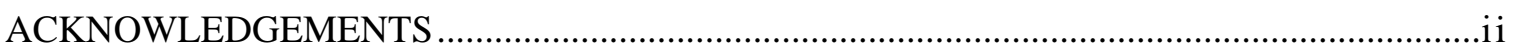

TABLE OF CONTENTS .....................................................................................................

LIST OF FIGURES ..........................................................................................................

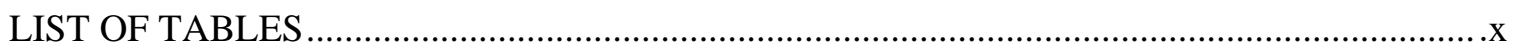

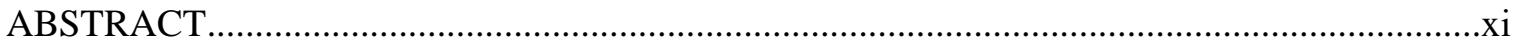

CHAPTER 1 INTRODUCTION .............................................................................. 1

$1.1 \quad$ Autonomic control of organ functions .................................................................

$1.2 \quad$ Neural control of lower urinary tract function ............................................................. 6

1.3 Anatomy and Physiology of autonomic pathways involved in control of micturition ....9

1.4 Morphological and physiological properties of major pelvic ganglia............................10

1.5 Cholinergic neurotransmitter system and its role in autonomic functions .....................12

1.5.1 Ion permeablities of nAChR subunits and their roles in synaptic transmission......14

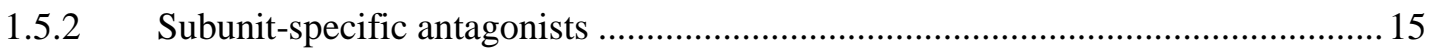

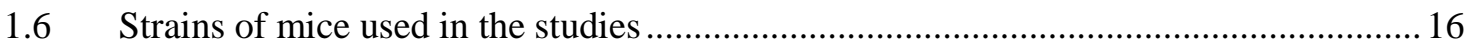

CHAPTER 2 CHARACTERIZATION OF SYNAPTIC TRANSMISSION AT THE NEURONS

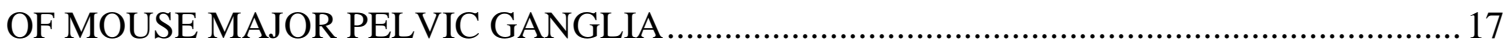

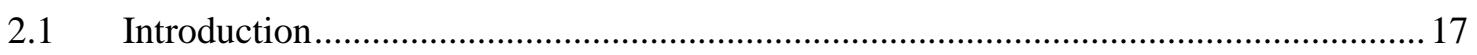

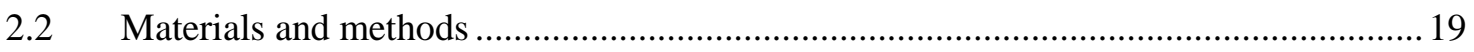

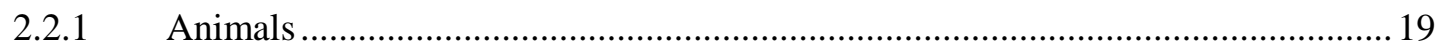

2.2.2 Tissue isolation for quantitative PCR (qPCR) ……………………………..... 19

2.2.3 Quantitative PCR ...................................................................................... 20

2.2.4 Tissue isolation for in-vitro recordings .........................................................2 22

2.2.5 In-vitro electrophysiology recordings ..............................................................22

2.2.6 Tests for membrane and action potential properties .............................................23

2.2.7 Nerve stimulation and synaptic properties test .................................................23

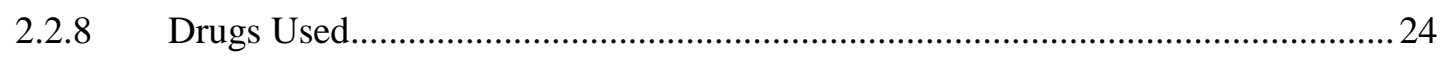

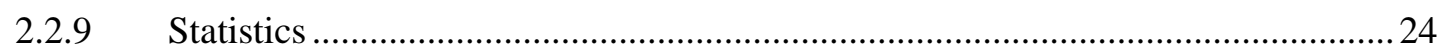




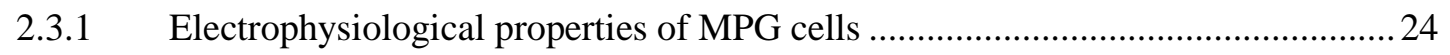

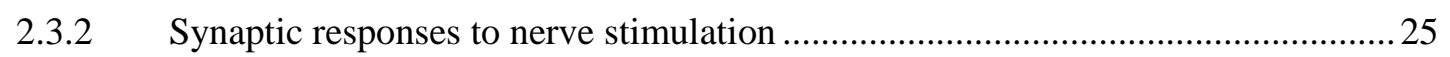

2.3.3 Membrane properties of neurons in major pelvic ganglia.....................................26

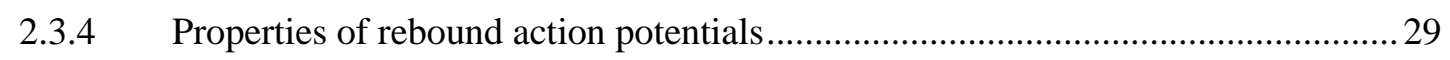

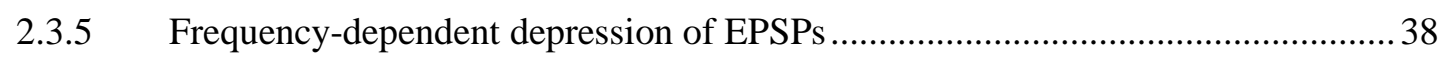

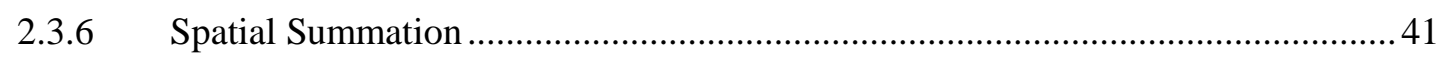

2.3.7 Nicotinic acetylcholine receptor subtypes involved in synaptic transmission ........42

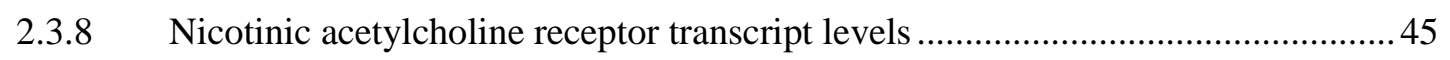

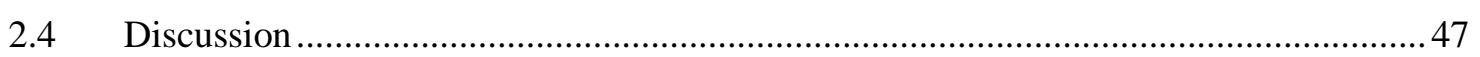

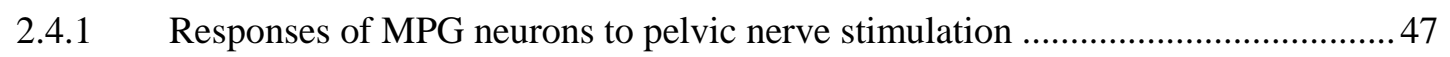

2.4.2 Membrane properties and action potential properties of MPG neurons ................. 48

2.4.3 Frequency-dependent modulation of synaptic transmission at the MPG neurons .50

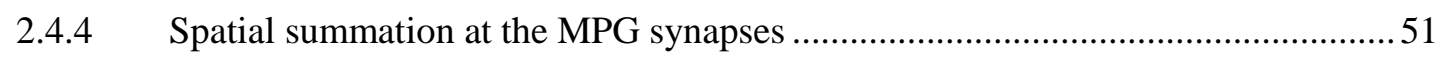

2.4.5 mRNA expression of nicotinic acetylcholine receptor (nAChR) subunits ............51

CHAPTER 3 EFFECTS OF DECENTRALIZATION ON CHOLINERGIC NEUROTRANSMISSION AT THE NEURONS OF MOUSE MAJOR PELVIC GANGLIA.... 55

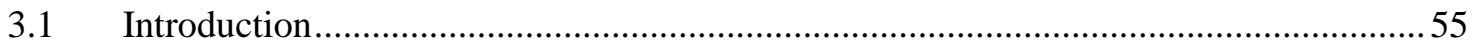

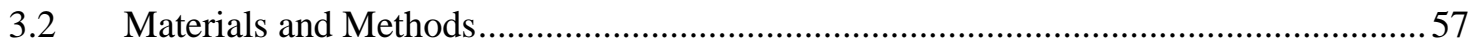

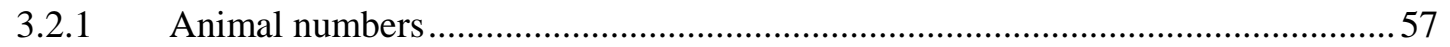

3.2.2 Preparing the animals and induction of anesthesia .............................................5

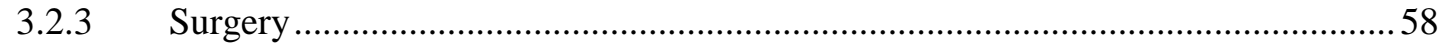

3.2.4 Tissue isolation for in-vitro recordings ................................................................. 59

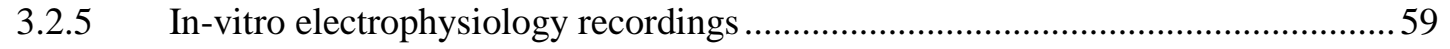

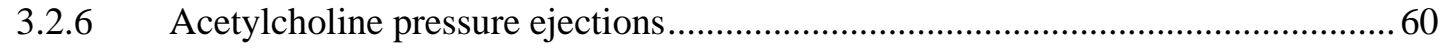

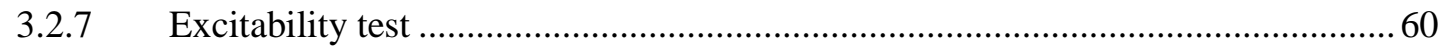

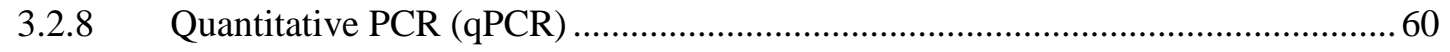

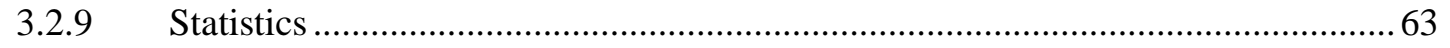

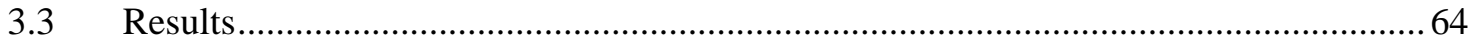

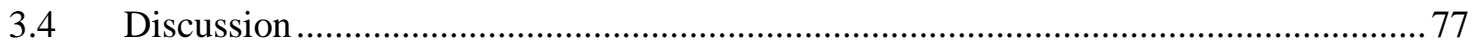

CHAPTER 4 EFFECTS OF SPINAL CORD INJURY ON SYNAPTIC, PASSIVE, AND ACTIVE PROPERTIES OF NEURONS OF MOUSE MAJOR PELVIC GANGLIA..................85

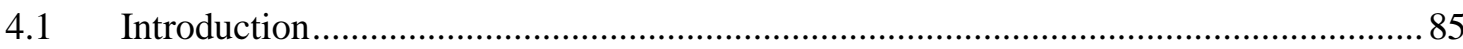




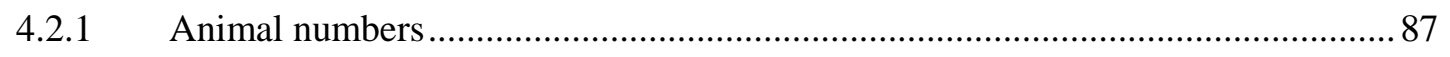

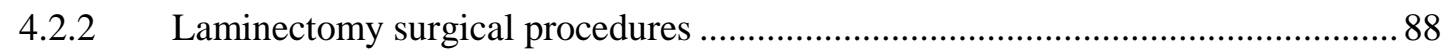

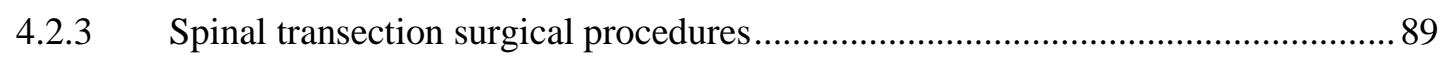

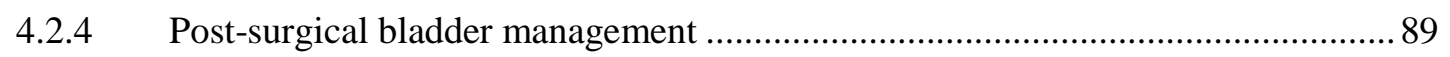

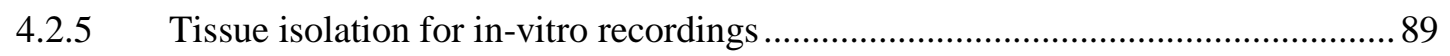

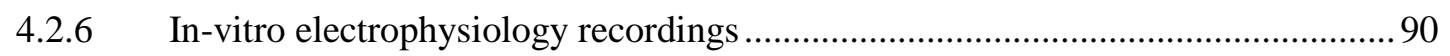

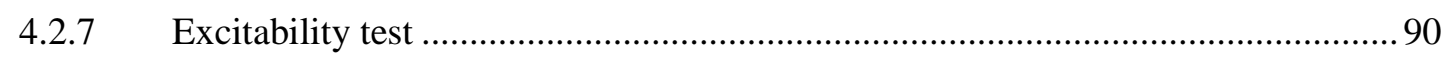

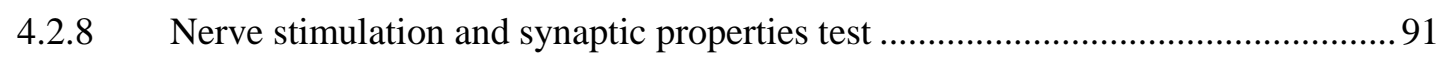

4.2.9 Acetylcholine pressure ejections...................................................................... 91

4.2.10 Real-time quantitative polymerase chain reactions (qPCR) ...............................92

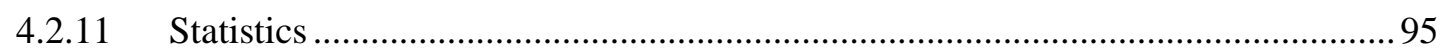

4.2.12 Changes in mRNA expressions of nAChRs and voltage-gated ion channels ....... 102

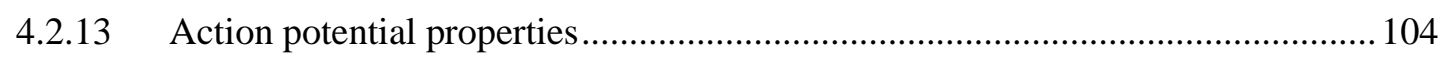

4.2.14 Response of the MPG neurons to pelvic nerve stimulation after SCI.................. 110

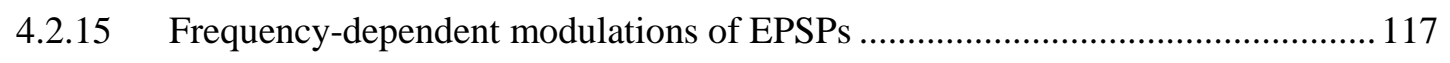

4.2.16 Frequency-dependent modulation of postsynaptic currents ................................. 118

4.3 Reliability of evoked action potentials at multiple frequencies .................................. 119

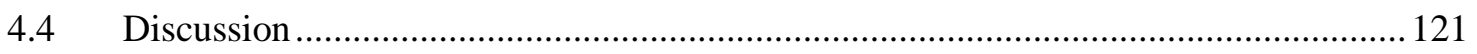

4.4.1 Cholinergic transmission at MPG neurons in acute and chronic SCI .................. 122

4.4.2 Excitability changes of MPG neurons in acute and chronic SCI ......................... 126

4.4.3 Changes in action potential properties of MPG neurons in acute and chronic SCI 128

4.4.4 Nerve stimulation experiments on the nature of preganglionic inputs to MPG after SCI 130

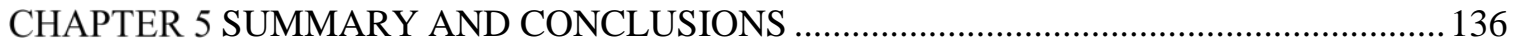

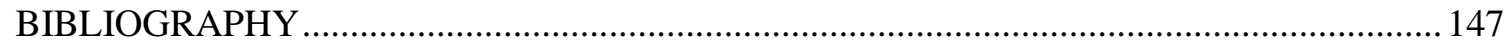

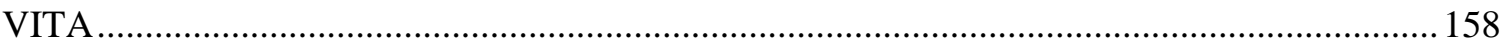




\section{LIST OF FIGURES}

Figure 1.1 Autonomic nervous system control of organ functions................................ 5

Figure 1.2 Neural control of micturition.................................................................. 8

Figure 1.3 Anatomy of neural pathways controlling micturition function. .................... 10

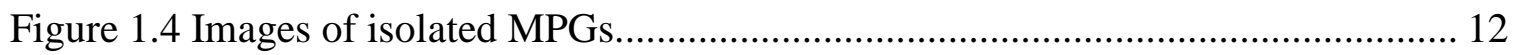

Figure 1.5 Illustration of molecular structure of nicotinic acetylcholine receptor (nAChR)

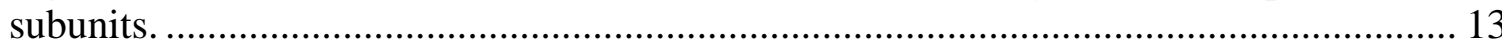

Figure 2.1 Voltage responses of MPG neurons to intracellular current injections .......... 25

Figure 2.2 Relationship between resting membrane potentials and types of responses to nerve stimulation.

Figure 2.3 Significant differences between properties of rebound action potentials were observed (see table 2.3) from action potential and EPSP generating neurons.

Figure 2.4 Representative traces showing various shapes and sizes of fast synaptic responses in response to pelvic nerve stimulation.

Figure 2.5 Frequency-dependent synaptic depression was observed at higher frequencies of pelvic nerve stimulation

Figure 2.6 Decreased in reliability of action potential firing was observed at higher frequencies of stimulation

Figure 2.7 Synaptic recruitment was observed in MPG neurons in response to pelvic nerve stimulation at increasing stimulus intensities.

Figure 2.8 Blockage of excitatory postsynaptic potentials (EPSPs) by selective synaptic blockers.

Figure 2.9 Nicotinic acetylcholine receptor antagonists reduced action potentials into subthreshold EPSPs 
Figure 2.10 (A) Analysis of abundance of mRNA transcripts showed $\alpha 3$ expression to be most abundant among other subunits. (B) $\alpha 3$ and $\beta 4$ subunits showed correlated expression of their mRNA expressions from whole MPGs. 46

Figure 3.1 MPG neurons from decentralized ganglia show increased current amplitudes in response to acetylcholine pressure ejections.

Figure 3.2 Current densities from decentralized MPG neurons were higher than the corresponding sham groups, however, capacitances were not significantly different between groups.

Figure 3.3 MPG neurons from decentralized groups show higher amplitudes of voltage responses than the sham groups, however, statistical significance is observed only between ipsilateral sides of 28-day sham and decentralized groups.

Figure 3.4 Excitability of the MPG neurons do not seem to alter either in terms of number of tonic cells or rheobase currents.

Figure 3.5 Passive membrane properties of MPG neurons from both sham and decentralized groups on both sides show very little differences.

Figure 3.6 qPCR analyses showed changes in neuronal nicotinic acetylcholine receptor subunits.

Figure 3.7 mRNA expression levels for CHRNA3 and CHRNB4 genes were found to be positively correlated across all experimental groups

Figure 4.1 (A) Schematic showing the experimental set-up of ACh pressure ejection and intracellular recording electrode (a) Pressure-ejection electrode (b) Impaled cell (c) Intracellular recording electrode (B) ChAT (BAC)-eGFP labelled parasympathetic neurons of $\mathrm{M}$

Figure 4.2 Synaptic strength of males were affected by acute SCI as well as with time after SCI whereas that of SCI females were not different compared to laminectomy groups

Figure 4.3 Percentage of phasic cells decreased considerably in chronic SCI MPG 98

Figure 4.4 Intrinsic properties of MPG neurons in females did not show significant changes between laminectomy and spinal cord injury groups 
Figure 4.5 Significant changes in expressions levels of mRNA transcripts for nicotinic receptor ( $\mathrm{nAChR}$ ) subunits and voltage-gated calcium, potassium and sodium channels from RT-qPCR analyses

Figure 4.6 Significant changes in action potential properties associated with narrower action potentials were observed in males at chronic SCI.

Figure 4.7 Significant changes in action potential properties associated with narrower action potentials were observed at acute SCI and with time post-injury in females. ..... 108

Figure 4.8 Depression of EPSPs were observed at higher frequencies of stimulations in both laminectomy groups and 3-day SCI, however, 1-month SCI group did not show

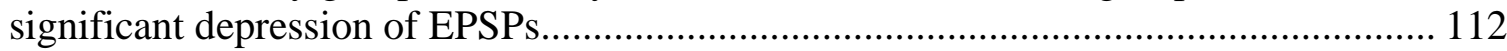

Figure 4.9 . Bar graphs showing percentage of action potentials fired in response to pelvic nerve stimulation at different frequencies. 


\section{LIST OF TABLES}

Table 2.1 Gene identification and primer sets used for real-time PCR reactions............ 22

Table 2.2 Membrane properties of the neurons in mouse major pelvic ganglia.............. 28

Table 2.3 Properties of rebound action potentials. ................................................. 31

Table 2.4 Properties of nerve-stimulated action potentials ...................................... 36

Table 2.5 Properties of nerve-stimulated EPSPs ................................................ 37

Table 3.1 Gene identification and primer sets used for real-time PCR reactions............. 63

Table 3.2 and Table 3.3 Electrical properties of MPG neurons from sham groups compared to corresponding decentralized groups .................................................. 73

Table 4.1 Gene identification and primer sets used for realtime PCR reactions ............. 95

Table 4.2 Table showing P-values for comparison of mRNA transcripts between acute laminectomy and acute SCI, and chronic laminectomy and chronic SCI groups .......... 102 


\begin{abstract}
Autonomic dysfunctions present significant effects on day to day functions of spinal cord-injured patients. Most people with spinal cord injury (SCI) reported a desire to recover autonomic functions such as bladder and sexual functions over regaining of locomotor functions. Lower urinary tract function is one of the autonomic functions that is impaired after SCI. Acute SCI results in areflexic bladder and complete urinary retention while patients with chronic SCI suffer from hyperreflexic bladder, incontinence and inefficient voiding. While extensive SCI research has been done locomotor recovery, relatively limited amount of research has been done on the underlying mechanisms of autonomic dysfunctions associated with SCI.
\end{abstract}

The major pelvic ganglia (MPG) are peripheral ganglia that consist of postganglionic neurons that innervate the urogenital organs and are a part of the neural pathway that controls micturition. In mice, there is one MPG on each side of the animal. MPG receives cholinergic parasympathetic input from the preganglionic neurons in the sacral cord through pelvic nerve and sympathetic cholinergic inputs from those in the lumbosacral cord through the hypogastric nerve. Nicotinic cholinergic transmitter system is the major system involved in ganglionic synaptic transmission at the MPG neurons.

The goal of this thesis is to understand the effects of neural injury on nicotinic cholinergic transmission at the postganglionic MPG neurons that innervate the urogenital organs. We are interested in the properties of MPG neurons in these injury states because these neurons are the final neurons in the autonomic pathway that directly innervate the target organs whose functions are compromised as results of these injuries. 
Before we could study the state of MPG neurons in injury conditions, we first needed to characterize the properties of these neurons in uninjured physiological state. By characterizing the normal cholinergic transmission in control mice, we are able to investigate the changes in the system after injury. Secondly, we sought to understand how MPG neurons respond to abrupt loss of all synaptic inputs. In these studies, we severed all the preganglionic neurons to MPG on one side of the animals leaving the other side intact. Finally, we studied the effects spinal cord injury on the synaptic transmission at the MPG. Spinal cord injury presumably presents altered forms of presynaptic inputs from the spinal cord to the MPG neurons due to hyperreflexic nature of the reflex pathway after SCI. We utilized molecular, electrophysiological, and pharmacological approaches using mouse models to answer our questions.

In the first chapter of the thesis, I characterized the synaptic, passive, and firing properties of the MPG neurons in both male and female mice. I also characterized the nicotinic acetylcholine receptor subunits involved in cholinergic neurotransmission at the MPG.

In the second chapter, I studied the effects of loss of direct inputs to the MPG neurons in both ipsilateral and contralateral intact ganglia in male mice. I performed unilateral decentralization of inputs to the MPG by severing pelvic and hypogastric nerves.

In the third chapter, I studied the effects of spinal cord injury on properties of presynaptic inputs to the MPG as well as postganglionic properties, passive properties and firing properties of MPG neurons. In this injury model, I performed complete transections of 
the spinal cords between thoracic spinal segments (T10 and T11) in both male and female mice. All the mice had impaired bladder reflexes after spinal transection.

Our results showed that decentralization and spinal cord injury effect the synaptic transmission at the MPG as well as the properties of the MPG neurons differently. These effects could be due to influences from both the nature of presynaptic input and the functional state of the target organ. We also observed different effects of spinal cord injury between MPG neurons of males and female. Understanding the mechanisms of changes at the neurotransmission at MPG neurons would be important in developing therapeutic measures for autonomic dysfunctions of the urogenital organs in nerve injury or in spinal cord injury. 


\section{CHAPTER 1 INTRODUCTION}

Lower urinary tract function (micturition function) is one of the autonomic functions that is impaired after spinal cord injury (SCI) among other autonomic disorders such as cardiac dysrhythmias, autonomic dysreflexia, sexual and bowel dysfunctions. A large amount of literature has been focused on the hyperactive sensory afferent mechanisms as the main cause driving hyperactive reflexes controlling the micturition functions. There is a significant lack of knowledge on how the efferent mechanisms in the lower urinary tract reflex pathways are affected by spinal cord injury.

It is important to understand the properties of efferent pathways and neurons after spinal cord injury because in addition to directly innervating the target organs, they have been proposed to play a role in modulation of peripheral sensory-motor feedback system (Persyn et al., 2016). Hence, our studies will focus on the properties of peripheral efferent neurons in the major pelvic ganglia (MPG) that innervate the urogenital organs and how they are affected by spinal cord injury.

Our lab is ultimately interested in the impairment of bladder function after spinal cord injury. However, the studies carried out in my thesis will serve as initial examination of all the parasympathetic neurons in the MPG to investigate whether they are affected by spinal cord injury. Prior to studying the effects of spinal cord injury, we studied the effects of decentralization of preganglionic inputs on the MPG neurons. Effects of decentralization or complete loss of preganglionic inputs to MPG neurons will serve as a 
point of comparison to altered hyper-reflexic inputs in spinal cord injury. In this introduction, I will provide the background information on autonomic control of organ functions, neural control of lower urinary tract function, morphological and physiological properties of major pelvic ganglia, cholinergic neurotransmitter system and its role in autonomic functions, and different strains of mice used in our studies.

\subsection{Autonomic control of organ functions}

Many of the organ functions are regulated by the autonomic division of the peripheral nervous system. Autonomic nervous system consists of preganglionic neurons residing in the spinal cord which send out efferent spinal outputs to the target organs after making synaptic contacts in various peripheral ganglia. The preganglionic autonomic neurons receive sensory inputs originating from the target organs through afferent axons traveling in autonomic pathways through cranial and spinal nerves (Kuntz, 1936).

Human spinal cord is divided into 8 cervical, 12 thoracic, 5 lumbar, 5 sacral, and 3 coccygeal segments (Fowler et al., 2008). In mice, spinal segments are divided into 8 cervical, 13 thoracic, 6 lumbar, 4 sacral and 3 coccygeal segments (Fowler et al., 2008).

In the sympathetic division of autonomic nervous system, the efferent nerves exiting the thoracolumbar segments (T1-L2) of the spinal cord make synaptic contacts with corresponding ganglia in a chain of paravertebral ganglia called sympathetic chain ganglia (Mccorry, 2007). After exiting the sympathetic chain ganglia, sympathetic nerves at the thoraco-lumbar levels innervate the target organs (Gray, 1918) (Fig. 1.1). Splanchnic nerves from the lower thoracic segments that innervate the digestive organs, liver, pancreas, adrenal gland, small intestine and large intestine make additional synaptic 
contacts in celiac ganglion and superior mesenteric ganglion (Gray, 1918) (Fig. 1.1). The sympathetic nerves from the lumbar segments that innervate large intestines, kidneys, bladder and the sex organs make additional synaptic contacts in the inferior mesenteric ganglion and major pelvic ganglion before innervating the target organs (Gray, 1918)

(Fig.1.1). The celiac ganglia, superior and inferior mesenteric ganglia in the sympathetic pathways are also called prevertebral ganglia (Gray, 1918) (Fig.1.1).

In the parasympathetic division of the autonomic nervous system, sensory afferent information from thoracic and abdominal viscera is carried in the cranial nerves and the vagus nerve and conveyed to the brainstem (Kuntz, 1936; McCorry, 2007). The cranial nerves (III, VII, IX, and X) make synaptic contacts in prevertebral ganglia such as ciliary ganglion, pterygopalatine ganglion, submandibular ganglion, and otic ganglion. The parasympathetic nerves exiting the sacral cords (S2-S4) make synaptic contacts in major pelvic ganglia before innervating the distal colon and urogenital organs.

In sympathetic pathways, the major neurotransmitters involved in preganglionic neurotransmission is acetylcholine whereas the neurotransmitter for the postganglionic transmission is noradrenaline (McCorry, 2007). In parasympathetic pathways, the neurotransmitter involved in both preganglionic and postganglionic synaptic transmission is acetylcholine (McCorry, 2007). Nicotinic acetylcholine receptors are the major receptors involved in the synaptic transmission at the peripheral ganglia (Biasi., 2002).

The synaptic ratio between preganglionic neurons and postganglionic neurons are reported to be about 1:20 (McCorry, 2007) in sympathetic and 1:3 (McCorry, 2007) in parasympathetic pathways. Hence, there is a wide divergence of one preganglionic 
neuron innervating multiple postganglionic neurons in sympathetic prevertebral ganglia. The efferent limb of the autonomic activity is mostly controlled by reflex mechanisms (McCorry, 2007). Hence, the existence of a large number of postganglionic neurons could provide efficient reflex control of multiple target organs that the neurons in the prevertebral ganglia innervate (Kuntz,1936).

Sensory afferent nerve fibers also travel in autonomic nerves in opposite direction from those of the efferent nerves. The cell bodies of the afferents are located in dorsal root ganglia of corresponding spinal segments. They either make synapses directly onto the spinal preganglionic autonomic neurons or synapse on interneurons in the spinal cord to coordinate reflex mechanisms in regulation of organ functions. 


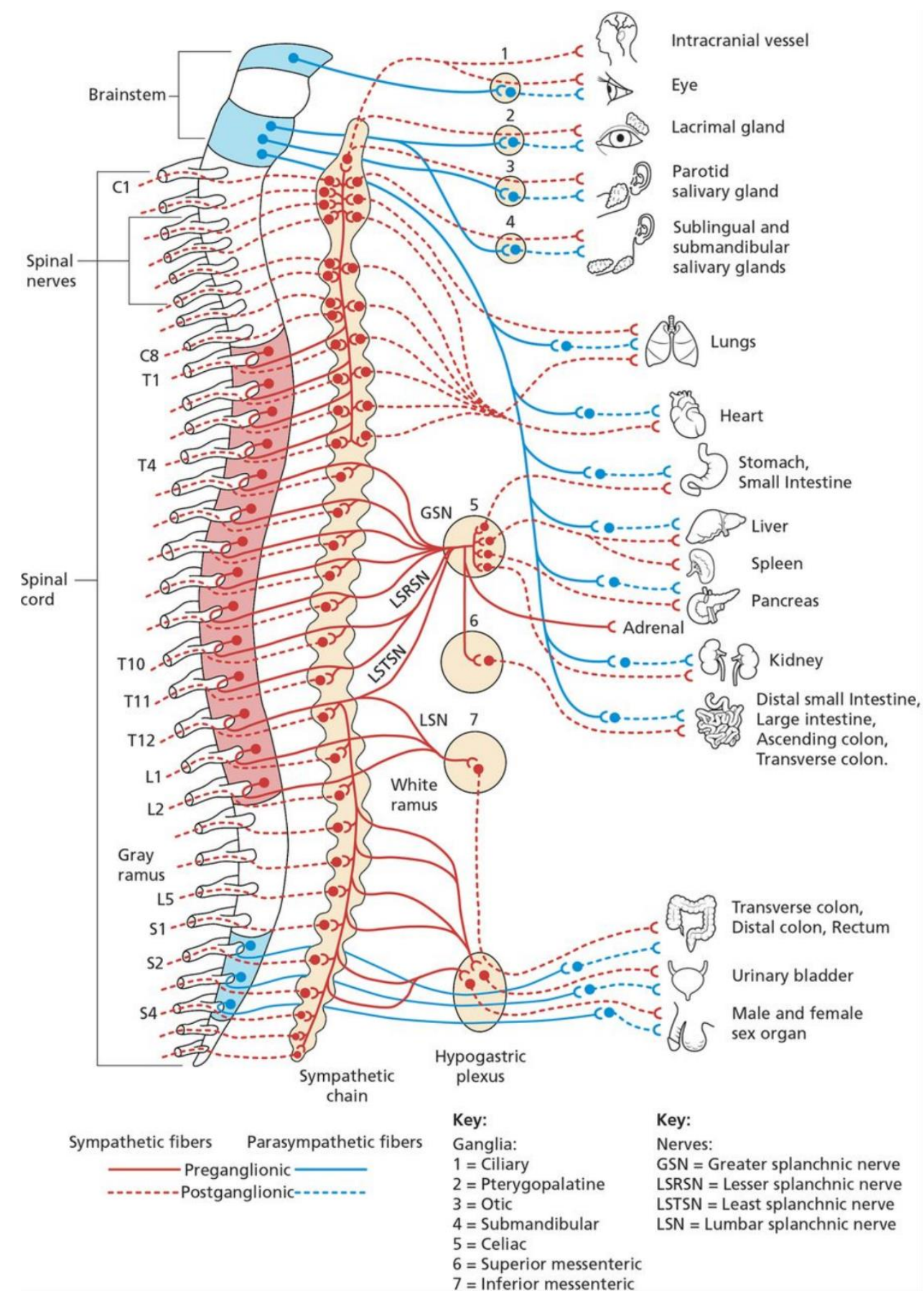

Figure 1.1 Autonomic nervous system control of organ functions.

Anatomy of autonomic nervous system, neural pathways, ganglia, organs and glands under autonomic control. Parasympathetic pathways are shown in blue and sympathetic pathways are show in red. (Bankenahally \& Krovvidi, 2016) The image of the diagram and associated information acquired from (Autonomic nervous system: anatomy, physiology, and relevance in anaesthesia and critical care medicine, BJA Education, 16 (11): 381-387 (2016) 


\subsection{Neural control of lower urinary tract function}

The two main functions of mature lower urinary tract are storage of urine during filling of bladder and elimination of urine when the bladder is full. Both processes are under the control of central and peripheral nervous systems (Figure1.1, 1.2, 1.3). The neural circuitry in the brain and spinal cord control the switching between storage and voluntary voiding functions at appropriate times(de Groat, 1995; Birder et al., 2010; de Groat and Yoshimura, 2015). The storage function of micturition is mostly under tonic inhibitory control of sympathetic nervous system and voiding function is mostly under the control of parasympathetic nervous system (de Groat, 1995; Birder et al., 2010; de Groat \& Yoshimura, 2015) (Figure1.2). During the storage phase, distension of the bladder sends low-level afferent signals to the pontine storage center (PSC) in the brainstem (de Groat, 1995; Birder et al., 2010; de Groat \& Yoshimura, 2015). This stimulates the sympathetic hypogastric nerve which innervates the bladder base and urethra, and pudental nerve which innervates the external urethral sphincter (EUS) (de Groat, 1995; Birder et al., 2010; de Groat \& Yoshimura, 2015) (Figure1). Activation of sympathetic nerve contracts bladder outlet and relaxes bladder detrusor, while activation of pudendal nerve contracts the EUS, retaining the urine in the bladder (Pastelin et al., 2012). PSC might also increase the activity of EUS (Pastelin et al., 2012). Hence, the sympathetic pathway represents spinal guarding reflexes which promotes continence (de Groat, 1995; Birder et al., 2010; de Groat \& Yoshimura, 2015). Bladder fullness causes intense firing of bladder afferent signals that travel up in parasympathetic pelvic nerve to the periaqueductal grey (PAG), a relay center in the brainstem (de Groat \&Yoshimura, 2015). The signals travel through the pontine micturition center (PMC) (Barrington's nucleus), where the switching signal 
from storage to voiding is thought to take place (de Groat \& Yoshimura, 2015). The mechanisms that underlie the switch and conscious bladder sensation are assumed to involve cerebral circuits above the PAG (de Groat, 1995; Birder et al., 2010; de Groat \& Yoshimura, 2015). This voiding reflex pathway stimulates parasympathetic pelvic nerve innervation of the bladder detrusor and inhibits the sympathetic innervation of urethra and pudendal innervation of EUS (de Groat \& Yoshimura, 2015). Parasympathetic innervation of the bladder causes the contraction of the detrusor, and inhibition of sympathetic and pudendal innervation which causes relaxation of the urethra and EUS, thereby enabling the bladder to void the urine (de Groat, 1995; Birder et al., 2010; Pastelin et al., 2012; de Groat \& Yoshimura, 2015). 


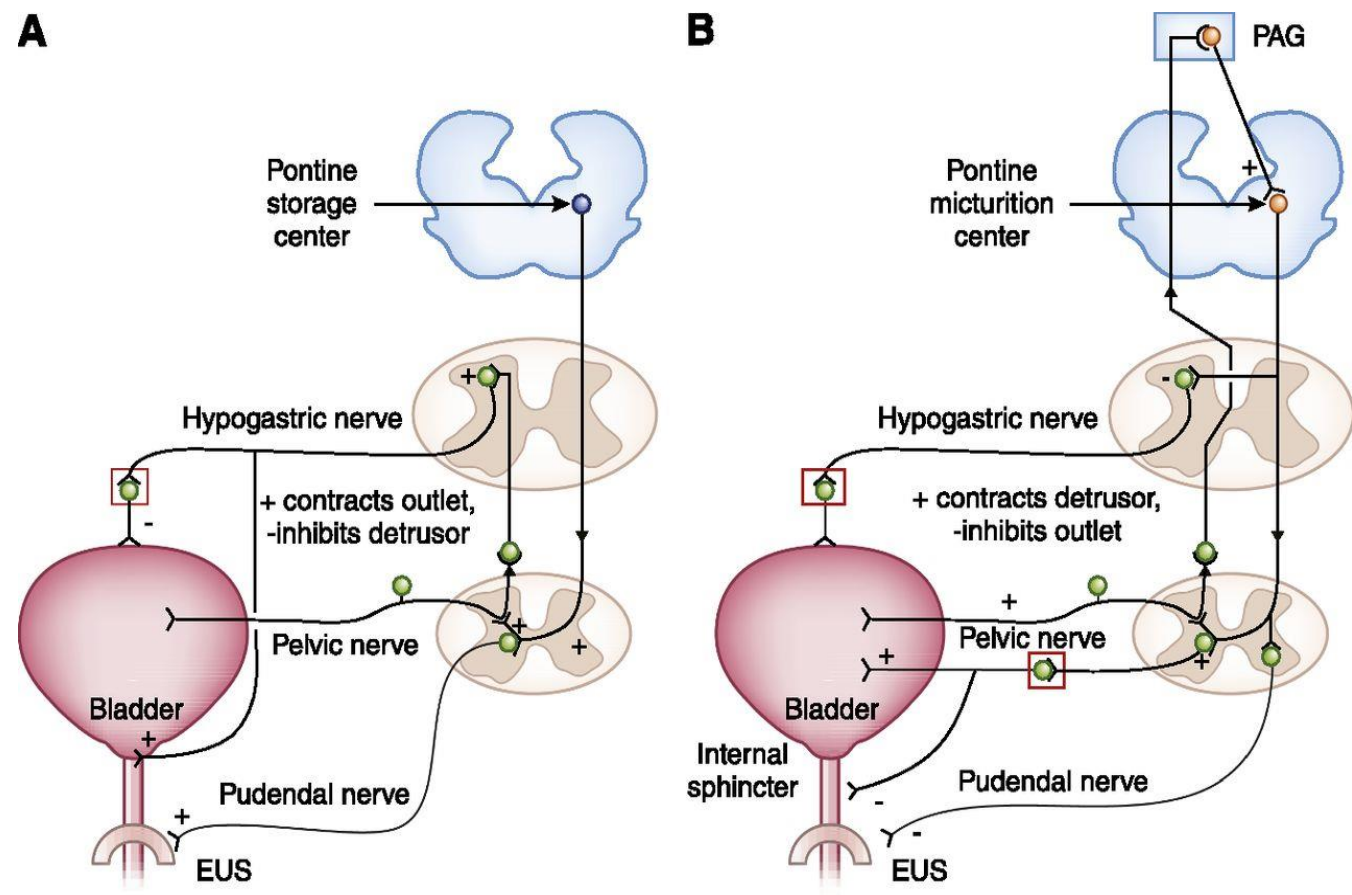

Figure 1.2 Neural control of micturition.

(A) Storage phase. When the bladder is being filled, low-level afferent activity through the pelvic nerve activates the sympathetic preganglionic neurons(SPN) which send the efferent signals through the hypogastric nerve to inhibit the detrusor and excite the bladder neck (internal sphincter). Inhibition of detrusor keeps the bladder relaxed while excitation of bladder neck keeps the bladder sealed during filling. The signals from pontine storage center in the brain stem also excite the somatic outflow to the external urethral sphincter (EUS)through pudendal nerve, keeping the EUS closed during bladder filling. (B) Voiding phase. When the intravesical pressure reaches the threshold for micturition, the afferent signals from the bladder wall are sent through the pelvic nerve to the periaqueductal gray area, and then to the pontine micturition center (PMC). The efferent signals from the PMC activate the parasympathetic preganglionic neurons which send efferent signals though the pelvic nerve to excite the bladder wall and inhibit the internal sphincter causing contraction of detrusor and relaxation of internal sphincter. The descending efferent signals inhibit the guarding reflex by inhibiting SPNs. The EUS is also relaxed by descending inhibition of Onuf's nucleus in the sacral cord, hence, allowing urine to be voided during the voiding phase. Figure and information adapted and modified from (Hill, 2015) from Clinical Journal of the American Society of Nephrology10: 480-492, 2015. doi: 10.2215/CJN.04520413. 


\subsection{Anatomy and Physiology of autonomic pathways involved in control of micturition}

Parasympathetic preganglionic neurons are located in the intermediolateral (IML) column of the spinal segments L6-S1 in rat and S2-S4 in human, and postganglionic neurons are located in pelvic ganglia and on bladder detrusor muscle (Berkley et al., 1993; Fowler et al., 2008; Inskip et al. 2009) (Figure 1.3). Acetylcholine (ACh) is the major neurotransmitter involved in synaptic transmission at both pre and post ganglionic synapses (de Groat \& Yoshimura, 2015). The sympathetic preganglionic neurons are located in the IML column of the spinal segments T10-L3 in rat and T11-L2 in human, and postganglionic neurons are located in inferior mesenteric ganglia (IMG), pelvic ganglia,and they innervate bladder detrusor muscle and bladder neck (Berkley et al., 1993; Fowler et.al., 2008; Inskip et.al.,2009) (Figure 1.3). The neurotransmitters involved in the sympathetic pathways are acetylcholine at the preganglionic synapses and noradrenaline (NA) at the postganglionic synapses (Langworthy, 1965; de Groat \& Yoshimura, 2015).

Parasympathetic afferents and efferents travel in pelvic nerve whereas sympathetic afferents and efferents travel in the hypogastric nerve (Fowler et. al., 2008). Somatic nerve supply to the EUS arises from motor neurons in S2-S4 spinal segments through the pudendal nerve (Pastelin et al., 2012; de Groat \&Yoshimura, 2015) (Figure 1.3). Somatic axons in the pudendal nerve also release ACh and cause contraction of the EUS (Fowler et. al., 2008). 


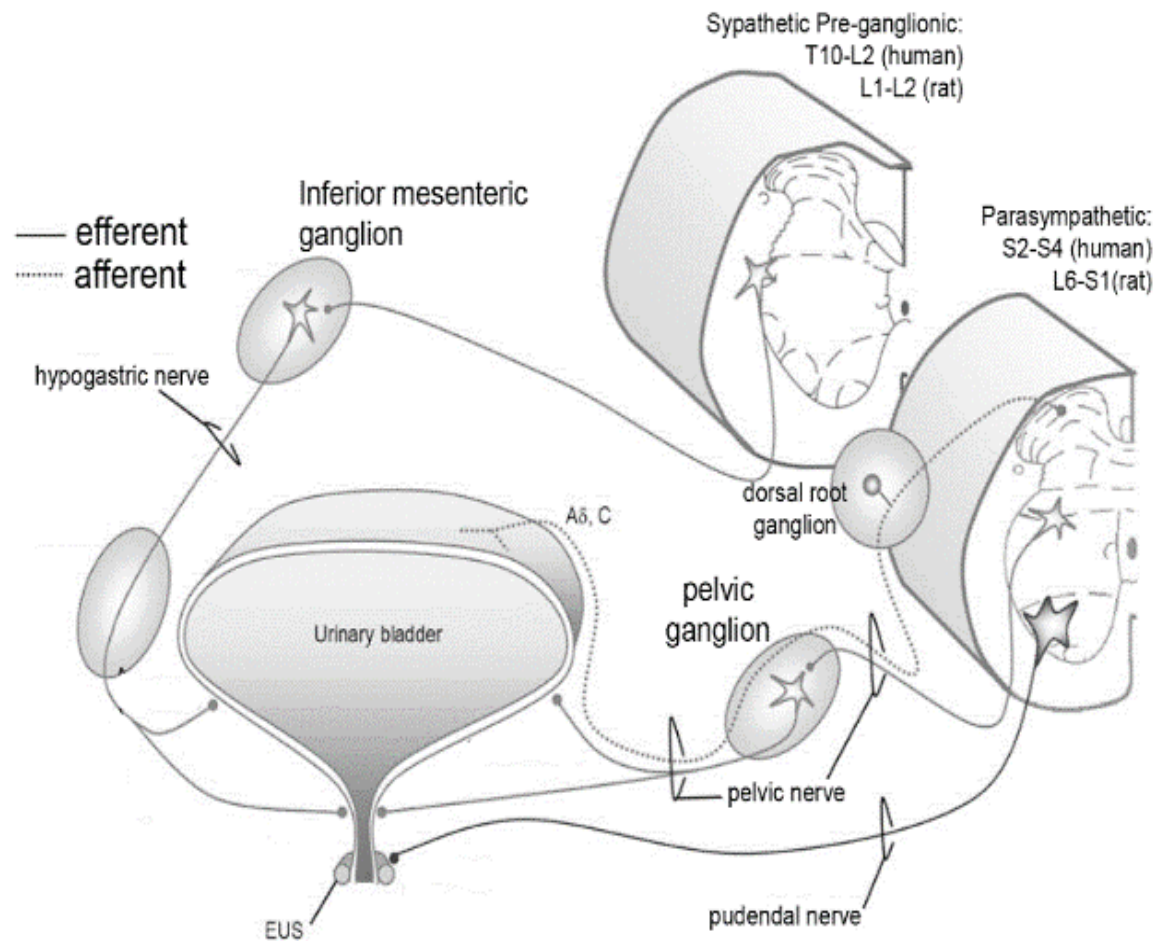

Figure 1.3 Anatomy of neural pathways controlling micturition function.

The schematic shows sympathetic and parasympathetic pathways originating from preganglionic neurons in the intermediolateral column of lumbar and sacral cords, respectively, and synapsing with postganglionic neurons in the inferior mesenteric and pelvic ganglia, before innervating the bladder. (Inskip et al., 2009) (Figure and information acquired from Autonomic assessment of animals with spinal cord injury: tools, techniques and translation. Spinal Cord (2009) 47, 2-35)

\subsection{Morphological and physiological properties of major pelvic ganglia}

Major pelvic ganglion (MPG) is a mixed ganglion containing sympathetic and

parasympathetic neurons innervating pelvic organs such as urinary bladder, colon and the genitals (Purinton, 1972; Keast, 1995). There is one MPG on each side of the bladder within the pelvic region. The major inputs to the MPG are pelvic nerve (parasympathetic) and hypogastric nerve (sympathetic). The major postganglionic nerves include bladder nerves that innervate the bladder and cavernous nerve that innervate the genitals (Keast, 1995; Girard et al., 2013). In figure 1.4, the left picture shows a dissected MPG from the right side of a male mouse with input nerve fibers attached. The picture on the right side of figure 3 is a female MPG labeled with microtubule-activated protein2 which stains 
neurons and Tyrosine Hydroxylase enzyme which marks sympathetic neurons in the ganglia (Jobling \& Lim, 2008).

Mouse MPG have comparable structural features as those of rats in terms of basic macroscopic organization and cell structure (Wanigasekara et al., 2003). The ganglia mostly contain cholinergic as well as adrenergic cells, and small intensely fluorescent (SIF) cells (Dail, 1996; de Groat \& Yoshimura, 2015). In both species, almost all the pelvic ganglion cells are monopolar, and either noradrenergic or cholinergic but not both (Wanigasekara et al., 2003). Rodent pelvic ganglia have no or very few short dendrites compared to higher mammalian species such as cat ganglia that have more complex morphology with 6-7dendrites (Dail, 1996; de Groat \&Yoshimura, 2015).

MPG receive cholinergic parasympathetic inputs from the preganglionic neurons in the sacral cord through pelvic nerve, and sympathetic cholinergic inputs from those in the lumbosacral cord through hypogastric nerve via inferior mesenteric ganglion (Kihara \& de Groat 1997; Keast 1995). The postganglionic axons from the pelvic ganglion synapse onto neurons on the bladder detrusor and urethral smooth muscles, releasing acetylcholine from parasympathetic axons and noradrenaline from sympathetic axons (Langworthy, 1965; Keast, 1995; de Groat \& Yoshimura, 2015). Majority of sympathetic neurons in the ganglia express tyrosine hydroxylase $(\mathrm{TH})$ and parasympathetic neurons express choline acetyltransferase(ChAT), the biosynthetic enzyme that converts choline precursor to acetylcholine (Tan et al., 2007). Synaptic transmission at the MPG neurons is mediated by acetylcholine acting on nicotinic receptors although other neurotransmitters could modulate the transmission (Dail et al., 1975; de Groat \& Saum 
1976; de Groat \& Yoshimura, 2015; Papka et al., 1999; de Groat \& Yoshimura, 2015).

Characterization of mouse and rat MPG showed that there are mainly two types of neurons- phasic and tonic spiking neurons according to their discharge characteristics in response to depolarizing current pulses (Tan et al., 2007; Jobling \& Lim, 2008).
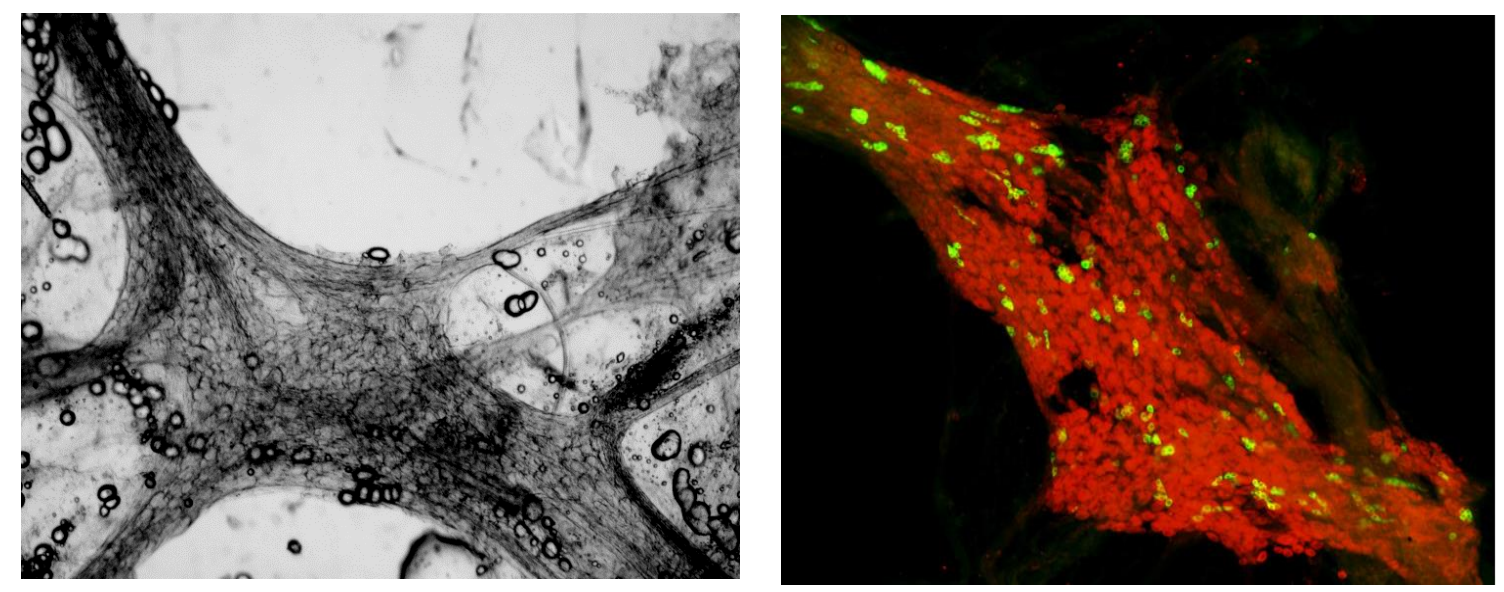

Figure 1.4 Images of isolated MPGs.

Whole male MPG (4x) (Left). Double-labeled neurons of female pelvic ganglion (Paracervical ganglion) with MAP2 (red) and TH (green) (Right). (Figure on the right side was acquired from Jobling \&Lim, 2008).

\subsection{Cholinergic neurotransmitter system and its role in autonomic functions}

Neuronal nicotinic acetylcholine receptors (nAChRs) play a key role in the control of bladder function as mediators of both parasympathetic and sympathetic fast synaptic transmission at MPG (de Groat \& Saum, 1976; Gallagher et al., 1982; De Biasi, 2002; Park et al., 2006 ). The nature of subunits involved in neurotransmission at MPG has not been fully characterized.

nAChRs are ligand-gated ion channels composed of five subunits to form a homopentamer or heteropentamer (Figure 1.5) (Albuquerque et al., 2009; Park et al., 2006; Skok, 2002). Twelve genes have been identified to encode neuronal nAChR 
subunits: $\alpha(2-10)$ and $\beta(2-4)$ subunits (De Biasi, 2002). The main subunits found in autonomic ganglia are $\alpha 3, \alpha 4, \alpha 5, \alpha 7, \beta 3$, and $\beta 4$ (Skok, 2002). $\alpha 2, \alpha 3$, and $\alpha 4$ subunits can form functional channels only if co-expressed with a $\beta 2$ or $\beta 4$ subunit (Skok, 2002;

Par
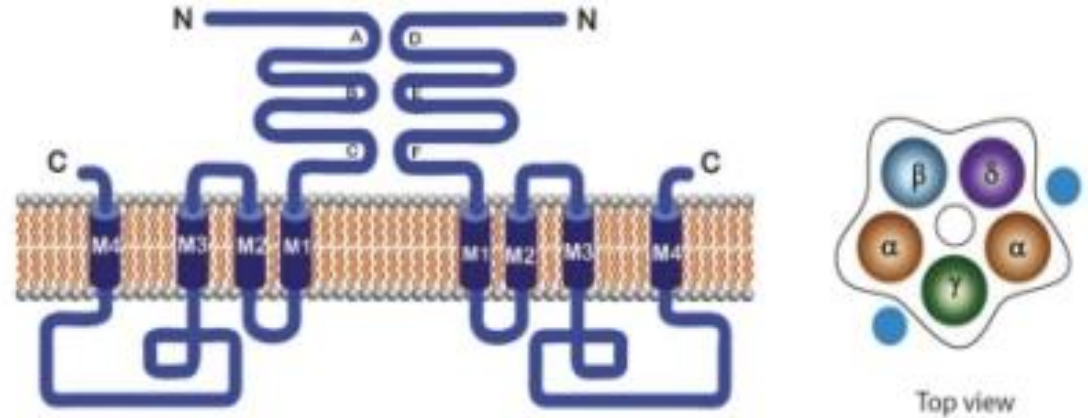

Top view

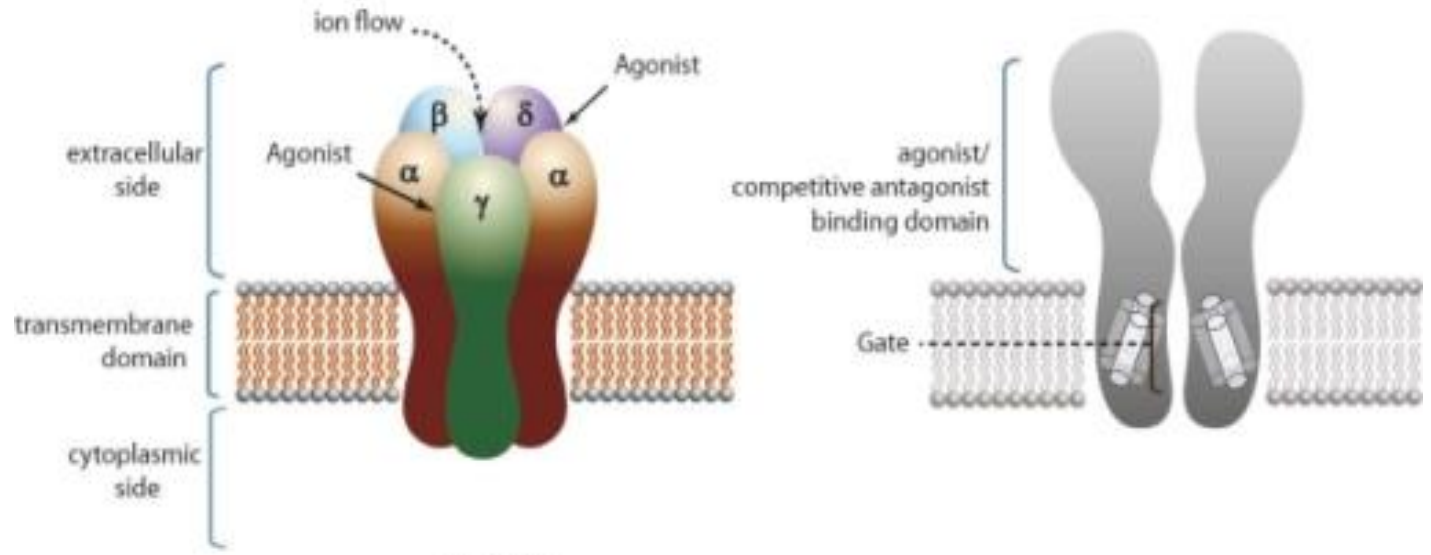

Side view

Figure 1.5 Illustration of molecular structure of nicotinic acetylcholine receptor (nAChR) subunits.

Top left picture shows four domains M1-M4 on a subunit spanning cellular membrane. Top right picture shows an example of a $\mathrm{nAChR}$ heteropentamer. Bottom left picture shows a nAChR subunit with channel pore for ion flow and agonist binding sites on $\alpha$ subunits. Bottom right picture shows agonist/competitive antagonist binding domain of a subunit across cell membrane. (Picture and information acquired from (Nasiripourdori et al., 2011) and www.columbia.edu, Arthur Karlin)

Generally, the subunits assemble in the $2 \alpha, 3 \beta$ stoichiometry (Skok, 2002). Each subunit has four transmembrane segments M1-M4 (Figure 1.5). Each $\alpha$ subunit has a receptor site that, when occupied by a nicotinic agonist, causes a conformational change in the protein (channel opening) that allows sodium and potassium ions to diffuse rapidly down their 
concentration gradients (Wang et al., 2002; Katzung et al., 2012). Differences in the structures of the channel proteins may produce differences in ion permeabilities which leads to differences in choline transmission (Wang et al., 2002; Dajas-Bailador \& Wonnacott, 2004). The primary effect of nicotinic receptor activation is depolarization of the nerve cell or neuromuscular end plate membrane via influx of $\mathrm{Na}^{+}$and $\mathrm{K}^{+}$with contributions from $\mathrm{Ca}^{2+}$ through the receptor channel (Katzung et.al, 2012). Some nAChR subtype signaling also seems to have long-term effects on metabolic pathways and gene expression via influx of calcium (Wang et al., 2002; Dajas-Bailador \& Wonnacott, 2004). The influx of $\mathrm{Ca}^{2+}$ into the neuron upon $\mathrm{AChR}$ activation, either through the nAChRs or through the opening of voltage-gated calcium channels, stimulates $\mathrm{Ca}^{2+-}$ dependent kinases such as protein kinase $\mathrm{C}$ and MAPK/ MEK kinases which can regulate cell signaling and gene expressions (Albuquerque et al.,1996; Wang et al., 2002; Dajas-Bailador \& Wonnacott, 2004; Jensen.et.al, 2005).

\subsubsection{Ion permeablities of nAChR subunits and their roles in synaptic transmission}

$\alpha 3$ subunit is found to be a predominant subunit in the autonomic ganglia and thought to form heteromultimeric nAChRs with other $\alpha$ and $\beta$ subunits (Skok, 2002; Wang et al., 2002). The subunit mediates the monovalent cation and $\mathrm{Ca}^{2+}$ conductance increases in response to ACh and nicotinic agonists (Skok, 2002). Most frequently, $\alpha 3 \beta 4$ combination is referred to as the "ganglia-type" nAChR (Skok, 2002; Wang et al., 2002). The $\alpha 5$ subunit is considered to be structural than ACh binding and is always co-assembled with $\alpha 3$ subunit in chick ciliary ganglion (Skok, 2002; Wang et al., 2002; Wonnacott \& Barik, 2007). According to studies in Xenopus oocytes, the presence of $\alpha 5$ subunit alters desensitization, pharmacology and $\mathrm{Ca}^{2+}$ permeability of the nAChRs containing $\alpha 3$ and 
$\beta 2$, or $\alpha 3$ and $\beta 4$ subunits (Skok, 2002; Wang et al.,2002). $\alpha 7 \mathrm{nAChRs}$ can form functional homomers of pentameric structure (Skok, 2002). Their rapid desensitization, high permeability for $\mathrm{Ca}^{2+}$ allows them to influence a variety of $\mathrm{Ca}^{2+}$ dependent events in autonomic neurons (Skok, 2002; Wang et al., 2002). The nature of $\beta$ subunits is the major factor in determining agonist affinity ((Parker et al., 1998; Wonnacott \& Barik, 2007). Differences in structures of the channel proteins formed by different neuronal nAChR phenotypes will have different ion permeabilities and conductance which result in different physiological function in cholinergic transmissions (Felix et al., 1998; Wang et al., 2002).

\subsubsection{Subunit-specific antagonists}

Nicotinic receptors in ganglia are blocked competitively by trimethaphan and noncompetitively by hexamethonium and are resistant to snake $\alpha$-toxins (Wonnacott $\&$ Barik, 2007). Ganglionic blocking agent tetraethylammonium blocks nAChR via both competitive and open-channel blocking mechanisms (Wonnacott \& Barik, 2007). Dsparteine, another ganglionic blocking agent blocks through competitive mechanisms (Wonnacott $\&$ Barik, 2007). Blockade of rat $\alpha 3 \beta 2$ and $\alpha 3 \beta 4$ nAChRs by hexamthonium, mecamylamine, petolinium and trimetaphan is determined by $\beta$ subunits (Wonnacott \& Barik, 2007). $\alpha 3 \beta 4$ subunit combination is selectively sensitive to blocking effect of dextromethorphan and its metabolite dextrorphan (Wonnacott \& Barik, 2007). $\alpha$ Conotoxin MII blocks $\beta 3$ containing $\mathrm{nAChRs}$ and is sensitive to subpopulations of $\alpha 3 \beta 2 \beta 3$ containing receptor. There are conotoxins specific for the $\alpha 7, \alpha 3, \beta 2$ and $\alpha 3 \beta 4$ nicotinic receptor subtypes (Wonnacott \& Barik, 2007). The $\alpha$-conotoxin AuIB selectively blocks $\alpha 3 \beta 4$ (ganglion-type) nAChRs (Wonnacott \& Barik, 2007). The MLA 
(methyllycaconitine) and $\alpha$-IMI conotoxin are extremely potent selective antagonists while choline and 1, 1-dimethy4-phenylpiperazine (DMPP) are selective agonists for $\alpha 7$ nAChRs (Wonnacott \& Barik, 2007).

\subsection{Strains of mice used in the studies}

We used Swiss Webster (CFW) outbred mice from Charles River Laboratory in Chapter 1 and 2.

In Chapter (3), we used transgenic mouse strain B6. Cg-Tg (RP23-268L19-EGFP)2 Mik/J also known as ChAT ${ }^{\mathrm{BAC}}$ - eGFP from the Jackson Laboratory. According to the information provided from the Jackson Laboratory, in these mice, bacterial artificial chromosome (BAC) clone 268L19 from the RP 23 mouse genomic library was modified to insert an enhanced green fluorescent protein (EGFP) gene with polyA sequence at the initiation codon in ChAT exon 3(Mouse data sheet - 007902, The Jackson Library). The cloned BAC (ChAT BAC - eGFP) was then microinjected into the pronucleus of B6D2F2 fertilized mouse eggs (Mouse data sheet - 007902, The Jackson Library). These transgenic mice were bred to $\mathrm{C} 57 \mathrm{BL} / 6 \mathrm{~J}$ mice and transgenic offsprings were produced (Mouse data sheet - 007902, The Jackson Library).

These transgenic mice allowed us to visualize the parasympathetic neurons in the major pelvic ganglia under florescent filters with wavelengths of $440 \mathrm{~nm}$ to $470 \mathrm{~nm}$. Utilization of this transgenic line enabled us to selectively study the effects of spinal cord injury on cholinergic neurotransmission in the major pelvic ganglia. 


\section{CHAPTER 2 \\ CHARACTERIZATION OF SYNAPTIC TRANSMISSION AT THE NEURONS OF MOUSE MAJOR PELVIC GANGLIA}

\subsection{Introduction}

The major pelvic ganglia (MPG) contain postganglionic neurons that innervate the bladder, distal colon, and genital organs, and are a part of the urogenital pathway that controls micturition and sexual functions (Purinton, 1972; Keast, 1995). MPG receive cholinergic parasympathetic inputs from the preganglionic neurons in the sacral cord through pelvic nerve, and sympathetic cholinergic inputs from those in the lumbosacral cord through hypogastric nerve via inferior mesenteric ganglion (Kihara \& de Groat 1997; Keast, 1995).

The role of MPG in modulating transmission between the central nervous system (CNS) and the bladder has been reported to depend on the species under study. MPG in female mice has been proposed to function as spatial amplification or filtering of presynaptic inputs as there is little anatomical or functional evidence for integration of preganglionic inputs (Jobling \& Lim, 2008). However, it has been shown by Felix et al. (1998) that in male rats, there is convergence of synaptic inputs, and depression of fast synaptic EPSPs during repetitive nerve stimulation. These findings led the authors to conclude that there is integration of signals taking place in MPG neurons (Felix et al., 1998). Signaling at the MPG of cats has been reported to be more involved as it has been observed to have modulatory properties such as frequency dependent synaptic facilitation, and filtering of low frequency preganglionic activity, and amplifying intense firing during micturition in order to facilitate efficient voiding and guarding functions (de Groat \& Saum, 1976). 
Synaptic transmission at the major pelvic ganglia of rats and mice has mostly been considered to be a relay station that carries preganglionic information to the target organ with little or no synaptic alteration (Tabatabai et al., 1986; de Groat \& Booth 1980; Jobling and Lim 2008). This is reported to be due to simple morphology, small number of inputs, reliability of synaptic transmission, and lack of convergence between sympathetic and parasympathetic inputs to MPG neurons (Tabatabai.et.al, 1986; de Groat \& Booth, 1998). However, detailed characterization of the nature of synaptic transmission at the mouse MPG has not been established. Furthermore, the nature of the cholinergic receptors has not been well determined in mouse MPG.

Neuronal nicotinic acetylcholine receptors (nAChRs) are known to play a key role in mediating fast synaptic transmission at MPG (de Groat and Saum, 1976; Gallagher et al., 1982; Biasi, 2000; Park et.al, 2006). nAChRs are ligand-gated ion channels composed of five subunits to form a homopentamers or heteropentamers (Albuquerque et al, 1996; Skok, 2002; Park.et.al., 2006). Twelve genes have been identified to encode neuronal nAChR subunits: $\alpha(2-10)$ and $\beta(2-4)$ (Biasi, 2002). The main subunits found in autonomic ganglia are $\alpha 3, \alpha 4, \alpha 5, \alpha 7, \beta 3$, and $\beta 4$ (Skok, 2002). $\alpha 2-\alpha 4$ subunits can form functional channels only if co-expressed with a $\beta 2$ or $\beta 4$ subunit (Skok, 2002; Park et al, 2006).

Therefore, the goal of the present study was to investigate synaptic properties of MPG neurons, and to better characterize the physiology and receptor identities of these neurons in both male and female mice. In this study, we attempted to answer questions such as the presence or absence of frequency-dependent synaptic modulation, synaptic recruitment, 
as well as to examine contributions of various nicotinic receptor subunits to the synaptic transmission. We will investigate passive membrane properties, synaptic properties, and action potential properties of the MPG neurons by using electrophysiological and pharmacological measures. We will also quantify the abundance of mRNA transcripts for nAChRs by using real-time quantitative PCR (qPCR). It is important to understand these ganglionic properties in physiological state in order to understand first, if there are any effects of sexual dimorphism, and second, to understand if these properties are altered in disease or injuries that effect autonomic functions.

\subsection{Materials and methods}

\subsection{1 $\underline{\text { Animals }}$}

Major pelvic ganglia (MPG) were obtained from adult Swiss-Webster mice of both sexes between ages of 1-10 months. Total of 75 animals were used for both molecular analysis and in-vitro electrophysiological recordings. All experimental procedures were approved by the University of Missouri Institutional Animal Care and Use Committee and conformed to the National Institutes of Health Guide for the Care and Use of Laboratory Animals.

\subsubsection{Tissue isolation for quantitative PCR (qPCR)}

Whole MPGs from both left and right side of each animal $(\mathrm{N}=10)$ were collected in $750 \mu \mathrm{l}$ TRIzol (Invitrogen) tubes for RNA extraction. Care was taken not to include the tissues from underlying and neighboring organs. The dissected MPGs were de-sheathed and cleaned off extra tissues in a sylgard dish filled with oxygenated HEPES buffer described below. 


\subsubsection{Quantitative PCR}

Total RNA was isolated from MPGs using Trizol according to the protocol provided by the manufacturer (Invitrogen). cDNA was generated from $100 \mathrm{ng}$ total RNA primed with a mixture of oligo-dT and random hexamers that was reverse transcribed using qScript reverse transcriptase (QuantaBio). The final volume of the reverse transcription reaction was $20 \mu \mathrm{l}$ and contained a final concentration of $2.5 \mathrm{ng} / \mu 1$ random hexamers, $2.5 \mu \mathrm{M}$ oligo-dT, $40 \mathrm{U}$ of RNase inhibitor, and $200 \mathrm{U}$ of reverse transcriptase. Following heat inactivation of the enzyme, samples were diluted in ultrapure water to a final volume of $175 \mu \mathrm{l}$ before this template was used in qPCR analyses. From each cDNA pool generated from $100 \mathrm{ng}$ of total RNA, we quantified at least 15 different gene products.

We designed or modified, and independently validated primer sets for use in absolute quantitation of copy number for 7 distinct genes of interest from the mouse (see Table 2.1). Primer sets were either modified from previously validated primer sets as listed in PrimerBank, or designed de novo using Primer3 software. Primer sets are listed in Table 2.1. $\mathrm{qPCR}$ reactions consisted of primer pairs at a final concentration of $2.5 \mu \mathrm{M}$, cDNA template, and SsoAdvanced SYBR mastermix (BioRad) according to the manufacturer's instructions. Reactions were carried out on a CFXConnect (BioRad) machine with a three-step cycle of $95^{\circ} \mathrm{C}-15 \mathrm{~s}, 58^{\circ} \mathrm{C}-20 \mathrm{~s}, 72^{\circ} \mathrm{C}-20 \mathrm{~s}$, followed by a melt curve ramp from $65^{\circ} \mathrm{C}$ to $95^{\circ} \mathrm{C}$. Data were acquired during the $72^{\circ} \mathrm{C}$ step, and every $0.5^{\circ} \mathrm{C}$ of the melt curve. All reactions were run in triplicate, and the average $\mathrm{Ct}$ (cycle threshold) was used for interpolation with the standard curve to generate copy number for a given reaction. 
The units with which we express all of the qPCR data in this study is "normalized copy number per ng total RNA." All of the data are normalized relative to Glyceraldehyde 3phosphate dehydrogenase (GAPDH) and $\beta$-actin expression. A normalization factor using GAPDH for a sample $\mathrm{x}$ was calculated by the following formula:

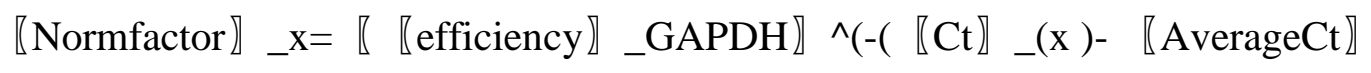
-GAPDH))

For our assay, GAPDH amplification efficiency was virtually $100 \%$, so this can be simplified as:

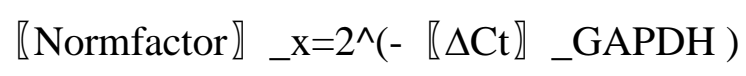

where $\llbracket \Delta \mathrm{Ct} \rrbracket \_$GAPDH is the difference between the sample GAPDH and the population average, and 2 is the base of the exponential since $100 \%$ efficiency results in a doubling of product in each cycle of PCR. This resulted in the normalized copy number for a sample $\mathrm{x}$ calculated as:

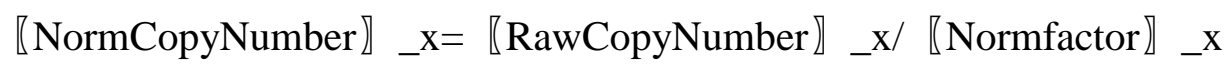

This normalization factor allows us to preserve the overall magnitude of the raw copy number, allowing for comparisons of mRNA abundance across genes. In addition, the normalization adjusts the copy number across different slices to account for differences in mRNA abundance that may occur for technical reasons, including variability in RNA extraction and reverse transcription efficiencies. 


\begin{tabular}{|c|c|c|c|c|}
\hline Gene name & Accession \# & Gene Function & Forward primer (5' to 3') & Reverse Primer (5' to 3') \\
\hline CHRNA2 & NM_144803 & Nicotinic $\alpha 2$ & $\begin{array}{l}\text { TTATCTCTGGTGTCTGCT } \\
\text { TCTGA }\end{array}$ & CCCAGCGATTGTAGCCTCC \\
\hline CHRNA3 & NM_145129 & Nicotinic $\alpha 3$ & $\begin{array}{l}\text { TCCAGTTTGAGGTGTCTA } \\
\text { TGTCT }\end{array}$ & $\begin{array}{l}\text { TGGTAGTCAGAGGGTTTCCAT } \\
\text { TT }\end{array}$ \\
\hline CHRNA4 & NM_015730 & Nicotinic $\alpha 4$ & $\begin{array}{l}\text { CTAGCAGCCACATAGAG } \\
\text { ACCC }\end{array}$ & GACAAGCCAAAGCGGACAAG \\
\hline CHRNA5 & NM_176844 & Nicotinic $\alpha 5$ & $\begin{array}{l}\text { ATCCTCTGCTGCAAAACA } \\
\text { TGA }\end{array}$ & TCCACGTCCACTAACTGAGAT \\
\hline CHRNA7 & NM_007390 & Nicotinic $\alpha 7$ & $\begin{array}{l}\text { CACATTCCACACCAACGT } \\
\text { CTT }\end{array}$ & $\begin{array}{l}\text { AAAAGGGAACCAGCGTACAT } \\
\text { C }\end{array}$ \\
\hline CHRNB2 & NM_009602.4 & Nicotinic $\beta 2$ & $\begin{array}{l}\text { ATTATCGCCTCACATGGA } \\
\text { AGC }\end{array}$ & $\begin{array}{l}\text { TGCCGTCAGCATTGTTGTATA } \\
\text { G }\end{array}$ \\
\hline CHRNB4 & NM_148944.4 & Nicotinic $\beta 4$ & $\begin{array}{l}\text { GGCAAGATCTACAGGAA } \\
\text { GCAT }\end{array}$ & ACACAATCACGAACACCCA \\
\hline$A C T B$ & NM_007393 & Beta-actin & $\begin{array}{l}\text { GATGACCCAGATCATGTT } \\
\text { TGAGACC }\end{array}$ & $\begin{array}{l}\text { AGATGGGCACAGTGTGGGTG } \\
\text { A }\end{array}$ \\
\hline GAPDH & NM_008084 & $\begin{array}{l}\text { Glyceraldehyde 3- } \\
\text { phosphate } \\
\text { dehydrogenase }\end{array}$ & $\begin{array}{l}\text { TGCACCACCACCTGCTTA } \\
\text { GC }\end{array}$ & $\begin{array}{l}\text { GGCATGGACTGTGGTCATGA } \\
\text { G }\end{array}$ \\
\hline
\end{tabular}

Table 2.1 Gene identification and primer sets used for real-time PCR reactions.

Accession numbers provided for each gene of interest. Primer sets were obtained from PrimerBank or generated de novo using PRIMER3 software. All primers sets were validated before use.

\subsubsection{Tissue isolation for in-vitro recordings}

MPGs were dissected out from the animals after euthanizing with overdose of isoflurane inhalation. The MPGs were dissected out with hypogastric, pelvic and cavernous nerves attached and pinned down in a sylgard dish with oxygenated HEPES buffer solution containing, (in $\mathrm{mM}$ ) NaCl, 146; $\mathrm{KCl}, 4.7 ; \mathrm{MgSO}_{4}, 0.6 ; \mathrm{NaHCO}_{3}, 1.6 ; \mathrm{NaH}_{2} \mathrm{PO}_{4}, 0.13$; $\mathrm{CaCl}_{2}, 2.5$; Glucose, 7.8; HEPES, 20), adjusted to $\mathrm{pH} 7.3$ at room temperature (Jobling and Lim, 2008).

\subsubsection{In-vitro electrophysiology recordings}

The dissected MPGs were de-sheathed and cleaned off from connective tissue and fat. The ganglia were pinned down on a piece of sylgard glued in a $1 \mathrm{ml}$ recording chamber 
with continuous perfusion of HEPES buffer at room temperature. Intracellular recordings were made by impaling individual MPG neurons with sharp glass electrodes. The electrodes contain intracellular solution of $3 \mathrm{M} \mathrm{KCl}$ solution and had resistances in the range between $30 \mathrm{M} \Omega-80 \mathrm{M} \Omega$. MultiClamp 700B (Molecular Devices, Sunnyvale, CA) amplifier was used for recording the signals and Digidata 1440A digitizer was used to digitize the signals at $10 \mathrm{kHz}$. The signals were acquired in Clampex 10.3 software and data was analyzed in Clampfit 10.3 program.

\subsubsection{Tests for membrane and action potential properties}

Depolarizing and hyperpolarizing current step protocols were used in bridge mode to measure the membrane and action potential properties of MPG neurons. Membrane properties such as membrane resistance and time constants are measured by intracellular hyperpolarizing current injections. Active properties such as properties of action potentials were measured from the rebound action potentials at the end of hyperpolarizing current injections. Depolarizing and hyperpolarizing current steps up to $700 \mathrm{pA}$ and -700 pA were injected directly into the cells in increments of $60 \mathrm{pA}$ and $-60 \mathrm{pA}$ steps, $400 \mathrm{~ms}$ in duration. The firing patterns of action potentials and their properties were measured. The accuracy of bridge balance was tested and ensured between recordings from each cell.

\subsubsection{Nerve stimulation and synaptic properties test}

To detect any frequency dependent modulation, a suction electrode (A-M Systems, Sequim, WA) was used to electrically stimulate pelvic nerve at various frequencies using A-M Systems Isolated Pulse Stimulator, Model 2100 (Carlsborg, WA). The pelvic nerve was stimulated at frequencies of $1 \mathrm{~Hz}, 5 \mathrm{~Hz}, 10 \mathrm{~Hz}$, and $20 \mathrm{~Hz}$ repetitive stimuli with 
pulse duration of $0.5 \mathrm{~ms}$. Intracellular recording electrodes were used to measure excitatory postsynaptic synaptic potential (EPSP) responses from individual neurons to pelvic nerve stimulation at resting membrane potential. The presence of frequencydependent synaptic modulations was measured by comparing EPSP amplitudes at various frequencies of repetitive stimuli.

To investigate synaptic recruitment from MPG neurons, we stimulated the pelvic nerve with individual pulses of $0.5 \mathrm{~ms}$ at various stimulus strengths $(1 \mathrm{~V}-30 \mathrm{~V})$. The presence of synaptic recruitment was observed by comparing the EPSP amplitudes at each stimulus intensity.

\subsubsection{Drugs Used}

Cholinergic receptor antagonists (Alomone Labs, Israel) Hexamethonium (100 $\mu \mathrm{M})$, Mecamylamine $(10 \mu \mathrm{M})$, Conotoxin AuIB $(10 \mu \mathrm{M})$, Dihydroerythroidine (DHßE) (10 $\mu \mathrm{M}, 100 \mu \mathrm{M})$, Dextromethorphan $(100 \mu \mathrm{M})$, and Atropine $(10 \mu \mathrm{M})$ were oxygenated and delivered to the preparation by perfusion for experiments investigating the effects of each antagonist on the synaptic transmission.

\subsection{9 $\underline{\text { Statistics }}$}

Data were analyzed using Sigmaplot 11.0 and by using paired and unpaired t-test, MannWhitney Rank Sum tests, One-way ANOVA and Two-way ANOVA tests. The data are presented as mean $\pm \mathrm{SD}$.

\subsection{Results}

\subsubsection{Electrophysiological properties of MPG cells}


We first measured the electrophysiological properties of MPG neurons by intracellular current injections of depolarizing and hyperpolarizing current pulses (Figure 2.1). In response to depolarizing current injections, $89 \%$ of the cells ( $\mathrm{n}=76$ out of 85$)$ responded with a single action potential and are considered 'phasic' (Figure 2.1A). $11 \%$ of the cells ( $\mathrm{n}=9$ out of 85 ) responded with continuous spikes for the whole duration of current injection and are considered to be tonic neurons (Figure 2.1B).

(A)

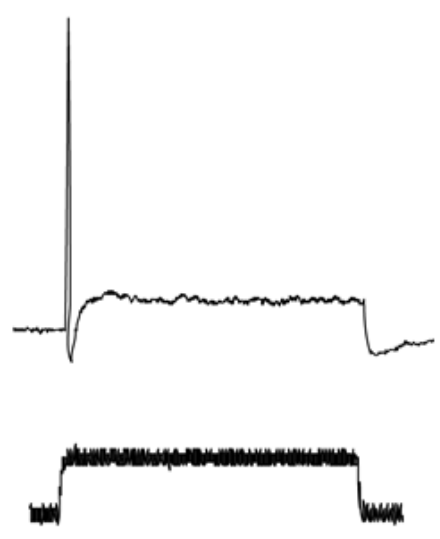

(B)

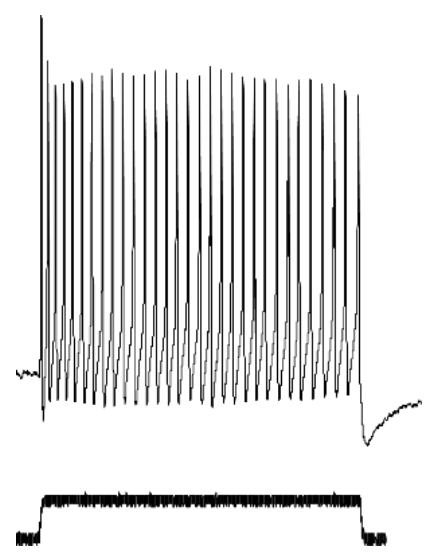

Figure 2.1 Voltage responses of MPG neurons to intracellular current injections.

(A). A representative trace of a phasic neuron that responded with a single action potential. (B) A representative trace of a tonic neuron that responded with multiple spikes throughout the current injection.

\subsubsection{Synaptic responses to nerve stimulation}

In order to study the synaptic transmission, we stimulated the pelvic nerve and measured the synaptic responses from intracellular electrodes in MPG neurons. In response to pelvic nerve stimulation, 48 percent MPG neurons ( $n=78$ out of 162) responded with suprathreshold fast synaptic potentials giving rise to action potentials whereas 52 percent of neurons ( $\mathrm{n}=84$ out of 162 ) responded with subthreshold excitatory postsynaptic 
potentials (EPSPs). Whether a particular cell responded with action potentials or EPSPs did not depend on the membrane potential of the cell (Figure 2.2).

2.3.3 Membrane properties of neurons in major pelvic ganglia

We were interested to see if the membrane properties of the cells that responded with suprathreshold potentials were different from those that responded with subthreshold EPSPs. Membrane properties described in table 2.2. were measured from responses to 
current injections of hyperpolarizing pulses of $400 \mathrm{pA}$. Resting membrane potential (RMP) among all four groups were not different in both males and females. Input resistances were measured by dividing the change in voltage by corresponding amplitude of hyperpolarizing current injections. Time constants were measured by single exponential fittings of the hyperpolarized voltage traces in response to hyperpolarizing current injections. Input resistance and cell capacitances could not be measured accurately since the measurements were made in bridge mode, and hence, we are reporting apparent values. Majority of the cells displayed a voltage sag at the beginning of the hyperpolarizing current injections. Data were analyzed using unpaired t-tests and Mann-Whitney Rank Sum tests.

Significant differences were found in apparent input resistance and apparent capacitance between the cells that responded with all-or-none action potentials and those that responded with subthreshold excitatory post-synaptic potentials (EPSPs) (Table 2.2). There were also no significant differences between males and females within either group (Table 2.2). 


\begin{tabular}{|c|c|c|c|c|c|c|c|c|c|c|c|c|c|c|c|}
\hline & \multicolumn{6}{|c|}{ Action potential generating neurons } & \multirow[b]{3}{*}{$\begin{array}{c}P P \\
\text { value }\end{array}$} & \multicolumn{7}{|c|}{ EPSP-generating neurons } & \multirow{3}{*}{$\begin{array}{c}\begin{array}{c}\text { AP vs. } \\
\text { EPSP } \\
\text { generatin } \\
\text { g neurons }\end{array} \\
P \text { value }\end{array}$} \\
\hline & \multicolumn{3}{|c|}{ Male } & \multicolumn{3}{|c|}{ Female } & & \multicolumn{3}{|c|}{ Male } & \multicolumn{4}{|c|}{ Female } & \\
\hline & $\mathbf{n}$ & $\begin{array}{l}\text { Mean } \\
\pm \text { SD }\end{array}$ & Range & $\mathbf{n}$ & $\begin{array}{l}\text { Mean } \\
\pm \text { SD }\end{array}$ & Range & & $\mathbf{n}$ & $\underset{\text { SD }}{\operatorname{Mean} \pm}$ & Range & $\mathbf{n}$ & $\begin{array}{c}\text { Mea } \\
\mathbf{n}_{ \pm} \\
\text {SD }\end{array}$ & Range & $\begin{array}{c}P \\
\text { value }\end{array}$ & \\
\hline $\begin{array}{l}\text { Resting } \\
\text { Membrane } \\
\text { Potential (RMP) } \\
(\mathrm{mV})\end{array}$ & 30 & $\begin{array}{c}-41.0 \\
\pm \\
7.8\end{array}$ & $\begin{array}{c}-25.3 \\
\text { to } \\
-62.2\end{array}$ & 42 & $\begin{array}{c}-42.3 \\
\pm \\
8.2\end{array}$ & $\begin{array}{l}-31 \text { to } \\
-71.4\end{array}$ & 0.640 & 50 & $\begin{array}{c}-42.0 \pm \\
11.16\end{array}$ & $\begin{array}{l}-25.0 \\
\text { to }-69\end{array}$ & 28 & $\begin{array}{c}-42.1 \\
\pm \\
10.8\end{array}$ & $\begin{array}{c}-20.0 \\
\text { to - } \\
65.0\end{array}$ & 0.962 & 0.867 \\
\hline $\begin{array}{l}\text { Time } \\
\text { constant(Tau) of } \\
\text { hyperpolarized } \\
\text { voltage trace }(\mathrm{ms})\end{array}$ & 24 & $\begin{array}{l}4.9 \pm \\
2.2\end{array}$ & $\begin{array}{l}1.6 \text { to } \\
12.4\end{array}$ & 16 & $\begin{array}{l}4.9 \pm \\
2.0\end{array}$ & $\begin{array}{l}1.5 \text { to } \\
10.3\end{array}$ & 0.836 & 30 & $\begin{array}{l}6.0 \pm \\
5.4\end{array}$ & $\begin{array}{l}1.7 \text { to } \\
28.4\end{array}$ & 11 & $\begin{array}{l}5.7 \pm \\
3.2\end{array}$ & $\begin{array}{l}1.7 \text { to } \\
10.6\end{array}$ & 0.803 & 0.951 \\
\hline $\begin{array}{l}\text { Percent of cells } \\
\text { with voltage sag } \\
\text { and amplitudes of } \\
\text { the sag }(\mathrm{mV})\end{array}$ & $\begin{array}{l}67 \\
\% \\
(20 / \\
30) \\
\end{array}$ & $\begin{array}{l}4.1 \pm \\
2.0\end{array}$ & $\begin{array}{c}0.4 \text { to } \\
7.7\end{array}$ & $\begin{array}{l}38 \\
\% \\
(16 / \\
42) \\
\end{array}$ & $\begin{array}{l}5.2 \pm \\
3.9\end{array}$ & $\begin{array}{l}1.8 \text { to } \\
17.8\end{array}$ & 0.656 & $\begin{array}{l}67 \\
\% \\
(29 / \\
43) \\
\end{array}$ & $\begin{array}{c}3.3 \pm \\
2.0\end{array}$ & $\begin{array}{c}0.03 \text { to } \\
6.9\end{array}$ & $\begin{array}{l}100 \\
\% \\
(11 / 1 \\
1) \\
\end{array}$ & $\begin{array}{l}4.6 \pm \\
3.3\end{array}$ & $\begin{array}{c}0.2 \text { to } \\
10.8\end{array}$ & 0.146 & 0.173 \\
\hline $\begin{array}{l}\text { Apparent input } \\
\text { resistance }(\mathrm{M} \Omega)\end{array}$ & 24 & $\begin{array}{l}86.7 \pm \\
45.2\end{array}$ & $\begin{array}{c}35.33 \\
\text { to } \\
222.03\end{array}$ & 16 & $\begin{array}{c}79 \pm \\
29.58\end{array}$ & $\begin{array}{c}28.68 \\
\text { to } \\
151.6 \\
\end{array}$ & 0.836 & 21 & $\begin{array}{l}58.66 \pm \\
18.83\end{array}$ & $\begin{array}{l}19.83 \\
\text { to } 94.3\end{array}$ & 19 & $\begin{array}{c}66.62 \\
\pm \\
25.83\end{array}$ & $\begin{array}{c}29.23 \\
\text { to } \\
117.58\end{array}$ & 0.269 & $0.010^{*}$ \\
\hline $\begin{array}{l}\text { Apparent } \\
\text { capacitance (pF) }\end{array}$ & 24 & $\begin{array}{l}60.0 \pm \\
12.99\end{array}$ & $\begin{array}{c}34.45 \\
\text { to } \\
86.67\end{array}$ & 16 & $\begin{array}{l}62 . .2 \pm \\
12.08\end{array}$ & $\begin{array}{c}46.24 \\
\text { to } \\
87.8\end{array}$ & 0.603 & 21 & $\begin{array}{l}85.4 \pm \\
63.18\end{array}$ & $\begin{array}{c}44.17 \\
\text { to } \\
289.26\end{array}$ & 19 & $\begin{array}{c}98.87 \\
\pm \frac{}{6.6}\end{array}$ & $\begin{array}{c}21.09 \\
\text { To } \\
286.31\end{array}$ & 0.448 & $0.042 *$ \\
\hline
\end{tabular}

Table 2.2 Membrane properties of the neurons in mouse major pelvic ganglia.

The table shows the comparison of membrane properties of the cells that responded with action potentials to those that responded with EPSPs. Data were analyzed by unpaired t-tests or Mann-Whitney Rank Sum tests as appropriate. * indicates $\mathrm{P}<0.05$. 
Membrane Potential and distributions of Action Potential and EPSP amplitudes

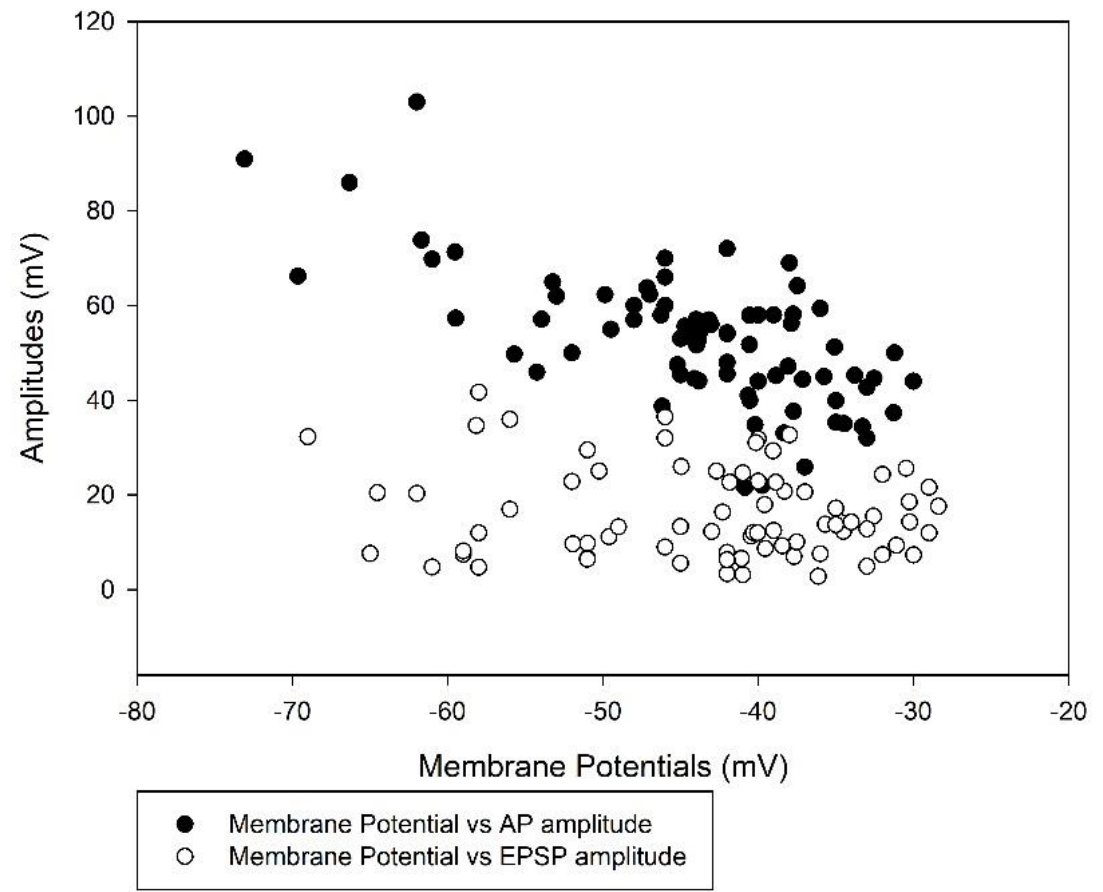

Figure 2.2 Relationship between resting membrane potentials and types of responses to nerve stimulation

Scatterplot showing the distribution of membrane potentials for those neurons that responded with all-or-none action potentials and those that responded with subthreshold EPSPs. There is no correlation between the membrane potential of the cells and the type of responses to nerve stimulations by regression analysis $\left(R^{2}=0.0775\right)$.

\subsubsection{Properties of rebound action potentials}

Rebound action potentials were observed in response to intracellular hyperpolarizing current injections of (-400) pA (Trace 2.1). Percentages of the cells that responded with rebound action potentials are: within the action-potential generating group, $79 \%(19 / 24)$ in males, $76 \%(13 / 17)$ in females, within the group that responded with subthreshold potentials, 53\% (16/30) in males, and 73\% (8/11) in females. Properties of rebound action potentials were measured by placing cursors before and after each action potential, and by using statistics program in Clampfit software. Properties of those rebound action potentials were compared between those neurons that responded with suprathreshold 
responses or action potentials and those that responded with excitatory postsynaptic voltage responses (EPSP) to pelvic nerve stimulation (Table 2.3, Figure 2.3). Significant differences were observed in majority of the rebound action potential properties between those two groups. There were no differences between males and females within either of the groups (Table 2.3, Figure 2.3). 


\begin{tabular}{|c|c|c|c|c|c|c|c|c|c|c|c|c|c|c|c|}
\hline & \multicolumn{6}{|c|}{ Action potential generating neurons } & & \multicolumn{7}{|c|}{ EPSP-generating neurons } & \multirow{3}{*}{$\begin{array}{c}\text { AP vs. } \\
\text { EPSP } \\
\text { generati } \\
\text { ng } \\
\text { neurons } \\
P \text { value }\end{array}$} \\
\hline & \multicolumn{3}{|c|}{ Male } & \multicolumn{3}{|c|}{ Female } & & \multicolumn{3}{|c|}{ Male } & \multicolumn{4}{|c|}{ Female } & \\
\hline & $\mathrm{n}$ & $\begin{array}{l}\text { Mean } \pm \\
\text { SD }\end{array}$ & Range & $\mathrm{n}$ & $\begin{array}{l}\text { Mean } \pm \\
\text { SD }\end{array}$ & Range & $\begin{array}{c}P \\
\text { value }\end{array}$ & $\mathrm{n}$ & $\begin{array}{c}\text { Mean } \pm \\
\text { SD }\end{array}$ & Range & $\mathrm{n}$ & $\begin{array}{l}\text { Mean } \pm \\
\text { SD }\end{array}$ & Range & $\begin{array}{c}P \\
\text { value }\end{array}$ & \\
\hline $\begin{array}{l}\text { Resting Membrane } \\
\text { Potential (RMP) (mV }\end{array}$ & 18 & $\begin{array}{c}-37.75 \\
\pm \\
4.95\end{array}$ & $\begin{array}{c}-46.73 \\
\text { to } \\
-30.34\end{array}$ & 15 & $\begin{array}{c}-36.35 \\
\pm \\
5.012\end{array}$ & $\begin{array}{c}-43.40 \\
\text { to } \\
-23.04\end{array}$ & 0.426 & 15 & $\begin{array}{c}-32.53 \\
\pm \\
4.31\end{array}$ & $\begin{array}{c}-41.07 \\
\text { to } \\
-24.86\end{array}$ & 7 & $\begin{array}{l}-34.69 \\
\pm \\
4.40\end{array}$ & $\begin{array}{c}-39.85 \\
\text { to } \\
-28.60\end{array}$ & 0.289 & $0.004 * *$ \\
\hline Peak amplitude (mV) & 18 & $\begin{array}{c}54.46 \\
\pm \\
9.131 \\
\end{array}$ & $\begin{array}{c}33.244 \\
\text { to } \\
66.101\end{array}$ & 15 & $\begin{array}{c}51.338 \\
\pm \\
11.397 \\
\end{array}$ & $\begin{array}{c}21.605 \\
\text { to } \\
67.799\end{array}$ & 0.387 & 15 & $\begin{array}{l}44.229 \pm \\
9.18\end{array}$ & $\begin{array}{c}30.793 \\
\text { to } \\
60.948\end{array}$ & 7 & $\begin{array}{l}45.27 \\
\pm 10.00 \\
9 \\
\end{array}$ & $\begin{array}{c}35.471 \\
\text { to } \\
60.365\end{array}$ & 0.813 & $0.003 * *$ \\
\hline $\begin{array}{l}\text { Antipeak/Afterhyper } \\
\text { polarization } \\
\text { amplitude (AHP) } \\
(\mathrm{mV})\end{array}$ & 18 & $\begin{array}{c}-14.028 \\
\pm \\
4.530\end{array}$ & $\begin{array}{l}- \\
22.594 \\
\text { to } \\
-7.041 \\
\end{array}$ & 15 & $\begin{array}{c}- \\
11.697 \\
\pm \\
5.535 \\
\end{array}$ & $\begin{array}{c}-21.414 \\
\text { to } \\
-0.856\end{array}$ & 0.193 & 15 & $\begin{array}{l}-7.9 \\
\pm 4.12\end{array}$ & $\begin{array}{c}-16.598 \\
\text { to } \\
-2.637\end{array}$ & 7 & $\begin{array}{l}-8.28 \\
\pm 4.802\end{array}$ & $\begin{array}{c}-15.776 \\
\text { to } \\
-1.181\end{array}$ & 0.865 & $\begin{array}{l}<0.001 * * \\
*\end{array}$ \\
\hline Half-width (ms) & 18 & $\begin{array}{c}3.583 \\
\pm \\
0.694 \\
\end{array}$ & $\begin{array}{c}2.459 \\
\text { to } \\
4.673 \\
\end{array}$ & 15 & $\begin{array}{c}3.506 \\
\pm\end{array}$ & $\begin{array}{c}2.726 \\
\text { to } \\
6.325 \\
\end{array}$ & 0.270 & 15 & $\begin{array}{l}4.6 \pm \\
1.43\end{array}$ & $\begin{array}{c}2.675 \\
\text { to } \\
8.046\end{array}$ & 7 & $\begin{array}{l}4.28 \pm \\
0.676\end{array}$ & $\begin{array}{c}3.782 \\
\text { to } \\
5.647\end{array}$ & 0.534 & $0.025^{*}$ \\
\hline $\begin{array}{l}\text { Max rise } \\
\text { slope }(\mathrm{mV} / \mathrm{ms})\end{array}$ & 18 & $\begin{array}{c}49.072 \\
\pm 17.795\end{array}$ & $\begin{array}{c}18.750 \\
\text { to } \\
83.984\end{array}$ & 15 & $\begin{array}{c}43.065 \\
\pm \\
20.706\end{array}$ & $\begin{array}{c}14.160 \\
\text { to } \\
85.061\end{array}$ & 0.377 & 15 & $\begin{array}{l}25.0 \\
\pm 17.91\end{array}$ & $\begin{array}{c}13.110 \\
\text { to } \\
77.134\end{array}$ & 7 & $\begin{array}{l}33.07 \\
\pm 20.6\end{array}$ & $\begin{array}{c}14.974 \\
\text { to } \\
38.603\end{array}$ & 0.888 & $0.001 * *$ \\
\hline $\begin{array}{l}\text { Max decay slope } \\
(\mathrm{mV} / \mathrm{ms})\end{array}$ & 18 & $\begin{array}{c}-18.7 \\
\pm 5.223\end{array}$ & $\begin{array}{c}- \\
29.063 \\
\text { to } \\
- \\
10.976\end{array}$ & 15 & $\begin{array}{r}- \\
15.702 \\
+4.857\end{array}$ & $\begin{array}{l}-25.336 \\
\text { to } \\
-7.813\end{array}$ & 0.100 & 15 & $\begin{array}{l}-11.6 \pm \\
5.029\end{array}$ & $\begin{array}{c}-22.311 \\
\text { to } \\
-4.289\end{array}$ & 7 & $\begin{array}{l}-12.173 \\
\pm 3.199\end{array}$ & $\begin{array}{c}-17.253 \\
\text { to } \\
-7.047\end{array}$ & 0.785 & $\begin{array}{l}<0.001 * * \\
*\end{array}$ \\
\hline Rise time (ms) & 18 & $\begin{array}{r}1.726 \\
\pm 1.393\end{array}$ & $\begin{array}{c}0.966 \\
\text { to } \\
11.537 \\
\end{array}$ & 15 & $\begin{array}{l}2.071 \pm \\
1.353\end{array}$ & $\begin{array}{c}0.968 \\
\text { to } \\
9.615 \\
\end{array}$ & 0.678 & 15 & $\begin{array}{l}2.24 \pm \\
1.67\end{array}$ & $\begin{array}{c}0.936 \\
\text { to } \\
11.422 \\
\end{array}$ & 7 & $\begin{array}{c}2.59 \\
\pm 1.83\end{array}$ & $\begin{array}{c}1.143 \\
\text { to } \\
6.644 \\
\end{array}$ & 0.622 & 0.246 \\
\hline Rise slope (mV/ms) & 18 & $\begin{array}{c}24.736 \pm \\
14.704\end{array}$ & $\begin{array}{c}1.896 \\
\text { to } \\
54.683\end{array}$ & 15 & $\begin{array}{c}23.403 \\
\pm \\
16.002\end{array}$ & $\begin{array}{c}2.933 \\
\text { to } \\
57.747\end{array}$ & 0.805 & 15 & $\begin{array}{l}13.08 \\
+9.3\end{array}$ & $\begin{array}{c}2.241 \\
\text { to } \\
55.402\end{array}$ & 7 & $\begin{array}{l}13.08 \\
\pm 9.5\end{array}$ & $\begin{array}{c}3.982 \\
\text { to } \\
30.871\end{array}$ & 0.888 & 0.060 \\
\hline Decay time (ms) & 18 & $\begin{array}{c}2.940 \\
\pm \\
0 . \frac{6}{643}\end{array}$ & $\begin{array}{c}2.093 \\
\text { to } \\
4.162\end{array}$ & 15 & $\begin{array}{c}3.392 \\
+0.841\end{array}$ & $\begin{array}{c}2.225 \\
\text { to } \\
5.276\end{array}$ & 0.090 & 15 & $\begin{array}{l}4.17 \pm \\
1.69\end{array}$ & $\begin{array}{c}2.307 \\
\text { to } \\
8.270\end{array}$ & 7 & $\begin{array}{c}3.74 \\
\pm 1.02\end{array}$ & $\begin{array}{c}2.214 \\
\text { to } \\
5.343\end{array}$ & 0.541 & $0.025^{*}$ \\
\hline $\begin{array}{l}\text { Decay slope } \\
(\mathrm{mV} / \mathrm{ms})\end{array}$ & 18 & $\begin{array}{c}-15.8 \\
\pm 4.066\end{array}$ & $\begin{array}{l}- \\
23.646 \\
\text { to } \\
-9.085\end{array}$ & 15 & $\begin{array}{r}-13.49 \\
\pm 4.796\end{array}$ & $\begin{array}{c}-20.485 \\
\text { to } \\
-3.410\end{array}$ & 0.130 & 15 & $\begin{array}{l}-10.34 \pm \\
4.68\end{array}$ & $\begin{array}{c}-19.892 \\
\text { to } \\
-3.336\end{array}$ & 7 & $\begin{array}{l}-10.58 \pm \\
3.06\end{array}$ & $\begin{array}{c}-14.880 \\
\text { to } \\
-5.442\end{array}$ & 0.906 & $\begin{array}{l}<0.001 * * \\
*\end{array}$ \\
\hline
\end{tabular}

Table 2.3 Properties of rebound action potentials.

Rebound action potential properties from action potential-generating and EPSP-generating neurons compared using unpaired t-tests or Mann-Whitney Rank Sum tests as appropriate. * indicates $\mathrm{P}<0.05$, ** indicates $\mathrm{P}<0.01$ and $* * *$ indicates $\mathrm{P}<0.001$. 
Resting membrane potential

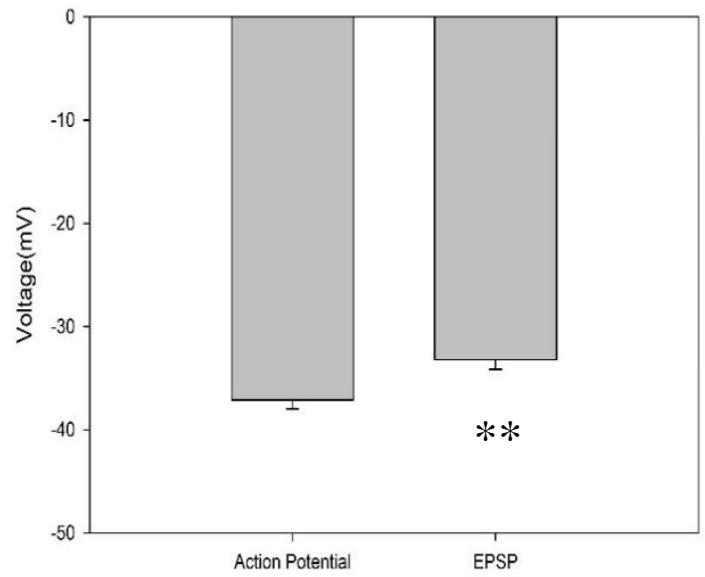

Antipeak(Afterhyperpolarization) amplitude

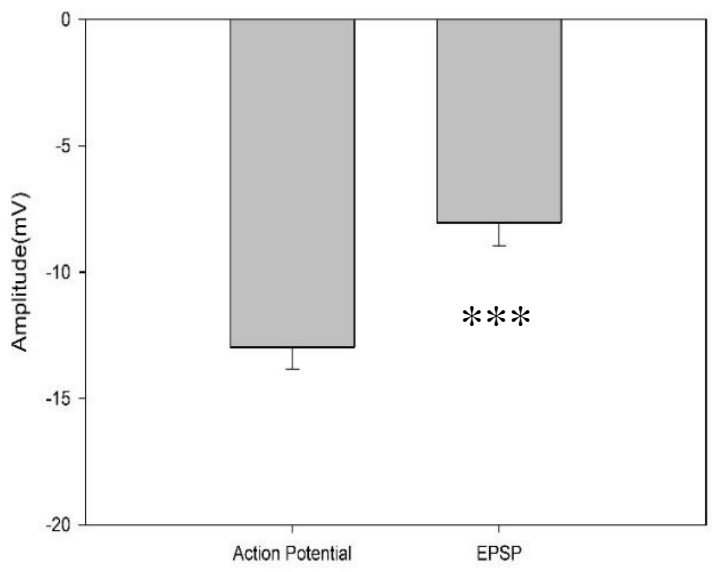

Max rise slope

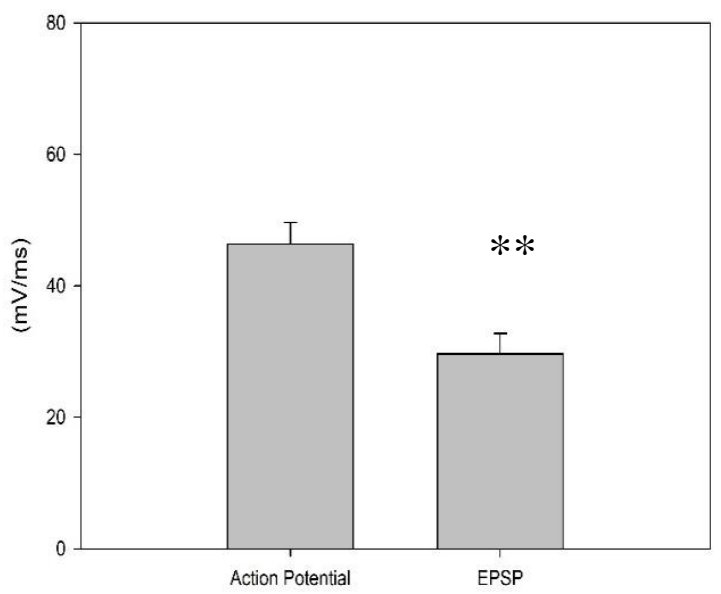

Peak amplitude

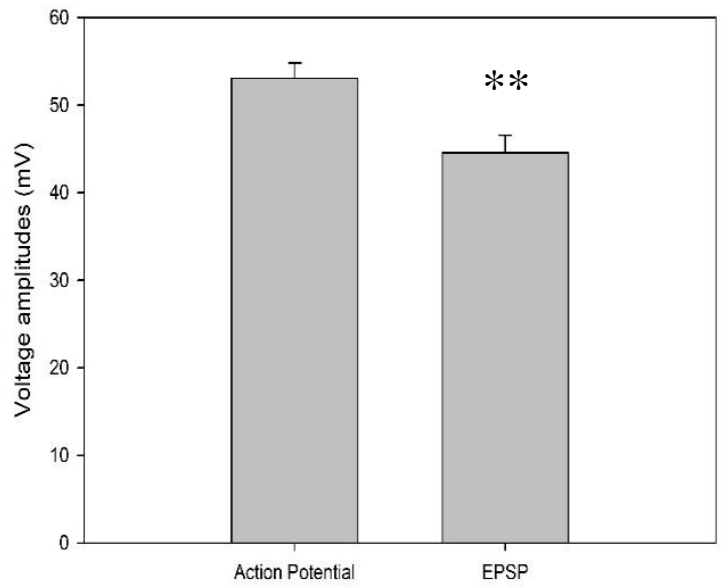

Half-width

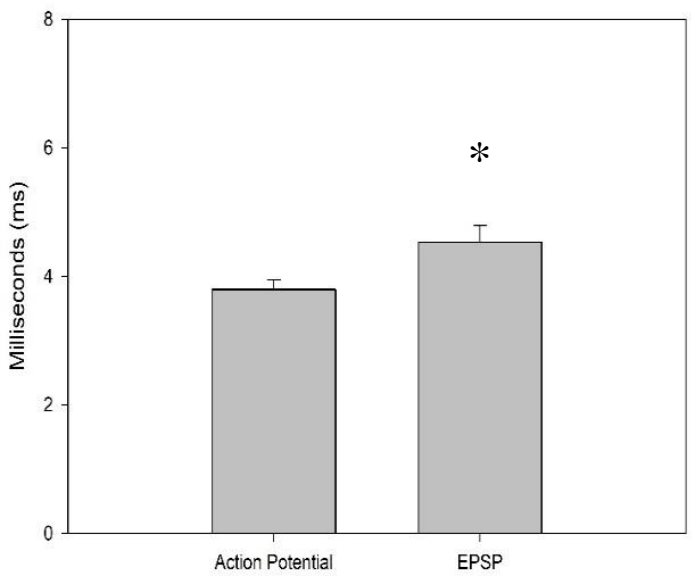

Max decay slope

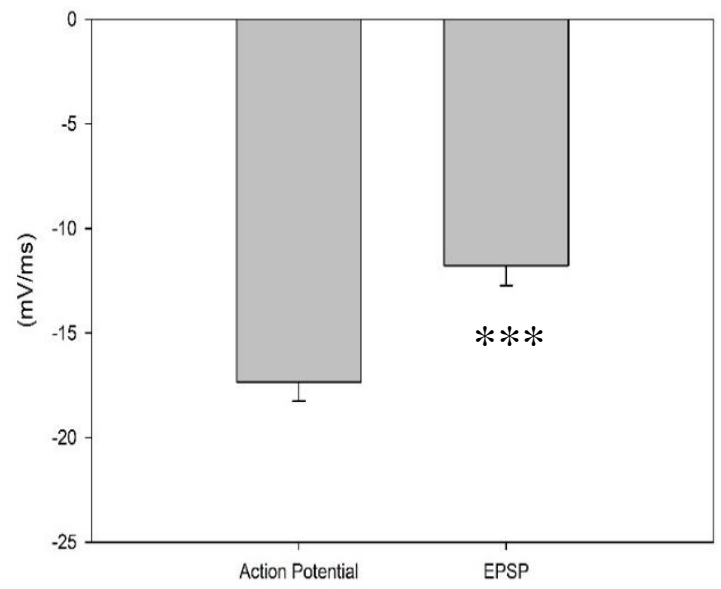



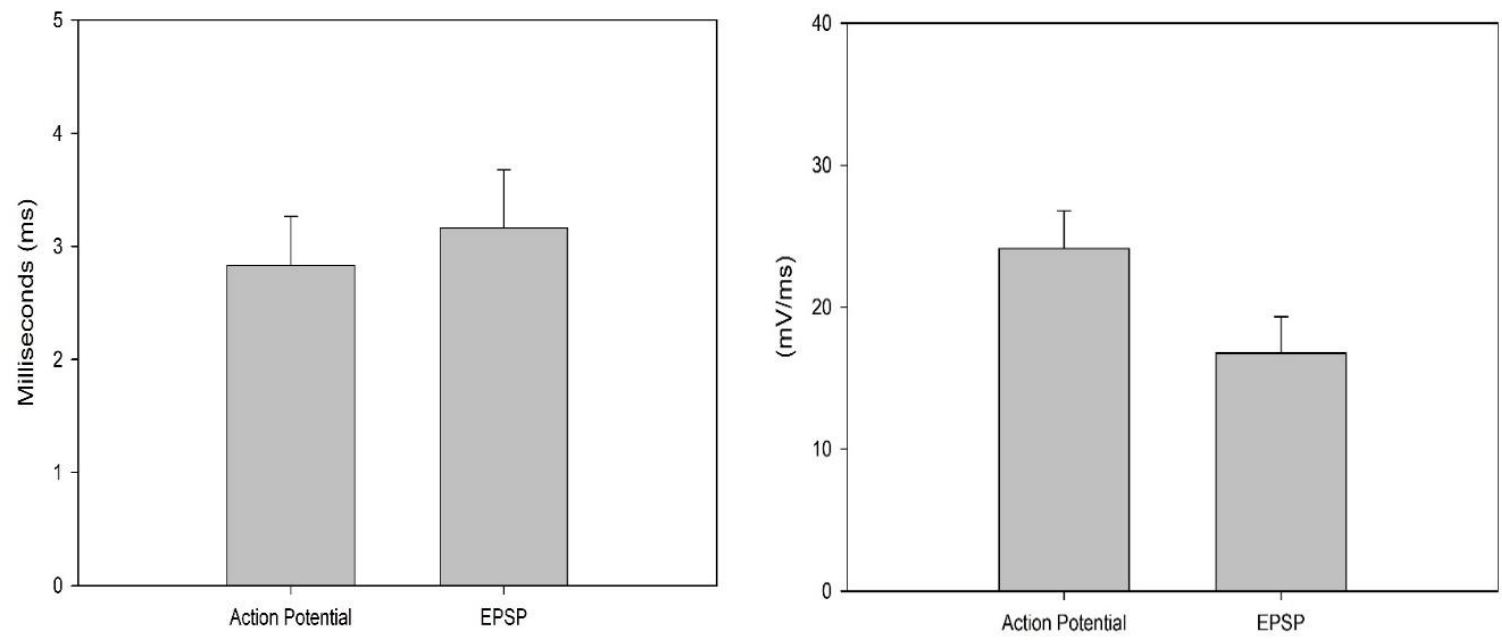

Decay time

Decay slope
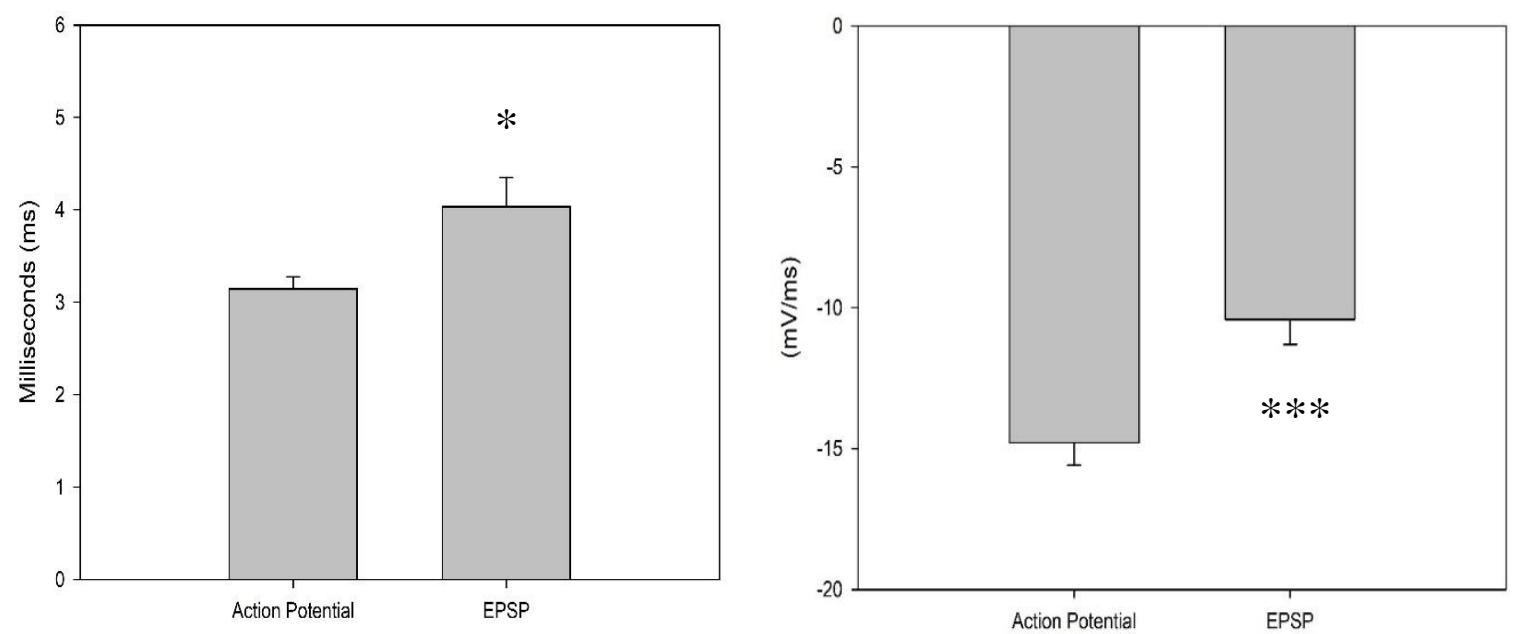

Figure 2.3 Significant differences between properties of rebound action potentials were observed (see table 2.3) from action potential and EPSP generating neurons.

Graphs show comparison of parameters from the rebound action potentials. Data were analyzed using unpaired t-tests or Mann-Whitney Rank Sum tests as appropriate. * indicates $\mathrm{P}<0.05$, ** indicates $\mathrm{P}<0.01$ and $* * *$ indicates $\mathrm{P}<0.001$. 


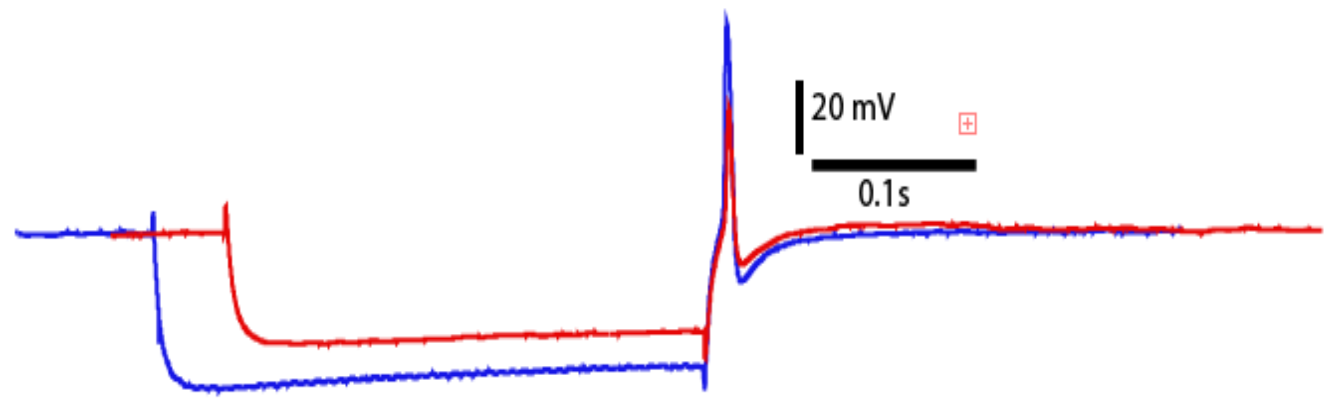

Trace 2.1 Example traces showing rebound action potentials from action potentialgenerating in blue and from EPSP-generating neurons in red.

$\underline{\text { Pelvic nerve stimulation-evoked suprathreshold synaptic potentials }}$

Various shapes and amplitudes of suprathreshold synaptic potentials were elicited by pelvic nerve stimulation (Figure 2.4). Representative traces shown in (A) and (B) of figure 2.4 show EPSPs reaching threshold and giving rise to action potentials, whereas (C), (D) and (E) shows large amplitude, fast synaptic responses which do not have threshold inflection on the rising phase. Percentages of response types observed are 36.8 $\%(25$ out of 68 ) of type A, $17.65 \%$ (12 out of 68 ) of type B, $14.7 \%(10$ out of 68$)$ of type C, $17.65 \%$ (12 out of 68 ) of type D, and $13.23 \%$ (9 out of 68 ) of type E. 


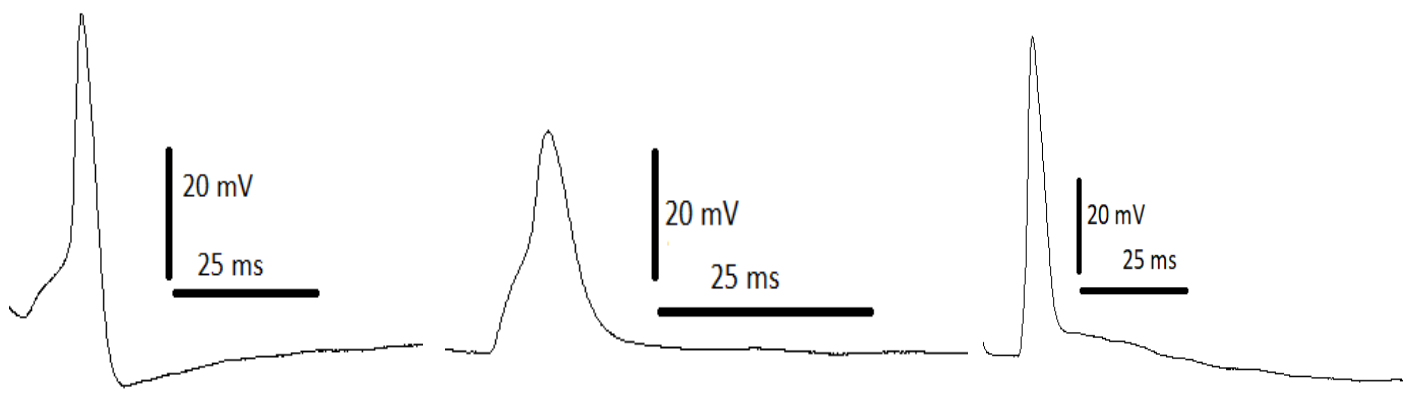

(A)

(B)

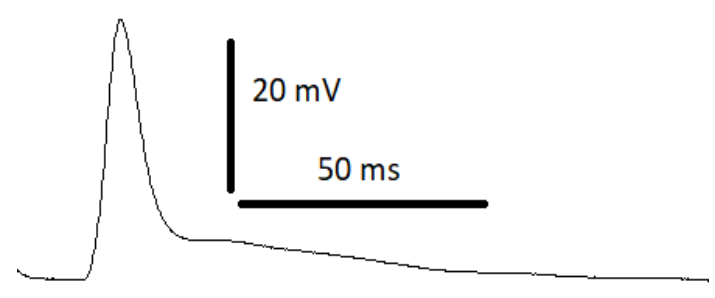

(D)

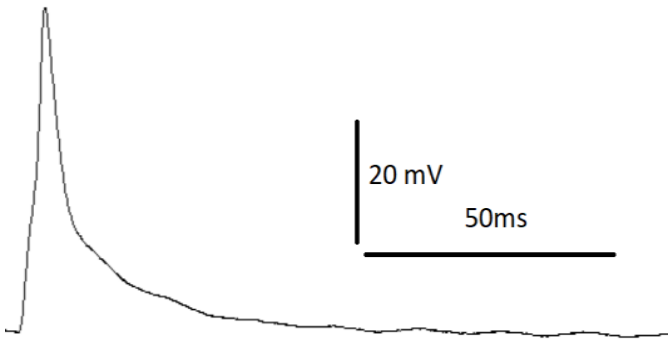

(E)

Figure 2.4 Representative traces showing various shapes and sizes of fast synaptic responses in response to pelvic nerve stimulation.

(A) An action potential with a threshold inflection and afterhyperpolarization (AHP). (B) An action potential with a threshold inflection but without afterhyperpolarization. (C) A fast depolarization without threshold inflection and with a small afterdepolarization. (D) and (E) represent traces of fast depolarization responses with different shapes in decay phases possibly with different decay time constants.

The action potentials responded in all-or-none fashion to pelvic nerve stimulation at threshold voltage of stimulation. These suprathreshold potentials were seen in $48 \%$ (78 out of 162) of the neurons tested. The properties of action potentials are described in table 2.4. Subthreshold EPSP responses were observed in $52 \%$ (84 out of 162) of the neurons tested, and their properties are described in table 2.5. 


\begin{tabular}{|c|c|c|c|c|c|c|c|}
\hline & \multicolumn{3}{|c|}{ Males } & \multicolumn{3}{|c|}{ Females } & \\
\hline & $\mathrm{n}$ & $\begin{array}{c}\text { Mean } \pm \\
\text { SD }\end{array}$ & Range & $\mathrm{n}$ & $\begin{array}{l}\text { Mean } \pm \\
\text { SD }\end{array}$ & Range & $\begin{array}{c}P \\
\text { value }\end{array}$ \\
\hline $\begin{array}{c}\text { Resting Membrane } \\
\text { Potential (RMP) (mV) }\end{array}$ & 30 & $\begin{array}{l}-41.0 \pm \\
7.8\end{array}$ & $\begin{array}{l}-25.3 \text { to } \\
-62.2\end{array}$ & 42 & $\begin{array}{c}-42.3 \\
\pm \\
8.2 \\
\end{array}$ & $\begin{array}{l}-31 \text { to } \\
-71.4\end{array}$ & 0.640 \\
\hline $\begin{array}{c}\text { Action Potential } \\
\text { amplitude measured } \\
\text { from RMP }(\mathrm{mV})\end{array}$ & 30 & $\begin{array}{c}52.8 \pm \\
15.0\end{array}$ & 27.8 to 104.0 & 42 & $\begin{array}{l}54.7 \pm \\
12.2\end{array}$ & $\begin{array}{c}29.0 \text { to } \\
91.5\end{array}$ & 0.461 \\
\hline $\begin{array}{l}\text { Action Potential half- } \\
\text { width (ms) }\end{array}$ & 30 & $5.0 \pm 1.6$ & 3.0 to 8.5 & 42 & $5.6+2.2$ & $\begin{array}{c}3.2 \text { to } \\
16.5 \\
\end{array}$ & 0.130 \\
\hline $\begin{array}{l}\text { Afterhyperpolarization } \\
\text { (AHP) amplitude (mV) }\end{array}$ & 27 & $-5.1 \pm 4.2$ & -14 to -0.07 & 31 & $-4.3+5.1$ & $\begin{array}{c}-20.7 \text { to }- \\
0.1 \\
\end{array}$ & 0.218 \\
\hline $\begin{array}{l}\text { Threshold for action } \\
\text { potential generation } \\
(\mathrm{mV})\end{array}$ & 12 & $20.6 \pm 6.0$ & 10.4 to 32.5 & 19 & $21.2 \pm 5.5$ & 10 to 33 & 0.757 \\
\hline Rise Tau (ms) & 30 & $\begin{array}{c}10.9 \pm \\
17.7 \\
\end{array}$ & 1.3 to 88.3 & 42 & $9.3+15.6$ & $\begin{array}{l}1.1 \text { to } \\
90.4\end{array}$ & 0.587 \\
\hline Decay Tau (ms) & 29 & $\begin{array}{l}31.7 \pm \\
51.5 \\
\end{array}$ & 2.2 to 252.7 & 42 & $\begin{array}{c}31.1 \pm \\
97.4 \\
\end{array}$ & $\begin{array}{l}2.1 \text { to } \\
627.8 \\
\end{array}$ & 0.226 \\
\hline
\end{tabular}

Table 2.4 Properties of nerve-stimulated action potentials. 


\begin{tabular}{|c|c|c|c|c|c|c|c|}
\hline & \multicolumn{3}{|c|}{ Males } & \multicolumn{3}{|c|}{ Females } & \multirow[b]{2}{*}{$P$ value } \\
\hline & $\mathrm{n}$ & Mean \pm SD & Range & $\mathrm{n}$ & $\begin{array}{l}\text { Mean } \pm \\
\text { SD }\end{array}$ & Range & \\
\hline $\begin{array}{c}\text { Resting Membrane } \\
\text { Potential (RMP) (mV) }\end{array}$ & 50 & $\begin{array}{c}-42.0 \pm \\
11.16\end{array}$ & -25.0 to -69 & 26 & $\begin{array}{c}-43.6 \pm \\
9.7 \\
\end{array}$ & $\begin{array}{l}-30.5 \text { to }- \\
64.5\end{array}$ & 0.962 \\
\hline $\begin{array}{c}\text { EPSP amplitude } \\
\text { measured from RMP } \\
(\mathrm{mV})\end{array}$ & 45 & $16.01 \pm 9.9$ & 2.8 to 36.5 & 26 & $16.8 \pm 8.8$ & $\begin{array}{c}3.4 \text { to } \\
41.7\end{array}$ & 0.447 \\
\hline EPSP rise time (ms) & 24 & $3.3 \pm 1.2$ & 0.7 to 5.4 & 7 & $3.8 \pm 0.8$ & 2.9 to 5.1 & 0.330 \\
\hline EPSP half-width (ms) & 16 & $13.6 \pm 3.4$ & 6.3 to 17.3 & 5 & $16.8 \pm 4.7$ & $\begin{array}{c}12.6 \text { to } \\
25.0\end{array}$ & 0.341 \\
\hline EPSP Area (mV.ms) & 23 & $217 \pm 156$ & 28.5 to 612.5 & 5 & $\begin{array}{c}259.0 \pm \\
167.2\end{array}$ & $\begin{array}{l}66.3 \text { to } \\
391.4\end{array}$ & 0.810 \\
\hline
\end{tabular}

Table 2.5 Properties of nerve-stimulated EPSPs. 


\subsubsection{Frequency-dependent depression of EPSPs}

In next experiments, we explored if there was frequency-dependent modulation or facilitation of synaptic transmission at the MPG. Pelvic nerve was stimulated at frequencies of $2 \mathrm{~Hz}, 5 \mathrm{~Hz}, 10 \mathrm{~Hz}$ and $20 \mathrm{~Hz}$, and the number and amplitude of the resulting synaptic responses were measured at minimum voltage to elicit a synaptic response (Figure 2.5A). Significant depressions of EPSP amplitudes were observed at all frequencies when normalized pulse 1 was compared to normalized amplitudes of both pulse 2 and pulse 5 at all frequencies. Responses at higher frequencies $(10 \mathrm{~Hz}$ and $20 \mathrm{~Hz})$ showed greater magnitude of depression of responses compared to those at $2 \mathrm{~Hz}$ and $5 \mathrm{~Hz}$ (Figue 5.B). Data were analyzed using paired t-tests and the $\mathrm{P}$ values are, at $2 \mathrm{~Hz}, \mathrm{P} 1 \mathrm{vs}$. $\mathrm{P} 2=0.003$, $\mathrm{P} 1$ vs. $\mathrm{P} 5=0.014$; at $5 \mathrm{~Hz}, \mathrm{P} 1$ vs. $\mathrm{P} 2=0.009$, $\mathrm{P} 1$ vs. $\mathrm{P} 5=<0.001$; at $10 \mathrm{~Hz}, \mathrm{P} 1$ vs. $\mathrm{P} 2=<0.001$, P1vs. $\mathrm{P} 5=<0.001$; and at $20 \mathrm{~Hz}, \mathrm{P} 1$ vs. $\mathrm{P} 2=<0.001$, $\mathrm{P} 1$ vs. $\mathrm{P} 5=<0.001$ (Figure 2.5B).In the cells that responded with action potentials, action potentials were reduced to EPSPs with irregular patterns of responses at $20 \mathrm{~Hz}$. Significant depression of synaptic responses was observed in $75 \%$ ( $\mathrm{n}=24$ out of 34 ) of the neurons tested. 


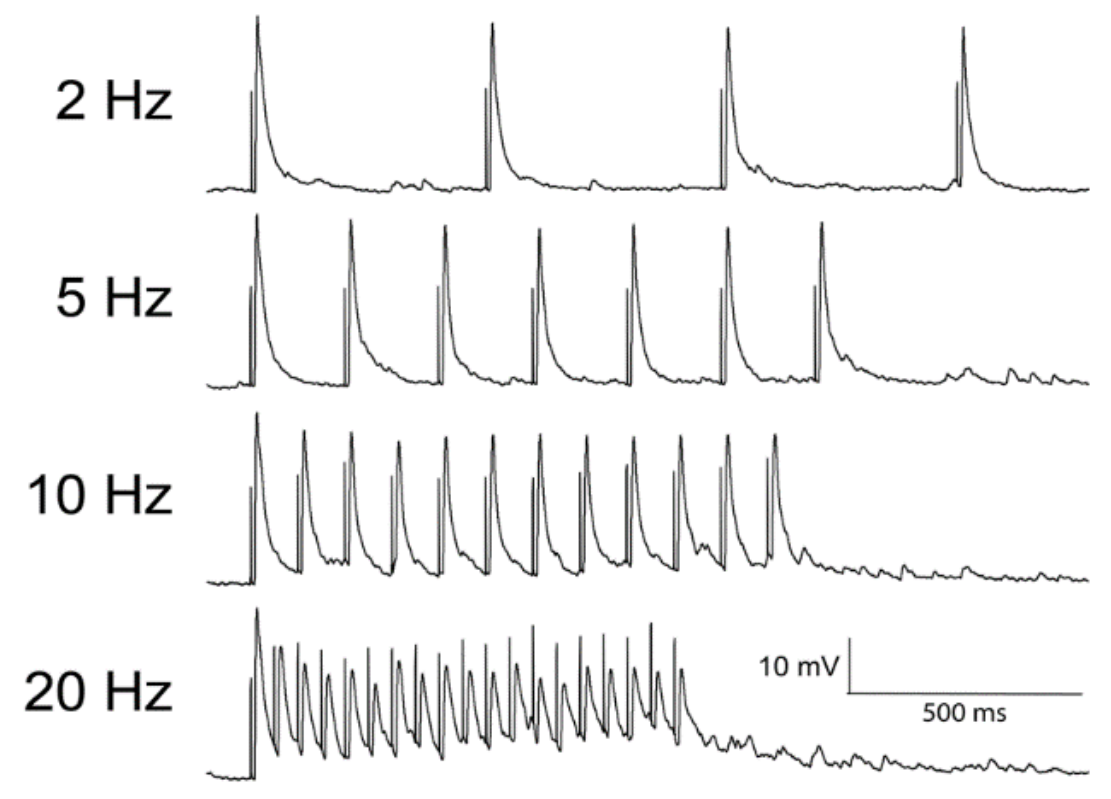

Depression of synaptic responses at various frequencies of pelvic nerve stimulation

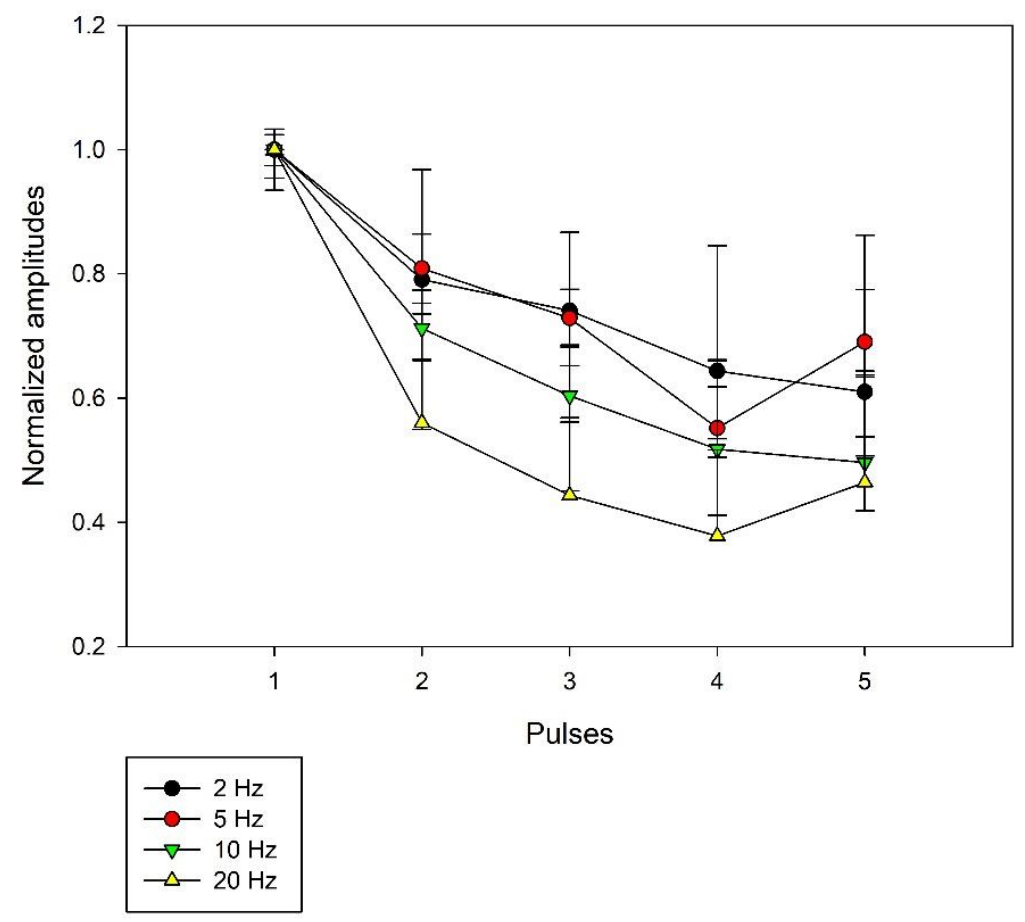

Figure 2.5 Frequency-dependent synaptic depression was observed at higher frequencies of pelvic nerve stimulation.

EPSP amplitudes of MPG neurons $(n=24)$ in response to repetitive stimulation of pelvic nerve at 2, 5, 10 and $20 \mathrm{~Hz}$. (Panel A) Representative traces showing EPSP responses at various frequencies. Depression of amplitudes could be seen at $10 \mathrm{~Hz}$ and $20 \mathrm{~Hz}$. (Panel B) EPSP amplitudes were plotted for each frequency. P1 to P5 correspond to the first 5 pulses of repetitive stimulation. The EPSP amplitudes from P2 to P5 were normalized relative to the first pulse. (Pulse duration $=0.5 \mathrm{~ms}$, Inter-pulse interval $=200 \mathrm{~ms}$ ) 
Decreasing number of responses to nerve stimulation was also seen in neurons that responded with all-or-none action potentials $(\mathrm{n}=20)$. The fidelity of responses to nerve stimulation decreased with increasing frequency of stimulation. Figure 2.6 shows highest response rate (100\%) of action potentials at $2 \mathrm{~Hz}$ but significantly decreased to $12 \%$ at $20 \mathrm{~Hz}$. At lower frequencies, the neurons had one-to-one response rate to stimuli whereas at frequencies of $10 \mathrm{~Hz}$ and higher, the neurons stopped responding consistently to the stimuli.

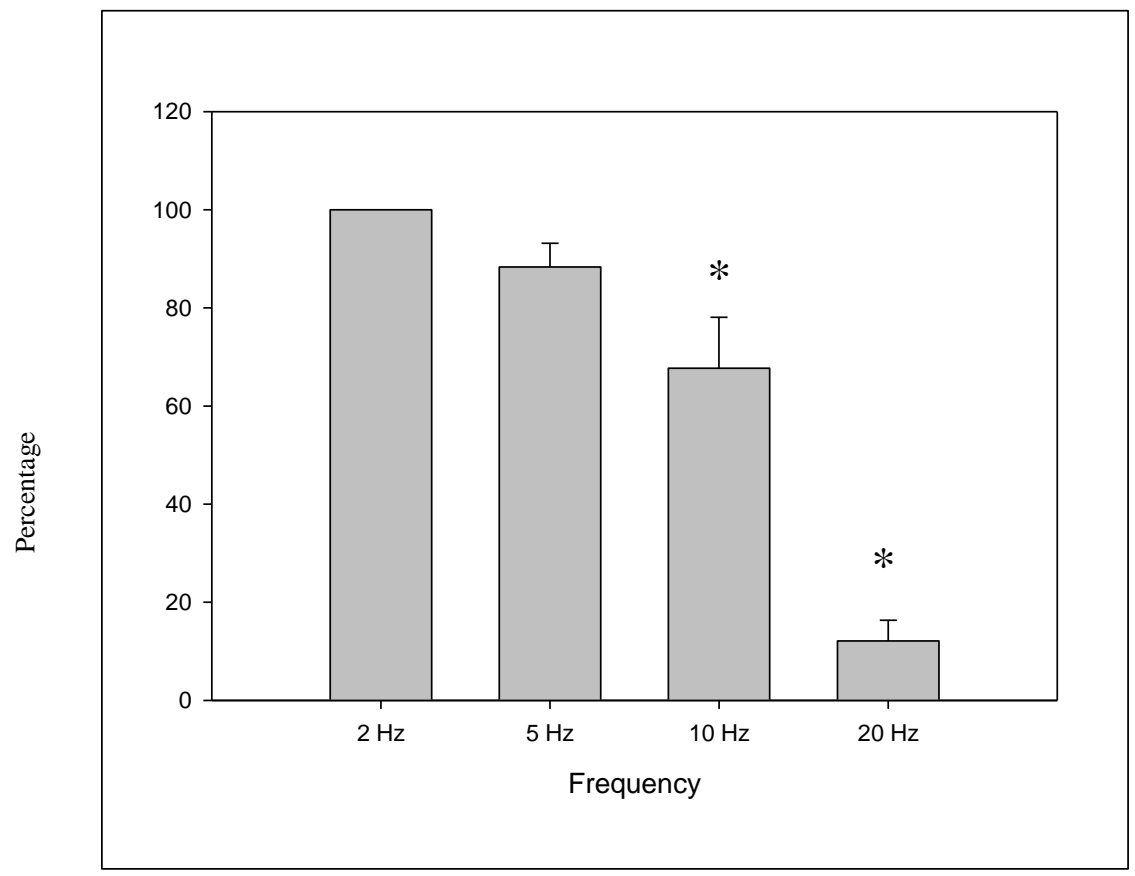

Figure 2.6 Decreased in reliability of action potential firing was observed at higher frequencies of stimulation.

The graph shows decreasing number of action potentials at higher frequencies during repetitive stimulation of pelvic nerve $(n=20)$. The bars indicate the percentage of action potential responses recorded in response to $5 \mathrm{~s}$ of repetitive stimulation of pelvic nerve. The percentages were calculated by obtaining the ratio between the number of responses and the number of stimuli applied. Error bars indicate standard error. Asterisks indicate significant decrease in percentage of responses from the percentage at $2 \mathrm{~Hz}$ using one-way ANOVA tests.

(* indicates $P<0.05$ ) 


\subsubsection{Spatial Summation}

In order to investigate the presence of synaptic recruitment at the MPG neurons, pelvic nerve was stimulated at various strengths of stimulation $(1 \mathrm{~V}-30 \mathrm{~V})$, and the amplitudes of synaptic responses were measured. $53 \%$ of the cells tested ( $n=16$ out of 30 neurons) showed increase in amplitudes of post-synaptic potentials in response to single pulse stimulations of the pelvic nerve at increasing strengths of stimuli at each pulse. Out of those neurons that showed increase in EPSP amplitudes at increasing magnitudes of stimulation, 11 out of 16 neurons showed graded increase in amplitudes from smallamplitude EPSPs to large-amplitude EPSPs. 5 out of 16 neurons showed graded increase from small EPSP to action potentials after the stimulating voltage was strong enough to reach the threshold. Increasing EPSP amplitudes range from 13\% of the initial amplitude to $1374 \%$ with increasing stimulus strengths. Figure 2.7 shows examples of a cell with graded increase in EPSP amplitude (2.7A) and another cell which did not respond at lower stimulus strength but responded with an EPSP and eventually with an action potential at higher magnitudes of stimulation (2.7B). The membrane potentials were not changing at the times that the measurements were taken from initial to the final pulses. 
(A)

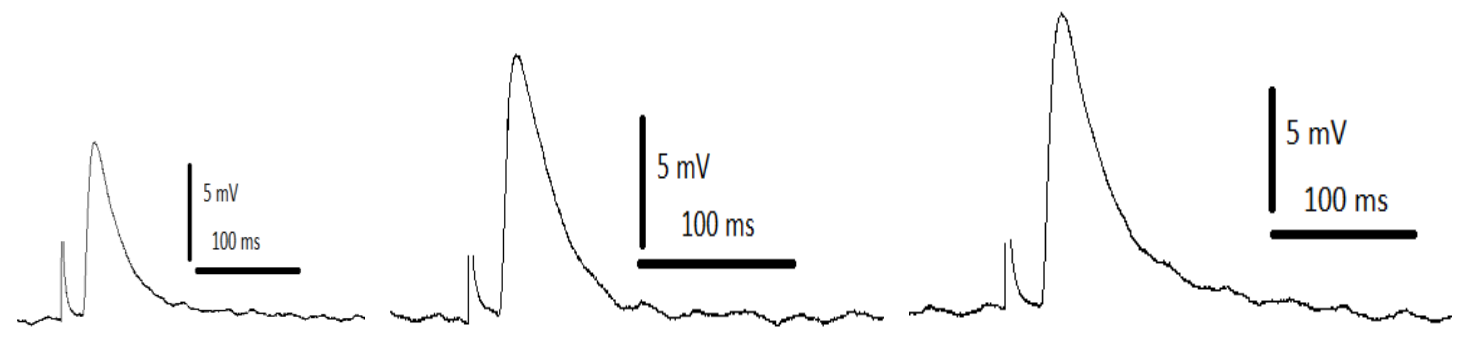

(i)

(ii)

(iii)

(B)

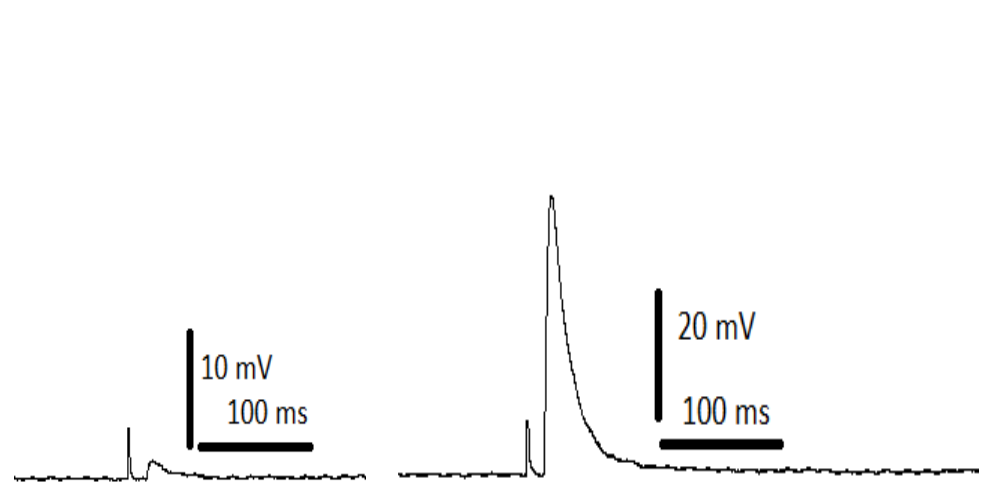

(i)

(ii)

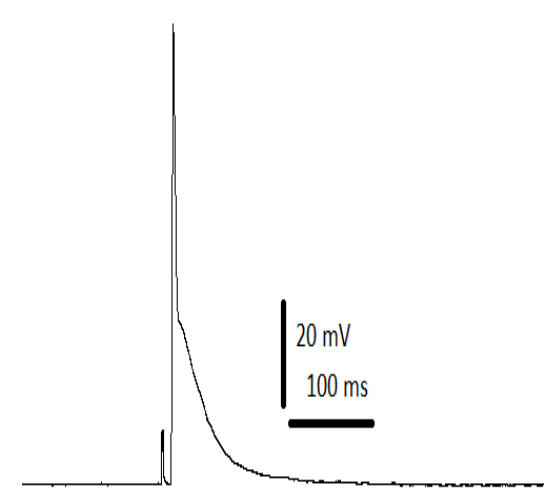

(iii)

Figure 2.7 Synaptic recruitment was observed in MPG neurons in response to pelvic nerve stimulation at increasing stimulus intensities.

(A) Traces showing increase in subthreshold EPSP amplitudes at stimulus intensity (i) $10 \mathrm{~V}$, (ii) $14 \mathrm{~V}$, (iii) $18 \mathrm{~V}$ from the same neuron. Increasing amplitudes of EPSPs can be seen with higher strengths of stimuli. (B) Traces from a neuron that responded with small EPSP at 3V(i), increased EPSP amplitude at $4 \mathrm{~V}$ (ii), and responded with a suprathreshold potential at $5 \mathrm{~V}$ (iii).

\subsubsection{Nicotinic acetylcholine receptor subtypes involved in synaptic transmission}

Our next set of experiments investigated the types of nicotinic acetylcholine receptor (nAChR) subunits involved in this ganglionic transmission. In these experiments, the initial responses to pelvic nerve stimulations were recorded before perfusion of each 
selective antagonists. Then, the effect of the antagonists on the synaptic responses were measured after the maximum blockage has been achieved for each antagonist. No individual cell had been exposed to more than one antagonist.

Pelvic nerve stimulation-induced EPSPs were significantly inhibited (77\% blockage) by $10 \mu \mathrm{M}$ Mecamylamine (non-competitive neuronal $\mathrm{nAChR}$ antagonist) $(\mathrm{n}=11)$, followed by $\alpha 3 \beta 4$ subtype-specific blocker $\alpha$-conotoxin AuIB $(10 \mu \mathrm{M})(75 \%$ blockage $)(n=8)$. Dextromethorphan $(100 \mu \mathrm{M})$ which is supposed to have preferential blockage to $\alpha 3 \beta 4$ nAChR subtype (Damaj et al.,2005) blocked (74\%) (n=7), and Hexamethonium (100 $\mu \mathrm{M})$, a non-specific $\mathrm{nAChR}$ blocker blocked about $63 \%$ of the EPSP amplitudes ( $\mathrm{n}=9)$ (Fig. 7). Dihydro- $\beta$-erythroidine (DH $\beta E$ ) which is supposed to preferentially block $\alpha 3 \beta 4$ subtype at high concentrations $(100 \mu \mathrm{M})$, blocked $52 \%$ of the EPSP amplitudes $(\mathrm{n}=7)$ and blocked $20 \%$ of the EPSPs $(\mathrm{n}=7)$ at $10 \mathrm{uM}$, the concentration that is supposed to preferentially block $\alpha 4 \beta 2$ subtype (Figure. 7) (Harvey \& Luetje, 1996). Atropine(10 $\mu$ M), a muscarinic acetylcholine receptor blocker blocked about (18\%) of the initial EPSP amplitudes ( $\mathrm{n}=8)$ (Figure 2.8).

The blockers not only blocked subthreshold EPSPs but they also reduced action potentials to small-amplitude EPSPs in graded, time-dependent manner. The extent of blockage was different for each blocker. Mecamylamine, $\alpha$-conotoxin AuIB and DHßE $(100 \mu \mathrm{M})$ strongly blocked all the action potentials and reduced them to EPSPs (Figure.8.). Hexamethonium blocked action potentials from 55\% of the cells, Dextromethorphan blocked $80 \%$ of the cells, DHßE $(10 \mu \mathrm{M})$ blocked $40 \%$ of the cells, and Atropine blocked about action potentials from $14 \%$ of the cells (Figure 2.8). 
EPSP amplitudes before and after blocker perfusions
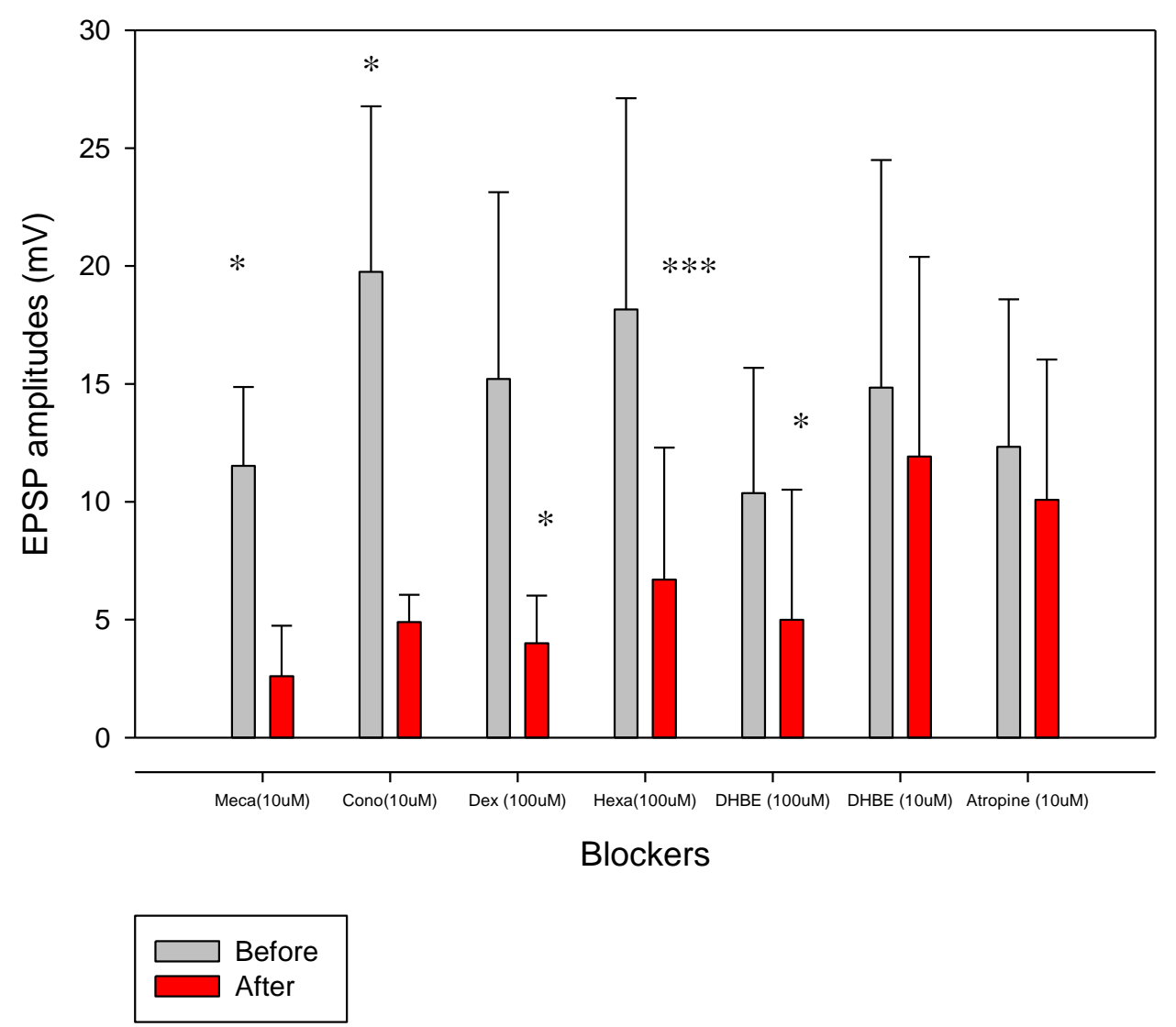

Figure 2.8_Blockage of excitatory postsynaptic potentials (EPSPs) by selective synaptic blockers.

Average amplitudes of EPSPs before and after perfusion of nicotinic acetylcholine receptor antagonists. Data were analyzed by paired t-tests. Mecamylamine $(\mathrm{n}=11) ; P=0.005, \alpha 3 \beta 4$ subtypespecific blocker Conotoxin AuIB ( $\mathrm{n}=8) ; P=0.005$, Dextromethorphan ( $\mathrm{n}=7) ; P=0.001$,

Hexamethonium (n=9); $P<0.001$, Dihydro-beta-erythroidine (100uM) (n=7); $P=0.021$, Dihydrobeta-erythroidine $(10 \mathrm{uM})(\mathrm{n}=7) ; P=0.006$, and muscarinic acetylcholine receptor blocker Atropine $(\mathrm{n}=8) ; P=0.117$. 
Percent of cells with action potentials blocked by blockers

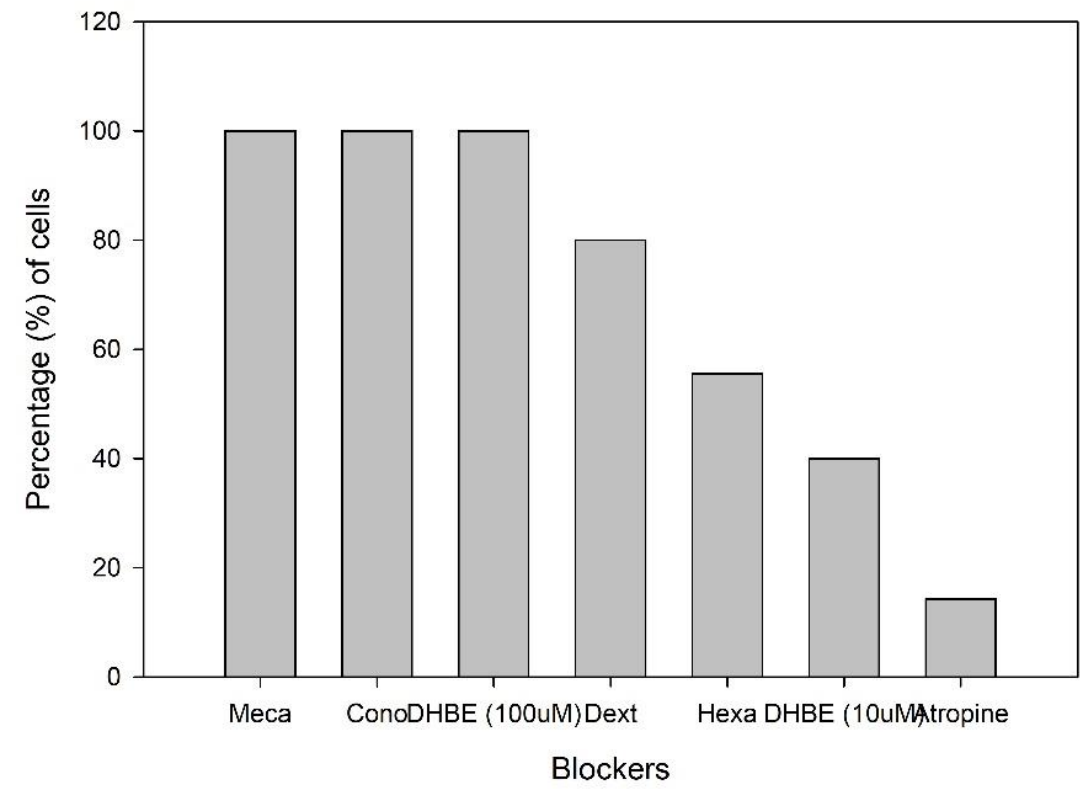

Figure 2.9 Nicotinic acetylcholine receptor antagonists reduced action potentials into subthreshold EPSPs

Mecamylamine, Conotoxin AuIB, DH $\beta E(100 \mu \mathrm{M})$ reduced action potentials from all the neurons to EPSPs. Hexamethonium blocked 55\%, DHßE $(10 \mu \mathrm{M})$ blocked $40 \%$ of the action potentials. Atropine had smallest impact, blocking only $14 \%$ of the action potentials.

\subsubsection{Nicotinic acetylcholine receptor transcript levels}

In addition to pharmacological experiments, we also performed molecular analyses of mRNA transcripts for nAChR subunits $(\alpha 2, \alpha 3, \alpha 4, \alpha 5, \alpha 7, \beta 2$, and $\beta 4)$ using quantitative PCR method. We used GAPDH and $\beta$-actin to standardize the raw copy numbers. The mRNA copy numbers for all the genes were plotted against each other to inspect if any two pairs of $\alpha$ and $\beta$ subunits were expressed in correlated fashion. The results indicated that among all the pairwise correlations, strong correlations were observed between $\alpha 3$ and $\beta 4$ subunits mRNA transcript levels $(\mathrm{R}=0.9688)$. 
(A)

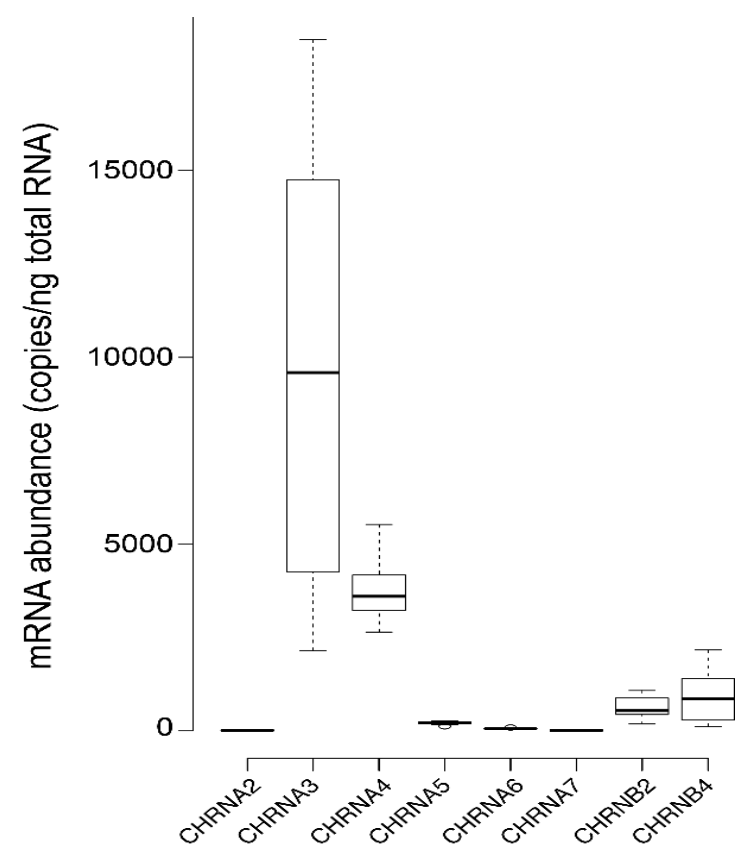

(B)

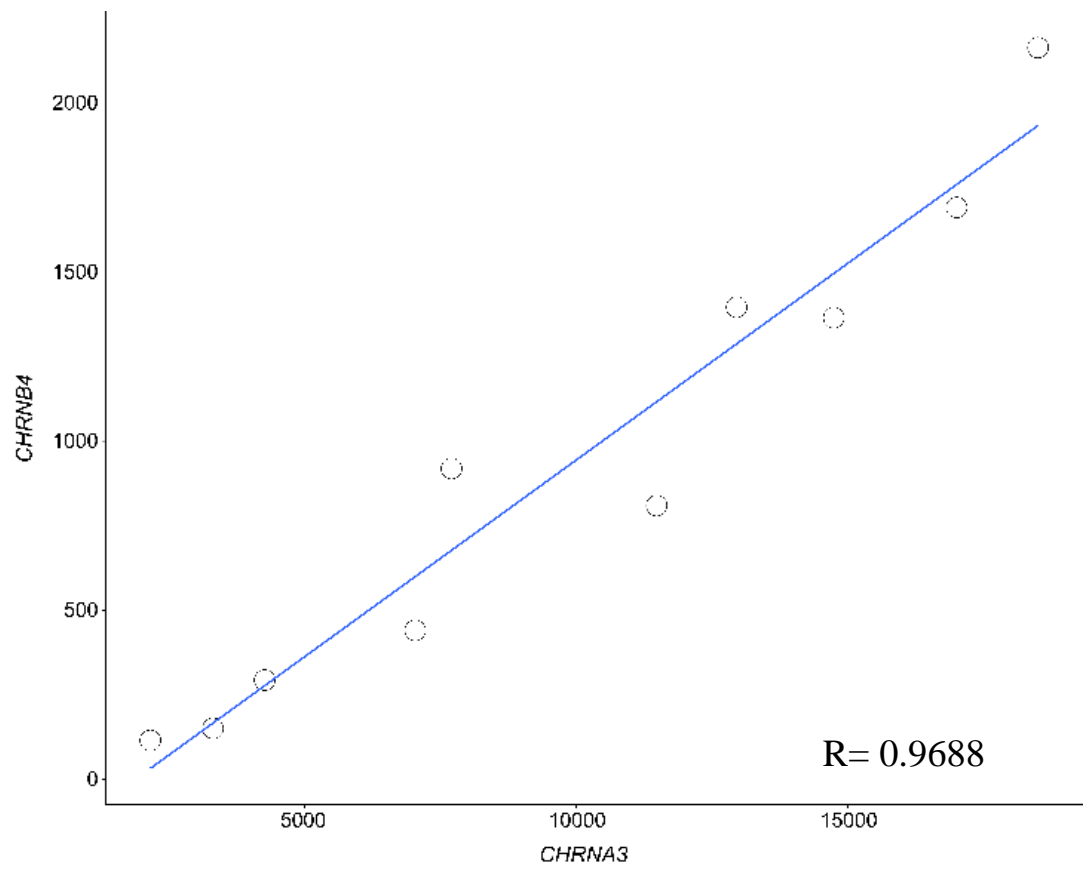

Figure 2.10 (A) Analysis of abundance of mRNA transcripts showed $\alpha 3$ expression to be most abundant among other subunits. (B) $\alpha 3$ and $\beta 4$ subunits showed correlated expression of their mRNA expressions from whole MPGs. 


\subsection{Discussion}

Our study indicates that some of the neurons of mouse major pelvic ganglia (MPG) have capacity for recruiting subthreshold signals to generate action potentials, and there seems to be a range of preferred frequencies of presynaptic inputs that the MPG neurons respond to with high level of reliability. Our study also shows $\alpha 3$ and $\beta 4$ subunits to be the major subunits involved in the ganglionic transmission at the mouse MPG. Most of the cells at the mouse MPG are phasic in nature as they only responded with one action potential to intracellular current injections which is in consistent with the nature of MPG neurons found in rats (Felix et al., 1998) and in female mice (Jobling \& Lim, 2008). The range and mean resting membrane potentials for the MPG neurons are also comparable to those of rats and female mice reported in Felix et al.,1998 and Jobling \& Lim, 2008, respectively.

\subsubsection{Responses of MPG neurons to pelvic nerve stimulation}

In response to pelvic nerve stimulation, $48 \%$ of MPG neurons responded with all or none fast synaptic responses giving rise to action potentials while $52 \%$ responded with excitatory post synaptic potentials (EPSP). This is an unexpected finding since previous studies in female mouse MPG neurons (Jobling \& Lim,2008) reported that MPG neurons generally responded with single suprathreshold potentials. Our results indicated a high percentage of neurons responding with EPSPs suggesting that synaptic transmission at the MPG does not always follow one-to-one signal transmission with the presynaptic inputs, and hence the potential for facilitation or modulation of synaptic transmission exists. 
Whether a neuron responded with all-or-none suprathreshold action potentials or subthreshold EPSPs did not depend on the membrane potential of the neurons. Our finding of more than $50 \%$ of the neurons tested responded with subthreshold EPSPs is higher than those reported for rat MPG (Felix et al., 1998), in mouse hypogastric ganglia (Rogers et al.,1990), and in female mouse MPG (Jobling \& Lim, 2008). Various shapes of fast-synaptic potentials observed are also similar to those found in mouse hypogastric ganglion (Rogers et al.,1990). The amplitudes of large EPSPs ( 20 to $40 \mathrm{mV})$ were comparable to those reported for rat MPG neurons by Tabatabai et al., 1986. The nature of synaptic responses in which large suprathreshold EPSPs giving rise to action potentials in some of the neurons are also similar to those in rat MPG neurons (Tabatabai et al.,1986; Felix et al., 1998).

\subsubsection{Membrane properties and action potential properties of MPG neurons}

The only significant differences in membrane properties of those neurons that responded with all-or-none action potentials and those with EPSPs were for apparent input resistance and apparent capacitance. The EPSP-generating neurons seem to have larger capacitances than action potential-generating neurons. All other properties were not significantly different. Action-potential generating neurons seem to have higher apparent input resistance and lower apparent capacitance than those neurons that responded with subthreshold potentials. Having higher input resistance could be one of the reasons for generating suprathrehold potentials. Another likely explanation could be that that those neurons with action potentials had large amount of neurotransmitter release from the presynaptic bouton for a single stimulus or had synapses on spike initiation zones where the threshold for action potential generation is relatively lower. Another likely factor is 
that the neurons that responded with subthreshold potentials had higher threshold to fire action potentials. We could only speculate the mechanisms since we did not perform experiments to examine the underlying mechanisms.

We did not find significant differences between male and females MPG neurons in either membrane properties or in the properties of rebound action potentials. However, significant differences were found between the action potential-generating cells and EPSP-generating cells in the rebound action potential properties. The action potentials from those neurons that responded with subthreshold EPSPs had more depolarized resting potentials, shorter peak amplitudes, wider half-widths, shorter AHPs with slower rise slopes and decay slopes. These differences could be due to the differences in the nature of voltage-gated ion channels present and kinetics of ionic currents present in these MPG neurons. Future studies with voltage-clamp experiments would need to be carried out to explore the mechanisms underlying the nature of these two groups of neurons.

We cannot rule out the methodological impacts such as quality of cell impalement which could contribute to any of these results. Sharp electrode properties may change basic properties of these cells, such as leak currents (Springer et al., 2015), and influence the interpretation of these results. However, we tried to accommodate for these issues with robust sample size to add as much power to the analysis as possible. In addition, we would expect that neurons that respond with subthreshold EPSPs may do so due to a more negative resting membrane potential relative to those that generated action potentials, and lower input resistance. This was not the case. EPSP generating cells were 
more depolarized at rest, potentially being closer to the threshold. Therefore, our data are not consistent with an interpretation solely on differences in impalement across neurons.

\subsubsection{Frequency-dependent modulation of synaptic transmission at the MPG neurons}

Increasing degrees of depression of synaptic responses were observed in $75 \%$ of the cells tested as the frequency of pelvic nerve stimulation was increased from $2 \mathrm{~Hz}$ to $20 \mathrm{~Hz}$. These results are similar to those of guinea pig and male rat MPGs which showed depression of synaptic responses during repetitive nerve stimulation (Blackman et al., 1969; Tabatabai et al., 1986; Felix et al., 1998) but opposed to those in cat MPG neurons in which significant facilitation of synaptic responses were observed at high frequencies of nerve stimulation, and are reported to act as high-pass filters (de Groat \& Saum, 1976). Increasing frequency of nerve stimulation not only reduced the amplitudes of EPSPs, it also reduced the reliability of firing of all-or-none action potentials which is in consistent with those reported for rat MPG neurons (Tabatabai et al., 1986; Felix et al., 1998). Our findings of decreased EPSP amplitudes and reliability of action potential firings at higher frequencies $(10 \mathrm{~Hz}-20 \mathrm{~Hz})$ indicate that the mouse MPG may act as a low-pass filter (Abbott \& Regehr 2004) and that there is a rate-limiting factor of synaptic transmission at this ganglion. We did not explore what is limiting the reliable signal transmission at higher presynaptic input frequencies. However, we can speculate that this could be due to depletion or lower turnover of readily-releasable pool of transmitter vesicles in the presynaptic boutons at higher frequencies of activation (Dobrunz, 2002; Neher \& Sakaba, 2008; Fortune \& Rose, 2001; Bruckner et al., 2016) , or due to desensitization of nicotinic acetylcholine receptors on the postsynaptic membrane (Chen et al.,1999; Kim et al., 2015; Galarreta \& Hestrin, 1998; Kottick \& Del Negro 2015; Okatan \& Grossberg, 
2000; Miles, 1986). These findings show that the input signals at mouse MPG neurons could be modulated depending on frequency, and the signals will likely fail to be transmitted at higher frequencies indicating rate-limiting properties of MPG synapses.

\subsubsection{Spatial summation at the MPG synapses}

Our data showed that 53\% of neurons tested showed summation of responses at higher strengths of pelvic nerve stimulation. We found graded increase of small EPSPs to large EPSPs (in 69\% of neurons tested) as well as increase from EPSPs to action potentials with little gradation (in $31 \%$ of neurons tested). This is in oppose to those reported for EPSPs generated in mouse hypogastric ganglia (Rogers et al., 1990) where the EPSPs were evoked in all-or-nothing manner. The graded nature of EPSPs in our findings suggests that some mouse MPG neurons have more than one synaptic input and have the potential for summation of inputs. Unlike those reported for female paracervical ganglia neurons (Jobling \& Lim, 2008), and for rat MPG neurons (Felix et al., 1998), not all the neurons respond with all-or-none suprathreshold action potentials in our experiments. These findings imply that the synaptic transmission at MPG neurons has the potential for summation according to the intensity and frequency of the neuronal signals as well as based on the morphology of the synapses. This increase in amplitude at increasing strengths of stimulation shows that some of the mouse MPG neurons are capable of summing the inputs from multiple sources and elicit a combined synaptic output.

\subsection{5 mRNA expression of nicotinic acetylcholine receptor (nAChR) subunits}

Neuronal nicotinic acetylcholine receptors (nAChRs) play a key role in the control of bladder function as mediators of both parasympathetic and sympathetic fast synaptic transmission at MPG (DeGroat and Saum, 1976; Gallagher et al., 1982; Biasi, 2000; Park 
et.al, 2006). The nicotinic cholinergic system consists of different nAChR subunits assembled in various fashions to form different receptor phenotypes. Receptor phenotypes differ from each other in terms of specific ion permeability, ion conductance, and changes in synaptic transmission due to various $\mathrm{Ca}^{2+}$ permeabilities (Skok, 2002). Our pharmacological study suggests that $\alpha 3 \beta 4$ subtype is the major subtype involved in MPG synaptic transmission as indicated by major blockage by Conotoxin AuIB. Minor contributions from $\alpha 4 \beta 2$ is a possibility as evidenced by minor blockages by $\mathrm{DH} \beta \mathrm{E}$ $(10 \mu \mathrm{M})$. This finding is consistent with those found in other autonomic ganglia (DeGroat and Saum, 1976; Gallagher et al., 1982; Biasi, 2000; Park et.al, 2006; Skok, 2002; De Biasi, 2002). Blockage by Atropine, although not significant, suggests the minor role of muscarinic AChRs at the synaptic transmission at the MPG neurons. The presence of muscarinic AChRs, even though very minor, is similar to the findings in the mouse hypogastric ganglia (Rogers et al., 1990). The blockers not only blocked EPSPs but they also reduced action potentials into EPSPs in graded, time-dependent manner. The fact that the blockers did not block both action potentials and EPSPs in all-or-none manner suggest that the MPG neurons could compose of more than one type of nicotinic receptor subtype with various levels of involvement in the synaptic transmission.

Our molecular analyses showed $\alpha 3$ subunit to be the most abundant subunit among other nAChRs suggesting $\alpha 3$ to be a major subunit in cholinergic transmission at the MPG. Interestingly, we also found correlated mRNA expression between $\alpha 3$ and $\beta 4$ subunits, suggesting that these two subunits could form a functional nAChR subtype in neurons of the mouse MPG. These results are consistent with our physiology data using subtypespecific blockers which showed the highest blockage for $\alpha 3 \beta 4$ subtype. These data are 
also consistent with the literature since $\alpha 3$ subunit is found to be a predominant subunit in the autonomic ganglia and thought to form heteromeric $\mathrm{nAChRs}$ with other $\alpha$ and $\beta$ subunits (Skok, 2002; Wang, 2002). $\alpha 3$ subunit mediates the monovalent cation and $\mathrm{Ca}^{2+}$ conductance increases in response to acetylcholine (ACh) and nicotinic agonists (Skok, 2002). Hence, our data suggests that $\alpha 3$ and $\beta 4$ can be considered major functional nAChR subunits at the MPG neurons.

Our findings indicate that in consistent with literature from other species, most of the MPG neurons are phasic. We observed higher percentage of cells that responded with subthreshold EPSPs compared to those reported in literature, and also observed differences between action potential properties of MPG neurons that responded with suprathrehold action potentials and those that responded with subthreshold EPSPs. MPG neurons seem to act as low-pass filters as shown by frequency-dependent depressions of EPSPs as well as by decrease in reliability of generating action potentials at higher presynaptic nerve stimulated frequencies. In consistent with the literature, both our pharmacology and mRNA experiments suggest that $\alpha 3$ and $\beta 4$ are major $n A C h R$ subunits present in mouse MPG neurons. We did not find significant differences in passive and action potential properties of male and female MPG neurons.

Understanding of cholinergic efferent neurotransmission at the ganglionic level in normal functional state is important for a complete understanding of neural mechanisms involved in control of urogenital organs. Literature suggests species differences in the role of major pelvic ganglia in neural control of pelvic organs. Hence, it is important to closely examine the nature of synaptic transmission in the species of interest as well as in both sexes due to differences in the nature of target organs that the MPG neurons innervate. In 
this study, we assumed that the neurons that responded to pelvic nerve stimulations to be parasympathetic neurons. However, we did not identify the MPG neurons based on the target organ that they innervate. Future experiments should explore both presynaptic and postsynaptic mechanisms underlying the frequency-dependence of responses in MPG neurons, the synaptic and ionic mechanisms underlying the properties of those neurons that respond with all-or-none action potentials versus subthreshold EPSPs in identified MPG neurons of specific sex. Understanding the nature of synaptic transmission at the major pelvic ganglia and contributions of nAChR subunits involved in this neurotransmission is important to understand how the MPG neurons are affected in cases of disease or nerve injuries that disrupt the autonomic functions of pelvic organs innervated by the major pelvic ganglia. 


\section{CHAPTER 3 \\ EFFECTS OF DECENTRALIZATION ON CHOLINERGIC NEUROTRANSMISSION AT THE NEURONS OF MOUSE MAJOR PELVIC GANGLIA}

\subsection{Introduction}

Major pelvic ganglia (MPG) of mouse contains postganglionic neurons innervating urinary bladder, the genitals and distal colon (Purinton, 1972; Keast, 1995). Pelvic ganglia receive cholinergic parasympathetic inputs from the preganglionic neurons in the sacral cord through pelvic nerve, and sympathetic inputs from those in the lumbosacral cord through hypogastric nerve (Kihara \& de Groat 1997; Keast, 1995). The axons from the pelvic ganglion synapse onto postganglionic neurons on the target organ, releasing acetylcholine from parasympathetic axons and noradrenaline from sympathetic axons (Dail et al., 1975; de Groat \& Saum, 1976; Papka et al., 1999; de Groat \& Yoshimura, 2015).

Damage to the pelvic nerve due to complications from surgeries carried out in the pelvic area often results in micturition and erectile dysfunctions (Nangle \& Keast, 2009; Girard et al., 2013; Delacroix Jr. \& Winters, 2010). Pelvic nerve damage also causes urinary complications such as abnormalities in urinary storage, poorly compliant bladder, and is a common consequence of surgical procedures done in the pelvic area (Girard et al., 2013; Delacroix \& Winters, 2010). Urinary incontinence due to pelvic nerve damage is one of the major complications from surgical procedures in the pelvic area for bladder, prostate and colorectal cancers (Nangle \& Keast, 2009; Girard et al., 2013; Delacroix \& Winters, 2010). Initial symptoms of failure to empty and abnormalities in urinary storage may lead 
to small capacity, and poorly compliant bladders. In some patients, normal bladder function may not be regained, and patients may persist with noncompliant bladders and upper urinary tract dysfunction (Delacroix \& Winters, 2010).

Neuronal nicotinic acetylcholine receptors (nAChRs) play a key role as mediators of both parasympathetic and sympathetic fast synaptic transmission at the MPG (DeGroat and Saum, 1976; Gallagher et al., 1982; Biasi, 2000; Park et.al, 2006). nAChRs are ligandgated ion channels composed of five subunits to form a homopentamer or heteropentamer (Figure 4) (Albuquerque et al.,1996; Skok, 2002; Park.et.al., 2006). Twelve genes have been identified to encode neuronal nAChR subunits: $\alpha(2-10)$ and $\beta(2-4)$ subunits (Biasi, 2002). The main subunits found in autonomic ganglia are $\alpha 3, \alpha 4, \alpha 5, \alpha 7, \beta 3$, and $\beta 4$ (Skok, 2002). $\alpha 2$ - $\alpha 4$ subunits can form functional channels only if co-expressed with a $\beta 2$ or $\beta 4$ subunit (Skok, 2002; Park et al, 2006).

It was shown that cavernous nerve injury caused downregulation of $\mathrm{nAChR}$ subunit expression and interruption of synaptic transmission within mouse MPG and subsequent contribution to loss of neural control of urogenital organs after pelvic surgeries (Girard et al., 2013). It was also shown that pelvic nerve injury in rats caused changes in spinal nAChR subunit compositions (Young et al., 2008). These findings in the literature indicate changes upstream of the injury site in response to nerve injury or loss of input.

However, the effects of disruptions in preganglionic inputs on MPG neurons have not been studied. The goal of this project is to understand the effect decentralization has on electrical and synaptic properties as well as on nicotinic cholinergic transmission at the major pelvic ganglia at acute and chronic stages. Understanding how the MPG neurons 
response to decentralization will give us a baseline to understand the ganglionic response to altered inputs in cases of nerve injuries as well as spinal cord injury. It is important to understand the plasticity of MPG neurons in these conditions as the MPG sends neural signals directly to the target organs and can affect organ functions.

Our hypothesis is that in response to loss of synaptic inputs, synaptic changes such as postsynaptic nAChR reorganization will occur in order to compensate for the loss of input. These changes in synaptic transmission will, in turn, cause changes in membrane properties of the MPG neurons.

We performed unilateral decentralizations by transecting pelvic and hypogastric nerves on the right side of the mouse, about $2 \mathrm{~mm}$ before their point of entry to the ganglion. The left side was left intact. We employed molecular and electrophysiology techniques on sham-surgical control and decentralized adult male Swiss Webster mice. We measured the mRNA abundance of $\alpha 2, \alpha 3, \alpha 4, \alpha 5, \alpha 6, \alpha 7, \beta 2$ and $\beta 4$ neuronal nicotinic receptor subunits in whole MPGs using quantitative PCR (qPCR). We then measured electrical properties and synaptic transmission at MPG neurons using both current and voltage clamp methods. We measured synaptic strengths by measuring excitatory postsynaptic currents (EPSCs) in response to pressure ejections of acetylcholine (ACh).

\subsection{Materials and Methods}

\subsubsection{Animal numbers}

Adult male Swiss-Webster mice $(\sim 30 \mathrm{~g})$ were used $(\mathrm{n}=26)$. Animals were grouped into two experimental groups- acute injury (24-hour post-injury), chronic injury (28 days post-injury) groups and two respective sham-control groups. The 24-hour post-injury 
period is chosen for acute injury because significant decrease in $\mathrm{nAChR}$ expression levels were observed in autonomic ganglia after peripheral nerve injury studies (McCann et al., 2008; Peddie \& Keast, 2011; Girard et al., 2013). The recovery of nAChR levels and synaptic transmission were observed to be slowly recovering around the 3-week period and hence 1-month post-injury time point seems reasonable for chronic injury stage (McCann et al., 2008; Peddie \&Keast, 2011; Girard et al., 2013). All experimental procedures were approved by the University of Missouri Institutional Animal Care and Use Committee and conformed to the National Institutes of Health Guide for the Care and Use of Laboratory Animals.

\subsubsection{Preparing the animals and induction of anesthesia}

Mice were given $5 \mathrm{mg} / \mathrm{kg}$ carprofen through subcutaneous injection before getting anesthetized using isoflurane $(0.5 \mathrm{ml})$ in a chamber. After the induction of anesthesia, anesthesia was maintained by inhalation of isoflurane through a nose-cone. To ensure proper depth of anesthesia, mice were checked for reflexes by pinching the toes.

Afterwards, the pelvic area of each animal was shaved. The area was then cleaned with betadine swaps, leaving the contact time of 5 minutes. Then, betadine from the area was wiped with alcohol swap.

\subsubsection{Surgery}

An incision of about $1.5 \mathrm{~cm}$ is made with the sharp end of a blade to cut through the skin and the muscle layers. The bladder is located and fat tissues around the bladder neck are removed with care not to cut any small blood vessels. Major pelvic ganglion (MPG) which lies on the lateral side of prostate tissue is located. MPG receives inputs from the 
pelvic nerve, hypogastric nerve and supplies the organs of the lower urinary tract and genital organs. The pelvic nerve was located along the dorsolateral side of the prostate gland, and the nerve is transected about $2 \mathrm{~mm}$ away from its entry to the ganglia. The hypogastric nerve was also transected in order to carry out complete decentralization of inputs to the MPG. Then, the bladder and surrounding organs were put back into the pelvic cavity. The muscle layer was sutured, and the skin was closed by using skin staples.

\subsubsection{Tissue isolation for in-vitro recordings}

MPGs were dissected out from the animals after euthanizing with overdose of isoflurane inhalation. The MPGs were dissected out and pinned down in a sylgard dish with oxygenated HEPES buffer solution containing, (in $\mathrm{mM}$ ) $\mathrm{NaCl}, 146 ; \mathrm{KCl}, 4.7 ; \mathrm{MgSO}_{4}$, 0.6; $\mathrm{NaHCO}_{3}, 1.6 ; \mathrm{NaH}_{2} \mathrm{PO}_{4}, 0.13 ; \mathrm{CaCl}_{2}, 2.5 ;$ Glucose, 7.8; HEPES, 20), adjusted to $\mathrm{pH}$ 7.3 (Jobling and Lim, 2008). The saline was preheated to $35^{\circ} \mathrm{C}$ and aerated with oxygen before being perfused into recording chamber.

\subsubsection{In-vitro electrophysiology recordings}

The dissected MPGs were de-sheathed and cleaned off from connective tissue and fat. The ganglia were pinned down on a piece of Sylgard glued in a $1 \mathrm{ml}$ recording chamber. Intracellular recordings were made by impaling individual MPG neurons with sharp glass electrodes. The recording electrodes contain intracellular solution of $0.5 \mathrm{M} \mathrm{KCl}$ solution and has resistances in the range between $60 \mathrm{M} \Omega-80 \mathrm{M} \Omega$. AxoClamp $900 \mathrm{~A}$ (Molecular Devices) amplifier was used for clamping voltages and recording the signals and Digidata 1440A digitizer was used to digitize the signals at $10 \mathrm{kHz}$. The resting membrane 
potential and voltage responses to acetylcholine $(\mathrm{ACh})$ pressure ejection puffs were measured in Bridge mode. Current responses were measured in dSEVC (Discontinuous single-electrode voltage clamp) mode by clamping each neuron under study at its resting membrane potential and at the sampling frequency of $3 \mathrm{kHz}-5 \mathrm{kHz}$, with gains ranging from $0.5 \mathrm{nA} / \mathrm{mV}$ to $3 \mathrm{nA} / \mathrm{mV}$. The signals were acquired in Clampex 10.3 software and data was analyzed in Clampfit 10.3 program.

\subsubsection{Acetylcholine pressure ejections}

Picospritzer (General Valve Corporation, Picospritzer II) was used for pressure ejection of acetylcholine. A glass electrode with resistances ranging from $2 \mathrm{M} \Omega$ to $4 \mathrm{M} \Omega$ was filled with acetylcholine $1 \mathrm{mM}$ (Acros Organics) and the pressure was set around $10 \mathrm{psi}$. Duration for each pressure puff is $0.5 \mathrm{~s}$. Care was taken to ensure consistent distances from tip of the pressure ejection electrode to the cell under study. Pressure ejection electrode was placed about 50-75 $\mu \mathrm{m}$ away from each cell under study.

\subsubsection{Excitability test}

Excitability of the neurons were measured by injecting depolarizing and hyperpolarizing current pulses of $400 \mathrm{pA}$ with durations around $300 \mathrm{~ms}$ in bridge mode. The firing patterns of action potentials in response to current injections were observed as tonic or phasic neurons.

\subsubsection{Quantitative PCR (qPCR)}

Total RNA was isolated from MPGs using Trizol according to the protocol provided by the manufacturer (Invitrogen). cDNA was generated from $100 \mathrm{ng}$ total RNA primed with 
a mixture of oligo-dT and random hexamers that was reverse transcribed using qScript reverse transcriptase (QuantaBio). The final volume of the reverse transcription reaction was $20 \mu \mathrm{l}$,and contained a final concentration of $2.5 \mathrm{ng} / \mu 1$ random hexamers, $2.5 \mu \mathrm{M}$ oligo-dT, $40 \mathrm{U}$ of RNase inhibitor, and $200 \mathrm{U}$ of reverse transcriptase. Following heat inactivation of the enzyme, samples were diluted in ultrapure water to a final volume of $175 \mu \mathrm{l}$ before this template was used in qPCR analyses. From each cDNA pool generated from $100 \mathrm{ng}$ of total RNA, we were quantified at least 15 different gene products. We designed or modified, and independently validated primer sets for use in absolute quantitation of copy number for (8) distinct genes of interest from the mouse (see Table 1). $\mathrm{qPCR}$ reactions consisted of primer pairs at a final concentration of $2.5 \mu \mathrm{M}$, cDNA template, and SsoAdvanced SYBR mastermix (BioRad) according to the manufacturer's instructions. Reactions were carried out on a CFXConnect (BioRad) machine with a three-step cycle of $95^{\circ} \mathrm{C}-15 \mathrm{~s}, 58^{\circ} \mathrm{C}-20 \mathrm{~s}, 72^{\circ} \mathrm{C}-20 \mathrm{~s}$, followed by a melt curve ramp from $65^{\circ} \mathrm{C}$ to $95^{\circ} \mathrm{C}$. Data were acquired during the $72^{\circ} \mathrm{C}$ step, and every $0.5^{\circ} \mathrm{C}$ of the melt curve. All reactions were run in triplicate, and the average $\mathrm{Ct}$ (cycle threshold) was used for interpolation with the standard curve to generate copy number for a given reaction. Standard curves for each gene were generated from a known copy number of a plasmid containing a partial ORF for a given gene of interest. Plasmids of known copy number were diluted from 106 copies $/ \mu 1$ to 10 copies $/ \mu$ by factors of $10 \mathrm{x}$, and run in identical qPCR reactions to the samples. A line was fit to the standard curve and the subsequent equation for the fit line used to interpolate copy number from $\mathrm{Ct}$ values obtained from biological samples. 
The units with which we express all of the qPCR data in this study is "normalized copy number per ng total RNA." All of the data are normalized relative to Glyceraldehyde 3phosphate dehydrogenase (GAPDH) expression. Briefly, each sample is normalized to the population mean for each gene of interest by creating an adjustment factor based on GAPDH expression above or below the population average. A normalization factor using GAPDH for a sample $\mathrm{x}$ was calculated by the following formula:

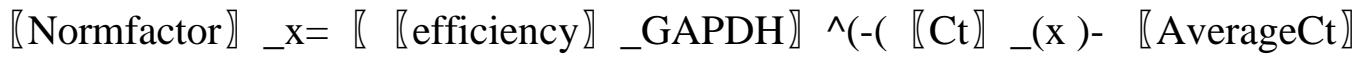
_GAPDH))

For our assay, GAPDH amplification efficiency was virtually $100 \%$, so this can be simplified as:

\section{『Normfactor】_x $=2^{\wedge}\left(-\llbracket \Delta \mathrm{Ct} \rrbracket \_\right.$GAPDH $)$}

where $\llbracket \Delta \mathrm{Ct} \rrbracket$ GAPDH is the difference between the sample GAPDH and the population average, and 2 is the base of the exponential since $100 \%$ efficiency results in a doubling of product in each cycle of PCR. This resulted in the normalized copy number for a sample $\mathrm{x}$ calculated as:

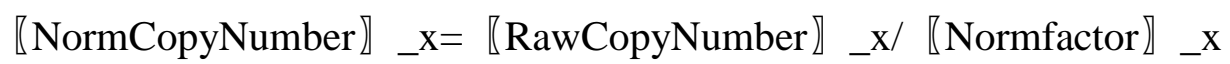

This normalization factor allows us to preserve the overall magnitude of the raw copy number, allowing for comparisons of mRNA abundance across genes. In addition, the normalization adjusts the copy number across different slices to account for differences in mRNA abundance that may occur for technical reasons, including variability in RNA extraction and reverse transcription efficiencies. 


\begin{tabular}{|c|c|c|c|c|}
\hline Gene name & Accession \# & Gene Function & $\begin{array}{l}\text { Forward primer (5' to } \\
\text { 3') }\end{array}$ & Reverse Primer (5' to 3') \\
\hline CHRNA2 & NM_144803 & Nicotinic $\alpha 2$ & $\begin{array}{l}\text { TTATCTCTGGTGTC } \\
\text { TGCTTCTGA }\end{array}$ & $\begin{array}{l}\text { CCCAGCGATTGTAGCCT } \\
\text { CC }\end{array}$ \\
\hline CHRNA3 & NM_145129 & Nicotinic $\alpha 3$ & $\begin{array}{l}\text { TCCAGTTTGAGGTG } \\
\text { TCTATGTCT }\end{array}$ & $\begin{array}{l}\text { TGGTAGTCAGAGGGTT } \\
\text { TCCATTT }\end{array}$ \\
\hline CHRNA4 & NM_015730 & Nicotinic $\alpha 4$ & $\begin{array}{l}\text { CTAGCAGCCACATA } \\
\text { GAGACCC }\end{array}$ & $\begin{array}{l}\text { GACAAGCCAAAGCGGA } \\
\text { CAAG }\end{array}$ \\
\hline CHRNA5 & NM_176844 & Nicotinic $\alpha 5$ & $\begin{array}{l}\text { ATCCTCTGCTGCAA } \\
\text { AACATGA }\end{array}$ & $\begin{array}{l}\text { TCCACGTCCACTAACTG } \\
\text { AGAT }\end{array}$ \\
\hline CHRNA6 & NM_021369 & Nicotinic $\alpha 6$ & $\begin{array}{l}\text { TAAAGGCAGTACA } \\
\text { GGCTGTGA }\end{array}$ & $\begin{array}{l}\text { AAAATGCACCGTGACG } \\
\text { GGAT }\end{array}$ \\
\hline CHRNA7 & NM_007390 & Nicotinic $\alpha 7$ & $\begin{array}{l}\text { CACATTCCACACCA } \\
\text { ACGTCTT }\end{array}$ & $\begin{array}{l}\text { AAAAGGGAACCAGCGT } \\
\text { ACATC }\end{array}$ \\
\hline CHRNB2 & $\begin{array}{l}\text { NM_009602. } \\
4\end{array}$ & Nicotinic $\beta 2$ & $\begin{array}{l}\text { ATTATCGCCTCACA } \\
\text { TGGAAGC }\end{array}$ & $\begin{array}{l}\text { TGCCGTCAGCATTGTTG } \\
\text { TATAG }\end{array}$ \\
\hline CHRNB4 & $\begin{array}{l}\text { NM_148944. } \\
4\end{array}$ & Nicotinic $\beta 4$ & $\begin{array}{l}\text { GGCAAGATCTACA } \\
\text { GGAAGCAT }\end{array}$ & $\begin{array}{l}\text { ACACAATCACGAACAC } \\
\text { CCA }\end{array}$ \\
\hline$A C T B$ & NM_007393 & Beta-actin & $\begin{array}{l}\text { GATGACCCAGATCA } \\
\text { TGTTTGAGACC }\end{array}$ & $\begin{array}{l}\text { AGATGGGCACAGTGTG } \\
\text { GGTGA }\end{array}$ \\
\hline GAPDH & NM_008084 & $\begin{array}{l}\text { Glyceraldehyde } \\
\text { 3-phosphate } \\
\text { dehydrogenase }\end{array}$ & $\begin{array}{l}\text { TGCACCACCACCTG } \\
\text { CTTAGC }\end{array}$ & $\begin{array}{l}\text { GGCATGGACTGTGGTC } \\
\text { ATGAG }\end{array}$ \\
\hline
\end{tabular}

Table 3.1 Gene identification and primer sets used for real-time PCR reactions.

Accession numbers provided for each gene of interest. Primer sets were obtained from PrimerBank or generated de novo using PRIMER3 software. All primers sets were validated before use.

\subsection{9 $\quad \underline{\text { Statistics }}$}

Data were analyzed using Sigmaplot 11.0 using paired t-tests, Mann-Whitney Rank Sum tests, and Pearson correlation test as appropriate. The data are presented as mean+S.E. 


\subsection{Results}

Synaptic transmission of MPG neurons was measured by applying $0.5 \mathrm{~s}$ acetylcholine (1 $\mathrm{mM}$ ) pressure ejections onto individual neurons while voltage clamping the neurons and measuring the inward currents in response to acetylcholine. Data between sham and decentralized groups for corresponding sides at each time point were analyzed using Mann-Whitney Rank Sum test or unpaired t-tests as appropriate. Significant increases in current amplitudes were observed in decentralized groups compared to sham groups in neurons from both ipsilateral and contralateral ganglia at both 24-hour and 28-day groups (Fig 3.1A). $24 \mathrm{hr}$ ipsilateral group had median current amplitudes of $0.526 \mathrm{nA}$ for sham $(n=15)$ and $0.726 \mathrm{nA}$ for decentralized $(n=20)$ groups with significant differences $(\mathrm{P}=0.021)$ (Fig 3.1A). Contralateral side from decentralized MPGs also had highly significant increase $(\mathrm{P}=0.003)$ in response to acetylcholine with median current amplitudes of $1.142 \mathrm{nA}(\mathrm{n}=21)$ compared to median amplitude of sham group $(\mathrm{n}=16)$ which was $0.367 \mathrm{nA}$ (Fig 3.1A).

Chronically decentralized groups on ipsilateral side $(n=12)$ also had increased median current amplitudes of $0.768 \mathrm{nA}$ compared to the sham $(n=13)$ counterpart with median amplitude of $0.468 \mathrm{nA}(\mathrm{P}=0.034)$ (Fig 3.1A). The contralateral side in 28-day sham $(n=13)$ and decentralized $(n=23)$ groups did not have statistically significant differences $(\mathrm{P}=0.331)($ Fig 3.1A). 
(A)

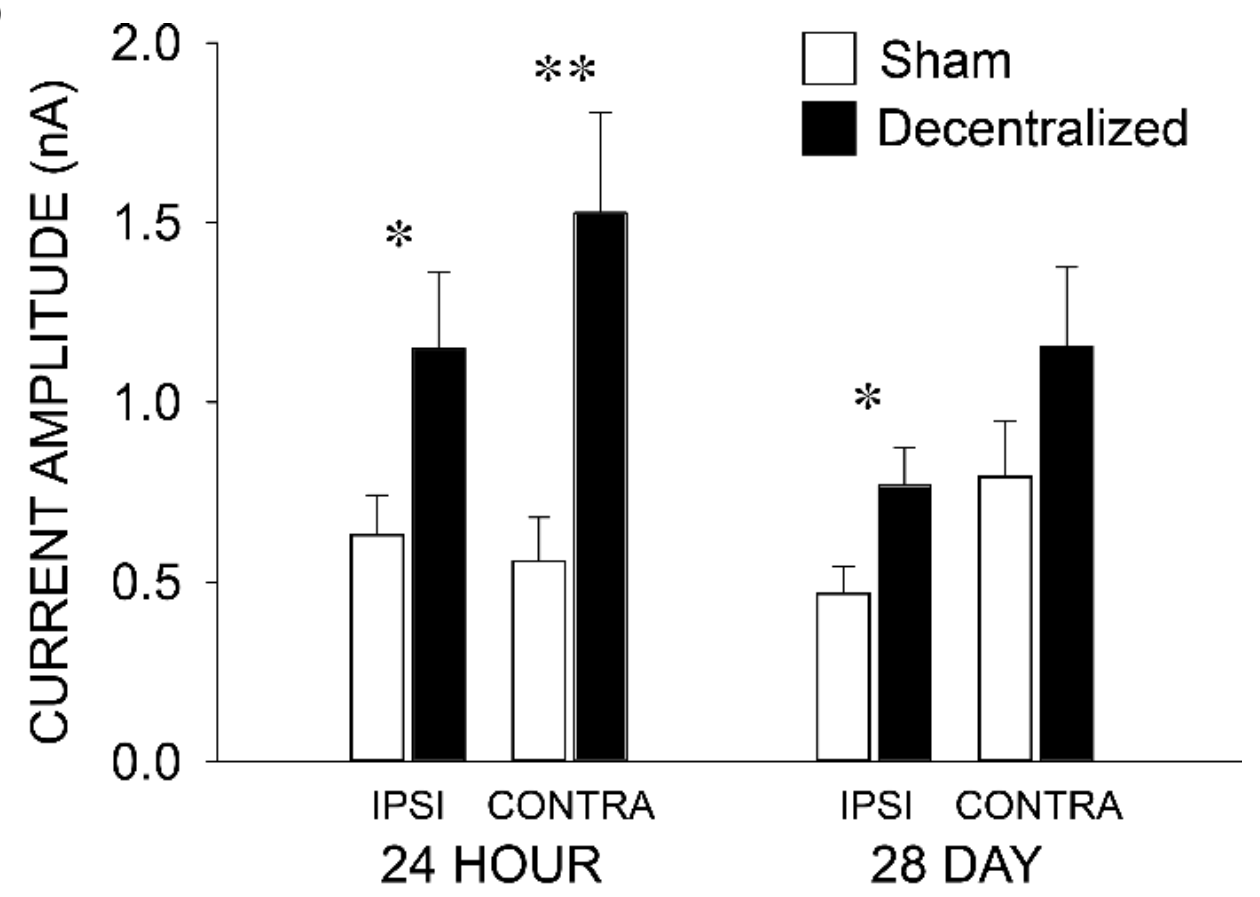

(B)

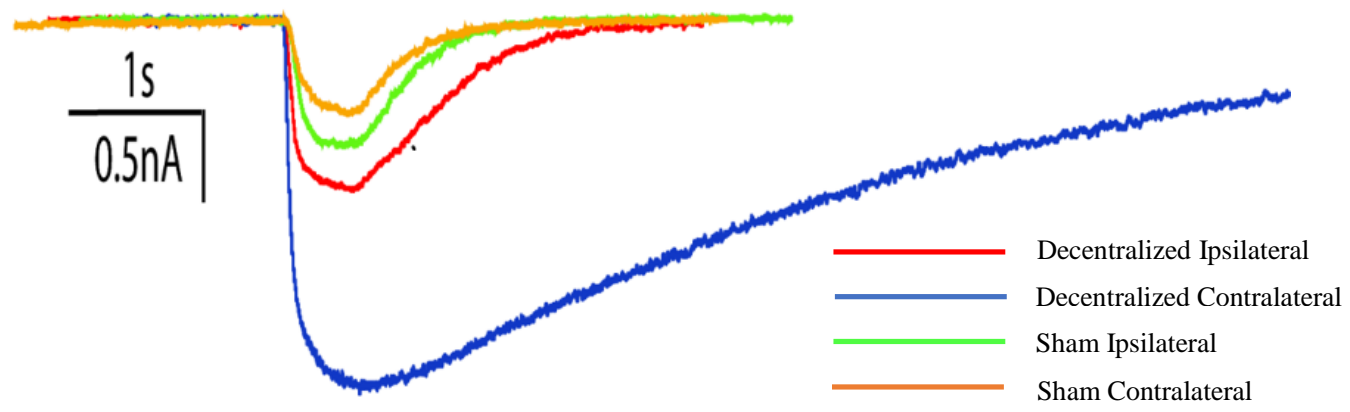

Figure 3.1 MPG neurons from decentralized ganglia show increased current amplitudes in response to acetylcholine pressure ejections.

Contralateral sides from decentralized groups show higher increase from their sham counterparts compared to ipsilateral decentralized groups. A. Current amplitudes in response to acetylcholine in sham groups compared to corresponding sides in decentralized groups (mean \pm S.E). Data are analyzed using Mann-Whitney Rank Sum tests. * indicates $\mathrm{P}<0.05$ and $* *$ indicates $\mathrm{P}<0.005$. B. shows representative current traces in response to acetylcholine pressure ejections from 24-hour groups. 


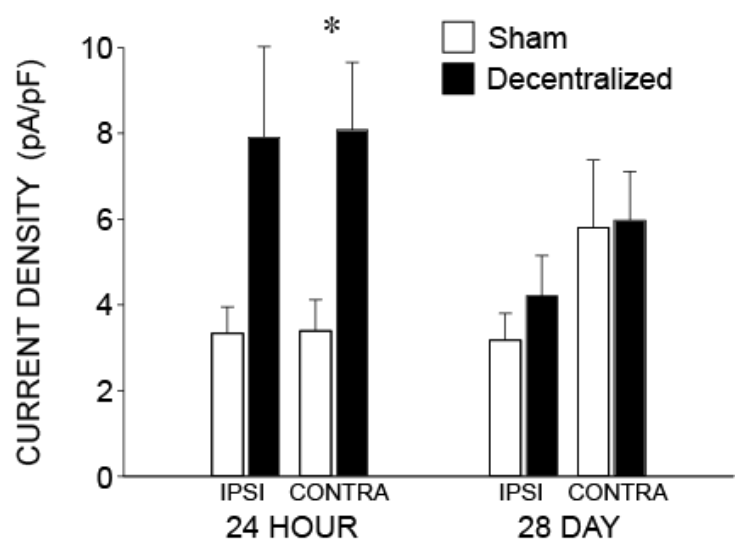

(A)

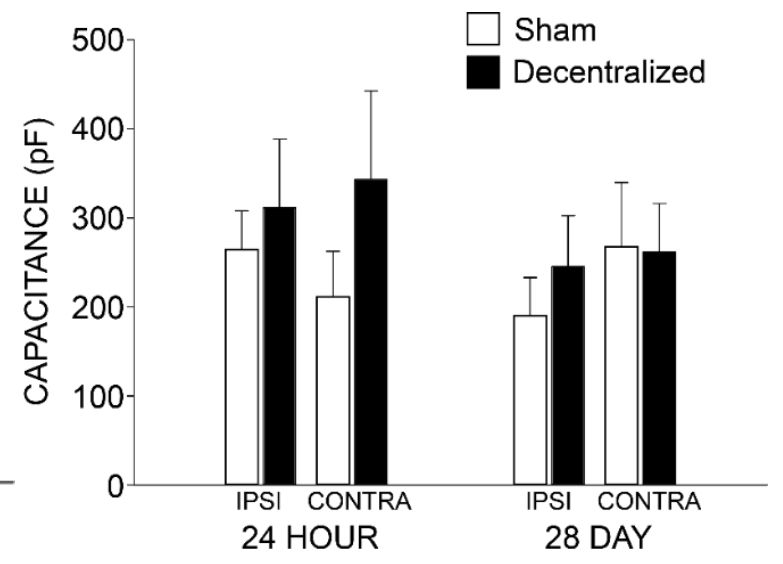

(B)

Figure 3.2 Current densities from decentralized MPG neurons were higher than the corresponding sham groups, however, capacitances were not significantly different between groups.

A. Average current densities of acetylcholine-activated currents in sham vs. decentralized groups (mean + S.E). Data were analyzed using Mann-Whitney Rank Sum tests. * indicates $\mathrm{P}<0.05$. B. Average capacitances of MPG neurons from sham vs. decentralized groups $($ mean + S.E) .

Current densities are current amplitudes of the cells normalized to their capacitances.

Decentralized groups had higher than those of sham groups, however, significant

differences were found only in 24-hour contralateral group $(\mathrm{P}=0.041)$ between sham and decentralized MPGs (Fig 3.2A). Capacitances were not significantly different between sham and decentralized groups in all pairs of comparisons (Fig 3.2B).

Amplitudes of voltage deflections in response to acetylcholine $(1 \mathrm{mM})$ pressure ejections of $0.5 \mathrm{~s}$ in duration were measured in current clamp mode. For those cells that responded with action potentials, the amplitudes of the voltage deflections were measured from the resting potential to the peak of the deflection after the action potential has occurred.

Voltage responses to acetylcholine were generally higher in decentralized groups compared to their sham counterparts, however, statistical significance was found only between sham and decentralized 28-day ipsilateral groups $(\mathrm{P}=0.042)($ Fig 3.3). 
(A)

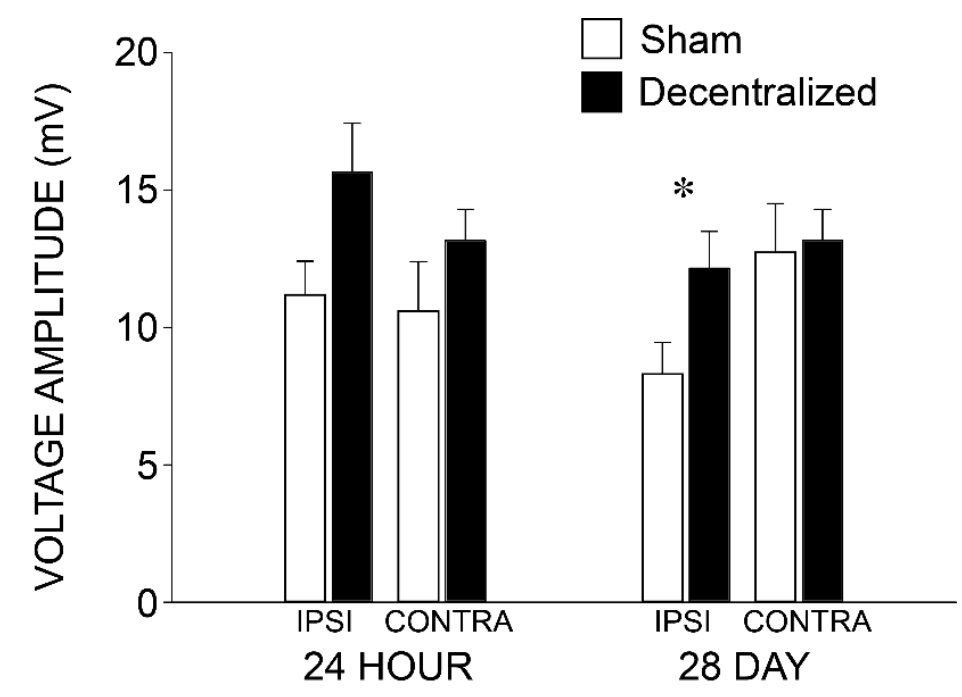

(B)

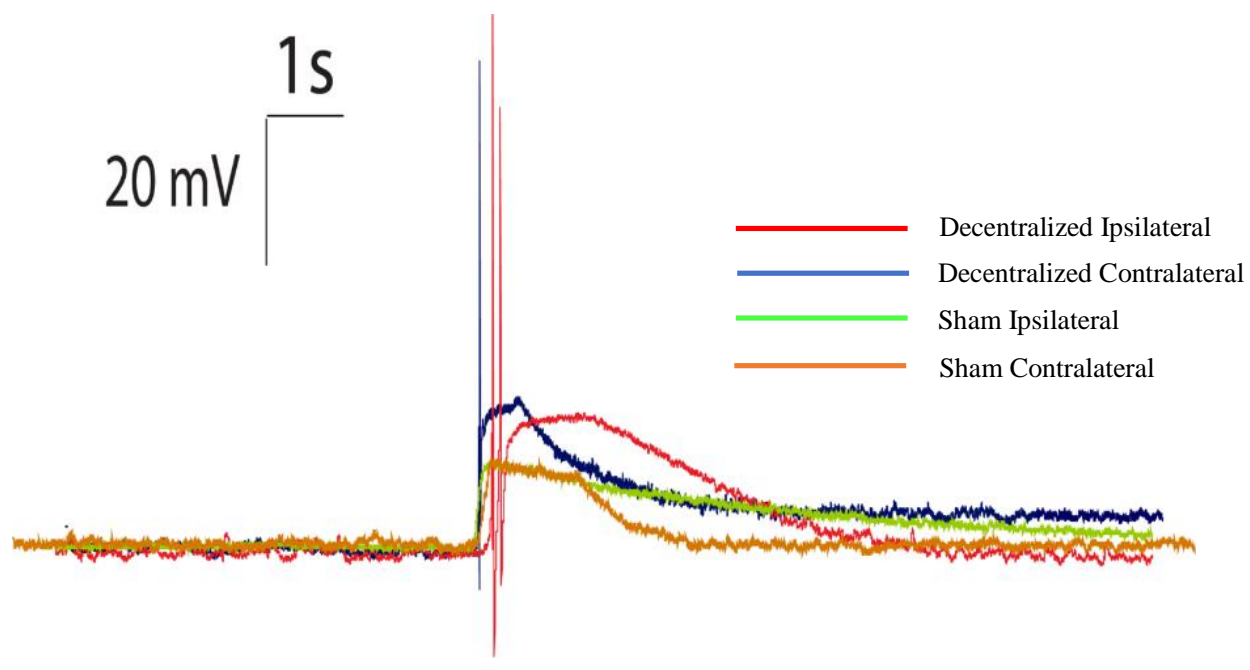

Figure 3.3 MPG neurons from decentralized groups show higher amplitudes of voltage responses than the sham groups, however, statistical significance is observed only between ipsilateral sides of 28-day sham and decentralized groups.

A. Amplitudes of voltage deflections in response to acetylcholine pressure ejections (mean + S.E). * indicates $\mathrm{P}<0.05$. B. Representative traces of voltage deflections from 24-hour groups in response to acetylcholine pressure ejections. 
(A)

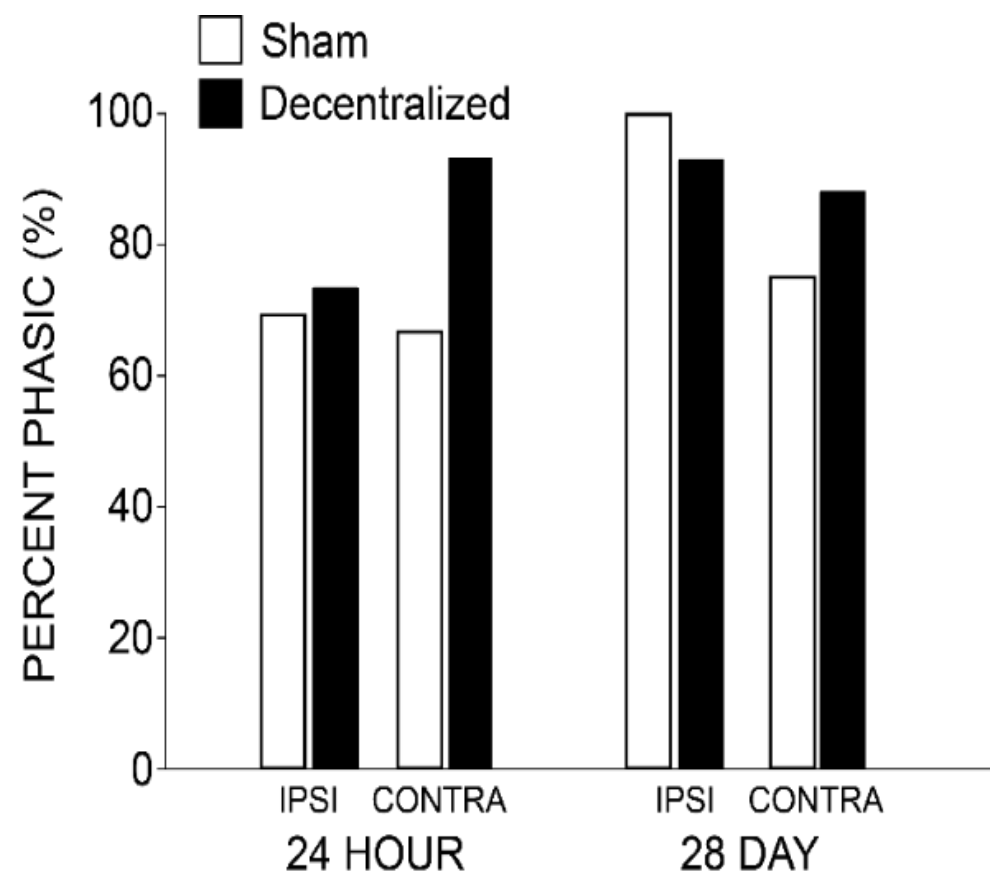

(B)

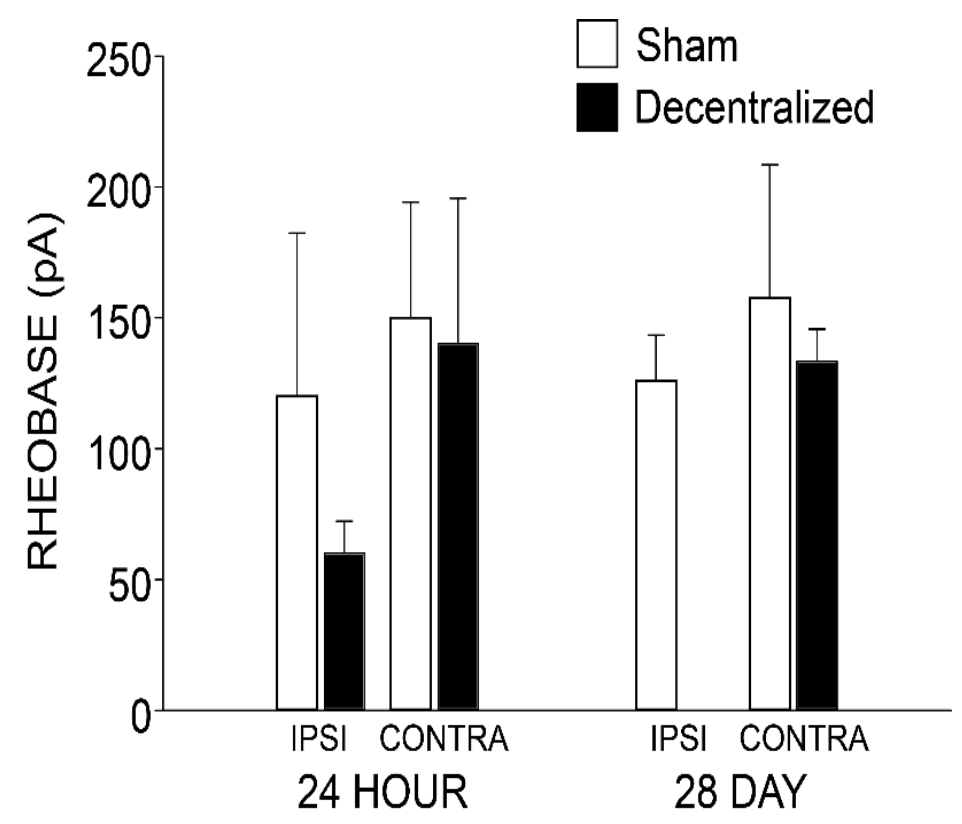

Figure 3.4 Excitability of the MPG neurons do not seem to alter either in terms of number of tonic cells or rheobase currents.

High percentage of the cells in MPG from all groups are phasic. Percentages of phasic and tonic cells do not seem to be different between sham and decentralized groups (Chi-square frequency tests). A.

Average percentages of phasic and tonic cells from sham and decentralized groups. B. Rheobase currents from sham and decentralized groups (except for 28-day ipsilateral decentralized group) (mean + S.E) using current steps. Data are analyzed using unpaired t-tests. 
The firing patterns of the MPG neurons were analyzed across all experimental groups. Intracellular recordings were acquired in current clamp mode from 116 MPG neurons across all experimental groups. The neurons are considered phasic if they respond to depolarizing current injections of $\sim 400 \mathrm{~ms}$ with adapting (one or two) action potentials. Tonic neurons are those that fired action potentials throughout the duration of current injections. High percentage of neurons from all the groups were found to be phasic in the range of $66.67 \%$ (24 hr sham contralateral) to $100 \%$, (28 days sham ipsilateral) while tonic neurons represent a smaller percentage $0 \%$ (28 days sham ipsilateral) to $33.33 \%$ (24 $\mathrm{hr}$ sham contralateral). Despite the data range up to $33.33 \%$ in tonic neurons across groups, Chi-square tests on phasic and tonic cell counts showed no significant differences in the frequency of either type among groups (Fig 3.4A).

We also tested the excitability of MPG neurons by measuring the magnitude of current required for a neuron to fire the first action potential, called rheobase current. No significant differences were found in rheobase currents between sham and decentralized groups for $24 \mathrm{hr}$ ipsilateral (sham $n=3$, decentralized $n=4) 24 \mathrm{hr}$ contralateral (sham $n=4$, decentralized $n=3$ ), and 28 day contralateral (sham $n=4$, decentralized $n=9$ ) groups using unpaired t-tests (Fig 3.4B). We did not have rheobase data for 28 day decentralized ipsilateral group, and hence no comparisons could be made between 28 day sham and decentralized ipsilateral groups. Data for rheobase should also be interpreted with caution due to small sample sizes. 

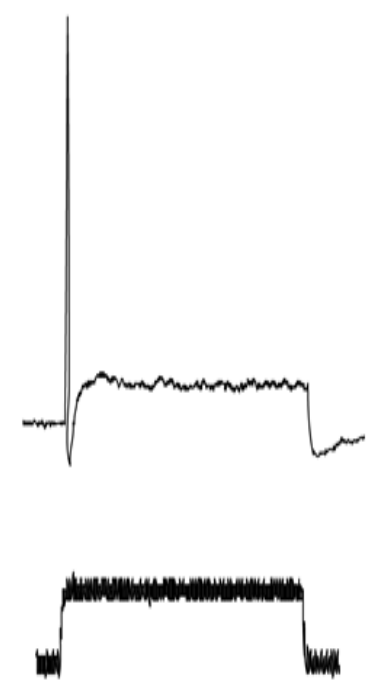

(A)
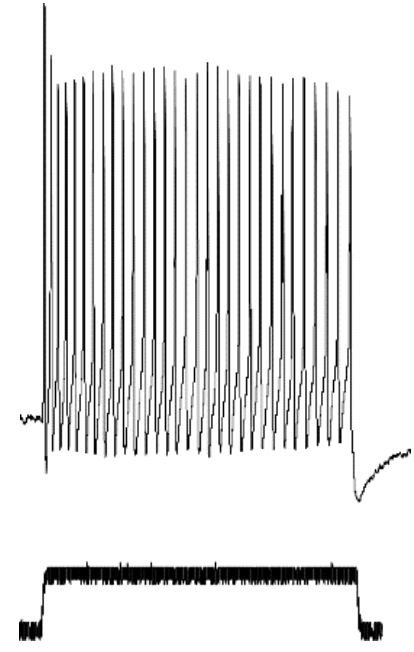

(B)

Trace 3.1 Traces showing example firing patterns in response to current injection. A. shows response from a phasic cell with a single action potential (top trace) to intracellular current injection (bottom trace). B. shows response from a tonic cell with action potentials firing (top trace) throughout the duration of current injection (bottom trace).

(A)

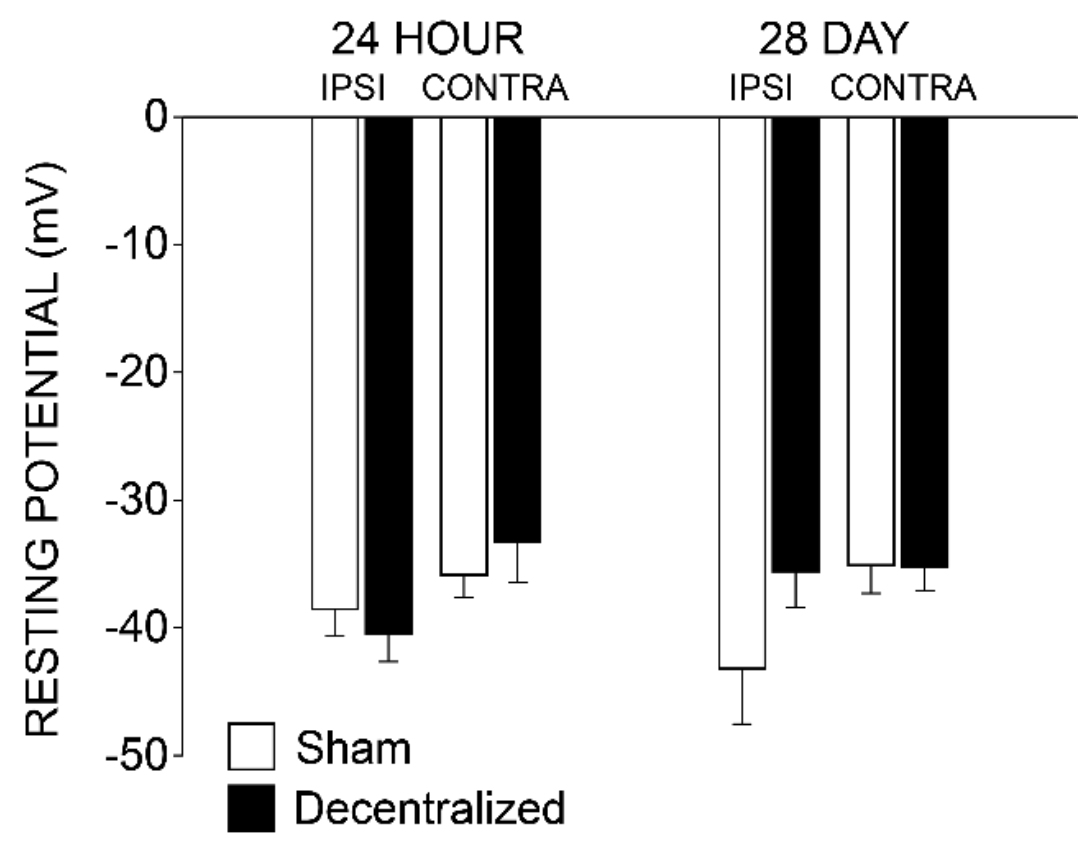


(B)

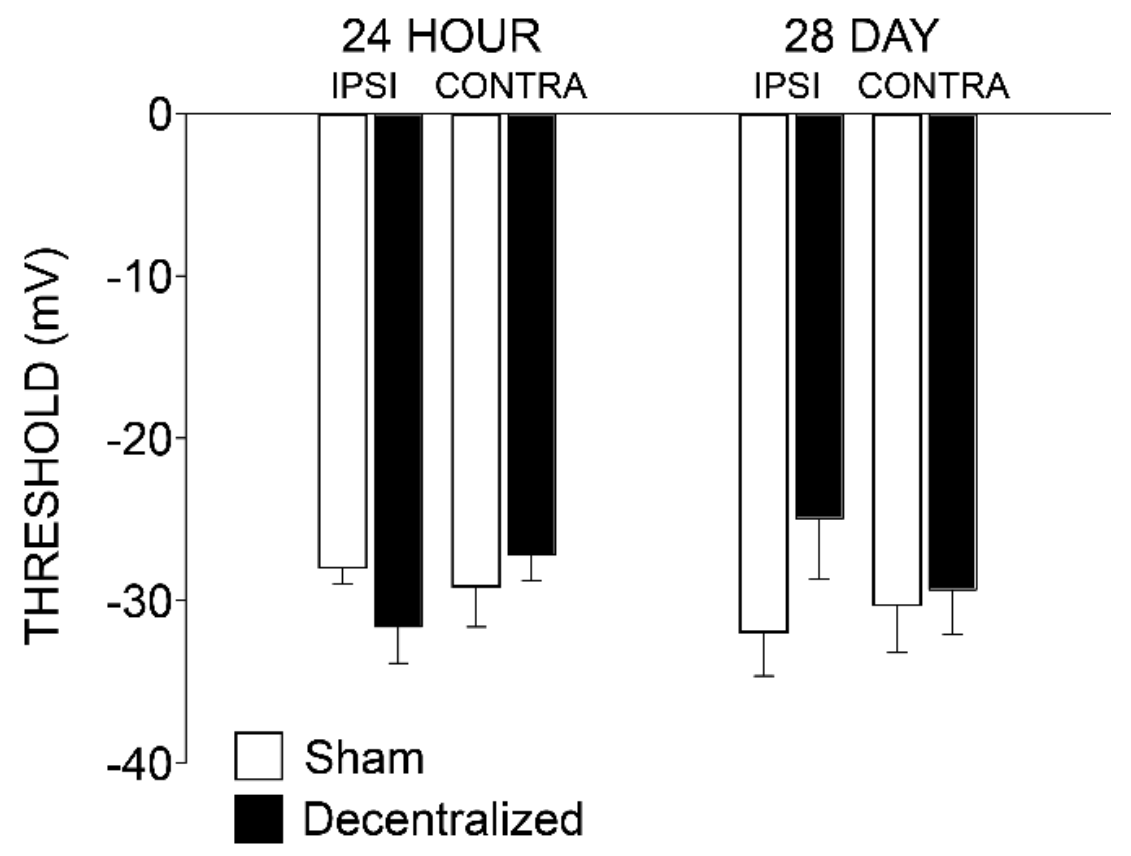

(C)

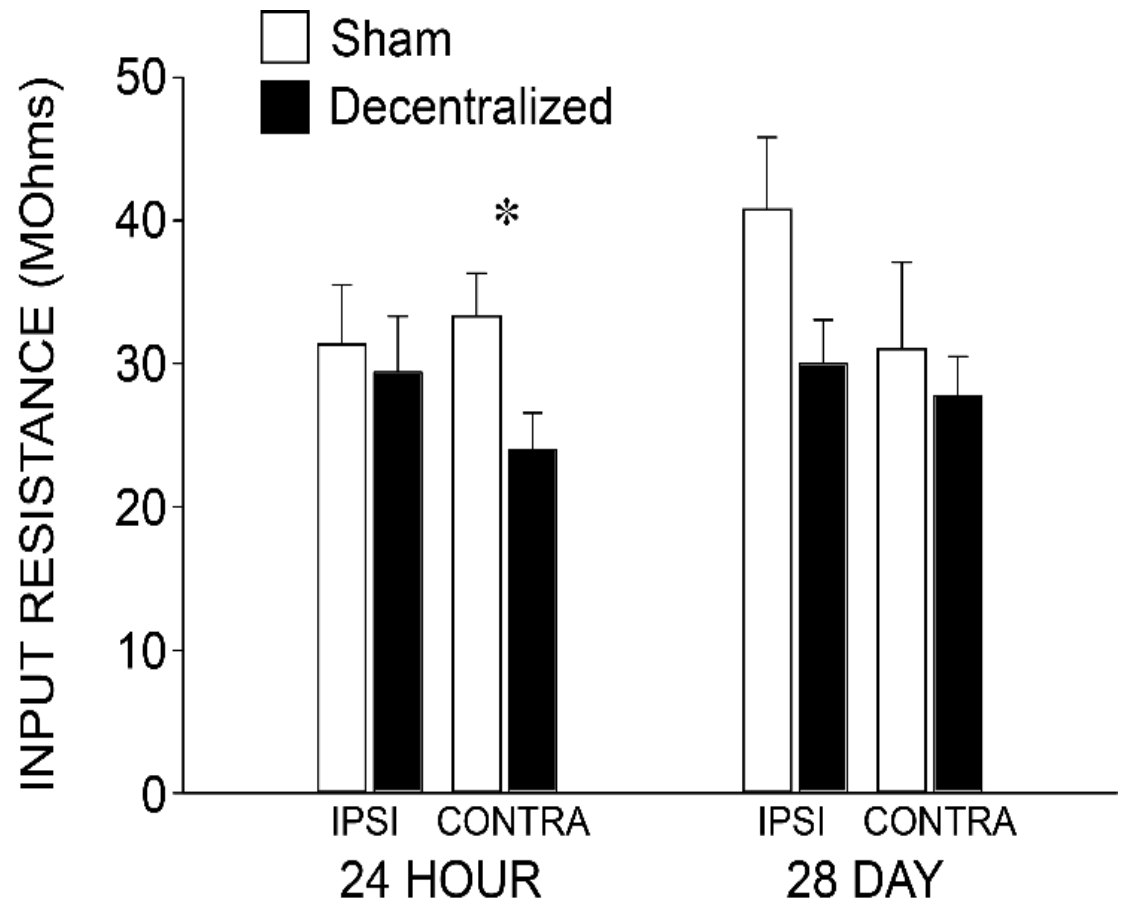


(D)

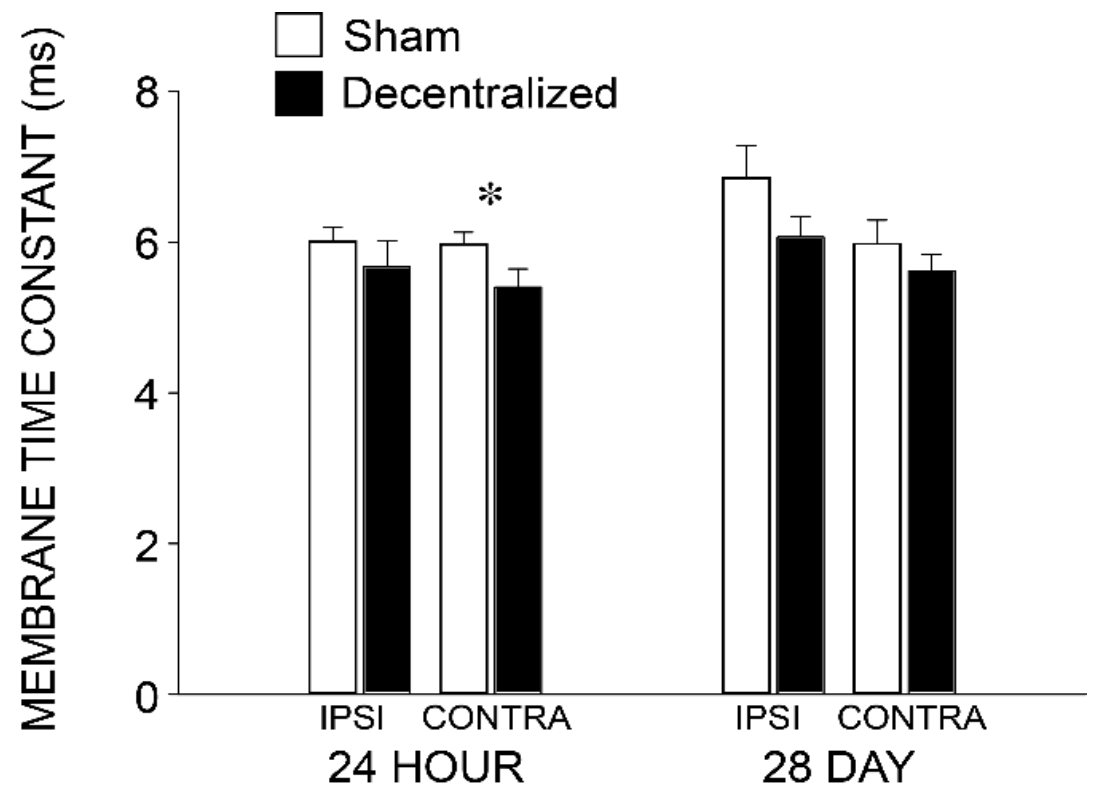

Figure 3.5 Passive membrane properties of MPG neurons from both sham and decentralized groups on both sides show very little differences.

A. Resting membrane potentials from sham and decentralized groups (mean \pm S.E). B. Threshold potentials measured at $10 \mathrm{mV} / \mathrm{ms}$ point on rising slopes of hyperpolarization-induced action potentials (mean \pm S.E). C. Time constants measured by fitting single exponentials of the hyperpolarized voltage traces in current clamp (mean \pm S.E). D. Input resistances calculated from voltage changes in response to hyperpolarizing currents (mean \pm S.E). Data are analyzed using unpaired t-tests and Mann-Whitney Rank Sum tests. * indicated $\mathrm{P}<0.05$.

\begin{tabular}{|l|l|l|l|l|l|l|}
\hline Groups & \multicolumn{3}{|c|}{24 hr Ipsilateral } & \multicolumn{2}{c|}{24 hr Contralateral } \\
\hline & Sham (n) & $\begin{array}{l}\text { Decentralized } \\
(\mathrm{n})\end{array}$ & P value & Sham (n) & $\begin{array}{l}\text { Decentralized } \\
(\mathrm{n})\end{array}$ & P value \\
\hline RMP & $-37(18)$ & $-40(20)$ & 0.454 & $-35(15)$ & $-33(28)$ & 0.565 \\
\hline Threshold & $\begin{array}{l}-27.32 \\
(10)\end{array}$ & $-33.22(16)$ & 0.236 & $-29.14(7)$ & $-27.22(13)$ & 0.501 \\
\hline $\begin{array}{l}\text { Apparent } \\
\text { input } \\
\text { resistance }\end{array}$ & $27.01(18)$ & $28.3(25)$ & 0.712 & $33.32(15)$ & $24.02(28)$ & $0.029^{*}$ \\
\hline $\begin{array}{l}\text { Time constant } \\
\text { 6.13(18) }\end{array}$ & $5.68(25)$ & 0.214 & $6.02(15)$ & $5.57(28)$ & $0.045^{*}$ \\
\hline Rheobase & $90(3)$ & $60(4)$ & 0.629 & $150(4)$ & $140(3)$ & 0.892 \\
\hline
\end{tabular}




\begin{tabular}{|l|l|l|l|l|l|l|}
\hline Groups & \multicolumn{3}{|c|}{28 d Ipsilateral } & \multicolumn{3}{c|}{28 d Contralateral } \\
\hline & Sham (n) & $\begin{array}{l}\text { Decentralized } \\
(\mathrm{n})\end{array}$ & P value & Sham (n) & $\begin{array}{l}\text { Decentralized } \\
(\mathrm{n})\end{array}$ & P value \\
\hline RMP & $-40(5)$ & $-34.7(15)$ & 0.187 & $-36.5^{*}(6)$ & $-35.5(24)$ & 0.971 \\
\hline Threshold & $-31.95(8)$ & $-24.94(6)$ & 0.143 & $-30.29(9)$ & $-29.35(13)$ & 0.822 \\
\hline $\begin{array}{l}\text { Apparent input } \\
\text { resistance }\end{array}$ & $40.77(14)$ & $29.99(15)$ & 0.075 & $\begin{array}{l}24.42 \\
(13)\end{array}$ & $25.32(24)$ & 1 \\
\hline Time constant & $6.85(13)$ & $6.07(15)$ & 0.127 & $5.79(13)$ & $5.39(24)$ & 0.301 \\
\hline Rheobase & - & - & - & $157.5(4)$ & $133.3(9)$ & 0.53 \\
\hline
\end{tabular}

Table 3.2 and Table 3.3 show electrical properties of MPG neurons from sham groups compared to corresponding decentralized groups and $P$ values indicating statistically significance of each comparison.

Electrical properties of MPG neurons were measured in current clamp mode. Median values for the measured electrical properties are described in Tables 2 and 3. The resting membrane potentials (RMP) between sham and decentralized groups were compared using unpaired t-tests or Mann-Whitney Rank Sum tests as appropriate. RMPs were not significantly different between sham and decentralized groups (Fig 3.5A).

Input resistances were measured by dividing the change in voltage by corresponding amplitude of hyperpolarizing current injections. Significant decrease in input resistances in contra lateral ganglia of decentralized animals were found only in $24 \mathrm{hr}$ group $(\mathrm{P}=0.021)($ Fig 3.5C).

Threshold to fire action potentials were measured at the voltage at which $10 \mathrm{mV} / \mathrm{ms}$ point on the rising slope of rebound action potentials were observed. No significant differences in threshold potentials were found between sham and decentralized groups (Fig 3.5B). 
Time constants were measured by single exponential fittings of the hyperpolarized voltage traces in response to hyperpolarizing current injections. Significant decrease in time constant between sham and decentralized groups were found in contralateral group from $24 \mathrm{hr}$ decentralized animals ( $\mathrm{P}=0.045$ ) (Fig 3.5D).

Quantitative analyses of mRNA subunits for neuronal subunits ( $\alpha 2-\alpha 7, \beta 2$ and $\beta 4)$ of nicotinic acetylcholine receptors (nAChRs) were performed using real-time quantitative PCR (q-PCR) method. Ipsilateral and contralateral MPG of animals from each group of $24 \mathrm{hr}$ sham ( $\mathrm{n}=5), 24 \mathrm{hr}$ decentralized ( $\mathrm{n}=7), 28$ day sham ( $\mathrm{n}=6)$, and 28 day decentralized $(n=7)$ were used for the analyses. Data were analyzed using unpaired t-tests or Mann-Whitney Rank Sum tests as appropriate.

Out of all the subunits, $\alpha 3$ and $\beta 4$ subunits showed significant increases in their copy numbers in the MPG of the decentralized animals compared to those of sham animals while $\alpha 4, \alpha 5$, and $\alpha 6$ subunits showed decrease mRNA levels on both ipsilateral and contralateral sides following decentralization (Fig 3.6). 

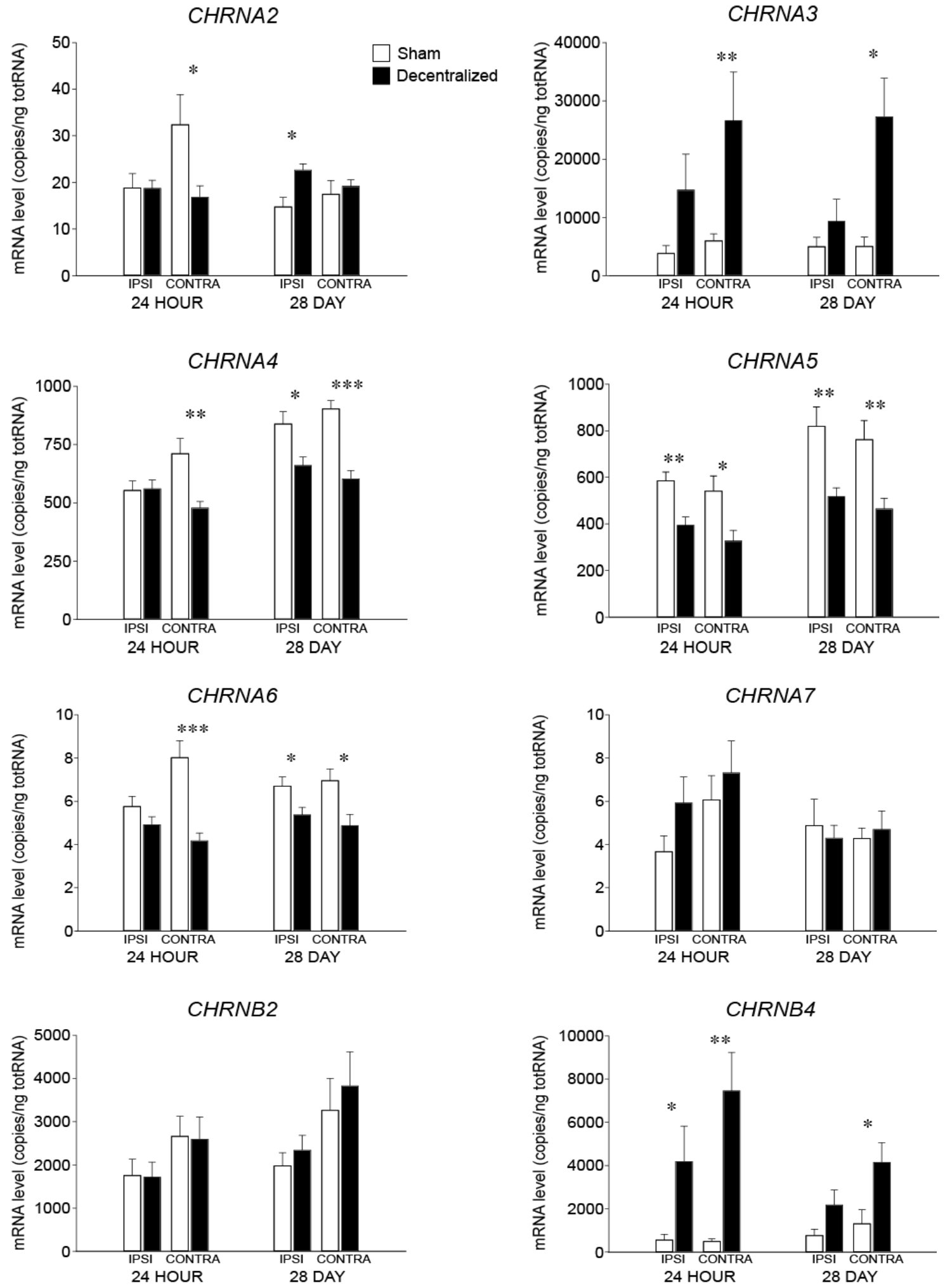

Figure 3.6 qPCR analyses showed changes in neuronal nicotinic acetylcholine receptor subunits.

A. mRNA copy numbers of nAChR subunits in sham vs. decentralized ipsilateral and contralateral MPG at 24 hour and 28 days. B. Correlated expression of Chrna3 and Chrnb4 mRNA subunits across all experimental groups. Data are analyzed using unpaired t-tests or Mann-Whitney Rank Sum tests as appropriate. * indicates $\mathrm{P}<0.05, * *$ indicates $\mathrm{P}<0.005$, and $* * *$ indicates $\mathrm{P}<0.001$. 


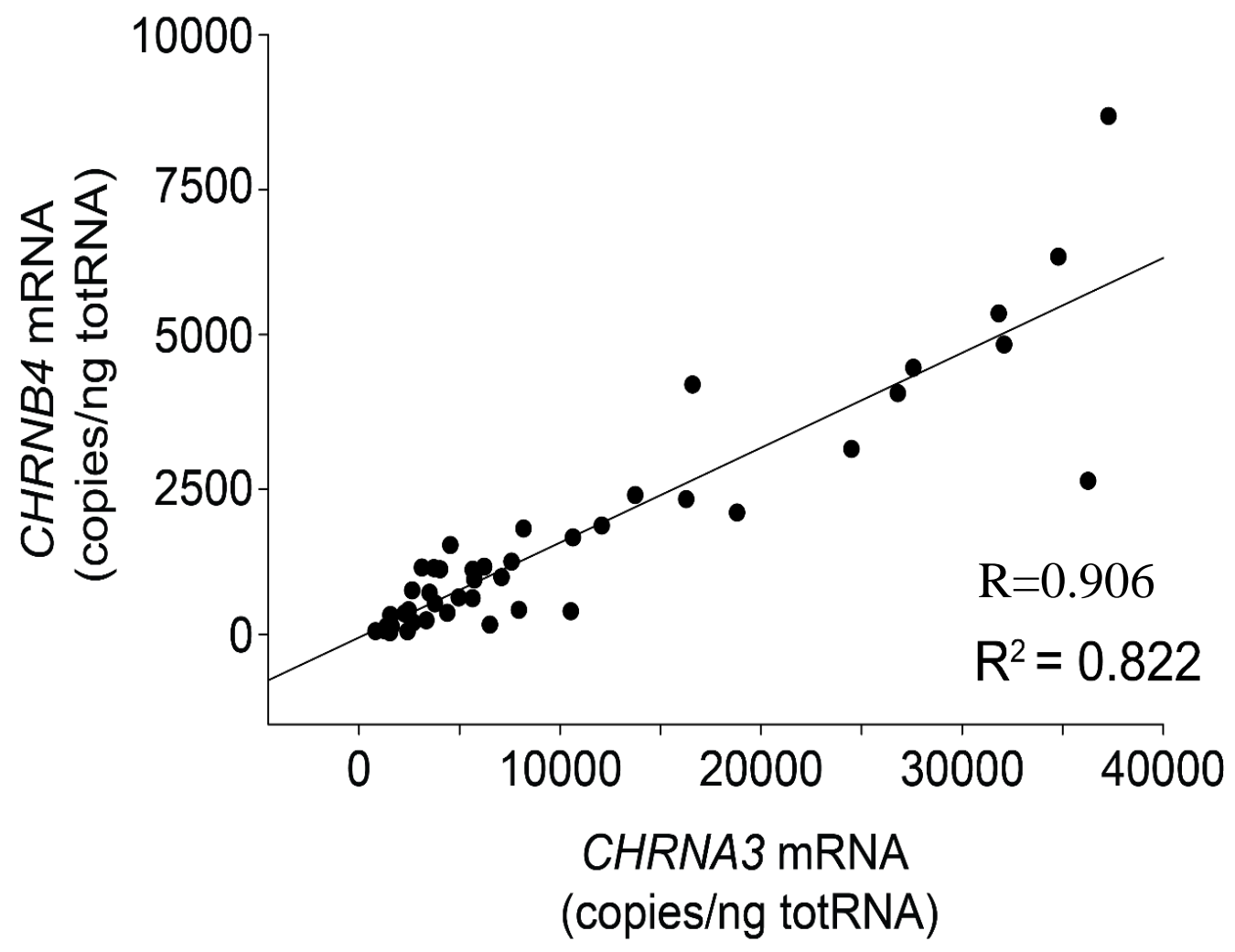

Figure 3.7 mRNA expression levels for CHRNA3 and CHRNB4 genes were found to be positively correlated across all experimental groups.

Data were analyzed using Pearson correlation test.

mRNA copy numbers of Chrnb4( $\beta 4 \mathrm{nAChR}$ subunit) are significantly upregulated in both ipsilateral ( $\mathrm{P}=0.018$ for $24-\mathrm{hr})$ and contralateral ( $\mathrm{P}=0.006$ for $24-\mathrm{hr}, \mathrm{P}=0.05$ for 28 day) MPGs of decentralized animals at 24-hr and on contralateral side at 28-day (Fig 3.6). mRNA levels of Chrna3 ( $\alpha 3 \mathrm{nAChR}$ subunit) were also found to be upregulated on contralateral side of the decentralized ganglia at both 24-hr $(\mathrm{P}=0.028)$ and 28-day $(\mathrm{P}=0.042)$ (Fig 3.6). Out of all the subunits, mRNA expressions of $\alpha 3$ and $\beta 4$ subunits showed positive correlation ( $\mathrm{R}=0.906)$ across all the experimental groups and conditions (Fig 3.7). 
Chrna5 ( $\alpha 5)$ mRNA levels were found to be down-regulated after decentralization at both time points on both ipsilateral ( $\mathrm{P}=0.005$ for both 24 -hr and 28 -day) and contralateral sides $(\mathrm{P}=0.019$ for 24-hr and $\mathrm{P}=0.009$ for 28-day (Fig 3.6). Chrna4 ( $\alpha 4)$ and Chrna6 ( $\alpha 6)$ subunits were also significantly down-regulated after decentralization in all groups except for the ipsilateral groups at 24-hr (Fig 3.6). $\mathrm{P}$ values for Chrna4 groups are $\mathrm{P}=0.005$ for 24-hr contra, $\mathrm{P}=0.021$ for 28 -day ipsi, and $\mathrm{P}=<0.001$ for 28 -day contra. $\mathrm{P}$ values for Chrna6 groups are $\mathrm{P}=<0.001$ for $24-\mathrm{hr}$ contra, $\mathrm{P}=0.033$ for 28 -day ipsi, and $\mathrm{P}=0.023$ for 28-day contra. Chrnb2 ( $\beta 2)$ subunit levels did not change significantly after decentralization (Fig 3.6). mRNA levels of Chrna7( $\alpha 7$ ) subunits were negligible in all groups (Fig 3.6).

\subsection{Discussion}

We studied the acute and chronic effects of unilateral decentralization on synaptic and electrical properties of mouse major pelvic ganglion (MPG) neurons. Loss of input to mouse major pelvic ganglia (MPG) causes increased in synaptic strength of cholinergic MPG neurons in both acute stage (24-hours) and chronic stage (28-days) post-injury while the excitability and the electrical properties of the neurons were preserved. Both decentralized (ipsilateral) MPG and the intact contralateral MPG which is located on the other side of the bladder in mice showed increased upregulation of nicotinic acetylcholine receptors (nAChRs) in decentralized mice.

In response to acute loss of inputs, we expected decreased magnitude of currents in response to acetylcholine pressure ejections, however, our results indicated increased magnitude of currents in decentralized MPG neurons compared to the sham controls. One 
possible explanation for this could be due to increased sensitivity of the post-synaptic membrane in response to loss of presynaptic inputs called 'denervation supersensitivity'. The phenomena of denervation supersensitivity have been first been reported in denervated muscles by Cannon \& Rosenbleuth in 1949 (Thesleff \& Sellin, 1980). The supersensitivity of the muscle after denervation has been shown to be accompanied by increased in the number of nAChRs and change in the distribution of nAChRs (Cannon \& Rosenbleuth, 1949; Thesleff \& Sellin, 1980). Other proposed mechanisms of denervation-induced super-sensitivity in skeletal muscle as well as in autonomic ganglia include reduction of acetylcholinesterase enzyme in extrajunctional receptors (Fambrough, 1979; Axelsson and Thesleff 1959; Streichert \& Sargent, 1992), activation of genetically-controlled protein synthesis (Fambrough ,1979), changes in permeability or ionic conductance of the post-synaptic membrane (Fleming et al., 1973; Thesleff \& Sellin, 1980), and post-synaptic intracellular calcium concentration (Thesleff \& Sellin,1980).

Studies in autonomic ganglia have also shown increase in sensitivity to neurotransmitters as well as increase in transcriptional regulators after loss of preganglionic inputs. A bilateral deafferentation study of mouse MPGs by Nangle \& Keast (2009) reported upregulation of c-Jun, an early response transcription factor, in MPG neurons of decentralized ganglia. A denervation study in frog cardiac ganglion also showed increased sensitivity to acetylcholine, however, this was no longer the case when acetylcholinesterase inhibitor was present (Streichert \& Sargent, 1992). Another denervation study in cat superior cervical ganglia showed increase in sensitivity of the ganglionic neurons to acetylcholine which was not observed with cholinesterase-resistant 
stimulants, implying reduction in acetylcholinesterase to be the cause of increased sensitivity to acetylcholine (Brown, D.A, 1969).

In our study, we found that the increase in synaptic strength in decentralized ganglia were also accompanied by significant increase in mRNA transcript expression for $\alpha 3$ and $\beta 4$ subunits while other $\alpha$ subunits and $\beta 2$ subunit showed either decrease or no significant change after decentralization. The identity of nAChR subunits and their composition in specific subtypes determine different agonist affinities, and their permeability to cations (Parker et al.,1998). When ACh binds to nAChRs, the receptors undergo conformational change from closed to open state which allows the influx of cations ( $\mathrm{Na}+$ and $\mathrm{Ca} 2+)$ as well as efflux of $\mathrm{K}+$ in nonspecific manner (Wonnacott \& Barik, 2007). Each neuronal nAChR subunit has varying degrees of permeability to Ca2+ (Sargent, 1993; DajasBailador \& Wonnacott 2004). The influx of $\mathrm{Ca} 2+$ can depolarize the membrane voltage which in turn could activate the voltage-gated Ca2+ channels (Parker et al., 1998). This $\mathrm{Ca} 2+$ can also calcium-activated $\mathrm{K}+$ channels as well as activate second-messenger mediated signaling pathways (Sargent, 1993). $\alpha 3$ subunits, a predominant ligand-binding subunit in autonomic ganglia, is shown to mediate cation conductances in response to ACh while the $\beta$ subunits are known to play a major role in deciding agonist affinity (Skok, 2002; Wang et aal.,2002). Physiological experiments involving subunit specific nicotinic acetylcholine receptor blockers suggest that $\alpha 3$ and $\beta 4$ subunits seem to be the major subunits involved in nicotinic transmission at the mouse MPG (Skok, 2002; De Biasi et al.,2000; Kyi, unpublished). Gene knockout studies have also indicated that $\alpha 3$ and $\beta 4$ subunits are important in normal functioning of autonomic ganglia (Wang et al., 2002). 
The correlated mRNA expressions of $\alpha 3$ and $\beta 4$ subunits that we found in this study is another evidence pointing to the possibility that these two subunits are co-regulated in this ganglion and hence may form a functional pair. We do not have direct evidence to show the presence of receptor proteins on the cell membrane, however, our mRNA studies suggest that the increase in sensitivity to acetylcholine could be due to increased availability of functional $\alpha 3$ and $\beta 4$ subunits on the post-synaptic membrane. Denervation studies in muscle reported that increase in sensitivity following denervation can be blocked either by inhibitors of protein synthesis or by blocking RNA synthesis which suggests that the increased sensitivity stems from newly synthesized receptors in the muscle membrane (Fambrough, 1979).

Denervation-induced hypersensitivity to neurotransmitter receptors have also been observed in spinal motoneurons and interneurons after spinal cord injury (Murray et al. 2010; Li et al. 2004; Husch et al., 2012; Barbeau and Bedard, 1981). Studies have shown that hypersensitivity of motoneurons below the injury to 5-HT (Serotonin) contributes to the spasticity associated with SCI (Murray et al., 2010; Li et al., 2004; Barbeau and Bedard, 1981). Loss of supraspinal projections after SCI reduce serotonin concentration at the motoneuron synapses below injury. Subsequently, upregulations of 5-HT2C receptors and reduction in SERT (Serotonin transporters) were observed with development of hypersensitivity to 5-HT (Murray et al., 2010).

In the present study, the increased amplitudes of current responses to acetylcholine were more pronounced in contralateral ganglia compared to the decentralized ganglia at both acute and chronic stages at both time points. This finding poses a question of whether 
there is a co-regulatory mechanism for both sides in response to unilateral decentralization which causes higher increases in current amplitudes on the contralateral side. Another possibility is that there is a compensation from the contralateral ganglia to compensate for the loss of activity from the decentralized ganglia. If this was the case, there could be certain feedback communication between the two ganglia or the information could originate from the bladder and conveyed to the contralateral intact ganglia.

Neurogenensis in the contralateral ganglia, increased NGF level, and increased synaptic activity have been reported in ganglionic decentralization and ganglionectomy studies. Hypertrophy of contralateral rat MPG neurons following unilateral ganglionectomy (removal of a whole MPG and hence severing the connections between the ganglion and the target organs) was reported by Gabella and Uvelius (1993). Another unilateral ganglionectomy experiments in rats by Gabella et al., (1992) reported the doubling in the amount of bladder muscle innervated by the contralateral intact ganglion with hypertrophied neurons. Another ganglionectomy study in male rats also showed increased density of synaptic connections in addition to hypertrophy of contralateral MPG neurons (Aldahmash, A. \& Atteya, M, 2011). There has also been reports of sprouting of ganglionic neurons after decentralization observed by immunostaining studies (Kepper\&Keast, 1998), and the proposed stimuli for sprouting is increased secretion of neurotrophic factors by bladder in response to loss of preganglionic input (Kepper\&Keast, 1998). 
One could wonder why the decentralized MPG neurons survived over long periods. Studies have reported contribution afferent inputs from target organs in cell survival. During development, loss of target organ resulted in pronounced neuronal degeneration in ciliary ganglion of the chick embryo, and the authors alluded to possible influence of target-derived neurotrophic factors in regulating neuronal survival in addition to afferents (Furber et al.,1987). Neuregulin 1 has also been reported to play a role in upregulating the nAChR expression in MPG neurons of adult rats through the ErbB2/ErbB3-PI3K-MAPK signaling cascade (Kim et al., 2013). Although we do not have direct evidence in this study, there is a possibility that the decentralized MPG neurons were still receiving inputs from some of the bladder afferents or the postganglionic axons of the contralateral ganglia. The possibility of postganglionic axons from the intact ganglion growing across the bladder and extending into postganglionic nerves of the decentralized ganglia was also suggested by unilateral decentralization of bladders in cats (De Groat \& Kawatini, 1989).

Expression of nAChRs and their associated ACh-induced currents in MPG neurons have been shown to be affected not only by decentralization but also by axotomy as well. Girard et al., have shown downregulation of nAChRs a3 and B4 in the male mouse MPG after transection of postganglionic cavernous nerve which recovered 30 days after injury (Girard et al., 2013). We also found in our decentralization study that at 28 days postinjury, the synaptic strength seems to be decreasing towards the sham-control levels and the significant difference disappears for the contralateral ganglia. According to these studies, decentralization and axotomy regulate $\mathrm{nAChR}$ expressions in opposite directions at least during the acute injury period. 
What could be the functional implications of loss of central input to the MPG neurons? A study by Persyn et.al., 2016 examined the role of MPG in bladder function in a series of decentralization and MPG removal experiments in rats. The study did not find significant changes in bladder pressure and non-voiding activities following bilateral transections of pelvic and hypogastric nerves, however, removal of MPG reportedly increased the baseline pressure, decreased compliance and increased amplitude of non-voiding contractions (Persyn et al. 2016). The authors suggested that this non-voiding activity is modulated at the MPG, a possible peripheral modulatory center, with a possible circuitry from the target organ with sensory feedback (Persyn et.al., 2016). Similar effects on nonvoiding activity and increased baseline pressure was observed when Hexamethonium was applied to decentralized bladder in a study by Sugaya and de Groat in rats suggesting the existence of hexamethonium-sensitive peripheral mechanism which regulate bladder activity (Sugaya \& de Groat, 2007). These studies indicate an important role the MPG neurons play in regulation of bladder function and hence imply that alteration of inputs or loss of inputs to these neurons can have functional implications.

We also examined excitability and passive properties of the MPG neurons in both decentralized and contralateral ganglia. Changes in nAchR numbers can alter the membrane properties due to changes in permeability of ions across the membrane through the receptors (Parker et al., 1998). However, we did not find significant changes in passive properties of the MPG neurons in either decentralized or contralateral ganglia at either time points. This was also the case with V2a spinal interneurons after SCI, when there was hypersensitivity to 5-HT but no changes in intrinsic excitability or action potential properties were observed (Husch et al., 2012). Preservation of membrane 
properties while there was up-scaling of AMPA receptors has also been reported after chronic, natural inactivity in respiratory motoneurons in adult American bullfrogs (Santin et al., 2017).

Our study showed that loss of input to mouse MPG results in increased synaptic transmission of the MPG neurons, together with compensation from the contralateral MPG, and this increase is also reflected at the level of mRNA transcript numbers for the major subunits involved in the synaptic transmission. This information is important for understanding the role of major pelvic ganglia in neural control lower urogenital tract. We can also learn how the MPG neurons respond to immediate loss of input which is a valuable information in case of injuries of preganglionic pelvic and hypogastric nerves as well as more distal injury that can alter the preganglionic input to the ganglia such as spinal cord injury. Future studies could focus on the mechanisms of selective upregulation of $\alpha 3$ and $\beta 4$ receptors in MPG after decentralization, the possible connections from the contralateral ganglia as well as explore feedback circuitry from the bladder to the MPG. 


\section{CHAPTER 4 \\ EFFECTS OF SPINAL CORD INJURY ON SYNAPTIC, PASSIVE, AND ACTIVE PROPERTIES OF NEURONS OF MOUSE MAJOR PELVIC GANGLIA}

\subsection{Introduction}

Lower urinary tract function is one of the autonomic functions that is impaired after spinal cord injury (SCI) (de Groat \& Yoshimura, 2012; Ditunno Jr. \& Formal, 1994). Acute SCI results in areflexic bladder and complete urinary retention while patients with chronic SCI suffer from hyperreflexic bladder, incontinence and inefficient voiding (de Groat, 1995; Yoshimura \& de Groat, 1997). Colon function has been reported to be less affected by spinal cord injury (Yoshimura \& de Groat 1996). While extensive SCI research has been done on locomotor recovery, relatively limited amount of research has been done on the autonomic dysfunctions associated with SCI. Autonomic dysfunctions impose significant impact on day to day functions of patients and most people with SCI were reported to desire recovery of autonomic functions over regaining of locomotor functions (NINDS, 2013).

Spinal cord injury disrupts voluntary control and the normal reflex pathways that coordinate bladder and sphincter function (de Groat, 1995; de Groat \& Yoshimura, 2012). After spinal cord injury, the absence of supraspinal inputs from brainstem micturition centers cuts the voluntary control of bladder function (de Groat 1995; Yoshimura \& De Groat 1996). Initially, SCI results in areflexic bladder due to absence of reflex activity below the level of lesion. However, the activity of sphincter persists or rapidly recovers, and the presence of sphincter tone causes urinary retention. Reflex detrusor activity reappears after 2-12 weeks and involuntary reflex contractions 
responding to bladder filling causes involuntary voiding (de Groat, 1995; Yoshimura, 1999). Disruption of coordination between bladder and sphincter causes simultaneous contractions of the bladder and striated urethral sphincter, resulting in inefficient voiding, involuntary contractions, striated sphincter dys-synergia (de Groat, 1993; 1995; Yoshimura, 1999).

The major pelvic ganglia (MPG) are heterogenous ganglia containing parasympathetic and sympathetic postganglionic neurons that innervate the urogenital organs and distal colon (de Groat \& Yoshimura, 2010; Jobling \& Lim, 2008), and thus are one of the major sources of bladder innervation. MPG receives cholinergic parasympathetic input from the preganglionic neurons in the sacral cord through pelvic nerve and sympathetic cholinergic inputs from those in the lumbosacral cord through the hypogastric nerve (de Groat, 1995; de Groat \& Yoshimura, 2015). Nicotinic cholinergic transmitter system is the major system involved in ganglionic parasympathetic transmission at the MPG neurons that innervate the bladder (Skok, 2002; De Biasi, 2002; Park et al., 2006; Xu et al., 1999).

Most research has been done on the changes in sensory afferent pathways of micturition reflex as well as changes in morphology of sensory neurons in dorsal root ganglia (DRG) after spinal cord injury (Yoshimura \& Groat, 1997; de Groat \&Yoshimura, 2010; de Groat et al, 1990; Takahashi et al.,2013). However, electrophysiological properties of MPG neurons after spinal cord injury have not been studied. It is important to understand the physiology of efferent ganglionic transmission in normal and injured states not only to complete our understanding of the system but also to discover new therapeutic measures. Understanding the physiology and expression patterns of specific subunit or 
subtypes of nAChR before and after injury will be valuable in identifying potential therapeutic agents with more efficiency and fewer generalized effects in treating bladder disorders associated with spinal cord injury. The use of mouse as a model system is also an advantage since there is an availability of transgenic mice models for specifically studying parasympathetic neurons.

The goal of this project is to understand the effects of spinal cord injury on synaptic, intrinsic and action potential properties of parasympathetic efferent neurons at the MPG. Although MPG is a heterogenous ganglion containing neurons that innervate the bladder, genitals, and the colon, this study will relate mostly to bladder function since colon functions were reported to be less affected by spinal cord injury, and majority of the MPG neurons innervate the bladder (Yoshimura \& Groat 1997; Keast et al., 1989; Keast \& de Groat, 1989). We will be investigating the effects of spinal cord injury on the synaptic transmission of parasympathetic neurons that control micturition in both acute and chronic stages. We hypothesize that spinal cord injury will alter the properties of MPG neurons as a result of altered presynaptic input from the spinal autonomic neurons after spinal cord injury.

\subsection{Materials and Methods}

\subsection{1 $\quad$ Animal numbers}

Adult male and female transgenic mice (B6.Cg-Tg (RP23-268L19-EGFP) 2Mik/J) from the Jackson Laboratory were used for this study $(\mathrm{N}=44)$. These animals have cholinergic neurons labeled via GFP expression driven by a Choline Acetyltransferase (ChAT) promoter. Animals were grouped into four experimental groups- acute spinal transections (3-day post transection), 3-day laminectomy, chronic spinal transections (28-day post- 
transection), and 28-day laminectomy. The 3-day and 28-day periods were chosen for acute and chronic phases as 3-day is after the 24-hour initial spinal shock of injury, but early enough for recovery of reflex mechanisms, and reflex mechanisms have appeared at 28-day post- injury in rodent models (Bareyre et al., 2004; Smith \& Weaver, 2001; Kruse et al., 1995)

All experimental procedures were approved by the University of Missouri Institutional Animal Care and Use Committee and conformed to the National Institutes of Health Guide for the Care and Use of Laboratory Animals.

\subsubsection{Laminectomy surgical procedures}

A midline incision of $1.5-2 \mathrm{~cm}$ was made on the dorsal midline of the mouse beginning from the middle of the shoulder blades in rostral to caudal direction. The superficial fat underneath the skin was partially removed. The prominent vessel, usually found in the space between the $5^{\text {th }}$ and $6^{\text {th }}$ thoracic dorsal processes was located. The vertebral levels were counted up to T8, and the paravertebral muscles were cut bilaterally from $\mathrm{T} 7$ to T10, staying as close as possible to the spinous processes and parallel to the midline. The muscles between $\mathrm{T} 7$ and $\mathrm{T} 8$, then $\mathrm{T} 8$ and $\mathrm{T} 9$ were cut. Then, the $\mathrm{T} 8$ spinal process was removed, and the ligament between T8 and T9 was cut. An initial small cut was made from the opening created when the ligament was removed. The cut continued in a rostral direction all the way to the gap between T7 and T8. The same cutting procedure was followed on the opposite side. Then, the T8 vertebra was removed. The periosteum was removed after the cord between T10 and T11 spinal segment was exposed, and bone debris were cleared off the cord. 


\subsubsection{Spinal transection surgical procedures}

Complete transections were done at the thoracic 8 (T-8) vertebra level, between T10-T11 levels of the spine. After the laminectomy was completed and periosteum removed, two drops of lidocaine solution were placed on the surface of the dura to prevent reflex movement due to external stimulation to the cord. The dura was gently lifted off the dorsal surface of the spinal cord and a hole ( $\sim \mathrm{mm}$ in width) was made for complete transection of the cord. The spinal cord was cut transversely between T10 and T11 spinal segments extending from the dorsal surface and moving ventrally. Complete transection around the circumference of the spinal cord was confirmed by using fine forceps or blunt probe. Care was taken not to cut through the ventral dura. A small piece of Gelfoam was placed in between the cut ends. The muscle was sutured using 6-0 microfilament suture. The skin was stapled using the auto-clip applicator.

\subsubsection{Post-surgical bladder management}

All spinal cord transected animals lost reflex bladder emptying after the surgery. All spinal transected animals received manual urine expressions by having their bladders gently squeezed twice a day until euthanization.

\subsubsection{Tissue isolation for in-vitro recordings}

MPGs were dissected out from the animals after euthanizing with overdose of isoflurane inhalation. The MPGs were then pinned down in a sylgard dish with oxygenated HEPES buffer solution containing, (in $\mathrm{mM}$ ) $\mathrm{NaCl}, 146 ; \mathrm{KCl}, 4.7 ; \mathrm{MgSO} 4,0.6$; $\mathrm{NaHCO} 3$,1.6; NaH2PO4, 0.13; $\mathrm{CaCl} 2$, 2.5; Glucose, 7.8; HEPES, 20), adjusted to pH 7.3 (Jobling and 
Lim, 2008). The saline was preheated to $33-35^{\circ} \mathrm{C}$ and aerated with oxygen before being continuously perfused into recording chamber for in-vitro intracellular recordings.

\subsubsection{In-vitro electrophysiology recordings}

The dissected MPGs were de-sheathed and cleaned off from connective tissue and fat. The ganglia were pinned down on a piece of sylgard glued in a $1 \mathrm{ml}$ recording chamber. GFP-labelled parasympathetic neurons were identified by visualizing the preparation under GFP filter of $470 \mathrm{~nm}$ wavelength. Intracellular recordings were made by impaling individual labelled neurons with sharp glass electrodes. The recording electrodes contain intracellular solution of $3 \mathrm{M} \mathrm{KCl}$ solution and has resistances in the range between 15 M $\Omega$ - 30 M $\Omega$. AxoClamp 900 A (Molecular Devices) amplifier was used for clamping voltages and recording the signals and Digidata 1440A (Molecular Devices) digitizer was used to digitize the signals at $10 \mathrm{kHz}$. The resting membrane potential and voltage responses to ACh pressure ejection puffs were measured in Bridge mode. Current responses were measured in dSEVC (Discontinuous single electrode voltage clamp) mode by clamping each neuron under study at $-50 \mathrm{mV}$ and at the frequencies between 3$5 \mathrm{kHz}$. The signals were acquired in Clampex 10.6 software and data was analyzed in Clampfit 10.6 program.

\subsubsection{Excitability test}

Depolarizing and hyperpolarizing current step protocols were used in bridge mode to measure the membrane and action potential properties of MPG neurons. Depolarizing and hyperpolarizing current steps up to $700 \mathrm{pA}$ and $-700 \mathrm{pA}$ were injected directly into the cells in increments of $60 \mathrm{pA}$ and $-60 \mathrm{pA}$ steps, $400 \mathrm{~ms}$ in duration. The firing patterns of action potentials and their properties were measured. Depolarizing voltage ramp protocol 
with duration of $500 \mathrm{~ms}$ from 0 to $+1 \mathrm{nA}$ were used to measure the rheobase and threshold voltage for action potential firing.

\subsubsection{Nerve stimulation and synaptic properties test}

To detect any frequency dependent modulation, a suction electrode (A-M Systems, Sequim, WA) was used to electrically stimulate pelvic nerve at various frequencies using A-M Systems Isolated Pulse Stimulator, Model 2100 (Carlsborg, WA). The pelvic nerve was stimulated at frequencies of $1 \mathrm{~Hz}, 2 \mathrm{~Hz}, 5 \mathrm{~Hz}, 10 \mathrm{~Hz}$, and $20 \mathrm{~Hz}$ repetitive stimuli with pulse duration of $0.5 \mathrm{~ms}$. Intracellular recording electrodes were used to measure excitatory postsynaptic potentials (EPSPs) responses from individual neurons to pelvic nerve stimulation at resting membrane potentials, and excitatory postsynaptic current (EPSCs) in response to the nerve stimulation were measured by clamping each neuron at $-50 \mathrm{mV}$ using discontinuous single-electrode voltage clamp (dSEVC). The presence of frequency-dependent synaptic modulations was measured by comparing EPSP and EPSC amplitudes at various frequencies of repetitive stimuli.

To investigate synaptic recruitment from MPG neurons, we stimulated the pelvic nerve with individual pulses of $0.5 \mathrm{~ms}$ at various stimulus strengths $(1 \mathrm{~V}-30 \mathrm{~V})$. The presence of synaptic recruitment was observed by comparing the EPSP amplitudes at each stimulus intensity.

\subsubsection{Acetylcholine pressure ejections}

Picospritzer (General Valve Corporation, Picospritzer II) was used for pressure ejection of acetylcholine. A glass electrode was filled with Acetylcholine $1 \mathrm{mM}$ (Acros Organics) and the pressure was set around 6-10 psi. Duration for each pressure puff is $0.5 \mathrm{~ms}$. The 
pressure ejection electrode was placed about $50 \mu \mathrm{m}-75 \mu \mathrm{m}$ away from each cell under study (Fig 4.1).

\subsubsection{Real-time quantitative polymerase chain reactions (qPCR)}

Following electrophysiology experiments, MPG were collected directly into Trizol buffer (Invitrogen) and stored at -80C until RNA extractions.Total RNA was isolated from MPGs using Trizol according to the protocol provided by the manufacturer (Invitrogen). cDNA was generated from 100 ng total RNA primed with a mixture of oligo-dT and random hexamers that was reverse transcribed using qScript reverse transcriptase (QuantaBio). The final volume of the reverse transcription reaction was $20 \mu 1$, and contained a final concentration of $2.5 \mathrm{ng} / \mu 1$ random hexamers, $2.5 \mu \mathrm{M}$ oligo-dT, $40 \mathrm{U}$ of RNase inhibitor, and $200 \mathrm{U}$ of reverse transcriptase. Following heat inactivation of the enzyme, samples were diluted in ultrapure water to a final volume of $175 \mu \mathrm{l}$ before this template was used in qPCR analyses. From each cDNA pool generated from $100 \mathrm{ng}$ of total RNA, we were quantified at least 15 different gene products.

We designed or modified, and independently validated primer sets for use in absolute quantitation of copy number for 29 distinct genes of interest from the mouse (see Table 1). qPCR reactions consisted of primer pairs at a final concentration of $2.5 \mu \mathrm{M}$, cDNA template, and SsoAdvanced SYBR mastermix (BioRad) according to the manufacturer's instructions. Reactions were carried out on a CFXConnect (BioRad) machine with a three-step cycle of $95^{\circ} \mathrm{C}-15 \mathrm{~s}, 58^{\circ} \mathrm{C}-20 \mathrm{~s}, 72^{\circ} \mathrm{C}-20 \mathrm{~s}$, followed by a melt curve ramp from $65^{\circ} \mathrm{C}$ to $95^{\circ} \mathrm{C}$. Data were acquired during the $72^{\circ} \mathrm{C}$ step, and every $0.5^{\circ} \mathrm{C}$ of the melt curve. All reactions were run in triplicate, and the average $\mathrm{Ct}$ (cycle threshold) was used for interpolation with the standard curve to generate copy number for 
a given reaction. Standard curves for each gene were generated from a known copy number of a plasmid containing a partial ORF for a given gene of interest. Plasmids of known copy number were diluted from $10^{6}$ copies/ $\mu 1$ to 10 copies $/ \mu$ by factors of $10 \mathrm{x}$, and run in identical qPCR reactions to the samples. A line was fit to the standard curve and the subsequent equation for the fit line used to interpolate copy number from $\mathrm{Ct}$ values obtained from biological samples.

The units with which we express all of the qPCR data in this study is "normalized copy number per ng total RNA." All of the data are normalized relative to Glyceraldehyde 3-phosphate dehydrogenase (GAPDH) expression. A normalization factor using GAPDH for a sample $x$ was calculated by the following formula:

$$
\text { Normfactor }_{x}=\text { efficiency }_{G A P D H}-\left(C t_{x}-\text { AverageCt }_{G A P D H}\right)
$$

For our assay, GAPDH amplification efficiency was virtually $100 \%$, so this can be simplified as:

$$
\text { Normfactor }_{x}=2^{-\triangle C t_{G A P D H}}
$$

where $\Delta C t_{G A P D H}$ is the difference between the sample GAPDH and the population average, and 2 is the base of the exponential since $100 \%$ efficiency results in a doubling of product in each cycle of PCR. This resulted in the normalized copy number for a sample $x$ calculated as:

$$
\text { NormCopyNumber }_{x}=\frac{\text { RawCopyNumber }_{x}}{\text { Normfactor }_{x}}
$$

This normalization factor allows us to preserve the overall magnitude of the raw copy number, allowing for comparisons of mRNA abundance across genes. In addition, the normalization adjusts the copy number across different slices to account for differences in 
mRNA abundance that may occur for technical reasons, including variability in RNA extraction and reverse transcription efficiencies.

\begin{tabular}{|c|c|c|c|c|}
\hline Gene name & Accession \# & Gene Function & Forward primer (5' to 3') & Reverse Primer (5' to 3') \\
\hline CHRNA2 & NM_144803 & Nicotinic $\alpha 2$ & $\begin{array}{l}\text { TTATCTCTGGTGTCTGCTT } \\
\text { CTGA }\end{array}$ & CCCAGCGATTGTAGCCTCC \\
\hline CHRNA3 & NM_145129 & Nicotinic $\alpha 3$ & $\begin{array}{l}\text { TCCAGTTTGAGGTGTCTAT } \\
\text { GTCT }\end{array}$ & $\begin{array}{l}\text { TGGTAGTCAGAGGGTTTCCAT } \\
\text { TT }\end{array}$ \\
\hline CHRNA4 & NM_015730 & Nicotinic $\alpha 4$ & $\begin{array}{l}\text { CTAGCAGCCACATAGAGA } \\
\text { CCC }\end{array}$ & GACAAGCCAAAGCGGACAAG \\
\hline CHRNA5 & NM_176844 & Nicotinic $\alpha 5$ & $\begin{array}{l}\text { ATCCTCTGCTGCAAAACA } \\
\text { TGA }\end{array}$ & TCCACGTCCACTAACTGAGAT \\
\hline CHRNA6 & NM_021369 & Nicotinic $\alpha 6$ & $\begin{array}{l}\text { TAAAGGCAGTACAGGCTG } \\
\text { TGA }\end{array}$ & AAAATGCACCGTGACGGGAT \\
\hline CHRNA7 & NM_007390 & Nicotinic $\alpha 7$ & $\begin{array}{l}\text { CACATTCCACACCAACGT } \\
\text { CTT }\end{array}$ & $\begin{array}{l}\text { AAAAGGGAACCAGCGTACAT } \\
\text { C }\end{array}$ \\
\hline CHRNB2 & NM_009602.4 & Nicotinic $\beta 2$ & $\begin{array}{l}\text { ATTATCGCCTCACATGGA } \\
\text { AGC }\end{array}$ & $\begin{array}{l}\text { TGCCGTCAGCATTGTTGTATA } \\
\text { G }\end{array}$ \\
\hline CHRNB4 & NM_148944.4 & Nicotinic $\beta 4$ & $\begin{array}{l}\text { GGCAAGATCTACAGGAAG } \\
\text { CAT }\end{array}$ & ACACAATCACGAACACCCA \\
\hline CACNA1A & NM_007578 & $\begin{array}{l}\text { P/Q-type } \mathrm{Ca}^{2+} \\
\left(\mathrm{Ca}_{\mathrm{v}} 2.1\right)\end{array}$ & $\begin{array}{l}\text { AAAGGCTCCTACCTGAGG } \\
\text { AAT }\end{array}$ & CTCAGTGTCCGTAGGTCAAAC \\
\hline CACNA1B & NM_007579 & $\begin{array}{l}\text { N-type } \mathrm{Ca}^{2+} \\
\left(\mathrm{Ca}_{\mathrm{v}} 2.2\right)\end{array}$ & $\begin{array}{l}\text { ACAACGTCGTCCGCAAAT } \\
\text { AC }\end{array}$ & CAGGGCCAGAACAATGCAGT \\
\hline CACNA1C & NM_001159534 & $\begin{array}{l}\text { L-type } \mathrm{Ca}^{2+} \\
\left(\mathrm{Ca}_{\mathrm{v}} 1.2\right)\end{array}$ & $\begin{array}{l}\text { GGAGGGCGTGCATAAGCA } \\
\text { TT }\end{array}$ & $\begin{array}{l}\text { AGGAAGAGATACTCCACTCG } \\
\text { TTC }\end{array}$ \\
\hline$C A C N A 1 D$ & NM_001083616 & $\begin{array}{l}\text { L-type } \mathrm{Ca}^{2+} \\
\left(\mathrm{Ca}_{\mathrm{v}} 1.3\right)\end{array}$ & $\begin{array}{l}\text { GCTTACGTTAGGAATGGA } \\
\text { TGGAA }\end{array}$ & $\begin{array}{l}\text { GAAGTGGTCTTAACACTCGGA } \\
\text { AG }\end{array}$ \\
\hline CACNA1E & NM_009782 & $\begin{array}{l}\text { R-type } \mathrm{Ca}^{2+} \\
\left(\mathrm{Ca}_{v} 2.1\right)\end{array}$ & $\begin{array}{l}\text { CAGTCCGGCAGAACTGTT } \\
\text { TCA }\end{array}$ & $\begin{array}{l}\text { GAGACATTGGGGTCTTGTCAT } \\
\text { C }\end{array}$ \\
\hline CACNAIG & NM_001112813 & $\begin{array}{l}\text { T-type } \mathrm{Ca}^{2+} \\
\left(\mathrm{Ca}_{\mathrm{v}} 3.1\right)\end{array}$ & $\begin{array}{l}\text { AGAGTCCGCTGACCATGA } \\
\text { AAT }\end{array}$ & CCGGAATGTGTCGCAGATCA \\
\hline KCNA2 & NM_008417 & $\mathrm{K}_{\mathrm{V}} 1.2$ & $\begin{array}{l}\text { GTCTGAGCTTCTGCAGGA } \\
\text { AA }\end{array}$ & $\begin{array}{l}\text { CCTATTTGTGTATCTGTGCCA } \\
\text { TC }\end{array}$ \\
\hline KCNA3 & NM_008418 & $\mathrm{K}_{\mathrm{V}} 1.3$ & $\begin{array}{l}\text { CTGCCCATTACCTTGTCGT } \\
\mathrm{T}\end{array}$ & CAGTAAAGCCACCTTCTCCA \\
\hline KCNA4 & NM_021275 & $\mathrm{K}_{\mathrm{V}} 1.4$ & $\begin{array}{l}\text { GAAAGCAGGAAATGAAG } \\
\text { AGCATC }\end{array}$ & GTTGCAGCGTGGAAAAGG \\
\hline KCNA5 & NM_145983 & $\mathrm{K}_{\mathrm{V}} 1.5$ & $\begin{array}{l}\text { GCAGAGTCTCCAAGCAGA } \\
\text { AG }\end{array}$ & $\begin{array}{l}\text { TCTTCGAATACCCAGAAAGCT } \\
\text { C }\end{array}$ \\
\hline
\end{tabular}




\begin{tabular}{|l|l|l|l|l|}
\hline Gene name & Accession \# & Gene Function & Forward primer (5' to 3') & Reverse Primer (5' to 3') \\
\hline \hline KCNA6 & NM_013568 & $\mathrm{K}_{\mathrm{V}} 1.6$ & $\begin{array}{l}\text { CAGAGAAGTTCAAGATCG } \\
\text { GGTA }\end{array}$ & ATTTCTGCTTGGGATGAGGAC \\
\hline$K C N B 1$ & NM_008420 & $\mathrm{K}_{\mathrm{V}} 2.1$ & $\begin{array}{l}\text { GGTGGAGAGGACAATGA } \\
\text { ACA }\end{array}$ & GAGTTCGACAACACGTGCT \\
\hline$K C N B 2$ & NM_001098528 & $\mathrm{K}_{\mathrm{V}} 2.2$ & $\begin{array}{l}\text { AAGTGTGTTGAGAGACAG } \\
\text { AGC }\end{array}$ & TTGCTGGAGAAACCTAACTCG \\
\hline KCNC3 & NM_008422 & $\mathrm{K}_{\mathrm{V}} 3.3$ & CACAATGCTGCTCAGGCT & $\begin{array}{l}\text { GAAGACAAGAGCCCAATCAC } \\
\text { TCTB }\end{array}$ \\
\hline NM_007393 & Beta-actin & $\begin{array}{l}\text { GATGACCCAGATCATGTT } \\
\text { TGAGACC }\end{array}$ & $\begin{array}{l}\text { AGATGGGCACAGTGTGGGTG } \\
\text { A }\end{array}$ \\
\hline & NM_008084 & $\begin{array}{l}\text { G-phosphate } \\
\text { dehydrogenase }\end{array}$ & $\begin{array}{l}\text { TGCACCACCACCTGCTTA } \\
\text { GC }\end{array}$ & $\begin{array}{l}\text { GGCATGGACTGTGGTCATGA } \\
\text { G }\end{array}$ \\
\hline
\end{tabular}

Table 4.1 Gene identification and primer sets used for realtime PCR reactions

Accession numbers provided for each gene of interest. Primer sets were obtained from PrimerBank or generated de novo using PRIMER3 software. All primers sets were validated before use.

\subsubsection{1 $\underline{\text { Statistics }}$}

Data were analyzed using Sigmaplot 11.0 analysis software. Paired t-tests, Mann

Whitney-U tests, One-way repeated measures ANOVA test, and Friedman Repeated

Measure Analysis of Variance on Rank were used as appropriate. The data are presented as mean+SE.

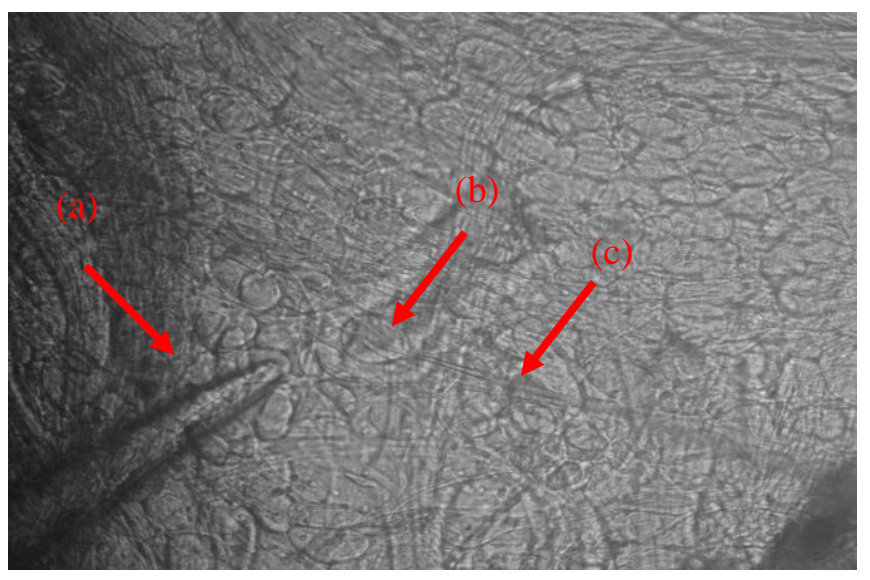

(A)

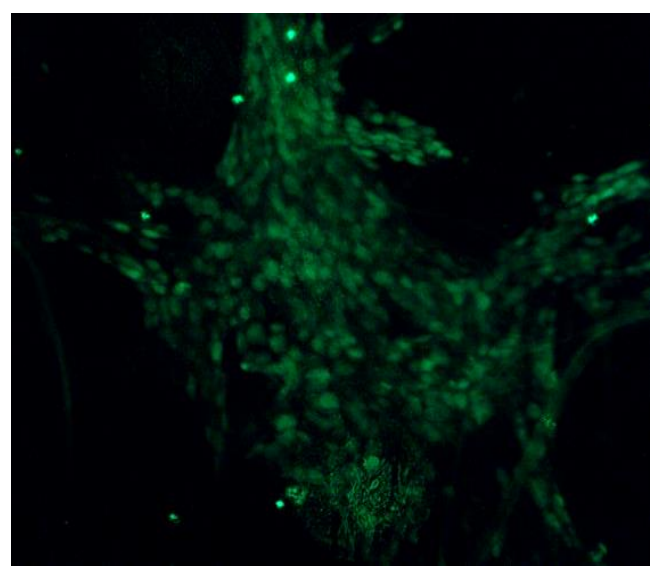

(B)

Figure 4.1 (A) Schematic showing the experimental set-up of ACh pressure ejection and intracellular recording electrode (a) Pressure-ejection electrode (b) Impaled cell (c) Intracellular recording electrode. (B) ChAT (BAC)-eGFP labelled parasympathetic neurons of MPG. 


\section{Results}

\section{Changes in post-synaptic currents in response to acetylcholine (ACh)}

Males

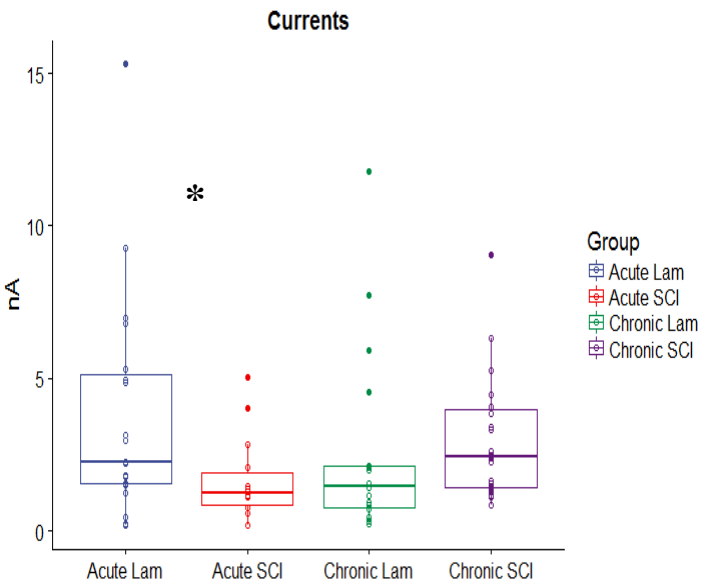

$\underline{\text { Females }}$

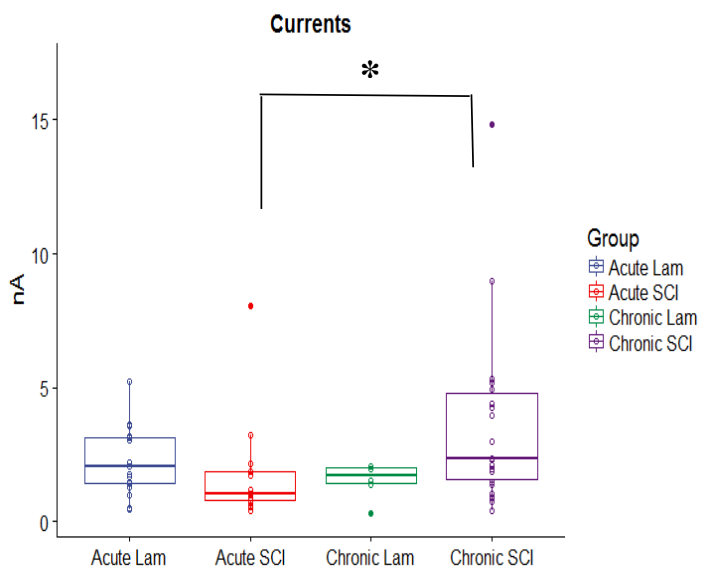

Figure 4.2 Synaptic strength of males were affected by acute SCI as well as with time after SCI whereas that of SCI females were not different compared to laminectomy groups.

A. Average current amplitudes of MPG neurons from males in acute and chronic SCI compared to laminectomy groups B. Average current amplitudes of MPG neurons from females in acute and chronic SCI compared to laminectomy groups. Data analyzed by Mann-Whitney U test $\left({ }^{*} \mathrm{p}<0.05\right.$, ${ }^{*} \mathrm{p}<0.01$, $* * * \mathrm{p} \leq 0.001)$.

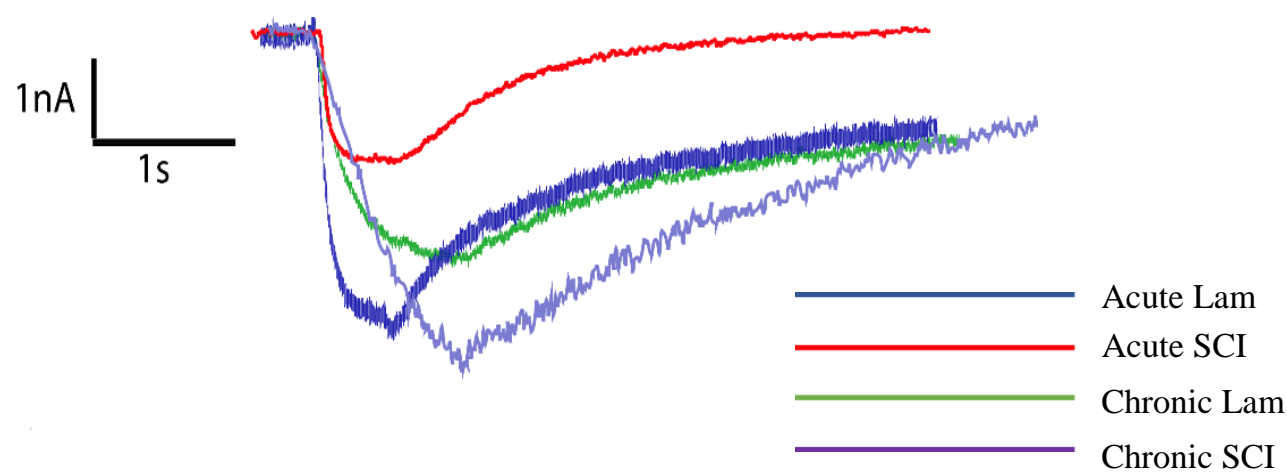

Trace 4.1 Representative current traces from ACh-evoked currents in 3-day vs. 28-day SCI and laminectomy groups. 
Our results showed decreased synaptic transmission of ACh-activated currents at acute SCI while relative increase in ACh-activated currents were observed at chronic SCI, and the currents were no longer different from their corresponding laminectomy values (Fig 4.2). We compared the four experimental groups in each sex separately at each time point by Mann-Whitney U tests. Our data showed significant differences between acute laminectomy and acute SCI groups in males $(\mathrm{P}=0.030)$ and but not in females $(\mathrm{P}=0.056)$

(Fig 4.2). At chronic SCI, the current amplitudes were not different between laminectomy and SCI groups for both males $(\mathrm{P}=0.064)$ and females $(\mathrm{P}=0.356)$ (Fig 4.2). Significant differences between acute and chronic SCI groups were observed in both males $(\mathrm{P}=0.012)$, and in females $(\mathrm{P}=0.016)($ Fig 4.2). There were no statistical differences between 3-day and 1-month laminectomy groups for both males $(\mathrm{P}=0.162)$ and females $(\mathrm{P}=0.575)$.

\section{Changes in intrinsic excitability of MPG neurons}

(A)

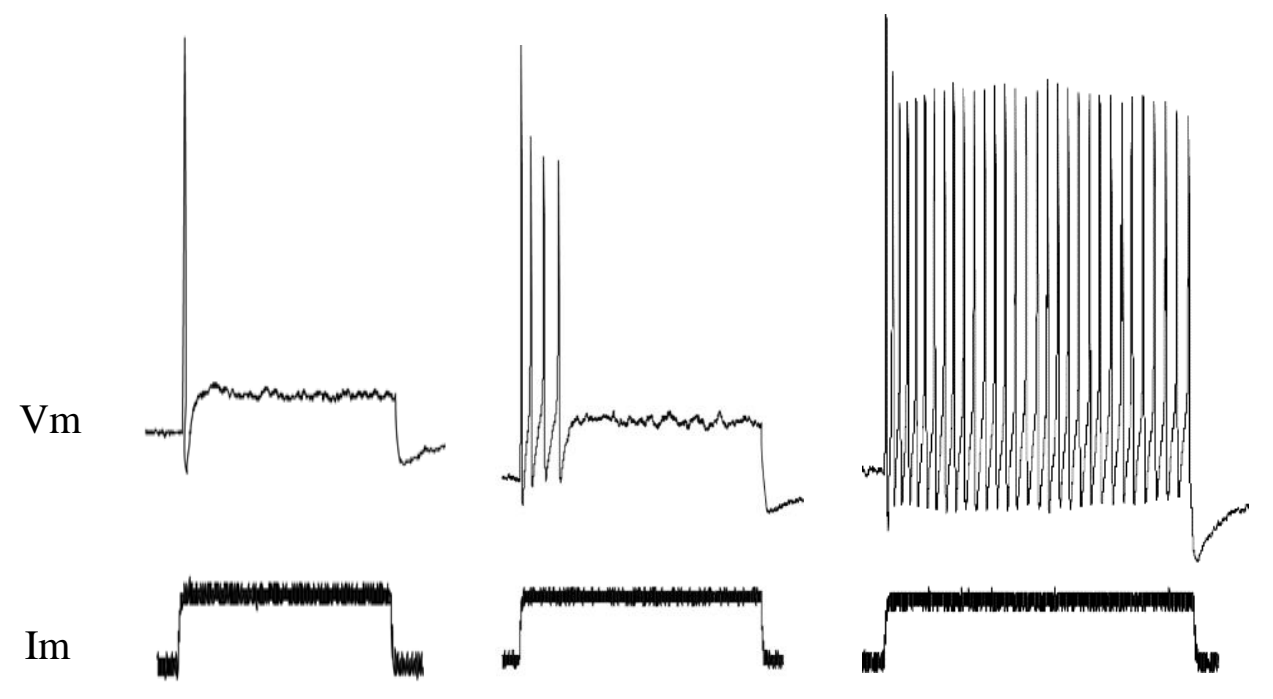

Trace 4.2 Voltage traces in response to current injections 
(B)
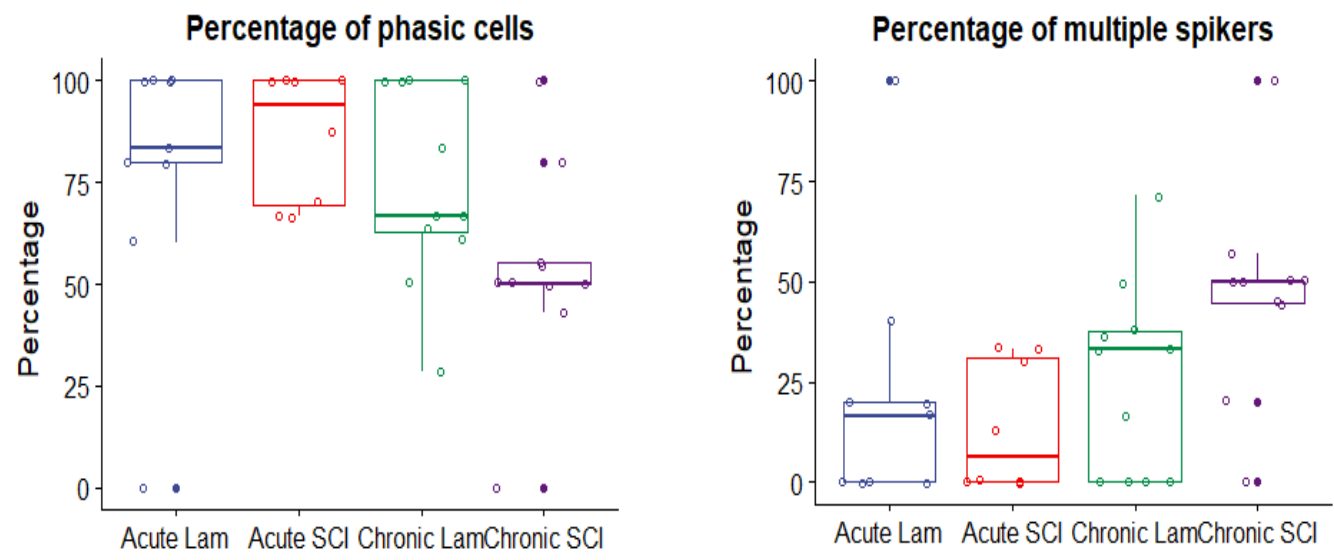

Figure 4.3 Percentage of phasic cells decreased considerably in chronic SCI MPG.

(A). 3-types of firing patterns: phasic (i), intermediate (ii), and tonic (iii), were observed in response to current injection. (B). Percentage of cells that responded with phasic spikes was observed to be decreased in chronic SCI group while the percentage of multiple spikers was significantly increased.

In response to intracellular current injection for duration $\sim 500 \mathrm{~ms}$, (3) types of responses:

phasic (single spikers), intermediate (slowly-adapting multiple spikers), and tonic (nonadapting) firing patterns were observed (Trace 4.2). 
(A) Males
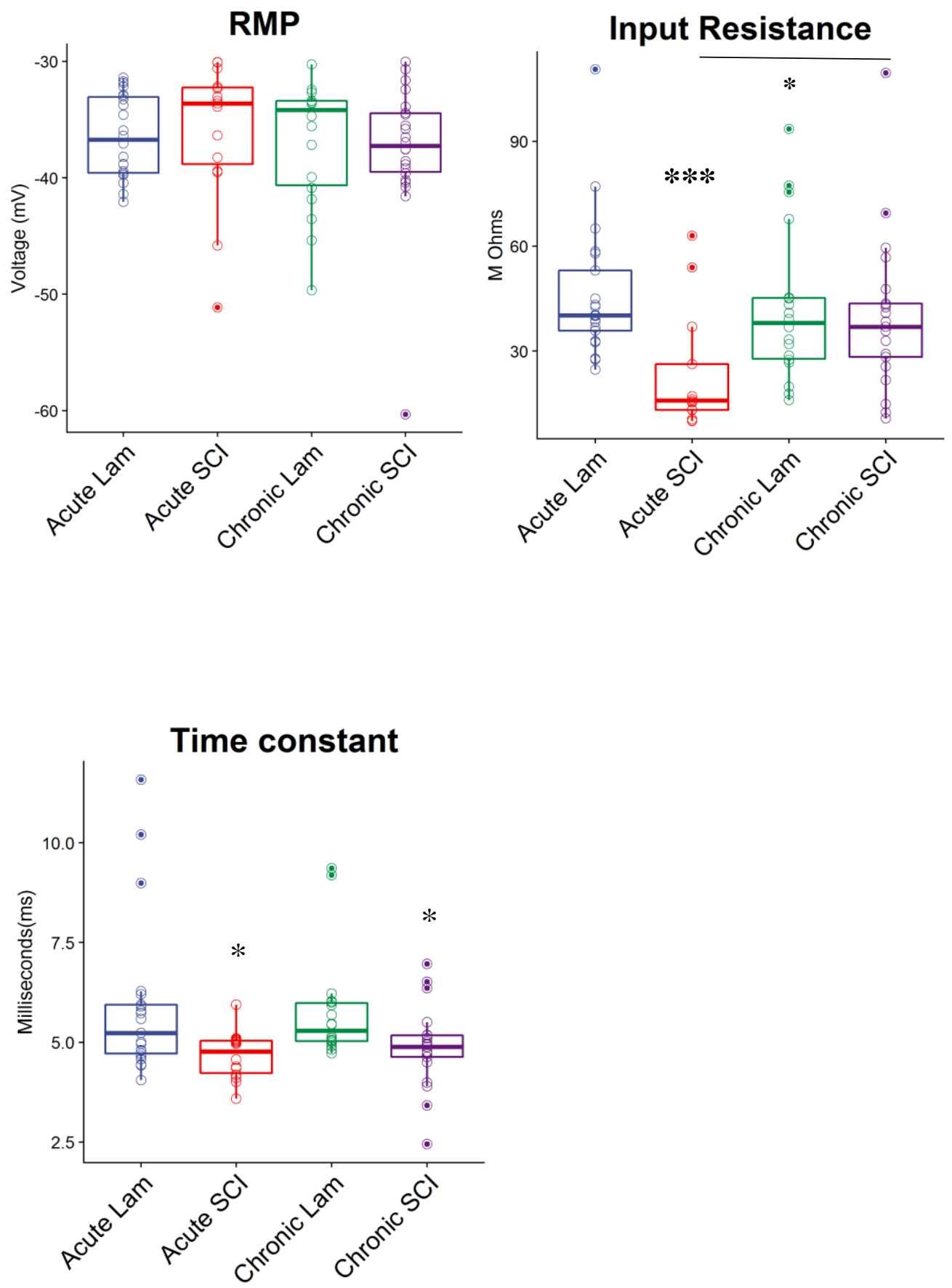
(B) Females

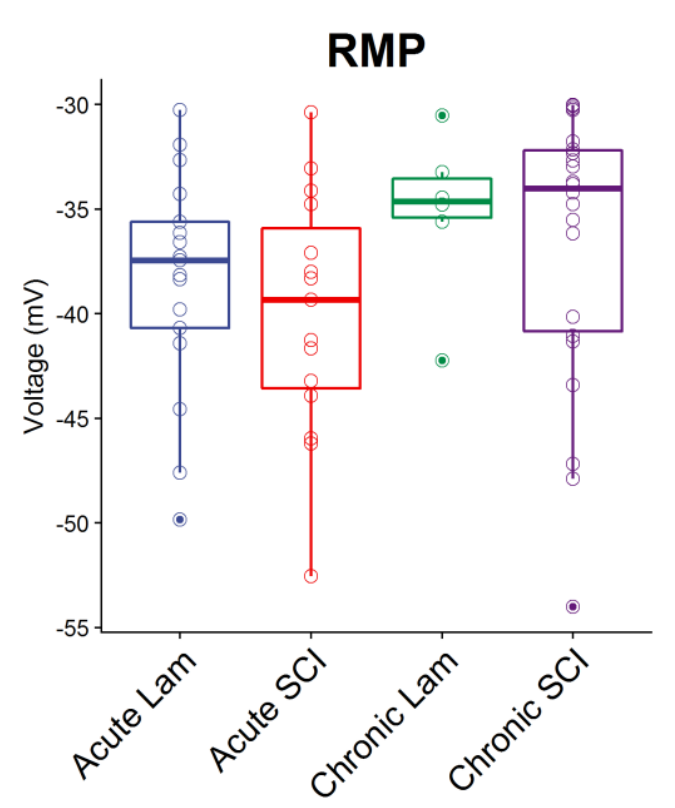

\section{Input Resistance}

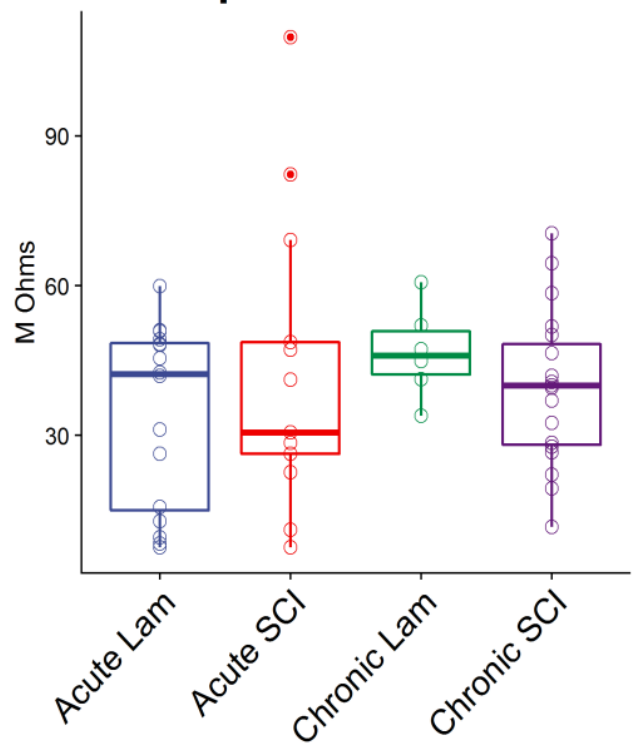

Time constant

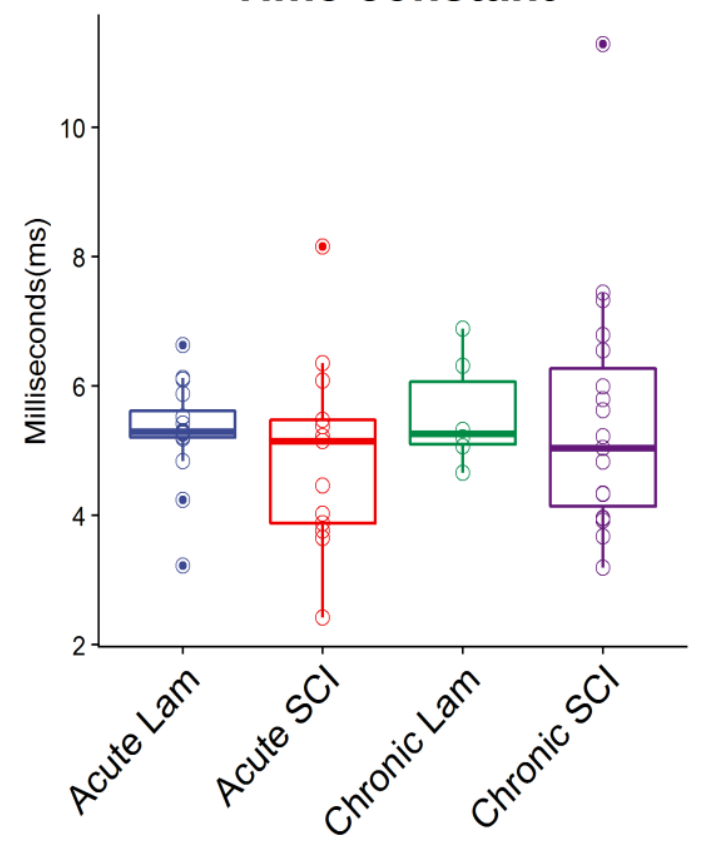

Figure 4.4 Intrinsic properties of MPG neurons in females did not show significant changes between laminectomy and spinal cord injury groups.

A. Males- acute lam $n=22$, acute $S C I n=15$, chronic lam $n=18$, chronic $S C I n=22$.B. Females acute lam $n=16$, acute $S C I n=15$, chronic lam $n=6$, chronic $S C I n=22$. (Unpaired t-test and

Mann-Whitney Rank Sum Tests were used as appropriate- ${ }^{*} \mathrm{p}<0.05,{ }^{* *} \mathrm{p}<0.01,{ }^{* * *} \mathrm{p} \leq 0.001$ ) 
Next, we explored if the intrinsic properties of the neurons in laminectomy groups and SCI groups at both time periods. Resting membrane potential (RMP) among all four groups were not different in both males and females. Input resistances were measured by dividing the change in voltage by corresponding amplitude of hyperpolarizing current injections. Time constants were measured by single exponential fittings of the hyperpolarized voltage traces in response to hyperpolarizing current injections. Within females, no significant differences were observed in input resistances or time constants. Within males, acute SCI group had significantly lower input resistance compared to those of acute laminectomy and chronic SCI ( $\mathrm{P}=<0.001)$ (Fig 4.4 A). Chronic SCI group was not significantly different from that of laminectomy group. Both acute SCI and chronic SCI groups had lower time constants compared to their respective laminectomy groups ( $\mathrm{P}=0.025$ for acute, $\mathrm{P}=0.013$ for chronic) (Fig $4.4 \mathrm{~A})$. 
4.2.12 Changes in mRNA expressions of nAChRs and voltage-gated ion channels

\begin{tabular}{|c|c|c|}
\hline \multirow[t]{2}{*}{ Genes } & $\begin{array}{c}\text { Acute laminectomy } \\
\text { vs SCI } \\
\end{array}$ & $\begin{array}{c}\text { Chronic laminectomy } \\
\text { vs. SCI } \\
\end{array}$ \\
\hline & $\mathrm{P}$ value & $\mathrm{P}$ value \\
\hline $\mathrm{ACHE}$ & 0.638 & 0.341 \\
\hline ChAT & 0.754 & $0.00023 * * *$ \\
\hline CHRNA2 & 0.19 & 0.82 \\
\hline CHRNA3 & $0.0168 *$ & 0.0811 \\
\hline CHRNA4 & 0.25 & 0.3 \\
\hline CHRNA5 & 0.49 & 0.83 \\
\hline CHRNA6 & 0.37 & 0.61 \\
\hline CHRNA7 & 0.62 & 0.8 \\
\hline CHRNB2 & 0.575 & 0.093 \\
\hline CHRNB4 & 0.8816 & $0.0053 * *$ \\
\hline CACNA1A & 0.627 & 0.062 \\
\hline CACNA1B & 0.751 & 0.036 \\
\hline CACNA1C & 0.4 & 0.39 \\
\hline CACNA1D & 0.363 & $0.042 *$ \\
\hline CACNA1E & 0.38 & 0.78 \\
\hline CACNA1G & 0.61 & 0.57 \\
\hline CACNA1I & 0.42 & 0.8 \\
\hline KCNA2 & 0.57 & 0.28 \\
\hline KCNA3 & 0.57 & 0.36 \\
\hline KCNA4 & 0.916 & $0.035^{*}$ \\
\hline KCNA5 & 0.93 & 0.66 \\
\hline KCNA6 & 0.246 & 0.058 \\
\hline KCNB1 & 0.68 & 0.33 \\
\hline $\mathrm{KCNB} 2$ & 0.44 & 0.16 \\
\hline KCNC1 & 0.71 & 0.26 \\
\hline $\mathrm{KCNC} 2$ & 0.76 & 0.53 \\
\hline KCNC3 & 0.38 & 0.72 \\
\hline $\mathrm{KCNC} 4$ & 0.586 & 0.103 \\
\hline $\mathrm{KCND} 2$ & 0.91 & 0.87 \\
\hline KCNMA1 & 0.483 & 0.089 \\
\hline SCN2A1 & 0.25 & 0.48 \\
\hline SCN3A & 0.704 & $0.013^{*}$ \\
\hline SCN8A & 0.48 & 0.84 \\
\hline Actin & 0.38 & 0.35 \\
\hline
\end{tabular}

Table 4.2 Table showing P-values for comparison of mRNA transcripts between acute laminectomy and acute SCI, and chronic laminectomy and chronic SCI groups using Dunn's comparison tests. 

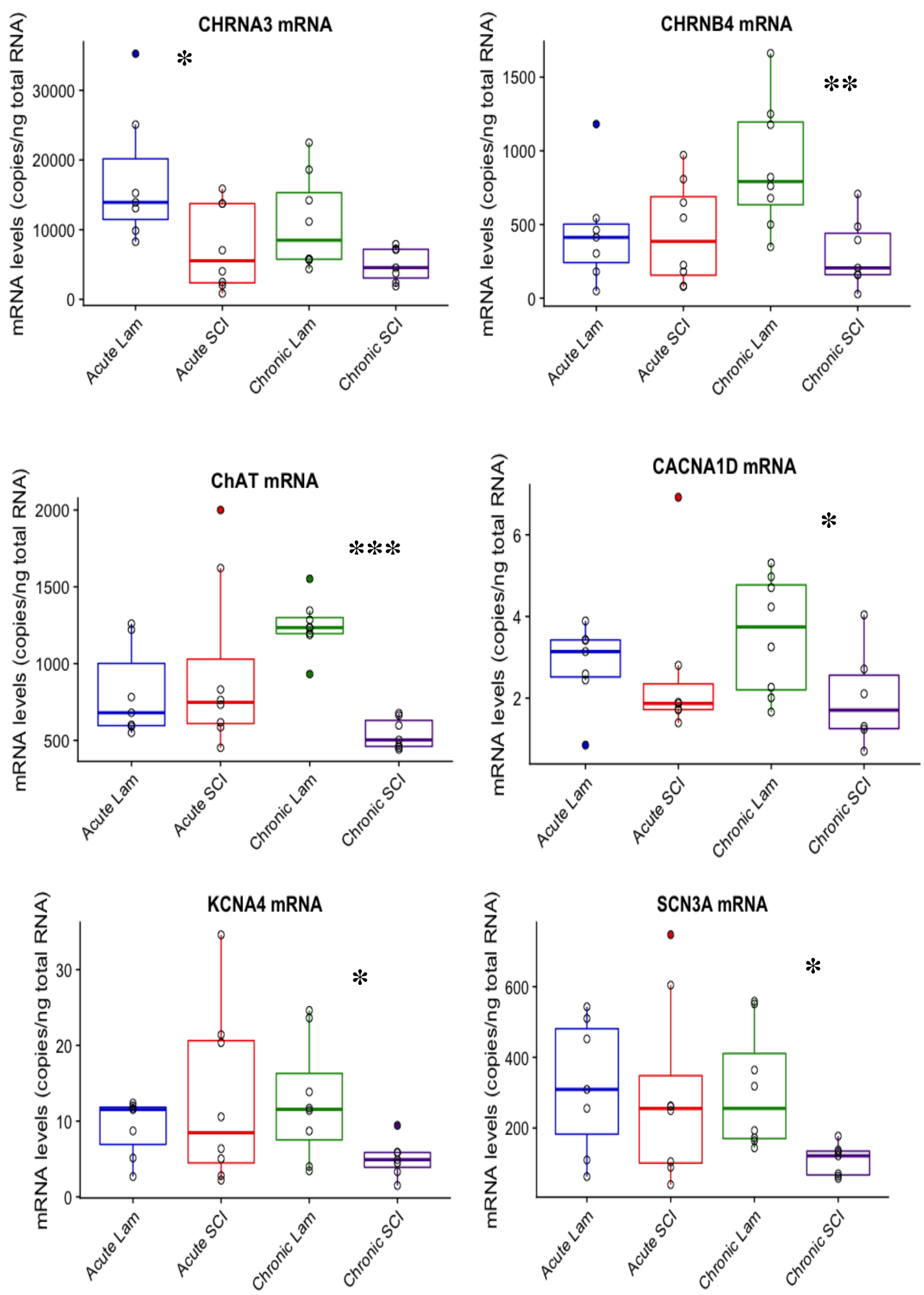

Figure 4.5 Significant changes in expression levels of mRNA transcripts for nicotinic receptor $(\mathrm{nAChR})$ subunits and voltage-gated calcium, potassium and sodium channels were observed from RT-PCR analyses. 
Analysis of mRNA abundances showed that major nicotinic receptor subunit ( $\alpha 3)$ was downregulated at acute SCI $(\mathrm{P}=0.017)$ (Fig 4.5). At chronic SCI, $\alpha 3$ receptor abundances were upregulated back to laminectomy levels, however, significant reduction of $\beta 2$ subunit $(\mathrm{P}=0.005)$ and ChAT $(\mathrm{P}=0.00023)$ was observed (Fig 4.5). Downregulation voltage-gated "L-type" calcium channel (CACNA 1d) (P=0.042), “A-type” potassium channel (KCNA 4), and sodium channel (SCN3A) also emerged at chronic SCI (P= 0.035) (Fig 4.5), however, it should be noted their mRNA expression levels were very low. We did not see any significant differences in mRNA expressions for other nicotinic receptor subunits or other voltage-gated ion channels (Table 4.2).

\subsubsection{Action potential properties}

Action potential properties of the MPG neurons were measured from the rebound action potentials in responses to hyperpolarizing current injections of $(-400) \mathrm{pA}$. Threshold to fire action potentials were measured at the voltage at which $10 \mathrm{mV} / \mathrm{ms}$ point on the rising slope of rebound action potentials were observed. Properties of the rebound action potentials were measured by selecting each action potential and statistics were generated by using 'Analyze' tool in Clampfit 10.7 analysis software. 
(A) Males
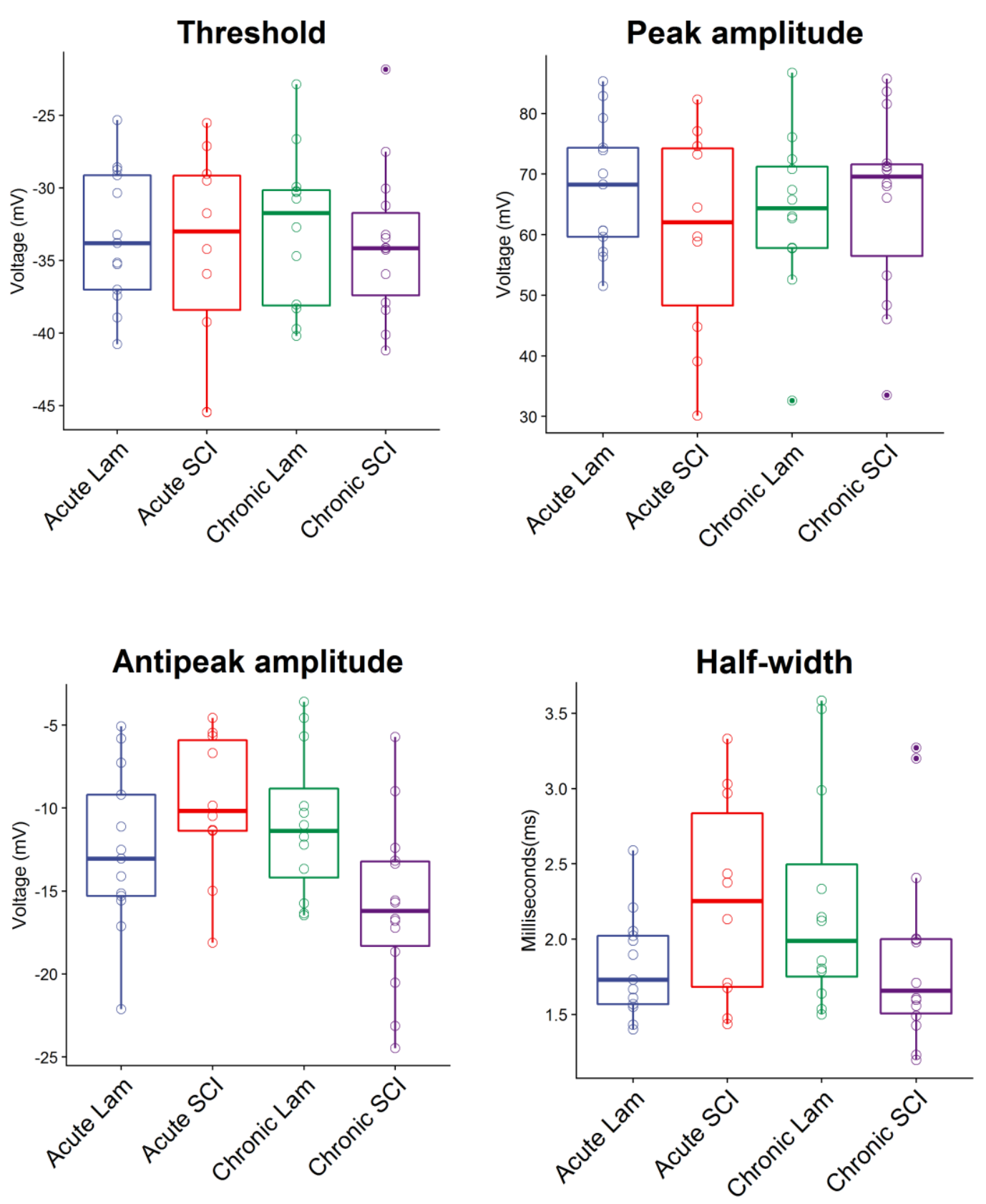

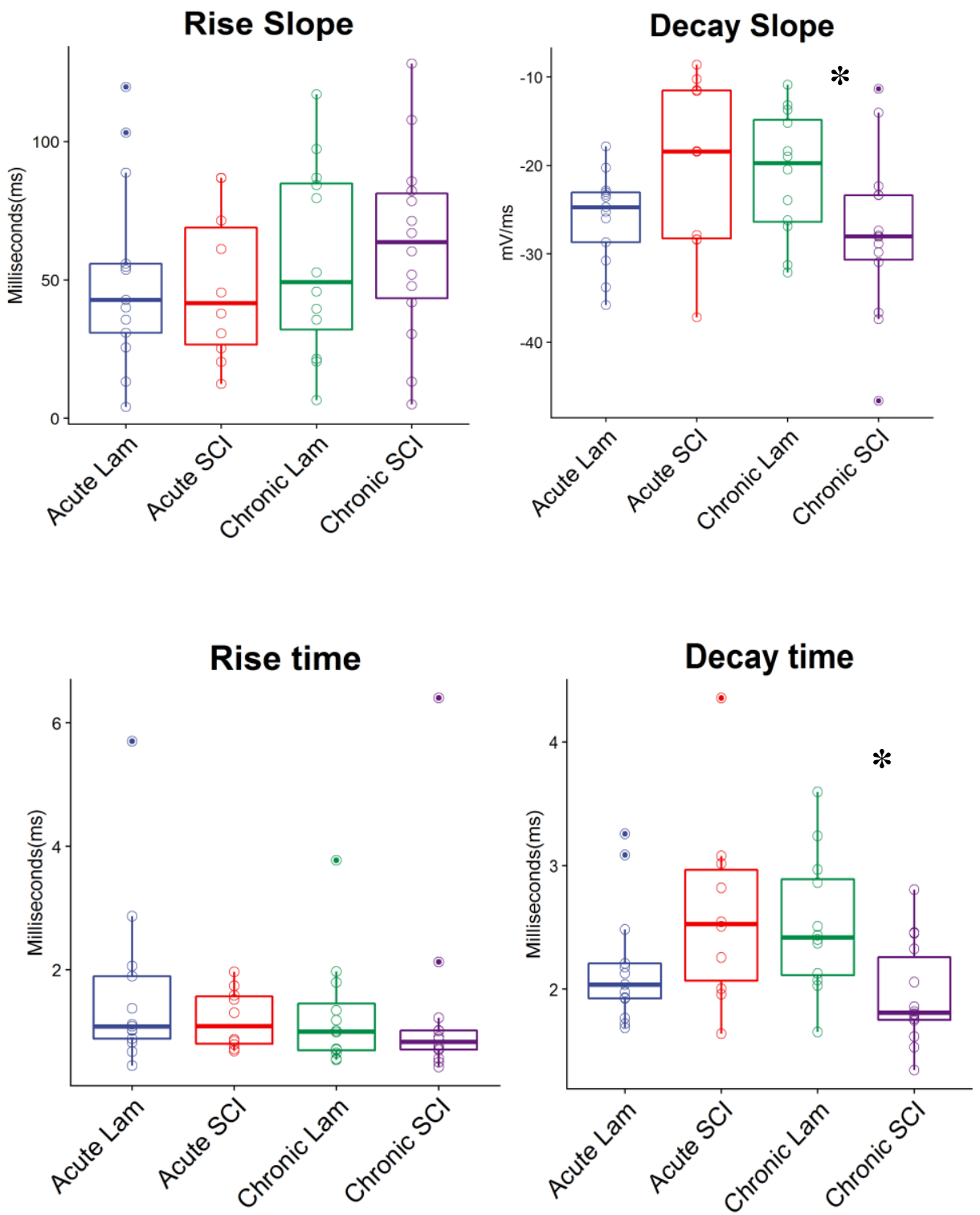

Figure 4.6 Significant changes in action potential properties associated with narrower action potentials were observed in males at chronic SCI.

(Unpaired t-test and Mann-Whitney Rank Sum Tests were used as appropriate$* \mathrm{p}<0.05, * * \mathrm{p}<0.01, * * * \mathrm{p} \leq 0.001$ ) (Males- acute lam $\mathrm{n}=13$, acute SCI $\mathrm{n}=10$, chronic lam $n=11$, chronic SCI $n=14)$. 
Within males, antipeak amplitude (AHP) $(\mathrm{P}=0.015$ (chronic lam vs. SCI), $\mathrm{P}=0.004$ (acute vs. chronic $\mathrm{SCI})$, decay slope and $(\mathrm{P}=0.047)$ decay time $(\mathrm{P}=0.006$ (chronic lam vs. $\mathrm{SCI})$, $\mathrm{P}=0.005$ (acute vs. chronic SCI)), were significantly different between chronic laminectomy and SCI groups (Fig 4.6 A). No significant differences were found among groups for threshold, peak amplitude, half-width, rise slope, and rise time.

(B) Females
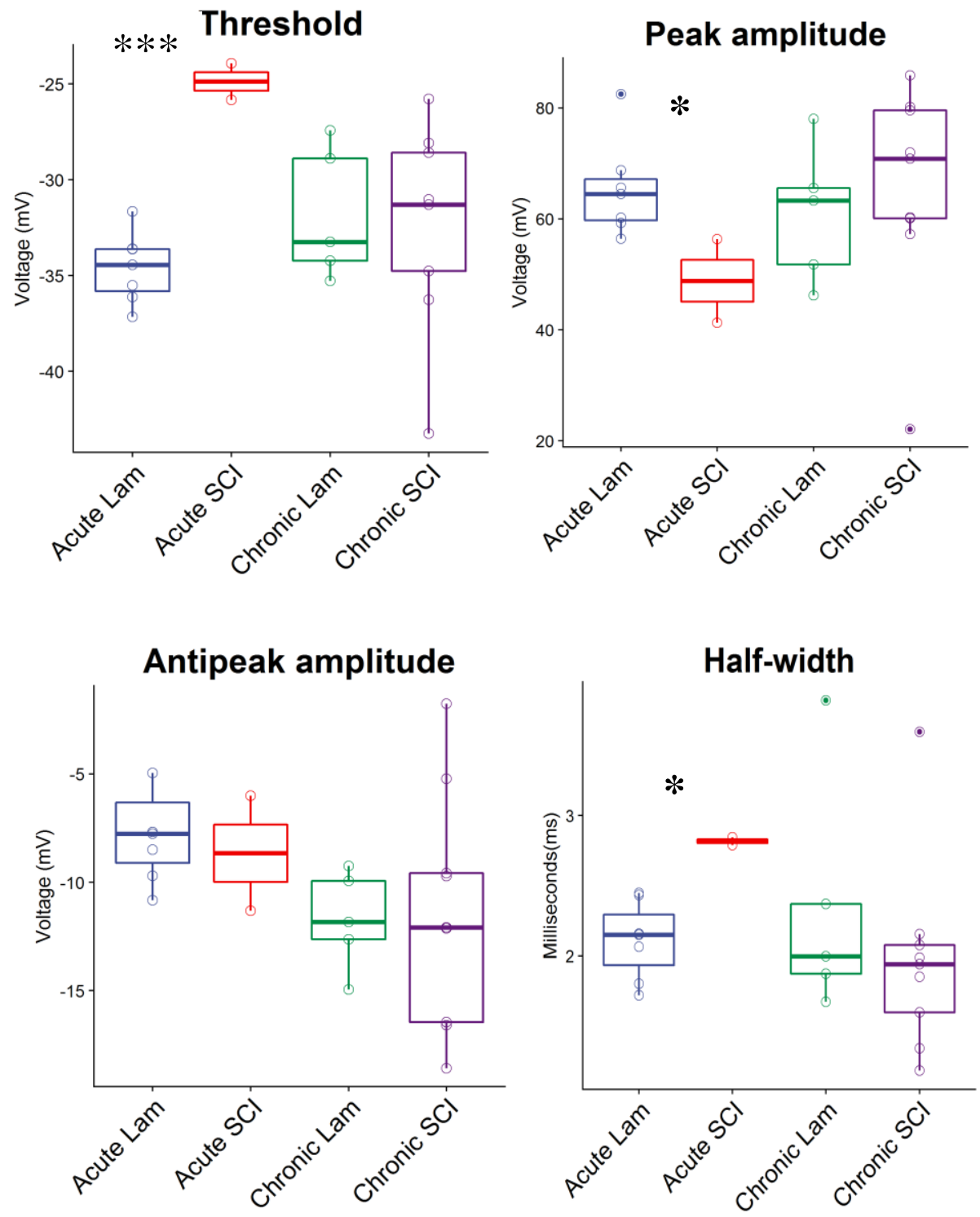

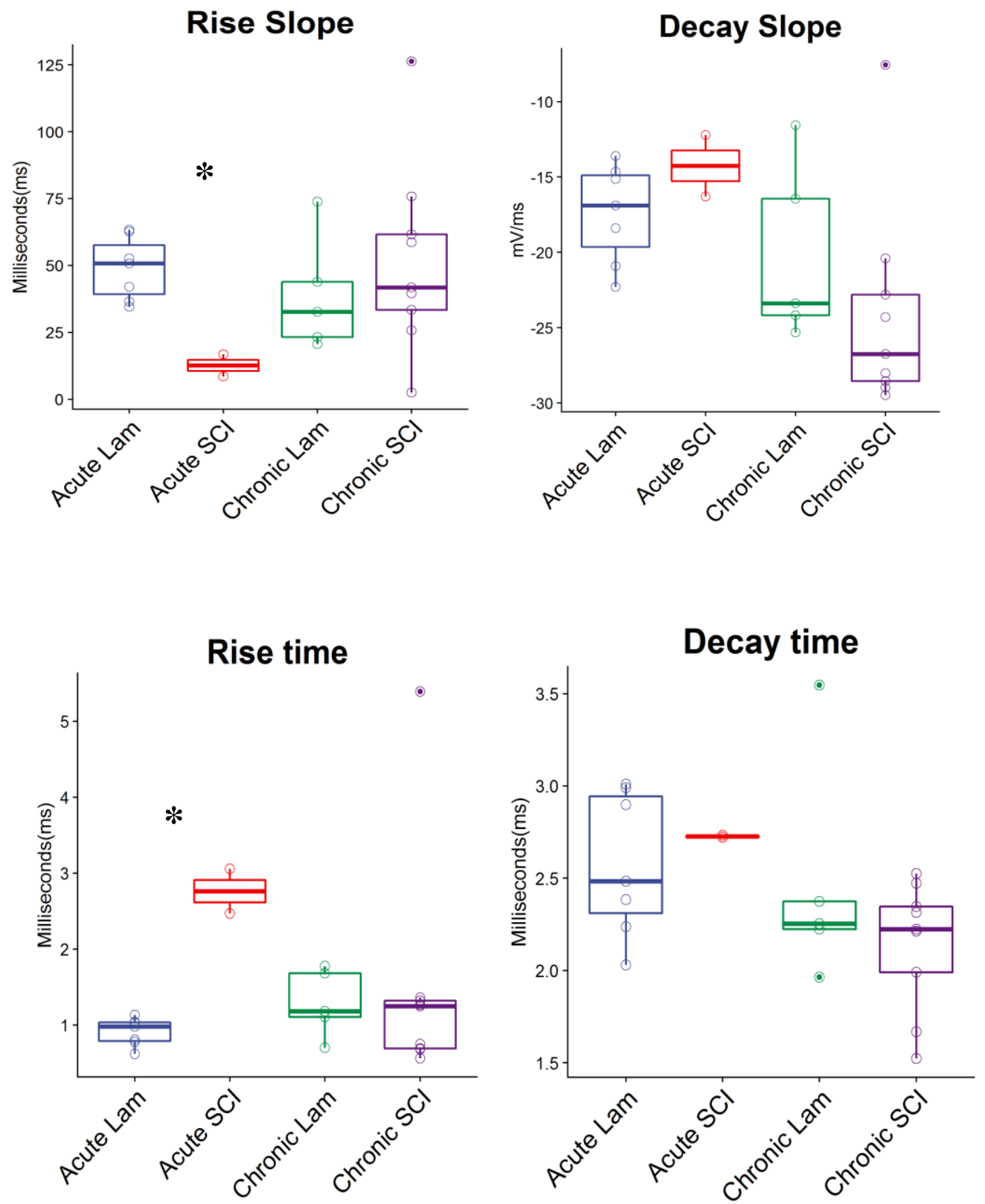

Figure 4.7 Significant changes in action potential properties associated with narrower action potentials were observed at acute SCI and with time post-injury in females.

(Unpaired t-test and Mann-Whitney Rank Sum Tests were used as appropriate${ }^{*} \mathrm{p}<0.05, * * \mathrm{p}<0.01, * * * \mathrm{p} \leq 0.001$ ) (Females - acute lam $\mathrm{n}=7$, acute SCI $\mathrm{n}=2$, chronic lam $n=6$, chronic SCI $n=9)$. 
Within females, acute SCI had significantly higher threshold than laminectomy $(\mathrm{P}=<0.001)$ with lower peak amplitudes $(\mathrm{P}=0.048)$ (Fig.6 B). The half-width of acute SCI was also longer than that of acute laminectomy $(\mathrm{P}=0.022)$ (Fig 4.7). Acute SCI groups had smaller rise slope $(\mathrm{P}=0.010)$ and longer duration of rise time $(\mathrm{P}=<0.001)$ compared to the laminectomy group (Fig 4.7). The mean decay time was not significantly different between 3-day laminectomy and SCI groups but the 3-day SCI group had significantly longer mean decay time compared to that of chronic SCI group ( $\mathrm{P}=0.049)$ (Fig 4.7). Membrane properties (Input resistance and Time constant) were decreased at acute SCI. However, at chronic injury stage, action potential properties (Antipeak amplitude, Decay slope, and Decay time) were changing in such a way that action potential have faster slopes with shorter durations and increased afterhyperpolarization (AHP) (antipeak) amplitudes resulting in narrow and high action potentials. Within the injury groups, there are time-dependent changes in membrane, synaptic (Current density, Currents), and action potential properties (Antipeak amplitude, and Decay time). The changes in membrane and synaptic properties seen at acute stage recovered in chronic SCI, however, the changes in action potential properties seen at chronic stage seem to be changing towards longer-amplitude action potentials with narrower durations. In females, at acute stage, none of the membrane and synaptic properties are changing however, the action potential properties (Threshold, Peak amplitude, Half-width, Rise slope, Rise time) are changing in such a way that the action potentials have more depolarized threshold, shorter peaks and longer durations with smaller rise slopes and longer rise times leading to wider and shorter action potentials. However, at chronic stage, all the changes seen at acute stage are no longer significant. They changed in such 
a way the changes seen at acute stage fully recovered without overshooting. All of the values revert back to values similar to laminectomy values. Within injury groups, the only values that remain significantly altered with time are currents, Max rise slope, and Decay time but they were all back to laminectomy values at chronic stage.

\subsubsection{Response of the MPG neurons to pelvic nerve stimulation after SCI}

In response to pelvic nerve stimulation, some of the MPG neurons responded with suprathreshold responses or action potentials (APs) while others responded with subthreshold responses or excitatory postsynaptic responses (EPSPs). The percentages of cells that responded with action potentials and subthreshold responses are, $70 \%(14 / 20)$ APs and 30\% (6/20) EPSPs in acute laminectomy, 33.34\% (2/6) APs and 66.67\% (4/6) EPSPs in acute SCI, $12.5 \%$ (2/16) APs and 87.5\% (14/16) EPSPs in chronic laminectomy, and 45.83\% (11/24) APs and 54.17\% (13/24) EPSPs in chronic SCI groups. We performed experiments by stimulating the pelvic nerve at various frequencies to measure frequency-dependence of synaptic responses in EPSP generating neurons, and to measure frequency ranges that can reliably generate action potentials in AP generating neurons. 
3-day EPSPs at $1 \mathrm{~Hz}$

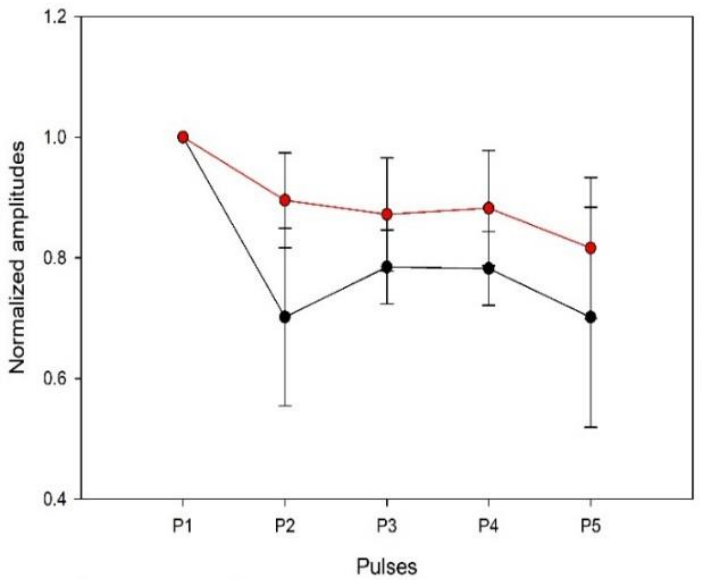

$$
\begin{aligned}
& \bullet \text { 3-day Lam } \\
& - \text { 3-day SCI }
\end{aligned}
$$

3-day EPSPs at $2 \mathrm{~Hz}$

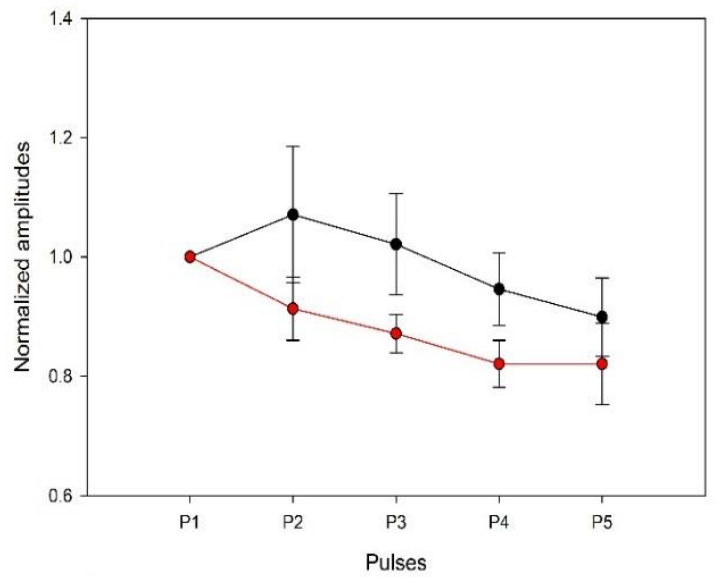

3-day EPSPs at $5 \mathrm{~Hz}$

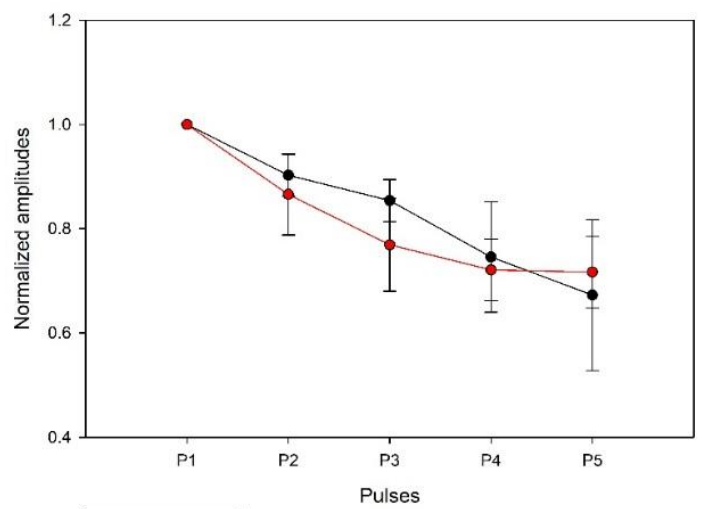

- 3-day Lam

3-day SCI 1-month EPSPs at $1 \mathrm{~Hz}$

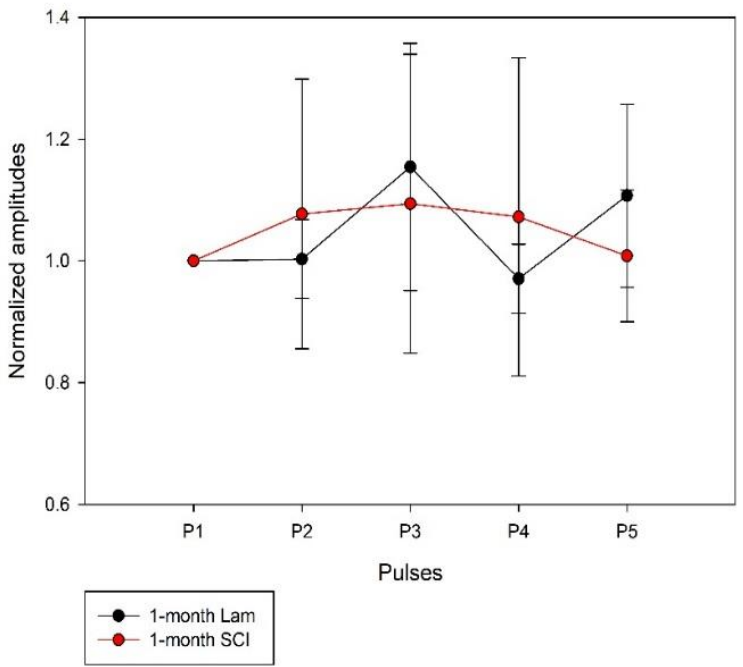

1-month EPSPs at $2 \mathrm{~Hz}$
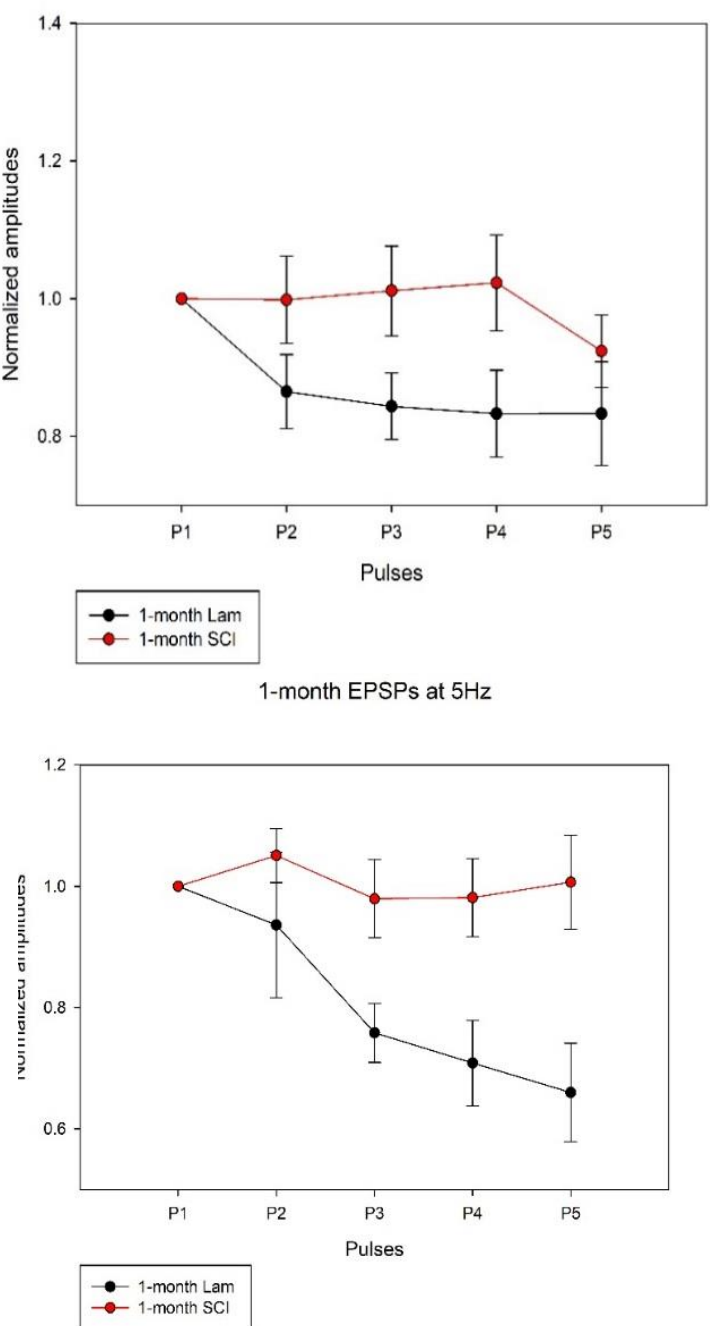

111 

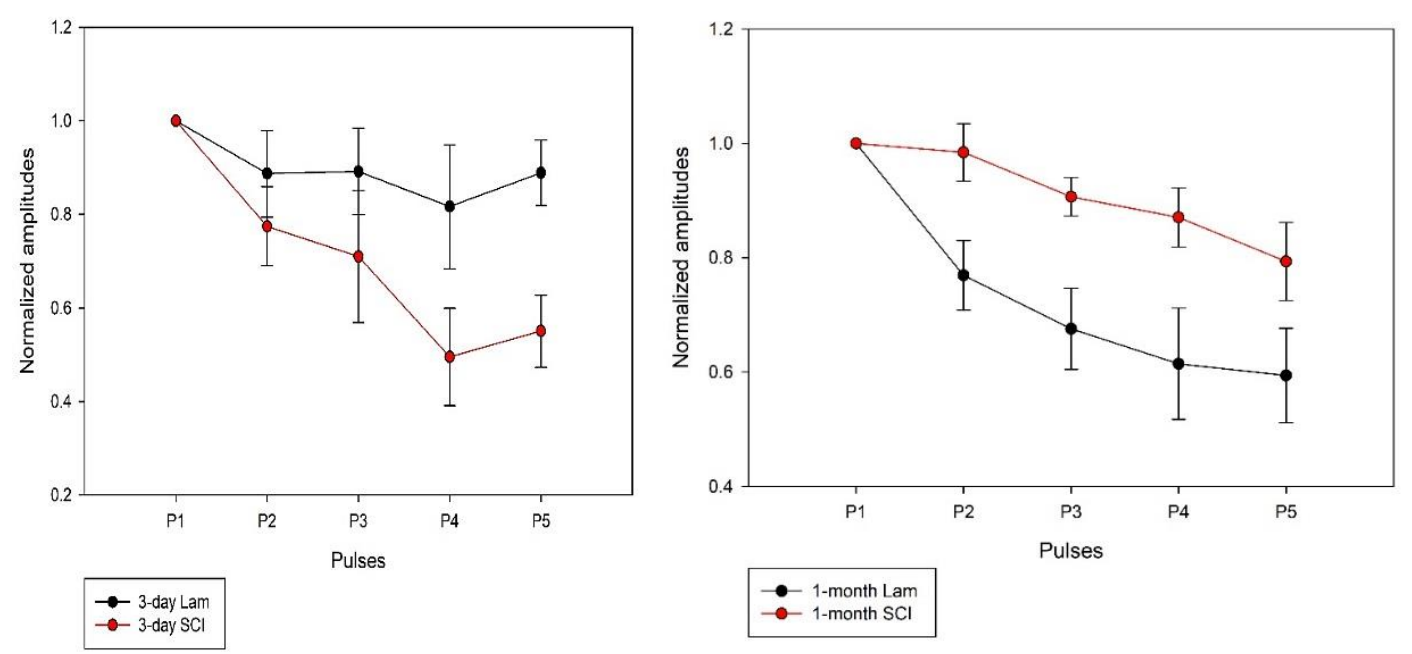

3-day EPSPs at $20 \mathrm{~Hz}$
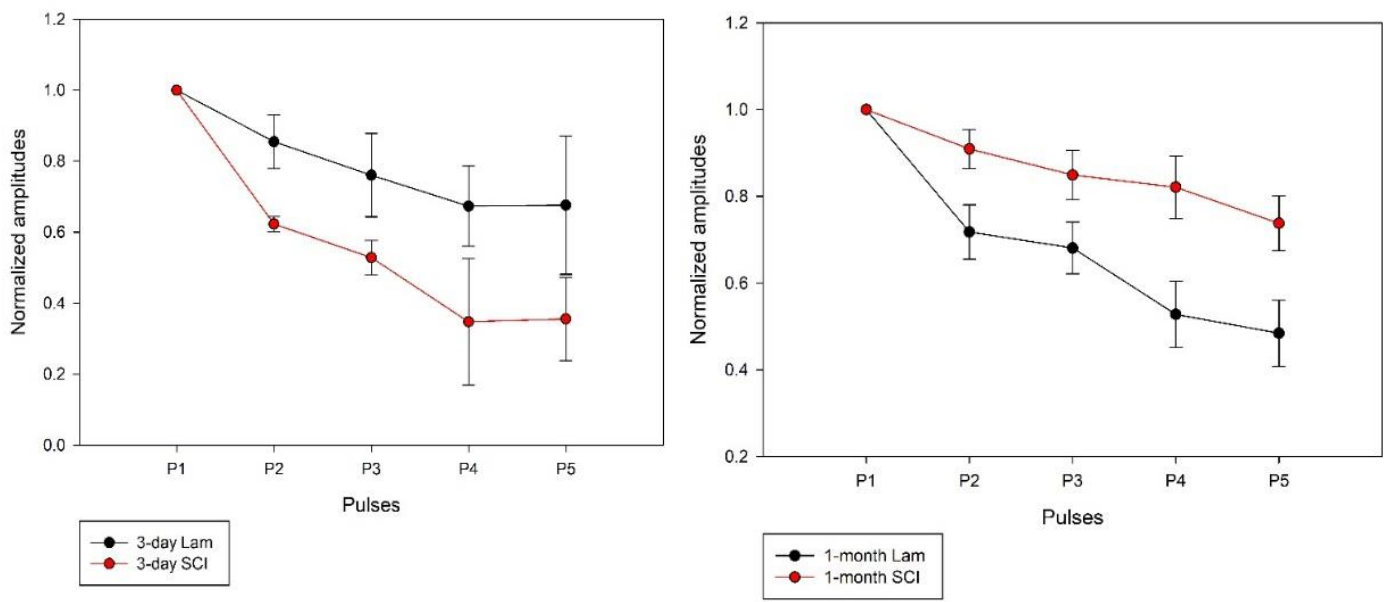

Figure 4.8 Depression of EPSPs were observed at higher frequencies of stimulations in both laminectomy groups and 3-day SCI, however, 1-month SCI group did not show significant depression of EPSPs.

Pelvic nerve was stimulated at $1 \mathrm{~Hz}, 2 \mathrm{~Hz}, 5 \mathrm{~Hz}, 10 \mathrm{~Hz}$, and $20 \mathrm{~Hz}$ frequencies and the evoked postsynaptic depolarizations were measured in current clamp mode.The amplitudes of the responses were normalized to the first pulse. The normalized amplitudes from the 1 st pulse to the 5 th pulse at each frequency are shown as mean + SE for each experimental group. (3-day Lam n $1 \mathrm{~Hz}=6,2 \mathrm{~Hz}=5,5 \mathrm{~Hz}=4,10 \mathrm{~Hz}=5,20 \mathrm{~Hz}=4$; 3-day $\mathrm{SCI}$ n $1 \mathrm{~Hz}$ $=4,2 \mathrm{~Hz}=4,5 \mathrm{~Hz}=4,10 \mathrm{~Hz}=3,20 \mathrm{~Hz}=2 ; 1$-month $\mathrm{Lam} \mathrm{n} 1 \mathrm{~Hz}=14,2 \mathrm{~Hz}=14,5 \mathrm{~Hz}=14$, $10 \mathrm{~Hz}=14,20 \mathrm{~Hz}=12,1$-month SCI n $1 \mathrm{~Hz}=8,2 \mathrm{~Hz}=12,5 \mathrm{~Hz}=13,10 \mathrm{~Hz}=11,20 \mathrm{~Hz}=10$ ). 

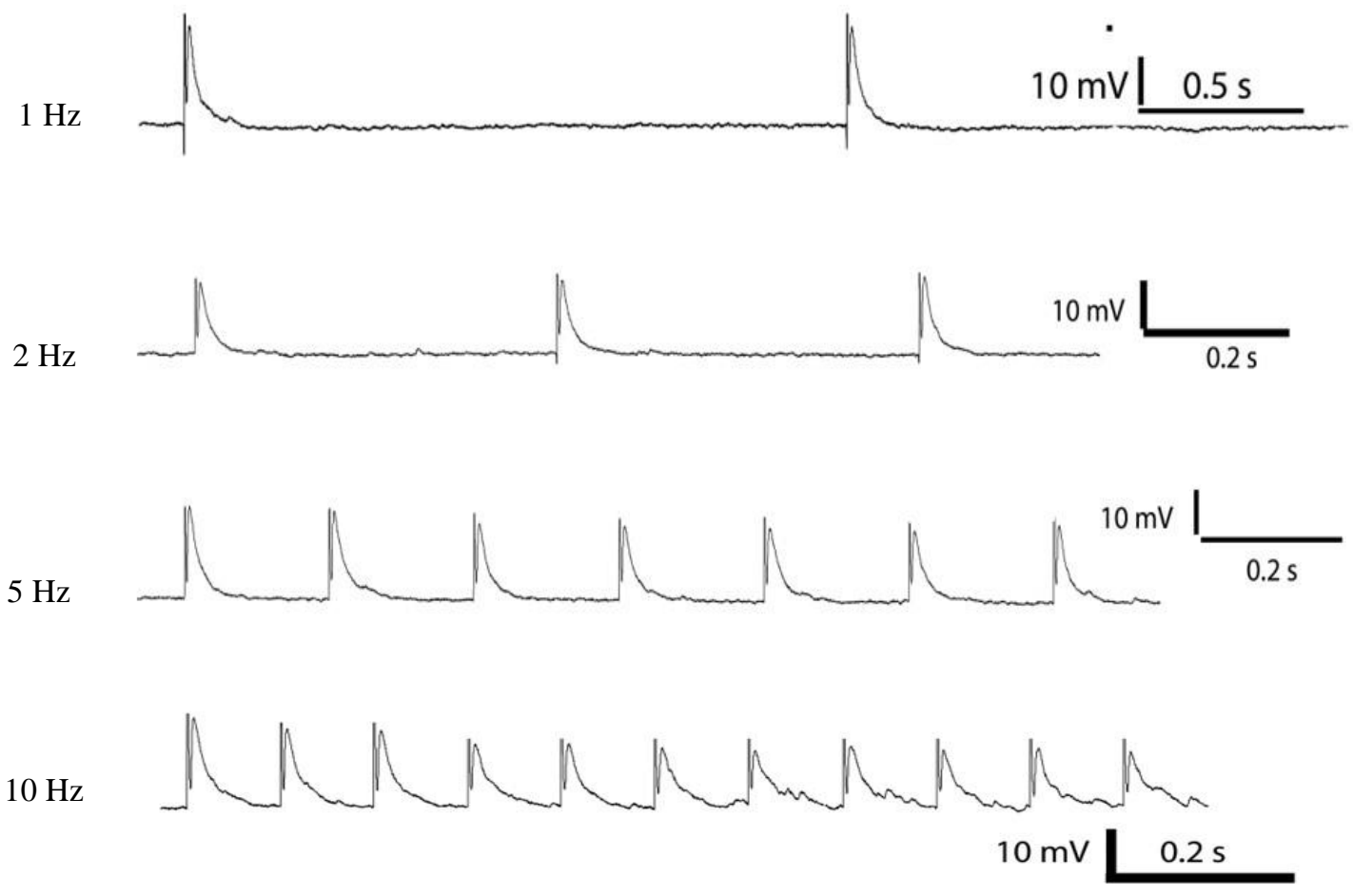

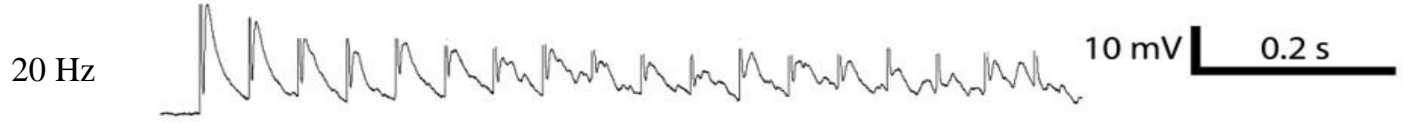

Trace 4.3 Example traces of pelvic-nerve stimulated EPSPs at $1 \mathrm{~Hz}, 2 \mathrm{~Hz}, 5 \mathrm{~Hz}, 10$ $\mathrm{Hz}$ and $20 \mathrm{~Hz}$ in 3-day laminectomy. 

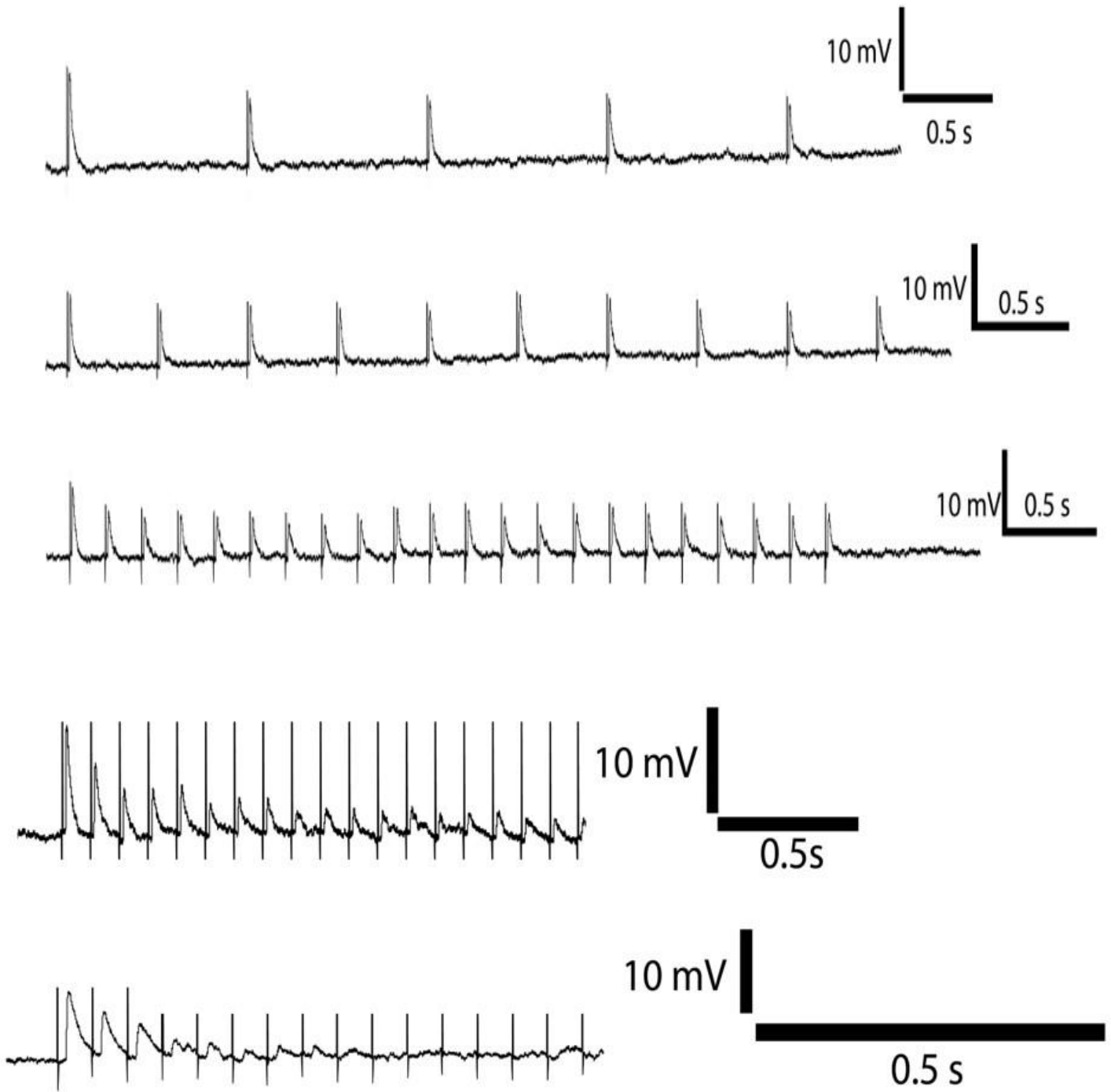

Trace 4.4 Example traces of pelvic-nerve stimulated EPSPs at $1 \mathrm{~Hz}, 2 \mathrm{~Hz}, 5 \mathrm{~Hz}, 10$ $\mathrm{Hz}$ and $20 \mathrm{~Hz}$ in 3-day spinal cord injury. 

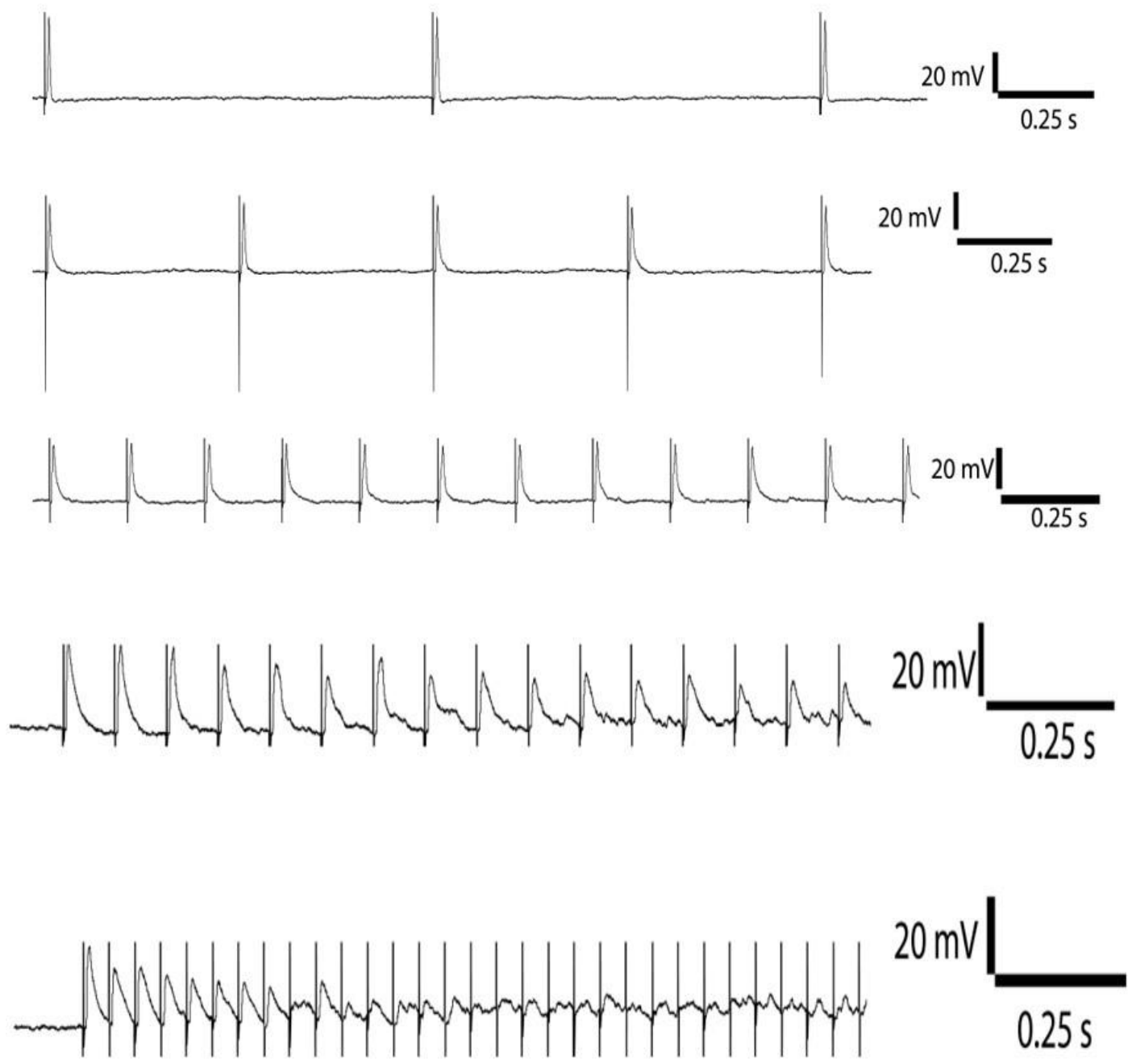

Trace 4.5 Example traces of pelvic-nerve stimulated EPSPs at $1 \mathrm{~Hz}, 2 \mathrm{~Hz}, 5 \mathrm{~Hz}, 10$ $\mathrm{Hz}$ and $20 \mathrm{~Hz}$ in 1-month laminectomy. 

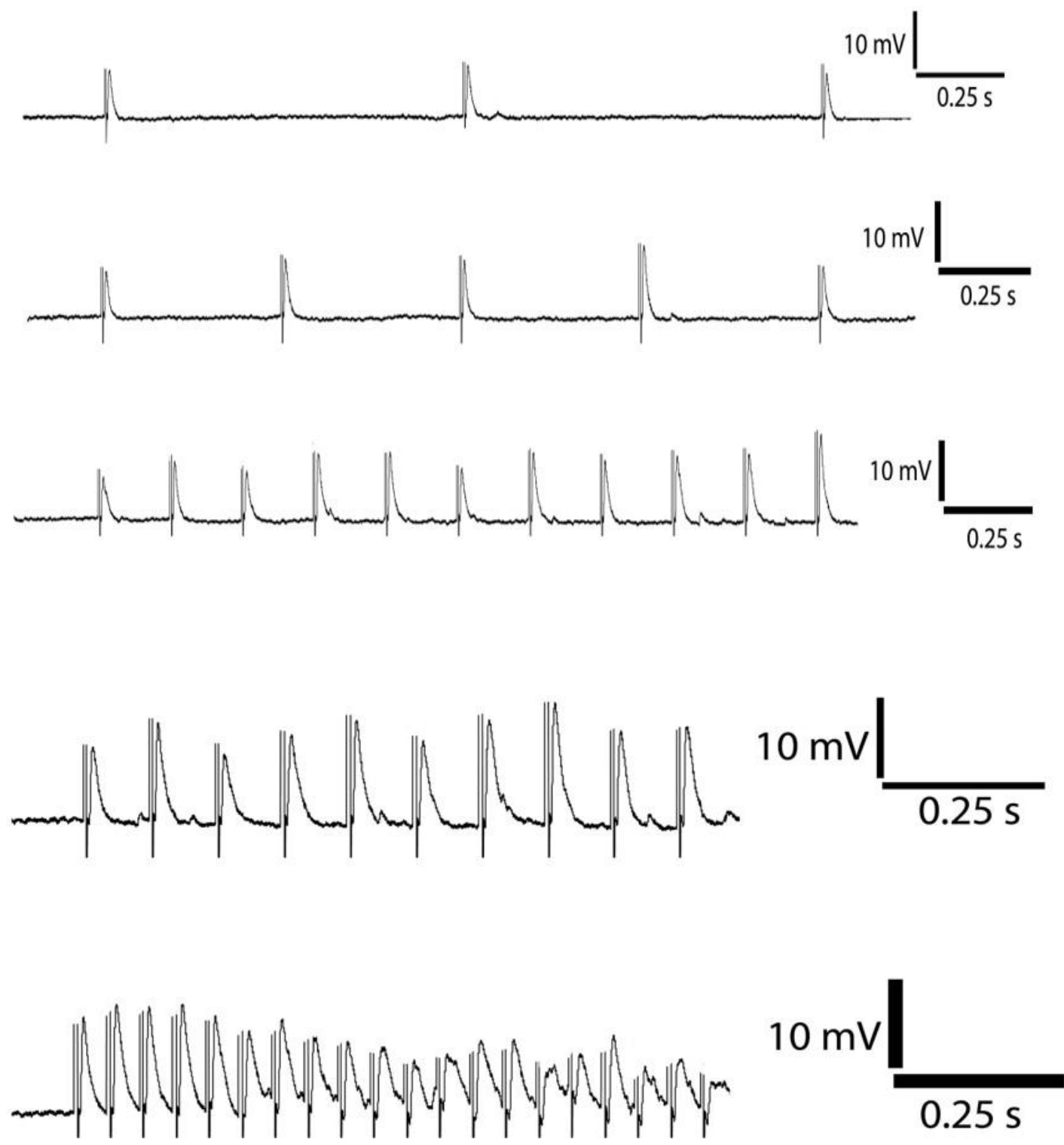

Trace 4.6 Example traces of pelvic-nerve stimulated EPSPs at $1 \mathrm{~Hz}, 2 \mathrm{~Hz}, 5 \mathrm{~Hz}, 10$ $\mathrm{Hz}$ and $20 \mathrm{~Hz}$ in 1-month spinal cord injury. 


\subsubsection{Frequency-dependent modulations of EPSPs}

The pelvic nerve was stimulated at $1 \mathrm{~Hz}, 2 \mathrm{~Hz}, 5 \mathrm{~Hz}, 10 \mathrm{~Hz}$, and $20 \mathrm{~Hz}$ frequencies and the evoked postsynaptic depolarizations were measured in current clamp mode. Trace 4.3 shows representative traces to nerve stimulation at each frequency from the four experimental groups in both males and females. The data from both males and females are presented in combination in all the nerve-stimulation experiments due to lower sample size within individual sexes. The amplitudes of the responses were normalized to the first pulse. The normalized amplitudes from the $1^{\text {st }}$ pulse to the $5^{\text {th }}$ pulse at each frequency are shown as mean $\pm \mathrm{SE}$ for each experimental group. Data were analyzed using Two-way repeated-measure ANOVA for condition (Lam vs. SCI) x pulse (P1 vs. P2.vs.P5).

Within acute injury and laminectomy groups, there were no significant differences between experimental groups nor between pulses within each group at low frequencies (1Hz, and $2 \mathrm{~Hz})($ Fig 4.8). At higher frequencies $(5 \mathrm{~Hz}, 10 \mathrm{~Hz}$, and $20 \mathrm{~Hz})$, differences were found between P1 and P5 in both laminectomy and SCI groups indicating depression of responses (Fig 4.8).

Within chronic injury and laminectomy groups, no significant differences were found between experimental groups nor between pulses within each group at low frequencies $(1 \mathrm{~Hz}$, and $2 \mathrm{~Hz})($ Fig 4.8).

At $5 \mathrm{~Hz}$, differences were found between conditions (1-month laminectomy and 1-month SCI, $\mathrm{P}=0.010$ ) (Fig 4.8). There was also significant interaction between Condition and Pulses. Differences within groups were also found between P1 and P5 in laminectomy 
group $(\mathrm{P}=<0.001)$ indicating depression. Between group differences were found at $\mathrm{P} 5$ $(\mathrm{P}=<0.001)($ Fig 4.8).

At $10 \mathrm{~Hz}$, significant differences between 1-month laminectomy and 1-month SCI were found at $\mathrm{P} 2(\mathrm{P}=0.007)$ and $\mathrm{P} 5(\mathrm{P}=0.014)$. Within group differences were found between $\mathrm{P} 1$ and $\mathrm{P} 5(\mathrm{P}=<0.001$ for lam, $\mathrm{P}=0.014$ for $\mathrm{SCI})$, and $\mathrm{P} 2$ and $\mathrm{P} 5(\mathrm{P}=0.021$ for lam, $\mathrm{P}=0.023$ for $\mathrm{SCI}$ ) in both laminectomy and SCI groups (Fig 4.8).

At $20 \mathrm{~Hz}$, significant differences between 1-month laminectomy and 1-month SCI were found at $\mathrm{P} 2(\mathrm{P}=0.020)$ and $\mathrm{P} 5(\mathrm{P}=0.003)$. Within group differences were found between $\mathrm{P} 1$ and $\mathrm{P} 5(\mathrm{P}=<0.001$ for lam, $\mathrm{P}=0.003$ for $\mathrm{SCI})$, and between $\mathrm{P} 2$ and $\mathrm{P} 5(\mathrm{P}=0.004)$ in laminectomy group (Fig 4.8).

\subsubsection{Frequency-dependent modulation of postsynaptic currents}

The pelvic nerve was stimulated at $1 \mathrm{~Hz}, 2 \mathrm{~Hz}, 5 \mathrm{~Hz}, 10 \mathrm{~Hz}$, and $20 \mathrm{~Hz}$ frequencies while the post-synaptic cells were clamped at $-50 \mathrm{mV}$, and current amplitudes were measured. The results reflected the trends seen with EPSPs in all experimental groups (See Fig 4.8). However, we cannot analyze this set of data accurately because of very low sample sizes, and hence this data set is omitted from reporting. 


\subsection{Reliability of evoked action potentials at multiple frequencies}

3-day Lam AP percentages

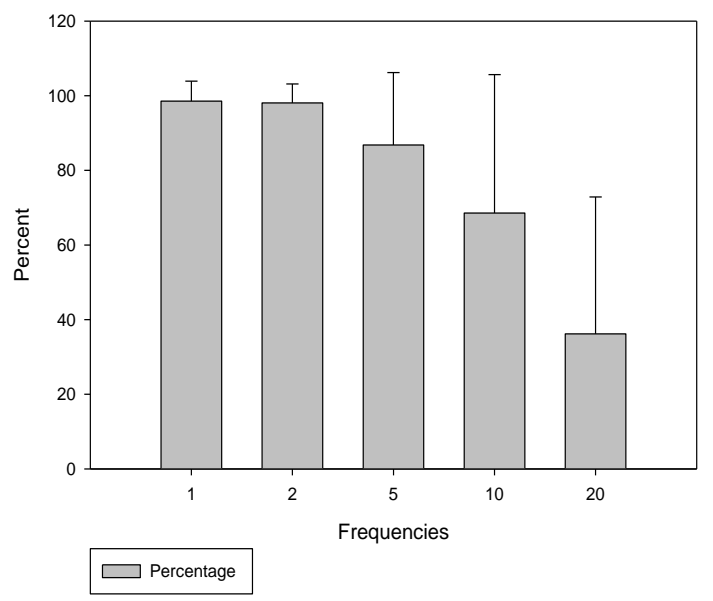

1-month Lam AP percentages

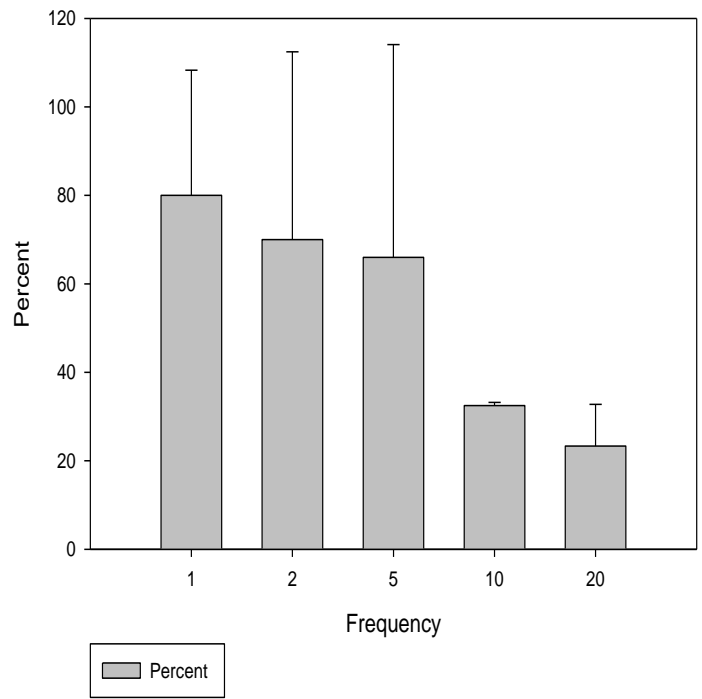

3-day SCI AP percentages

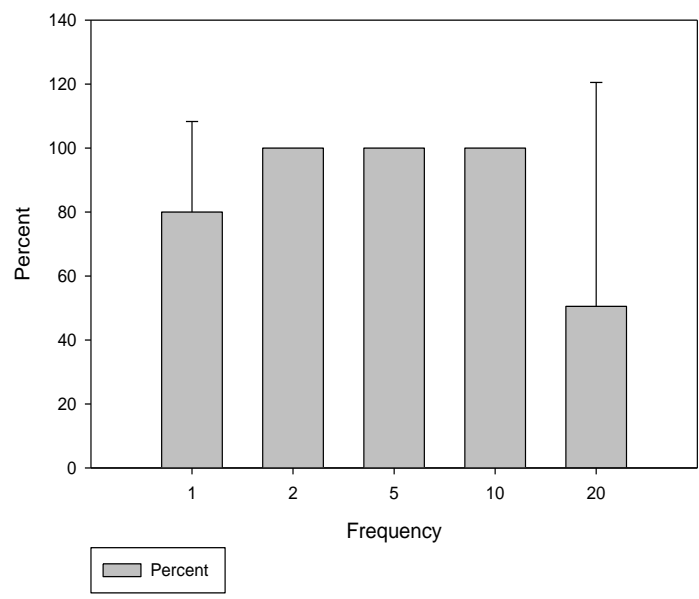

1-month SCI AP percentages

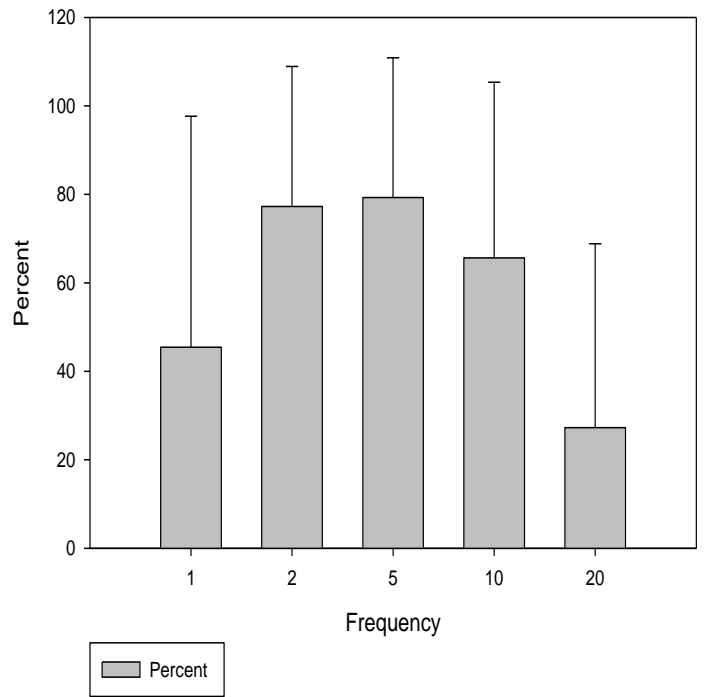

Figure 4.9 Bar graphs showing percentage of action potentials fired in response to pelvic nerve stimulation at different frequencies.

3-day lam n=14, 3-day SCI n=2, 1-month lam n=2, and 28-day (1-month) SCI n=11.

Action potentials from male and female MPGs were recorded in response to pelvic nerve stimulation at $1 \mathrm{~Hz}, 2 \mathrm{~Hz}, 5 \mathrm{~Hz}, 10 \mathrm{~Hz}$, and $20 \mathrm{~Hz}$. The number of action potentials fired 
within the first 5 seconds of each train of stimulation. The number of action potentials evoked are presented as averaged mean + SE percentages for each group at each frequency. Data comparing the percentages between frequencies for each group were analyzed using One-way repeated measures ANOVA test or Friedman Repeated Measure Analysis of Variance on Rank as appropriate.

In 3-day laminectomy group, stimulating the nerve at $1 \mathrm{~Hz}$ evoked the most reliable number of action potentials with the mean of $98.57 \%$ followed by that of $2 \mathrm{~Hz}$ with the mean of $98.07 \%$ (Fig 4.9). The reliability of evoking action potentials significantly declined at $10 \mathrm{~Hz}$ with the mean of $68.5 \%(\mathrm{P}=<0.001)$ and $20 \mathrm{~Hz}$ with the mean of $36.19 \%(\mathrm{P}=<0.001)$ compared to the percentage at $1 \mathrm{~Hz}$ (Fig 4.9). In 3-day SCI group, the action potentials were evoked with the mean of $100 \%$ reliability at $2 \mathrm{~Hz}, 5 \mathrm{~Hz}$, and 10 $\mathrm{Hz}$ (Fig. 9). At $1 \mathrm{~Hz}, 80 \%$ of the pulses triggered action potentials, however, at $20 \mathrm{~Hz}$, action potentials were triggered only $50.5 \%$ of the time (Fig 4.9 ). Mean percentages of action potential responses between 3-day laminectomy and SCI groups at a given frequency were analyzed by using One-Way ANOVA or KruskalWallis One Way Analysis of Variance on Ranks as appropriate. No significant differences were found between the mean percentages at any frequency. The sample size for 3-day SCI was only 2, and hence the negative result should be interpreted with caution.

Significant reductions in percent of action potentials started at $10 \mathrm{~Hz}$ with more reductions at $20 \mathrm{~Hz}$ for the laminectomy groups. Action potential reductions in acute laminectomy was seen only at $20 \mathrm{~Hz}$ to $50 \%$ with maximum percentages from $2 \mathrm{~Hz}$ to 10 $\mathrm{Hz}$ (Fig 4.9). In 1-month laminectomy group, the maximum number of action potentials 
were triggered at $1 \mathrm{~Hz}$ with mean percentage of $80 \%$. The percentages declined with increasing frequencies of stimulation; $70 \%$ at $2 \mathrm{~Hz}, 66 \%$ at $5 \mathrm{~Hz}, 32.5 \%$ at $10 \mathrm{~Hz}$, and $23.335 \%$ at $20 \mathrm{~Hz}$ (Fig 4.9). The action potential firing percentages seem to be at the highest at $2 \mathrm{~Hz}$ and $5 \mathrm{~Hz}$ for chronic SCI with reductions at $10 \mathrm{~Hz}$ and much greater reductions at $20 \mathrm{~Hz}$ (Fig 4.9). In 1-month SCI group, maximum mean percentage of action potentials were triggered at $5 \mathrm{~Hz}(79.27 \%)$ followed by $2 \mathrm{~Hz}(77.27 \%)$ (Fig 4.9). Lower percentages were seen at $1 \mathrm{~Hz}(45.45 \%), 10 \mathrm{~Hz}(65.63 \%)$, and lowest percentage at $20 \mathrm{~Hz}(27.27 \%$ ) (Fig 4.9). At $1 \mathrm{~Hz}$ and $20 \mathrm{~Hz}$, the mean percentages were significantly less when compared to the highest percentage of $79.27 \%$ at $5 \mathrm{~Hz}$ (Fig 4.9). Mean percentages of action potentials between 1-month laminectomy and SCI groups at a given frequency were analyzed using Kruskal-Wallis One Way Analysis of Variance, and no significant differences were found between the two groups at any frequency. The sample size for 1-month laminectomy was only 2 , and hence the negative result should be interpreted with caution.

\subsection{Discussion}

Spinal cord injury (SCI) impairs autonomic nervous system functions including the functions of the lower urinary tract. While there have been studies on the effects of spinal cord injury on afferent division of the reflex pathway as well as the changes in target organ functions, functional study on the efferent neurons after spinal cord injury has not been done. Synaptic transmission at the mouse MPG has been reported to function as a simple relay center with preganglionic inputs generating fast excitatory postsynaptic responses through the actions of nicotinic acetylcholine receptors (nAChRs) (de Groat \& Booth, 1980; de Groat \& Booth, 1993). Recently, studies have suggested roles of MPG as 
a peripheral sensory-motor modulation center of bladder activity (Persyn et al., 2016).

However, we do not know how this synaptic transmission is affected in the case of disease or injury. In this study, we investigated the effects of spinal cord injury on the synaptic transmission, passive and action potential properties of MPG neurons in both acute and chronic stages. These results in our study showed that synaptic and intrinsic properties of major pelvic ganglia (MPG) neurons were altered by spinal cord injury. Changes in these properties were seen throughout the progression of post-injury period. Slight changes in the frequency-dependent responses to preganglionic nerve stimulation were also observed in MPG neurons in chronic spinal cord injured mice.

\subsubsection{Cholinergic transmission at MPG neurons in acute and chronic SCI}

Synaptic properties in terms of acetylcholine (ACh)- activated currents are affected in both male and female mice in spinal cord injured groups. Reductions in ACh-activated current amplitudes suggest reduction in synaptic strength at acute (3-day) SCI. Both males and females in acute SCI groups showed decreased current amplitudes compared to corresponding laminectomy groups although the differences in females were not statistically significant. Hence, our results suggest that synaptic properties at the MPG of males and females are affected by spinal cord injury to various extents in such a way that those of the females seem to be less affected.

The reduction in synaptic strength is also reflected in reduction in mRNA transcript levels for $\alpha 3$ nicotinic acetylcholine receptors (nAChR) subunit, which is one of the major nAChR subunits in major pelvic ganglia (Skok, 2002; De Biasi 2002; Park et al. 2006; Xu et al., 1999; Kyi, unpublished). These results suggest that that reduction in synaptic currents is correlated with downregulation of postsynaptic nicotinic receptor 
mRNA transcripts. Decline in synaptic transmission in association with corresponding decrease in $\alpha 3$ mRNA expressions were also observed in peripheral ganglia in axotomy studies (Yeh et al., 2001; Girard et al., 2013).

The reduction in synaptic strength 3-day post-injury period could be a result of sudden loss of presynaptic activity to the autonomic preganglionic neurons below the injury. As a result of loss of supraspinal presynaptic inputs, spinal neurons below the site of injury go into 'spinal shock' period when there is a sudden shock to the autonomic system below the injury (Ditunno et al., 2004). According to morphological studies done in spinal cord injured animals, alterations in synaptic morphology were seen in spinal circuits below the injury. After spinal cord injury, preganglionic neurons in the sacral parasympathetic nucleus showed synaptic degeneration and decreased number of terminals due to loss of supraspinal inputs while there seemed to be rapid replacements of synapses to the motoneurons in Onuf's nucleus (Beattie at al.,1993). Within 3-day post SCI period, the dendrites of sympathetic preganglionic neurons below the injury were also reported to have retracted (Llewellyn-Smith \& Weaver., 2001; Llewellyn-Smith et al., 2006). Reduced level of synaptic activity in the spinal autonomic neurons could, in turn, result in reduced levels of inputs to the postganglionic neurons at the MPG. We reason that in response to decreased levels of preganglionic synaptic inputs, efferent neurons in MPG respond with decreased synaptic strength and down-regulation of $\alpha 3$ receptor mRNA levels during acute injury period.

Both the synaptic strength and the mRNA expressions of $\alpha 3$ receptors recovered at chronic (28-day) SCI. Interestingly, at chronic SCI, ChAT (Choline acetyltransferase) 
enzyme and $\beta 4$ subunit were downregulated compared to chronic laminectomy group. However, the effects of the downregulation of ChAT and $\beta 4$ on synaptic function seem to be minimum since we did not observe decrease in synaptic currents at chronic SCI. This suggests that regulation of nAChR subunits were taking place at both transcriptional and post-transcriptional levels. Differential regulations of $\alpha 3$ and $\beta 4$ subunits by various neurotrophic and transcription factors could explain the differences in effects of SCI on the nAChR subunits (Zhou et al.,1998; Yeh et al., 2001; Albuquerque et al., 2009; Milton et al., 1996).

Recovery of ACh-induced synaptic currents as well as the mRNA levels of $\alpha 3 \mathrm{nAChR}$ at chronic SCI suggest that there is time-dependent progression in regulation of postsynaptic receptor density at the transcriptional level as well as at the functional level in MPG neurons in chronic SCI. This could be due to the return of activity at the spinal preganglionic autonomic neurons over time as presence of new inputs or synapses have been reported in chronic spinalized animals. The sizes of sympathetic preganglionic neurons and their dendrites seemed to have recovered at 14 days post SCI (LlewellynSmith \& Weaver., 2001;Llewellyn-Smith et al., 2006). The dendritic trees of spinal sympathetic preganglionic neurons were found to have recovered from atrophic state 2weeks after injury in spinal rats (Krenz \& Weaver, 1998). The authors suggested that these new inputs from interneurons contribute to the symptoms of autonomic dysreflexia. It is also reported that hyper-reflexic detrusor activity appears 2 to 12 weeks after spinal injury after spinal shock period during which the bladder is areflexic or flaccid (Yoshimura, 1997). These involuntary reflex contractions in response to sensory stimuli 
cause detrusor-sphinter dysynergia since the sphincter activity persists or recovers in chronic injury period (Yoshimura, 1997; Beattie at al.,1993).

Our results suggest that the mRNA levels of major ganglionic nAChR subunit $\alpha 3$ in correlation with the synaptic strength were regulated based on the altered activity levels of spinal preganglionic inputs as time after injury progresses. Presynaptic input and input-derived signals are known to influence the $\mathrm{nAChR}$ receptor expression in autonomic ganglia during development (Jacob, 1991; Levey et al., 1995; Wang et al., 2002). The expression levels of specific subtypes of neuronal nAChRs are dependent upon both the connections of ganglionic neurons with presynaptic input as well as ganglionic innervation to target organ especially during development (Wang et al., 2002). Our study shows that disruptions in presynaptic signaling pathway such as injury or loss of input in mature peripheral nervous system can also have downstream effects on neurons of peripheral ganglia. Knockout studies in mutant mice that lack $\alpha 3$ and $\beta 4$ receptors were shown to have enlarged bladders and impaired bladder functions (Wang et al., 2002; Xu et al., 1999; Xu et al., 1999(b)). Hence, alterations in the expression of these major ganglionic subunits can, in turn, have functional consequences to the target organs that these MPG neurons innervate.

Our previous study on unilateral decentralization of the MPG showed denervation supersentivity of MPG neurons. In response to loss of preganglionic input, up-regulation of $\alpha 3$ and $\beta 4$ mRNA subunits during both acute (24-hr) and chronic (28-day) post injury were observed. The current responses to acetylcholine was also shown to be increased in decentralized MPGs in correlation with the $\alpha 3$ and $\beta 4$ subunit mRNA expressions at both 
time points (Kyi, to be published). This denervation study and our current study showed that loss or alteration of inputs to MPG neurons in adult mice result in changes in synaptic signaling in the autonomic ganglia.

\subsubsection{Excitability changes of MPG neurons in acute and chronic SCI}

Physiological studies on the efferent neurons of the MPG after SCI have not been carried out while numerous studies have been done on the plasticity of the afferent division of lower urinary tract reflex pathway in SCI. Changes in excitability of ganglionic neurons in response to spinal cord injury have been reported in sensory neurons of the dorsal root ganglia (DRG) (de Groat et al., 1998; Yoshimura, 1997; de Groat et al., 1990; Yoshimura \& de Groat, 1996; de Groat \& Yoshimura, 2006; de Groat \& Yoshimura, 2010). Changes in electrophysiolgical properties of bladder afferent neurons in the DRG have been reported in chronic SCI animals. Takahashi et al, reported alterations in the phenotypes of DRG neurons from phasic to tonic firing patterns in association with downregulation of A-type potassium $\left(\mathrm{K}^{+}\right)$current (Takahashi et al., 2013). The reduction in potassium current was also associated with downregulations protein and mRNA levels of Kv $1.4 \alpha$ subunit (Takahashi et al., 2013). Increased excitability of DRG neurons of chronic SCI animals were also found to have phenotypical changes of sodium $\left(\mathrm{Na}^{+}\right)$channels (Yoshimura \& de Groat, 1996). The phenotypes of the $\mathrm{Na}^{+}$channels changed from highthreshold, tetrodotoxin (TTX)-resistant channels to low-threshold, TTX-sensitive channels (Yoshimura \& de Groat, 1996). The authors attributed the cause of these changes to the neurotrophic signals from the hypertrophied bladder (Yoshimura \& de Groat, 1996; de Groat \& Yoshimura, 2010; 2012). The shapes of the action potentials 
were also reported to have changed into narrower spikes in DRG neurons of the SCI animals (Yoshimura \& Groat, 1997).

We investigated the excitability of neurons by recording the number of action potentials triggered at a given amplitude of intracellular current injection (firing pattern) as well as by measuring the amount of current it takes to fire and action potential (Rheobase). Our results revealed larger number of neurons responded with adapting multiple spikes or tonic spiking patterns in chronic SCI group compared to acute SCI and both laminectomy groups. While most of the neurons responded with phasic one spikers to current injections, more neurons in chronic SCI group were found to respond with intermediate (adapting multiple-spikers) or tonic firing patterns. The threshold to fire action potentials were not different among groups in males. In females, however, the acute SCI group had significantly higher threshold compared to that of acute laminectomy group.

In addition to changes in excitability, we observed downregulation of mRNA transcripts for voltage-gated (L- type) calcium channel (Cacna1d), A-type potassium channel (Kcna4), and persistent sodium channel gene (SCN3A) in chronic SCI group. Alteration in these mRNA levels could contribute to changes in excitability, however, we cannot extract meaningful interpretation from these data because of the very low levels of expression for these genes. These results from our study suggest spinal cord injury causes changes excitability as well as changes in expressions of voltage-gated ion channel expressions in efferent neurons of the MPG. 


\subsubsection{Changes in action potential properties of MPG neurons in acute and chronic SCI}

Different effects of SCI on males and females were continued to be observed in action potential properties from the rebound action potentials. Alterations in action potential properties; antipeak amplitude or afterhyperpolarization (AHP), decay slope and decay time together with changes in shapes of action potentials were seen in males at chronic SCI. Significant reductions in these properties compared to acute SCI as well as chronic laminectomy lead to narrower action potentials with higher amplitudes. Action potential properties of females were affected during acute injury, but they recovered at chronic injury. At acute injury stage action potentials from female SCI groups had smaller peak amplitudes, longer half-width, smaller rise slope, longer rise time were different from those of acute laminectomy group. These properties lead to wider action potentials with shorter amplitudes in acute female SCI groups, however, these action potential properties were back to comparable levels with those of laminectomy groups at chronic SCI stage. It seems that action potential properties of phasic cells have been changed into those of multiple spikers at chronic SCI in males only since those of the females revert back to phasic laminectomy values at chronic stage. These differences in properties of action potentials suggest sex-dependent and also time-dependent effects on spinal cord injury on MPG neurons. No significant changes in the mRNA levels of voltage-gated ion channels were observed at 3-day post SCI suggesting that changes in channel properties do not take place during acute SCI period.

The dichotomy between males and females were also observed in synaptic, intrinsic, and action potential properties among SCI groups. The synaptic and intrinsic properties of the females did not significantly differ between laminectomy and injury groups while those 
of males did. The action potential properties of males were affected at chronic SCI whereas those of the females were affected at acute SCI period. These differences could be due to understandable differences in the target organs that the MPG neurons innervate, and also due to influence of different sex hormones. In our study, the MPG neurons examined were randomly impaled, and could potentially include a mix of neurons that innervate various target organs such as the bladder, the penis, a little bit to prostate, or the distal colon (Wanigasekara et al., 2003) in males, and the bladder, the linings of the vagina and distal colon in females (Jobling \& Lim, 2008). The difference in the nature of target organs could explain the variable effects of SCI on MPG neurons of males and females.

In addition to differences in reproductive organs, sexual dimorphism in bladder function in terms of external urethral sphincter activity of male and female rats has been reported (Chen et al., 2012). The reported differences were seen in 'average volume threshold, amplitudes of bladder contraction, duration and area of contraction, voided urine volume, and inter-contraction interval' (Chen et al., 2012). Gender differences are also reported to affect the recovery of spinal cord injury differently in males and females. Chan et al.,2013, in a review from experimental studies and clinical outcomes, suggest that females seem to have benefits from the gonadal hormones during the recovery. These findings are consistent with our findings in that various cellular properties of MPG neurons in females seem to be more protected from the effects of SCI compared to males. The authors reported that estrogen and progesterone may have neuroprotective effects on females during recovery whereas testosterone may have adverse effects on males recovering from spinal cord injury (Chen et al.,2013). 


\subsubsection{Nerve stimulation experiments on the nature of preganglionic inputs to MPG after $\underline{\mathrm{SCI}}$}

Spinal cord injury is an upstream insult to the MPG neurons since the injury is not a direct decentralization but rather loss of input to the preganglionic neurons in the spinal cord that innervate the MPG neurons. Hence, we asked if the type of input signal from these preganglionic neurons have changed as a result of SCI in both acute and chronic stages. We performed experiments to answer these questions by stimulating the pelvic nerve at different frequencies and recording from the MPG neurons in both current-clamp and voltage-clamp modes.

Although no significant depression of postsynaptic responses between groups was observed at lower frequencies, there seems to be a general trend of depression of voltage responses at higher frequencies. Interestingly, chronic SCI group seems to be depressing the least among all four groups while the acute SCI group seems to be depressing the most. Even at higher frequencies, depression of responses was not observed until later pulses in chronic SCI group.

We also measured the frequency dependence of synaptic responses by measuring EPSCs in voltage clamp mode in order to limit potential contribution of voltage-gated ion channels. While both laminectomy groups seemed to be depressing at higher frequencies, unusual pattern of responses were seen in acute SCI and chronic SCI groups. Chronic SCI group did not show depression at any frequency but instead showed facilitations from $2 \mathrm{~Hz}$ to $20 \mathrm{~Hz}$. Acute SCI group showed facilitation at $10 \mathrm{~Hz}$ followed by minor facilitation at $20 \mathrm{~Hz}$. However, these data are not reported due to very small sample sizes. 
The differences in synaptic responses to different presynaptic input frequencies could be due to a variety of presynaptic and postsynaptic mechanisms including availability of readily releasable presynaptic vesicles, probability of neurotransmitter release, and sensitivity of postsynaptic receptors (Chen et al., 1999; Galarreta \& Hestrin, 1998; Kottick \& Del Negro, 2015; Okatan \& Grossberg, 2000; Miles, 1986).We reasoned that as a consequence of alterations in properties of spinal preganglionic neurons after SCI ( Beattie at al.,1993; Llewellyn-Smith \& Weaver., 2001; Krenz and Weaver 1998; Grossman et al. 1999, 2000), the nature of synaptic output from preganglionic neurons which innervated the MPG neurons will also be altered. While we did not perform experiments to answer mechanistic questions, our data suggest that there could be some alterations in the preganglionic inputs to the MPG since slight facilitations were observed at high frequencies in chronic SCI whereas high frequency stimulation of pelvic nerve results in depression of the responses in laminectomy groups.

In addition to evoked EPSPs, the preferred frequency to reliably fire an action potential in response to repetitive stimulation of pelvic nerve at different frequencies was also tested for all experimental groups. In general, action potentials were generated more reliably at low frequencies $(1 \mathrm{~Hz}$ and $2 \mathrm{~Hz}$ ) but the reliability started to decrease at $10 \mathrm{~Hz}$ and with most reductions at $20 \mathrm{~Hz}$ in laminectomy groups. Acute SCI had wider frequency range of reliably generating action potentials from $2 \mathrm{~Hz}$ to $10 \mathrm{~Hz}$ while chronic SCI group had high percentages at $2 \mathrm{~Hz}$ and $5 \mathrm{~Hz}$ with slight reduction at $10 \mathrm{~Hz}$ but significantly reduced at $1 \mathrm{~Hz}$ and $20 \mathrm{~Hz}$. These results seem to suggest that after SCI, MPG neurons have different ranges of input frequencies to reliably fire an action potential. However, 
we could only make cautious interpretations from this data due to very small sample sizes in acute SCI and chronic laminectomy groups.

Presynaptic neuronal activity and timing of the stimuli can modulate the release of neurotransmitters from presynaptic boutons (Dobrunz, 2002; Wang, 2005). The probability of neurotransmitter release can depend on the size and availability of readily releasable pools of neurotransmitter vesicles which can be a rate-limiting step in the transmitter release process, and the rate constant of release (Fortune \& Rose, 2001; Dobrunz, 2002; Neher \& Sakaba, 2008; Fortune \& Rose, 2001; Bruckner et al.,2017). The amount of calcium influx caused by the depolarization of the presynaptic neuron can alter the release probability of the release of vesicles (Dobrunz, 2002; Xu \& Wu, 2005; Neher \& Sakaba, 2008; Alabi \& Tsien, 2012; Bruckner et al., 2016; Dajas-Bailador \& Wonnacott, 2004; Albuquerque et al., 2009). High levels of intracellular calcium levels can increase the number of readily releasable vesicles and involved in 'priming' of the release (Alabi \& Tsien, 2012; Neher \& Sakaba, 2008; Bruckner et al., 2016) and coupling between releasable vesicles and calcium channels possibly through phosphorylation of synaptic proteins (Neher \& Sakaba, 2008). Frequency-dependent depressions could be due to a decrease in release probability of the vesicles or depletion of the releasable vesicles at high-probability synapses whereas facilitation is reported to be due to build-up of residual calcium in the synaptic bouton at low-probability synapses (Abbott \& Regehr, 2004; Dobrunz, 2002; Neher \& Sakaba, 2008; Fortune \& Rose, 2001).

Depolarization of presynaptic membrane is also reported to cause calcium-dependent increase in choline transporter (CHT) activity (Ferguson et al., 2003). In central and 
peripheral cholinergic synapses, $\mathrm{CHT}$ is required to recover ACh hydrolyzed by acetylcholinesterase (AChE) from the extracellular space (Ferguson et al., 2003). Ferguson et al., implied that presynaptic neuronal activity can drive the CHT localized on the presynaptic vesicles to the plasma membrane and regulate the ACh reuptake process to keep up with the release of ACh (Ferguson et al., 2003).

In addition to modulation of neurotransmitter release based on presynaptic activity, synapses have the ability to prefer certain frequency of inputs over others and hence, they can act as 'filters' of information (Abbott \& Regehr, 2004). Synapse that transmit neural signals reliably at low frequencies are called 'low-pass' filters, those that reliably transmit signals at high frequencies are called 'high-pass' filters, and those that prefer intermediate frequencies are called 'band-pass' filters (Abbott \& Regehr, 2004). These synapses can change their phenotypes based on the pattern or nature of inputs they receive (Abbott \& Regehr, 2004).

Changes in the reflex mechanisms controlling lower urinary tract as a result of hyperexcitability of DRG neurons and plasticity of afferent pathways have been reported after spinal cord injury (de Groat et al., 1998; de Groat \& Yoshimura, 2010, 2012; Yoshimura \& de Groat, 1996; de Groat, 1995; Vizzard, 2006). However, we do not know the nature or the pattern of autonomic efferent outputs from spinal autonomic neurons after spinal cord injury. We know that there is an increased afferent activity which drives the hyperreflexic bladder in chronic SCI. A morphological study by Kruse et al., reported hypertrophy of DRG neurons in SCI but the authors did not find significant hypertrophy of MPG neurons (Kruse et al., 1995). 
Our results indicate that at acute SCI, synaptic properties of both males and females are affected. Action potential properties of females in acute injury changes in the way that they result in wider and shorter action potentials. At chronic SCI, changes in action potential properties are seen only in males and those changes overshoot the laminectomy values possibly contributing to hyperexcitability of those neurons. There are more intermediate and tonic spiking neurons at chronic SCI stage compared to acute injury or laminectomy groups. These changes were also reflected at the level of mRNA transcripts for corresponding receptors and ion channels underlying these properties. These results suggest possible activity-dependent reconfiguration of post-synaptic properties as well as action potential properties in response to possibly altered inputs after spinal cord injury. Hence, the data suggests that reconfigurations after loss of physiological input were taking place at the transcriptional level in time-dependent manner in the efferent neurons of the MPG.

Future studies on voltage-gated ionic currents at the MPG neurons before and after SCI will reveal the underlying mechanisms responsible for changes in action potential and firing properties. Molecular studies on regulation of synaptic transmission by presynaptic activity and underlying signaling pathways behind the plasticity of the postsynaptic MPG neurons will give us insights into how the synaptic transmission of the efferent neurons at the autonomic ganglia are affected by SCI. Understanding the impact of spinal cord injury at the major pelvic ganglia will assist in understanding the plasticity of the peripheral ganglionic neurons in response to CNS injury. The results from this study provide us with information on the effects of SCI on peripheral ganglia that eventually 
results in organ or system level functional alteration. Our results also indicate that potential target for therapeutic measures should take sex differences into account. 


\section{CHAPTER 5 SUMMARY AND CONCLUSIONS}

The goal of this thesis was to understand if and how the loss of physiological inputs to the neurons of the major pelvic ganglia (MPG) affect the properties of MPG neurons. Major pelvic ganglia contain postganglionic neurons that innervate the urogenital organs. The functions of these urogenital organs are among the autonomic functions that are compromised after spinal cord injury. Hence, we sought to understand how the injury affects the functions of MPG neurons as these neurons directly innervate the urogenital organs and play a role in regulating proper functioning of the target organs. Specifically, we were interested in understanding the synaptic, passive, and firing properties of the MPG neurons in normal physiological state as well as in injury states. We approached our questions through three main projects as described in the three data chapters of this thesis.

In chapter (2), I characterized the ganglionic cholinergic synaptic transmission, passive properties and firing properties of major pelvic ganglia (MPG) neurons in male and female adult Swiss-Webster mice. Our results indicated that synapses in MPG neurons act as low pass filters since frequency-dependent depression of synaptic responses were observed at higher frequency of pelvic nerve stimulations. In response to nervestimulation, approximately half of the population of the neurons tested responded with all-or-none suprathreshold potentials (high safety factor) while the other half responded with subthreshold potentials (low safety factor) indicating heterogenous nature of synapses within the ganglia. There were no significant differences in passive, action potential properties of MPG neurons between male and female mice. Our pharmacology 
study and analyses of mRNA transcripts for nicotinic acetylcholine receptor (nAChR) subunit genes revealed that, in accordance with other autonomic ganglia, $\alpha 3$ and $\beta 4$ subunits are the major subunits involved in the ganglionic transmission at the MPG.

In chapter (3), I investigated the effects of unilateral decentralization (transection of pelvic nerve and hypogastric nerve) on synaptic, and membrane properties of ipsilateral and contralateral MPG neurons in adult, male Swiss-Webster mice. Our results indicated increased synaptic currents as well as upregulation of $\alpha 3$ and $\beta 4$ subunits in both ipsilateral and contralateral ganglia. There also seemed to be compensation for the loss of preganglionic inputs to the decentralized ganglia from contralateral ganglia presumably due to feedback signals from the bladder. Our results indicate the properties of "denervation supersensitivity", in peripheral ganglia as is the case in denervated muscles. In addition, we also learned the ability of the contralateral ganglia to compensate for the loss of inputs in the ipsilateral decentralized ganglia.

In chapter (4), I studied the effects of complete spinal transection (between T10 and T11 spinal segments) on cholinergic transmission, passive, and firing properties of MPG neurons. W used adult male and female transgenic mice (B6. Cg-Tg) labelled with eGFP for ChaT (choline acetyltransferase enzyme) which labeled parasympathetic neurons in the MPG. Our results suggested that 3-days after spinal cord injury (acute injury period), there was a decline in cholinergic transmission together with downregulation of $\alpha 3$ subunit mRNA transcripts. However, at 28 days after spinal transection, the synaptic transmission recovered as did the $\alpha 3$ subunit mRNA levels. In response to pelvic nerve stimulations at the frequencies of $1 \mathrm{~Hz}, 2 \mathrm{~Hz}, 5 \mathrm{~Hz}, 10 \mathrm{~Hz}$ and $20 \mathrm{~Hz}$, chronic spinal 
transected group showed reduced levels of frequency-dependent depression of synaptic responses (EPSPs) at higher frequencies $(10 \mathrm{~Hz}$ and $20 \mathrm{~Hz})$ as were the cases with the acute spinal transected group and laminectomy groups. Synaptic currents (EPSCs) from the chronic spinal groups also showed least depression when stimulated at high frequencies $(10 \mathrm{~Hz}$ and $20 \mathrm{~Hz})$ when the acute injury and laminectomy groups showed depression of current responses with higher frequencies of stimulations. Hence, our results suggest that MPG synapses can be modulated to match the input frequency after SCI, assuming higher frequency of preganglionic inputs originating from preganglionic neurons on the spinal cord due to hyperactive sensory inputs and hyperreflexic bladder functions in chronic SCI. We also observed hypertrophy of bladders in chronic SCI animals. It is possible that presynaptic plasticity involved with alterations form frequency-dependent depression to facilitation in chronic SCI. Our observation of recovery of $\alpha 3$ receptor mRNA levels suggest that postsynaptic receptor density was also recovering over time after injury. Hence, MPG synapses are dynamic and plastic depending on the frequency of inputs. We observed sex differences in the synaptic and action potential properties of MPG neurons from spinal cord injured mice which we did not observe in laminectomy groups. Female MPG neurons seemed to be less affected by the injury than males' and hence sexual dimorphism should be taken into account in finding out with therapeutic measures and agents for the urogenital dysfunctions after spinal cord injury. Hormonal regulation of MPG neurons after injury is one of the areas that should be studied further. Excitability of the MPG neurons also changed in response to SCI with changes in action potential properties. Hence, the plasticity of underlying ionic mechanisms is another important area of future studies. 
These plastic changes in synaptic, firing and action potential properties of MPG neurons could be due to influences by changes in presynaptic inputs or by retrograde trophic factors and signaling molecules from target organs due to reasons based on evidences discussed below.

We found increased synaptic responses to acetylcholine as well as upregulation of $\alpha 3$ and $\beta 4$ nicotinic acetylcholine receptor subunit genes in decentralized MPGs as well as in contralateral MPGs in chapter (3). In these experiments, the decentralized ganglia did not have any synaptic inputs, however, their axons that innervate the target organs were still intact. With the decentralization of inputs, sensory axons from the target organs to the spinal cord would also be severed, while some sensory feedback signals from the target organs to the ganglia were still intact. Loss of presynaptic inputs while the connections with the target organs are intact triggered supersensitivity of the nAChRs in ipsilateral decentralized ganglia. This could be due to the feedback signals from the target organ to the MPG neurons indicating lack of or reduced activity from the MPG neurons after removal of inputs or the response of MPG neurons to abrupt loss of presynaptic inputs (Tuttle et al., 1994).

Widely reported signals of such kind include nerve growth factors (NGF), and Neuregulin-1 secreted from the bladder or endogenously from the decentralized MPG neurons themselves (Vizzard, 2000, 2006; Kim et al., 2015, 2013; Yeh et al., 2001; Tuttle et al., 1994). These neurotrophic factors are also reported to regulate the expression of nicotinic acetylcholine receptor (nAChR) expressions in adult autonomic neurons (Kim et al.,2015). Reduced levels of acetylcholine esterase enzyme have also been reported as 
one of the mechanisms for denervation-induced supersensitivity of nAChRs (Klingman 1969; Klingman \& Klingman, 1968). Growth and differentiation factors Neuregulin 1(NRG1) possibly secreted by the MPG neurons themselves or retrogradely from the bladder in response to loss of preganglionic inputs can also regulate the levels of acetylcholine esterase activity (Kim et al., 2015; Dougherty et al., 2013).

We did not observe signs of impaired bladder function possibly because the contralateral ganglia were still intact in our unilaterally decentralized animals and seemed to be compensating for reduced outputs from the decentralized ganglia. Firing of postganglionic nerves on the denervated side the bladder in response to stimulation of intact pelvic nerve on the contralateral side has been reported in rats with unilateral MPG decentralization (de Groat \& Kawatini, 1989). The authors reported that this pattern of cross-excitation had not been observed in normal animals indicating the emergence of inter ganglionic pathways because of loss of preganglionic input to MPG on one side.

Persyn et al. also reported that decentralization of rat MPGs did not alter the bladder function whereas removal of MPGs increased non-voiding contractions of the bladder implying intraganglionic peripheral modulatory role of the MPG (Persyn et al., 2016). Hence, the feedback connection with target organ could be one of the factors influencing the functions of the MPG. This feedback from the target organs could be regulating the mRNA synthesis of nAChRs in the MPG neurons.

In axotomy studies, initial decline of nicotinic acetylcholine transmission with downregulation of $\mathrm{nAChR}$ genes in acute state has been reported, however, the receptor levels and the synaptic transmission recovered over time. In axotomy, the presynaptic 
inputs to the ganglia were still intact, however, the axonal contacts with the target organs were lost. Afferent outputs from target organs will also be cut as a result of severing of axonal contacts. The absence of peripheral influence could be a possible factor in downregulation of synaptic transmission in acutely axotomized MPG. Loss of retrogradely transported target-derived nerve growth factors (NGF) have also been reported as an explanation for decline in peripheral synaptic transmission and $\mathrm{nAChR}$ levels at the axotomized superior cervical ganglia neurons of rats and guinea-pigs (Zhou et al., 1998; Purves, 1975).

In acute spinal cord injury, the presynaptic inputs and the axonal connections with the target organs are still intact. However, during the initial period during the spinal shock phase, the bladder is areflexic and hence the preganglionic inputs originating from the spinal cord might be significantly reduced. We observed decreased cholinergic synaptic transmission together with downregulation of $\alpha 3$ receptors during this period. This could be due to loss of either the target organ activity or preganglionic activity originating from the spinal cord. Interestingly, this apparent reduction in preganglionic activity during the acute injury period did not trigger upregulation of synaptic transmission as we observed with decentralization. This could be because of lack of retrograde signals from the target organs that can regulate the expression of nAChRs and acetylcholine esterase enzymes during this spinal shock period. It is also possible that minimal level of afferent activity was still preserved during this stage and hence there is ongoing maintenance of preganglionic input activity to the MPG neurons. Hence, unlike complete loss of synaptic inputs as in the case with decentralization, some level of presynaptic activity could be 
maintained at the MPG. Hence, this did not trigger the mechanisms for denervation supersensitivity of MPG neurons in acute spinal cord injury.

In chronic spinal cord injury, both the activity of the target organ and the presynaptic inputs are recovered due to recovery of spinal reflex mechanisms. We observed recovery of synaptic transmission as well as that of $\alpha 3$ receptors in the MPG of chronic spinal animals at 28-day post-surgery. However, these reflexes appeared in hyperactive form with hyperactive and hypertrophied bladders as observed in our study and as reported in literature (Yoshimura \& de Groat, 1996). Preganglionic axons originating from spinal preganglionic neurons could be experiencing alterations after spinal cord injury due to increased afferent sensory inputs (Yoshimura \& de Groat, 1996). Hence, it is possible that the neurotransmitter release properties from these preganglionic axons are also be altered after spinal cord injury (discussed in detail in chapter 3 ). We cannot rule out the influence of presynaptic activity on the MPG neurons since our results showed reductions in frequency-dependent depression of synaptic responses in chronic SCI suggesting changes in transmitter release properties of preganglionic parasympathetic axons (Chapter 4).

We observed that the firing patterns of some MPG neurons have changed from singlespiking (phasic or fast-adapting) to multiple-spiking (slow-adapting and non-adapting or tonic) phenotypes. We also observed changes in action potential properties to shorter action potentials with higher peak amplitudes in chronic stage. These alterations of postganglionic neurons in firing and action potential properties of MPG neurons could be due to the need to keep up with the higher activity of preganglionic inputs. 
Target organ activity has also been reported to regulate the ganglionic nAChR levels in neurons of the rat pelvic ganglia in rats with urethral-outlet-obstruction (Chung et al., 2015). Chung et al. reported upregulation of acetylcholine-evoked currents together with the mRNA transcripts for $\alpha 3$ and $\beta 4$ subunits in parasympathetic bladder neurons in response to urethral outlet obstruction (Chung et al.,2015). Decline in synaptic transmission in autonomic ganglia due to loss of axonal connections with the target organs have been reported in several axotomy studies. Brenner \& Johnson reported rapid decline in ganglionic transmission in chick ciliary ganglion 3-4 days after axotomy (Brenner \& Johnson, 1976). Girard et al. reported that in response to cavernous nerve injury, the acetylcholine receptor mRNA subunits $\alpha 3, \beta 4$, and $\alpha 7$ were significantly depressed in correlation with decrease in ACh-induced current levels 3days after injury in male mouse MPG (Girard et al., 2013). As found in our study, decreased expression of neuronal nicotinic $\mathrm{ACh}$ receptors (nAChRs) at synapses on postganglionic neurons is one of the mechanisms thought to underlie this synaptic depression (Purves, 1975). Nerve growth factors from the target organs have been reported to be signals involved in regulation of synaptic transmission in the peripheral ganglia (Vizzard, 2000, 2006). Nerve growth factor (NGF) protein levels were reported to have downregulated in acute spinal cord injury but upregulated in the bladders of chronic spinal cord injured rats (Vizzard, 2000, 2006). Hence, these evidences suggest that target-derived influences might be one of the factors regulating the cholinergic neurotransmission of MPG neurons in both acute and chronic spinal cord injury.

The alterations of MPG neuron properties in chronic SCI were only observed in males (Chapter 4). Hence, there could be hormonal influences on the regulation of MPG 
properties. Thus, sex differences in regulation of MPG neuronal functions in physiology and injury states should be taken into account (Kanjhan et al., 2003; Chan et al., 2013).

Within the scope of our experiment, we cannot differentiate with certainty the influence of target organ function from the influence of alteration of presynaptic inputs. In order to clarify these questions, future studies on MPG neurons could be performed on axotomy of MPG together with spinal cord injury in order to eliminate the influence from the target organs. In addition, decentralization studies in spinal cord injured mice could also be performed in order to eliminate the influence of presynaptic inputs and examine if the influence from the target organs alone could alter the properties of MPG neurons in spinal cord injury. Future experiments can also specifically focus on the groups of neurons that innervate specific target organs such as bladder, reproductive organs or the distal colon using retrograde-labeling techniques to study if there is any organ-specific effects of spinal cord injury on the MPG.

Current measures for bladder management in spinal cord injured patients include anticholinergics to reduce the sensation of bladder filling, antimuscarinics, and botulinum toxin to reduce the contraction of bladder detrusor muscle (Eastham \& Gillespie 2013; Apostolidis, 2011). These agents are usually taken in conjunction with catherization of the bladder. However, non-specificity of these agents and side effects make them less desirable. Frequent catheterization also makes the patients susceptible to urinary tract infections (Chew et al., 2013). Various modes of neuromodulation-based prosthetics such as sacral anterior root stimulator, and pudendal modulations have been developed in animal models of bladder dysfunction after spinal cord injury (Chew et al., 2013). 
However, these procedures involve invasive and non-reversible procedures such as rhizotomy or transection of dorsal nerve roots which can affect the nerves innervating other peripheral structures such as the reproductive organs, and removal of external urethral sphincter.

Identifying specific targets is important in order to develop more efficient therapeutic agents with fewer generalized side effects in treating disorders. General anticholinergics used in bladder overactivity require doses higher than recommended dose with significant side effects (Cruz \& Cruz, 2011). Due to the widespread functioning of the cholinergic system in the body, it is important to uncover the receptor subtypes affected in a given pathological condition (Albuquerque et al., 2009). The phenotypic assembly of the subunits also varies with specific target organ (Skok, 2002). Therefore, it is important to selectively study the expression patterns of each subtype of nAChRs and their synaptic properties at MPG neurons that innervate specific urogenital organs in both control and injury states.

Understanding the role of parasympathetic and sympathetic neurons in the major pelvic ganglia and their altered function states after peripheral nerve injury and spinal cord injury will be valuable to discover more specific and target organ-oriented interventions at the level of major pelvic ganglia (Eg. Targeted gene-delivery based therapies). The results from our study have brought us one step closer to understanding how the neurons of the major pelvic ganglia that control the urogenital organs are affected by spinal cord injury and possible mechanisms causing the alterations in the neural functions at the ganglionic level. We hope that the findings from our study would contribute to the future 
research endeavors to effectively control the urogenital functions in spinal cord injured patients. 


\section{BIBLIOGRAPHY}

Abbott, L. F., and Wade G. Regehr. 2004. "Synaptic Computation.” Nature 431 (7010): 796-803. doi:10.1038/nature03010.

Alabi, AbdulRasheed A, and Richard W Tsien. 2012. "Synaptic Vesicle Pools and Dynamic." Old Spring Harbor Perspectives in Biology 4 (8): 1-19.

doi:10.1101/cshperspect.a013680.

Albuquerque, E X, E F Pereira, M Alkondon, and S W Rogers. 2009. "Mammalian Nicotinic Acetylcholine Receptors: From Structure to Function." Physiol Rev 89 (1): 73-120. doi:10.1152/physrev.00015.2008.

Aldahmash, A., \& Atteya, M.2011. "Ganglionectomy in the adult male rat increases neuronal size and synaptic density in remaining contralateral major pelvic ganglion." Current Neurobiology. 2(1) 5-15.

Apostolidis, A. 2011. "Antimuscarinic Treatment for Overactive Bladder Symptoms: An All-Time Classic, yet Still a Clinical and Basic Challenge.” Eur Urol 59 (3): 35658. doi:10.1016/j.eururo.2010.12.022.

Axelsson, B Y J, and S Thesleff. 1959. " A Study of Supersensitivity in denervated mammalian skeletal muscle." J. Physiol. (1957) I49: 178-I93

Bankenahally \& Krovvidi. 2016. "Autonomic nervous system: anatomy, physiology, and relevance in anaesthesia and critical care medicine." BJA Education, 16 (11): 381387 (2016) doi:10.1093/bjaed/mkw011)

Barbeau H, Bedard P. 1981."Denervation supersensitivity to 5-hydroxytryptophan in rats following spinal transection and 5,7-dihydroxytryptamine injection."

Neuropharmacology. (20):611-616.

Beattie MS, Leedy MG, Bresnahan JC. 1993. "Evidence for alterations of synaptic inputs to sacral spinal reflex circuits after spinal cord transection in the cat." Exp Neurol 123:35-50.

Berkley, K.,Robbins, A.,Sato,Y.(1993). Functional differences between afferent fibers in the hypogastric and pelvic nerves innervating female reproductive organs in the rat. Journal of Neurophysiology. 69 (2), 533-544

Biasi, M De. 2002. "Nicotinic Mechanisms in the Autonomic Control of Organ Systems." J Neurobiol 53 (4): 568-79. doi:10.1002/neu.10145. 
Birder, L, W de Groat, I Mills, J Morrison, K Thor, and M Drake. 2010. "Neural Control of the Lower Urinary Tract: Peripheral and Spinal Mechanisms." Neurourol Urodyn 29 (1): 128-39. doi:10.1002/nau.20837.

Brenner, B Y H R, and E W Johnson. 1976. "Physiological and Morphological Effects of Post-ganglionic Axotomy on Presynaptic nerve Terminals." J. Physiol. 260:143-158.

Brown, D. A. (1969). Responses of normal and denervated cat superior cervical ganglia to some stimulant compounds. The Journal of Physiology, 201(1), 225-236.

Bruckner, Joseph J., 1 Zhan, Hong 2 Gratz, Scott J., 2 Rao, Monica, 1 Ukken, Fiona, 2 Zilberg, Gregory, 2 and, and 3 O'Connor-Giles, Kate M.1, 2. 2016. "Fife Organizes Synaptic Vesicles and Calcium Channels for High-Probablility Neurotransmitter Release.” J. Cell Biol 216 (1): 231-46. doi:10.1083/jcb.201601098.

Cannon, W.B; \& Rosenblueth, A. 1949. "Supersensitivity of denervated structures: a law of denervation"Am.J.Med.Sci (198)

Chan, W; Mohammed. Y;Lee.I; Pearse. D.2013. "Effect of Gender on Recovery after Spinal Cord Injury." Transl. Stroke Res. 4:447-461.

Chen, C Y, J M Horowitz, and a C Bonham. 1999. “A Presynaptic Mechanism Contributes to Depression of Autonomic Signal Transmission in NTS." The American Journal of Physiology 277 (4 Pt 2): H1350-60.

Chen, Shih-Ching, Chien-Hung Lai, Wen-Jia Fan, and Chih-Wei Peng. 2012. "Sex Differences in the External Urethral Sphincter Activity of Rats." Journal of Experimental \& Clinical Medicine 4 (3): 157-64. doi:10.1016/j.jecm.2012.04.006.

Chew, D J, L Zhu, E Delivopoulos, I R Minev, K M Musick, C A Mosse, M Craggs, et al. 2013. "A Microchannel Neuroprosthesis for Bladder Control after Spinal Cord Injury in Rat." Sci Transl Med 5 (210): 210ra155. doi:10.1126/scitranslmed.3007186.

Chung, H C, C K Lee, K H Park, and S W Jeong. 2015. "Bladder Outlet Obstruction Causes up-Regulation of Nicotinic Acetylcholine Receptors in Bladder-Projecting Pelvic Ganglion Neurons.” Brain Res. doi:10.1016/j.brainres.2015.01.026.

Cruz, C D, and F Cruz. 2011. "Spinal Cord Injury and Bladder Dysfunction: New Ideas about an Old Problem." Scientific WorldJournal 11: 214-34. doi:10.1100/tsw.2011.26.

Dail, W G. 1996. "The Pelvic Plexus: Innervation of Pelvic and Extrapelvic Visceral Tissues." Microsc Res Tech 35 (2): 95-106. doi:10.1002/(SICI)10970029(19961001)35:2<95::AID-JEMT1>3.0.CO;2-\#. 
Dajas-Bailador, F, and S Wonnacott. 2004. "Nicotinic Acetylcholine Receptors and the Regulation of Neuronal Signalling." Trends Pharmacol Sci 25 (6): 317-24. doi:10.1016/j.tips.2004.04.006.

De Groat, W., \&Booth, A.M.1980." Inhibition and facilitation in parasympathetic ganglia of the urinary bladder." Fed Proac.39(12), 2990-2996.

De Groat, W. C., Kawatini, M., Hisamitsu, T., Cheng, C.-L., MA, C. P., Thor, K., Steers, W. \& RoPPOLO, J. R. 1990." Mechanisms underlying the recovery of urinary bladder function following spinal cord injury." Journal of the Autonomic Nervous System 30, S71-78.

Delacroix Jr., S E, and J C Winters. 2010. "Voiding Dysfunction after Pelvic Colorectal Surgery." Clin Colon Rectal Surg 23 (2): 119-27. doi:10.1055/s-0030-1254299.

Ditunno, J. F., J. W. Little, A. Tessler, and A. S. Burns. 2004. "Spinal Shock Revisited: A Four-Phase Model.” Spinal Cord 42 (7): 383-95. doi:10.1038/sj.sc.3101603.

Ditunno Jr., J F, and C S Formal. 1994. "Chronic Spinal Cord Injury." N Engl J Med 330 (8): 550-56. doi:10.1056/nejm199402243300808.

Dobrunz, Lynn E. 2002. "Release Probability Is Regulated by the Size of the Readily Releasable Vesicle Pool at Excitatory Synapses in Hippocampus." International Journal of Developmental Neuroscience 20 (3-5): 225-36. doi:10.1016/S07365748(02)00015-1.

Dougherty, Kimberly J., Laskaro Zagoraiou, Daisuke Satoh, Ismini Rozani, Staceyann Doobar, Silvia Arber, Thomas M. Jessell, and Ole Kiehn. 2013. "Locomotor Rhythm Generation Linked to the Output of Spinal Shox2 Excitatory Interneurons." Neuron 80 (4). Elsevier Inc.: 920-33. doi:10.1016/j.neuron.2013.08.015.

Eastham, J E, and J I Gillespie. 2013. "The Concept of Peripheral Modulation of Bladder Sensation.” Organogenesis 9 (3): 224-33. doi:10.4161/org.25895.

Fambrough, D M. 1979. "Control of Acetylcholine Receptors in Skeletal Muscle." Physiological Reviews 59 (1): 165-227. doi:10.1007/BF01674266.

Felix, B., Catalin, D., Miolan, J-P., Niel, J-P. 1998. Integrative properties of the major pelvic ganglion in the rat. Journal of the Autonomic Nervous System, 69, 6-11.

Ferguson, Shawn M, Valentina Savchenko, Subbu Apparsundaram, Melissa Zwick, Jane Wright, Craig J Heilman, Hong Yi, Allan I Levey, and Randy D Blakely. 2003. "Vesicular Localization and Activity-Dependent Trafficking of Presynaptic Choline Transporters." The Journal of Neuroscience : The Official Journal of the Society for Neuroscience 23 (30): 9697-9709. doi:23/30/9697 [pii]. 
Fleming, W.W.,McPhillips, J.J.,Westfall, D.P. 1973. Postjunctional supersensitivity and subsensitivity of excitable tissues to drugs. In: Ergebnisse der Physiologie Reviews of Physiology, Volume 68. Ergebnisse der Physiologie, biologischen Chemie und experimentellen Pharmakologie, vol 68. Springer, Berlin, Heidelberg.

Fortune, Eric S., and Gary J. Rose. 2001. "Short-Term Synaptic Plasticity as a Temporal Filter." Trends in Neurosciences 24 (7): 381-85. doi:10.1016/S0166-2236(00)01835-X.

Fowler, C J, D Griffiths, and W C de Groat. 2008. "The Neural Control of Micturition." Nat Rev Neurosci 9 (6): 453-66. doi:10.1038/nrn2401.

Furber, S., Oppenheim, R. W., and Prevette, D. 1987. "Naturally- occurring neuron death in the ciliary ganglion of the chick embryo following removal of preganglionic input: evidence for the role of affer- ents in ganglion cell survival." J. Neurosci. 7: 1816-1832.

Gabella G, Berggren T, Uvelius B.1992. "Hypertrophy and reversal of hypertrophy in rat pelvic ganglion neurons." J Neurocytol 21:649-662.

Gabella, G., Uvelius, B.1993. "Effect of decentralization or contralateral ganglionectomy on obstruction-induced hypertrophy of rat urinary bladder muscle and pelvic ganglion." J Neurocytol 22:827-834.

Gallagher, J.P., Griffith, W.H.,Shinnick-Gallagher, P. 1982. "Cholinergic transmission in cat parasympathetic ganglia." J Physiol 332: 473-486.

Galarreta, Mario, and Shaul Hestrin. 1998. "Frequency-Dependent Synaptic Depression and the Balance of Excitation and Inhibition in the Neocortex." Nature Neuroscience 1 (7): 587-94. doi:10.1038/2822.

Girard, B M, L A Merriam, J D Tompkins, M A Vizzard, and R L Parsons. 2013. "Decrease in Neuronal Nicotinic Acetylcholine Receptor Subunit and PSD-93 Transcript Levels in the Male Mouse MPG after Cavernous Nerve Injury or Explant Culture." Am J Physiol Renal Physiol 305 (10): F1504-12. doi:10.1152/ajprenal.00343.2013.

Gray, H. 1918. "Anatomy of the Human Body". Philadelphia:Lea \& Febiger.

Groat, B Y William C D E, and William R Saum. 1976. "Synaptic Transmission in Parasynpathetic Ganglia in the Urinary Bladder of the Cat" J. Physiol. 256:137-158.

Groat, W C de. 1995. "Mechanisms Underlying the Recovery of Lower Urinary Tract Function Following Spinal Cord Injury." Paraplegia 33 (9): 493-505. doi:10.1038/sc.1995.109. 
Groat, W C de, and A M Booth. 1980. "Inhibition and Facilitation in Parasympathetic Ganglia of the Urinary Bladder.” Fed Proc 39 (12): 2990-96.

Groat, W C de, and N Yoshimura. 2010. "Changes in Afferent Activity after Spinal Cord Injury.” Neurourol Urodyn 29 (1): 63-76. doi:10.1002/nau.20761.

Groat, W C de, and N Yoshimura. 2012. "Plasticity in Reflex Pathways to the Lower Urinary Tract Following Spinal Cord Injury.” Exp Neurol 235 (1): 123-32.

doi:10.1016/j.expneurol.2011.05.003.

Groat, W C de, and N Yoshimura. 2015. "Anatomy and Physiology of the Lower Urinary Tract." Handb Clin Neurol 130: 61-108. doi:10.1016/B978-0-444-63247-0.00005-5.

Groat, William C. De, Isao Araki, Margaret A. Vizzard, Mitsuharu Yoshiyama, Naoki Yoshimura, Kimio Sugaya, Changfeng Tai, and James R. Roppolo. 1998.

"Developmental and Injury Induced Plasticity in the Micturition Reflex Pathway." Behavioural Brain Research 92 (2): 127-40. doi:10.1016/S0166-4328(97)00185-X.

de Groat, WC; Kawatani ,M. 1989. "Reorganization of sympathetic preganglionic connections in cat bladder ganglia following parasympathetic denervation." $J$ Physiol (Lond) 409:431-449.

de Groat, W.C., Yoshimura, N., 2006." Mechanisms underlying the recovery of lower urinary tract function following spinal cord injury." Prog. Brain Res. 152, 59-84.

Grossman, S D, B B Wolfe, R P Yasuda, and J R Wrathall. 1999. "Alterations in AMPA Receptor Subunit Expression after Experimental Spinal Cord Contusion Injury." The Journal of Neuroscience: The Official Journal of the Society for Neuroscience 19 (14): 5711-20.

Grossman, S D, B B Wolfe, R P Yasuda, and J R Wrathall. 2000. "Changes in NMDA Receptor Subunit Expression in Response to Contusive Spinal Cord Injury." Journal of Neurochemistry 75 (1): 174-84. http://www.ncbi.nlm.nih.gov/pubmed/10854260.

Hill. 2015. Clinical Journal of the American Society of Nephrology10: 480-492. doi: 10.2215/CJN.04520413

Husch A, Van Patten GN, Hong DN, Scaperotti MM, Cramer N, Harris-Warrick RM. 2012." Spinal cord injury induces serotonin supersensitivity without increasing intrinsic excitability of mouse V2a interneurons." Journal of Neuroscience 32:13145-13154.

Inskip, J A, L M Ramer, M S Ramer, and A V Krassioukov. 2009. “Autonomic Assessment of Animals with Spinal Cord Injury: Tools, Techniques and Translation." Spinal Cord 47 (1): 2-35. doi:10.1038/sc.2008.61. 
Jacob, M H. 1991. “Acetylcholine Receptor Expression in Developing Chick Ciliary Ganglion Neurons.” J Neurosci 11 (6): 1701-12.

Jensen, A.A.,Frolund,B.,Liljefors,T.,Krogsgaard-Larsen,P.(2005). Neuronal acetylcholine receptors: Structural revelations, target identifications, and therapeutc inspirations. Journal of Medical Chemistry, 48 (15),4705-4745.

Jobling, P, and R Lim. 2008. "Anatomical and Physiological Properties of Pelvic Ganglion Neurons in Female Mice.” Auton Neurosci 140 (1-2): 30-39. doi:10.1016/j.autneu.2008.03.001.

Kanjhan, R., Osborne, P.B., Ouyang, M., Keast, J.R., 2003. "Postnatal maturational changes in rat pelvic autonomic ganglion cells: a mixture of steroid-dependent and independent effects." Journal of Neurophysiology 89, 315-323

Keast, J R. 1995. "Visualization and Immunohistochemical Characterization of Sympathetic and Parasympathetic Neurons in the Male Rat Major Pelvic Ganglion." Neuroscience 66 (3): 655-62.

Keast, J R, A M Booth, and W C de Groat. 1989. "Distribution of Neurons in the Major Pelvic Ganglion of the Rat Which Supply the Bladder, Colon or Penis." Cell Tissue Res 256 (1): 105-12. http://www.ncbi.nlm.nih.gov/pubmed/2713886.

Keast, J R, and W C de Groat. 1989. "Immunohistochemical Characterization of Pelvic Neurons Which Project to the Bladder, Colon, or Penis in Rats." J Comp Neurol 288 (3): 387-400. doi:10.1002/cne.902880303.

Kepper, M.E., Keast, J.R., 1998. "Specific targeting of ganglion cell sprouts provides an additional mechanism for restoring peripheral motor circuits in pelvic ganglia after spinal nerve damage." J. Neurosci. 18, 7987-7995

Kihara, K, and W C de Groat. 1997. "Sympathetic Efferent Pathways Projecting Bilaterally to the Vas Deferens in the Rat." Anat Rec 248 (2): 291-99. http://www.ncbi.nlm.nih.gov/pubmed/9185995.

Kim, H G, S M Cho, C K Lee, and S W Jeong. 2015. "Neuregulin 1 as an Endogenous Regulator of Nicotinic Acetylcholine Receptors in Adult Major Pelvic Ganglion Neurons.” Biochem Biophys Res Commun 463 (4): 632-37. doi:10.1016/j.bbrc.2015.05.113.

Kim, H G, C K Lee, S M Cho, K Whang, B H Cha, J H Shin, K H Song, and S W Jeong. 2013. "Neuregulin 1 up-Regulates the Expression of Nicotinic Acetylcholine Receptors through the ErbB2/ErbB3-PI3K-MAPK Signaling Cascade in Adult Autonomic Ganglion Neurons." J Neurochem 124 (4): 502-13. doi:10.1111/jnc.12109. 
Kim, S J, D S Lee, W J Bae, S Kim, S H Hong, J Y Lee, T K Hwang, and S W Kim. 2013. "Functional and Molecular Changes of the Bladder in Rats with Crushing Injury of Nerve Bundles from Major Pelvic Ganglion to the Bladder: Role of RhoA/Rho Kinase Pathway." Int J Mol Sci 14 (9): 17511-24. doi:10.3390/ijms140917511.

Klingman, J D. 1969. "Cholinesterases of Rat Sympathetic Ganlgia After Immunosympathectomy, Decentralization and Axotomy". J Neurochem. 16 (2) $: 261-8$

Kottick, A., and C. A. Del Negro. 2015. "Synaptic Depression Influences InspiratoryExpiratory Phase Transition in Dbx 1 Interneurons of the preBotzinger Complex in Neonatal Mice." Journal of Neuroscience 35 (33): 11606-11. doi:10.1523/JNEUROSCI.0351-15.2015.

Krenz, N R, and L C Weaver. 1998. "Changes in the Morphology of Sympathetic Preganglionic Neurons Parallel the Development of Autonomic Dysreflexia after Spinal Cord Injury in Rats." Neuroscience Letters 243 (1-3): 61-64. doi:10.1016/S0304-3940(98)00101-3.

Kruse, M. N., L. A. Bray, and W. C. de Groat. 1995. "Influence of Spinal Cord Injury on the Morphology of Bladder Afferent and Efferent Neurons." Journal of the Autonomic Nervous System 54 (3): 215-24. doi:10.1016/0165-1838(95)00011-L.

Kuntz, A. 1936."The Autonomic Nervous System". The Journal of the American Medical Association 106 (5):345-350.

Langworthy, O.R. (1965). Innervation of the pelvic organs of the rat. Investigative Urology.2(5),491-511.

Levey, Marjory Schwartz, Craig L. Brumwell, Stuart E. Dryer, and Michele H. Jacob. 1995. "Innervation and Target Tissue Interactions Differentially Regulate Acetylcholine Receptor Subunit mRNA Levels in Developing Neurons in Situ." Neuron 14 (1): 153-62. doi:10.1016/0896-6273(95)90249-X.

Li, Y, Philip J. Harvey, X Li, and David J Bennett. 2004. "Spastic Long-Lasting Reflexes of the Chronic Spinal Rat Studied in Vitro." Journal of Neurophysiology 91 (5): 2236-46. doi:10.1152/jn.01010.2003.

Llewellyn-Smith IJ, Weaver LC. 2001. "Changes in synaptic inputs to sympathetic preganglionic neurons after spinal cord injury." J Comp Neurol 435:226-240.

Llewellyn-Smith, I J, L C Weaver, and J R Keast. 2006. "Effects of Spinal Cord Injury on Synaptic Inputs to Sympathetic Preganglionic Neurons." Prog Brain Res 152: 11-26. doi:10.1016/s0079-6123(05)52001-6. 
McCann, C M, J C Tapia, H Kim, J S Coggan, and J W Lichtman. 2008. "Rapid and Modifiable Neurotransmitter Receptor Dynamics at a Neuronal Synapse in Vivo." Nat Neurosci 11 (7): 807-15. doi:10.1038/nn.2145.

Mccorry, Laurie Kelly. 2007. "TEACHERS' TOPICS Physiology of the Autonomic Nervous System" 71 (4).

Miles, R., and R. K. S. Wong. 1986. Excitatory synaptic interactions between CA3 neurones in the guinea-pig hippocampus.J. Physiol.(Lond.) 373: 397-4 18

Milton, Nathaniel G N, Alain Bessis, Jean-Pierre Changeux, and David S Latchman. 1996. "Differential Regulation of Neuronal Nicotinic Acetylcholine Receptor Subunit Gene Promoters by Brn-3 POU Family Transcription Factors.” Biochem. J 317: 419-23.

Murray, Katherine C, Aya Nakae, Marilee J Stephens, Michelle Rank, Jessica D Amico, Philip J Harvey, Xiaole Li, et al. 2010. "Recovery of Motoneuron and Locomotor Function after Spinal Cord Injury Depends on Constitutive Activity in 5-HT2C Receptors" 16 (6): 694-700. doi:10.1038/nm.2160.Recovery.

Nangle, M R, and J R Keast. 2009. "Deafferentation and Axotomy Each Cause Neurturin-Independent Upregulation of c-Jun in Rodent Pelvic Ganglia.” Exp Neurol 215 (2): 271-80. doi:10.1016/j.expneurol.2008.10.012.

Nasiripourdori, A, Taly, V, Grutter, T, and Taly, A. 2011. "From Toxins Targeting Ligand Gated Ion Channels to Therapeutic Molecules." Toxins (3): 260-293. doi:10.3390/toxins3030260

Neher, Erwin, and Takeshi Sakaba. 2008. "Multiple Roles of Calcium Ions in the Regulation of Neurotransmitter Release.” Neuron 59 (6): 861-72. doi:10.1016/j.neuron.2008.08.019.

Okatan, M, Grossberg, S.2000."Frequency-Dependent Synaptic Potentiation, Depression, and Spike Timing Induced by Hebbian Pairing in Cortical Pyramidal Neurons."Neural Networks. Technical Report CAS/CNS-2000-003

Park, K S, S K Cha, M J Kim, D R Kim, S W Jeong, J W Lee, and I D Kong. 2006. “An alpha3beta4 Subunit Combination Acts as a Major Functional Nicotinic Acetylcholine Receptor in Male Rat Pelvic Ganglion Neurons.” Pflugers Arch 452 (6): 775-83. doi:10.1007/s00424-006-0086-1.

Parker, M J, A Beck, and C W Luetje. 1998. "Neuronal Nicotinic Receptor beta2 and beta4 Subunits Confer Large Differences in Agonist Binding Affinity." Molecular Pharmacology 54 (6): 1132-39. http://www.ncbi.nlm.nih.gov/pubmed/9855644. 
Pastelin, C F, R Juarez, M S Damaser, and Y Cruz. 2012. "Neural Pathways of Somatic and Visceral Reflexes of the External Urethral Sphincter in Female Rats." J Comp Neurol 520 (14): 3120-34. doi:10.1002/cne.23079.

Peddie, C J, and J R Keast. 2011. "Pelvic Nerve Injury Causes a Rapid Decrease in Expression of Choline Acetyltransferase and Upregulation of c-Jun and ATF-3 in a Distinct Population of Sacral Preganglionic Neurons." Front Neurosci 5: 6. doi:10.3389/fnins.2011.00006.

Persyn, S, J Gillespie, J Eastham, and S De Wachter. 2016. "Possible Role of the Major Pelvic Ganglion in the Modulation of Non-Voiding Activity in Rats." Auton Neurosci 198: 33-37. doi:10.1016/j.autneu.2016.06.002.

Purinton, P.T.,Fletcher, T.F.,Bradley,W.E.1972. Gross and light microscopic features of the pelvic plexus in the rat. Anat. Rec 175: 697-706.

Purves, B Y Dale. 1975. "Functional and Structural Changes in Mammalian Symmpathetic Neurons Following Interruption of Their Axons," J. Physiol. 252: 429-463.

Rogers H,Henderson G. 1990. "Activation of $\mu$-and $\delta$-opioid receptors present on the same nerve terminals depresses transmitter release in the mouse hypogastric ganglion. Journal of the Autonomic Nervous System. 29 :255-270.

Santin, Joseph M., Mauricio Vallejo, and Lynn K. Hartzler. 2017. "Synaptic up-Scaling Preserves Motor Circuit Output after Chronic, Natural Inactivity." eLife 6. doi:10.7554/eLife.30005.

Sargent PB (1993) "The diversity of neuronal nicotinic acetylcholine receptors." Annu Rev Neurosci 16:403-443

Skok, V. (2002)." Nicotinic acetylcholine receptors in autonomic ganglia." Autonomic Neuroscience: Basic and Clinical, 97, 1-11.

Springer, Mitchell G., Paul H.M. Kullmann, and John P. Horn. 2015. "Virtual Leak Channels Modulate Firing Dynamics and Synaptic Integration in Rat Sympathetic Neurons: Implications for Ganglionic Transmission in Vivo.” Journal of Physiology 593 (4): 803-23. doi:10.1113/jphysiol.2014.284125.

Streichert, L. C., Sargent, P. B. 1992. "The role of acetylcholinesterase in denervation supersensitivity in the frog cardiac ganglion." J. Physiol. 445: 249-60

Sugaya, K.,\& de Groat, W.C.2007."Bladder volume-dependent excitatory and inhibitory influence of lumbosacral dorsal and ventral roots on bladder activity in rats."

Biomed Res. 28(4): 169-175. 
Tabatabai, M.,Booth, A.M.,De Groat, W.1986." Morphological and electrophysiological properties of pelvic ganglion cells in the rat." Brain Research, 382, 61-70.

Takahashi, R, T Yoshizawa, T Yunoki, P Tyagi, S Naito, W C de Groat, and N Yoshimura. 2013. "Hyperexcitability of Bladder Afferent Neurons Associated with Reduction of Kv1.4 Alpha-Subunit in Rats with Spinal Cord Injury." J Urol 190 (6): 2296-2304. doi:10.1016/j.juro.2013.07.058.

Tan, H; G. M. Mawe; and M. A. Vizzard. 2007. "Electrical Properties of Neurons in the Intact Rat Major Pelvic Ganglion.” Auton Neurosci 134 (1-2): 26-37.

doi:10.1016/j.autneu.2007.01.013.

Thesleff, S., \& Sellin , L.C.1980. "Denervation supersensitivity". Trends in Neuroscience 3(5): 122-126.

Tuttle, JB; Steers, WD; Albo, M; Nataluk, E. 1994. "Neural input regulates tissue NGF and growth of the adult rat urinary bladder." J Auton Nerv Syst 49:147-158

Vizzard, Margaret A. 2006. "Neurochemical Plasticity and the Role of Neurotrophic Factors in Bladder Reflex Pathways after Spinal Cord Injury” 152: 97-115. doi:10.1016/s0079-6123(05)52007-7.

Wang, N, A Orr-Urtreger, J Chapman, R Rabinowitz, R Nachman, and A D Korczyn. 2002. "Autonomic Function in Mice Lacking alpha5 Neuronal Nicotinic Acetylcholine Receptor Subunit.” J Physiol 542 (Pt 2): 347-54. doi:10.1113/jphysiol.2001.013456.

Wang, Ningshan, Avi Orr-Urtreger, Joab Chapman, Ruth Rabinowitz, Rachel Nachman, and Amos D. Korczyn. 2002. "Autonomic Function in Mice Lacking a5 Neuronal Nicotinic Acetylcholine Receptor Subunit.” The Journal of Physiology 542 (2): 347-54. doi:10.1113/jphysiol.2001.013456.

Wang, X. 2005. “Activity-Dependent Presynaptic Regulation of Quantal Size at the Mammalian Neuromuscular Junction In Vivo." Journal of Neuroscience 25 (2): 343-51. doi:10.1523/JNEUROSCI.3252-04.2005.

Wanigasekara, Y, M E Kepper, and J R Keast. 2003. "Immunohistochemical Characterisation of Pelvic Autonomic Ganglia in Male Mice." Cell Tissue Res 311 (2): 175-85. doi:10.1007/s00441-002-0673-1.

Won, Y J, K Whang, I D Kong, K S Park, J W Lee, and S W Jeong. 2006. "Expression Profiles of High Voltage-Activated Calcium Channels in Sympathetic and Parasympathetic Pelvic Ganglion Neurons Innervating the Urogenital System." J Pharmacol Exp Ther 317 (3): 1064-71. doi:10.1124/jpet.105.098210. 
Wonnacott, S., Barik, J. 2007. Nicotinic ACh Receptors. Tocris Bioscience Scientific Review Series, 1-19.

$\mathrm{Xu}$, Jianhua, and Ling Gang Wu. 2005. "The Decrease in the Presynaptic Calcium Current Is a Major Cause of Short-Term Depression at a Calyx-Type Synapse." Neuron 46 (4): 633-45. doi:10.1016/j.neuron.2005.03.024.

Xu, W, S Gelber, A Orr-Urtreger, D Armstrong, R a Lewis, C N Ou, J Patrick, L Role, M De Biasi, and a L Beaudet. 1999. "Megacystis, Mydriasis, and Ion Channel Defect in Mice Lacking the alpha3 Neuronal Nicotinic Acetylcholine Receptor."

Proceedings of the National Academy of Sciences of the United States of America 96 (10): 5746-51. doi:10.1073/pnas.96.10.5746.

Xu, W, A Orr-Urtreger, F Nigro, S Gelber, C B Sutcliffe, D Armstrong, J W Patrick, L W Role, A L Beaudet, and M De Biasi. 1999. "Multiorgan Autonomic Dysfunction in Mice Lacking the beta2 and the beta4 Subunits of Neuronal Nicotinic Acetylcholine Receptors." The Journal of Neuroscience : The Official Journal of the Society for Neuroscience 19 (21): 9298-9305. http://www.ncbi.nlm.nih.gov/pubmed/10531434.

Yeh, J, M Ferreira, S Ebert, R P Yasuda, K J Kellar, and B B Wolfe. 2001. “Axotomy and Nerve Growth Factor Regulate Levels of Neuronal Nicotinic Acetylcholine Receptor alpha3 Subunit Protein in the Rat Superior Cervical Ganglion." $J$ Neurochem 79 (2): 258-65.

Yoshimura, N., \& De Groat,W.C. 1996. "Characterization of Voltage-Sensitive Na+ and K+ Currents Recorded from Acutely Dissociated Pelvic Ganglion Neurons of the Adult Rat." J Neurophysiol 76 (4): 2508-21.

Yoshimura, N., \& De Groat, W.C. (1997). "Neural control of the lower urinary tract." Int J Urol (4): 111-125.

Yoshimura, N.,\& De Groat, W. C.1997. "Plasticity of $\mathrm{Na}^{+}$Channels in Afferent Neurones Innervating Rat Urinary Bladder Following Spinal Cord Injury." Journal of Physiology 503 (2): 269-276.

Yoshimura, N., (1999). "Bladder afferent pathway and spinal cord injury: Possible mechanisms inducing hyperreflexia of the urinary bladder." Progress in Neurobiology 57:583-606.

Young, T, S Wittenauer, R Parker, and M Vincler. 2008. "Peripheral Nerve Injury Alters Spinal Nicotinic Acetylcholine Receptor Pharmacology." Eur J Pharmacol 590 (13): $163-69$.

Zhou, Yuefang, Evan Deneris, and Richard E. Zigmond. 1998. "Differential Regulation of Levels of Nicotinic Receptor Subunit Transcripts in Adult Sympathetic Neurons after Axotomy." Journal of Neurobiology 34 (2): 164-78. 


\section{VITA}

I was born and raised in Yangon, Myanmar. I came to the United Stated to attend Concordia College in Moorhead, Minnesota, in 2008. It was during my time at Concordia that my fascination with Neuroscience began. As I was taking classes such as Introduction to Neuroscience, Cognitive Psychology, and Behavioral Neuroscience, I was intrigued by the ability of our brain to learn, store, and remember information throughout our lifetime. I was also surprised by the 'plasticity' of the nervous system after injury and neurological diseases.

Beginning in the summer after junior year of college, I began to get involved in research as a student volunteer and worked on a project that studied cognitive effects of systemic lupus erythematosus.

After graduating from college in 2012, I worked as a research assistant at the University of California, San Diego. Then, in 2013, I began my graduate career in Division of Biological Sciences at the University of Missouri, Columbia. I rotated in Dr. McClellan's lab for the first semester before joining the laboratory of Dr. David Schulz where I carried out research for my dissertation on "plasticity of the neurons in mouse major pelvic ganglia in response to loss of physiological input in cases of nerve injury and spinal cord injury'.

I plan to pursue a career as a medical writer and plan to stay involved in science throughout my career. I have accepted a position as health communications intern at National Cancer Institute (NCI), NIH. 